\title{
PÄR JOHANSSON ON HYDROXYAPATITE MODIFIED PEEK IMPLANTS FOR BONE APPLICATIONS
}
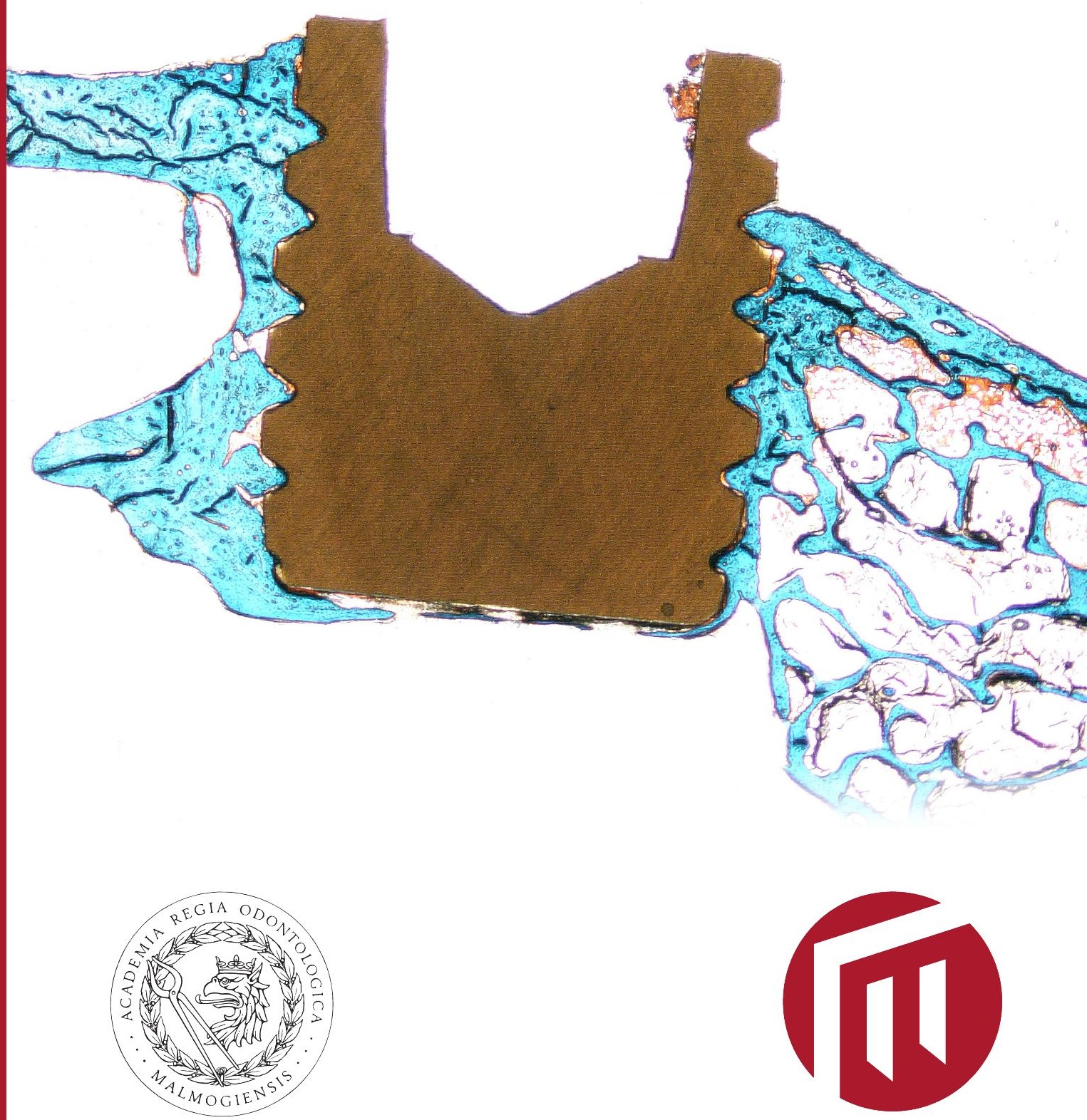

ON HYDROXYAPATITE MODIFIED PEEK IMPLANTS

FOR BONE APPLICATIONS 
Malmö University

Faculty of Odontology Doctoral Dissertation 2017

(C) Pär Johansson 2017

ISBN 978-9I-7 IO4-80I-I (print)

ISBN 978-9I-7IO4-802-8 (pdf)

Holmbergs, Malmö 2017 


\title{
PÅR JOHANSSON ON HYDROXYAPATITE MODIFIED PEEK IMPLANTS FOR BONE APPLICATIONS
}

\author{
Malmö University, 2017 \\ Faculty of Odontology \\ Department of Prosthodontics
}


Publikationen finns även elektroniskt, se www.mah.se/muep 
I dedicated this work to my best friend and partner in life, Kristin, for your unconditional love and support. I love you so much 

This thesis is number 51 in a series of investigations on implants, hard tissues and the locomotor apparatus originating from the Department of Biomaterials, University of Gothenburg and the Department of Prosthodontics/Material Sciences, Malmö University, Sweden.

1. Anders R Eriksson DDS, 1984. Heat-induced Bone Tissue Injury. An in vivo investigation of heat tolerance of bone tissue and temperature rise in the drilling of cortical bone.

Thesis defended 21.2.1984. External examiner: Docent K-G. Thorngren.

2. Magnus Jacobsson MD, 1985. On Bone Behaviour after Irradiation.

Thesis defended 29.4.1985. External examiner: Docent A. Nathanson.

3. Fredric Buch MD, 1985. On Electrical Stimulation of Bone Tissue.

Thesis defended 28.5.1985. External examiner: Docent T. Ejsing-Jörgensen.

4. Peter Kälebo MD, 1987. On Experimental Bone Regeneration in Titanium Implants. A quantitative microradiographic and histologic investigation using the Bone Harvest Chamber. Thesis defended 1.10.1987. External examiner: Docent N. Egund.

5. Lars Carlsson MD, 1989. On the Development of a new Concept for Orthopaedic Implant Fixation.

Thesis defended 2.12.1989. External examiner: Docent L- $\AA$ Broström.

6. Tord Röstlund MD, 1990. On the Development of a New Arthroplasty.

Thesis defended 19.1.1990. External examiner: Docent Å. Carlsson.

7. Carina Johansson Res Tech, 1991. On Tissue Reaction to Metal Implants.

Thesis defended 12.4.1991. External examiner: Professor K. Nilner.

8. Lars Sennerby DDS, 1991. On the Bone Tissue Response to Titanium Implants. 
Thesis defended 24.9.1991. External examiner: Dr J.E. Davies.

9. Per Morberg MD, 1991. On Bone Tissue Reactions to Acrylic Cement.

Thesis defended 19.12.1991. External examiner: Docent K. Obrant.

10. Ulla Myhr PT, 1994. On factors of Importance for Sitting in Children with Cerebral Palsy.

Thesis defended 15.4.1994. External examiner: Docent K. Harms-Ringdahl.

11. Magnus Gottlander MD, 1994. On Hard Tissue Reactions to Hydroxyapatite- Coated Titanium Implants.

Thesis defended 25.11.1994. External examiner: Docent P. Aspenberg.

12. Edward Ebramzadeh MScEng, 1995. On Factors Affecting Long-Term Outcome of Total Hip Replacements.

Thesis defended 6.2.1995. External examiner: Docent L. Linder.

13. Patricia Campbell BA, 1995. On Aseptic Loosening in Total Hip Replacement: the Role of UHMWPE Wear Particles.

Thesis defended 7.2.1995. External examiner: Professor D. Howie.

14. Ann Wennerberg, DDS, 1996. On Surface Roughness and Implant Incorporation.

Thesis defended 19.4.1996. External examiner: Professor PO. Glantz.

15. Neil Meredith BDS MSc FDS RCSm, 1997. On the Clinical Measurement of Implant Stability Osseointegration.

Thesis defended 3.6.1997. External examiner: Professor J. Brunski.

16. Lars Rasmusson DDS, 1998. On Implant Integration in Membrane-Induced and Grafter Bone.

Thesis defended 4.12.1998. External examiner: Professor R. Haanaes.

17. Thay Q Lee MSc, 1999. On the Biomechanics of the Patellfemoral Joint and Patellar Resurfacing in Total Knee Arthroplasty. 
Thesis defended 19.4.1999. External examiner: Docent G. Nemeth.

18. Anna Karin Lundgren DDS, 1999. On Factors Influencing Guided Regeneration and Augmentation of Intramembraneous Bone.

Thesis defended 7.5.1999. External examiner: Professor B. Klinge.

19. Carl-Johan Ivanoff DDS, 1999. On Surgical and Implant Related Factors Influencing Integration and Function of Titanium Implants. Experimental and Clinical Aspects.

Thesis defended 12.5.1999. External examiner: Professor B. Rosenquist.

20. Bertil Friberg DDS MDS, 1999. On Bone Quality and Implant Stability Measurements.

Thesis defended 12.11.1999. External examiner: Docent P. Åstrand.

21. Åse Allansdotter Johansson $\mathrm{MD}, 1999$. On Implant Integration in Irradiated Bone. An Experimental Study of the Effects of Hyperbaric Oxygeneration and Delayed Implant Placement.

Thesis defended 8.12.1999. External examiner: Docent K. Arvidsson-Fyrberg.

22. Börje Svensson FFS, 2000. On Costochondral Grafts Replacing Mandibular Condyles in Juvenile Chronic Arthritis. A Clinical, Histologic and Experimental Study.

Thesis defended 22.5.2000. External examiner: Professor Ch. Lindqvist.

23. Warren Macdonald BEng, MPhil, 2000. On Component Integration on Total Hip Arthroplasties: Pre-Clinical Evaluations. Thesis defended 1.9.2000. External examiner: Dr A.J.C. Lee.

24. Magne Røkkum MD, 2001. On Late Complications with HA Coated Hip Arthroplasties.

Thesis defended 12.10.2001. External examiner: Professor P. Benum.

25. Carin Hallgren Höstner DDS, 2001. On the Bone Response to Different Implant Textures. A 3D analysis of roughness, wavelength and surface pattern of experimental implants. 
Thesis defended 19.11.2001. External examiner: Professor S. Lundgren.

26. Young-Taeg Sul DDS, 2002. On the Bone Response to Oxidised Titanium Implants: The role of microporous structure and chemical composition of the surface oxide in enhanced osseointegration.

Thesis defended 7.6.2002. External examiner: Professor J.E. Ellingsen.

27. Victoria Franke Stenport DDS, 2002. On Growth Factors and Titanium Implant Integration in Bone.

Thesis defended 11.6.2002. External examiner: Associate Professor E. Solheim.

28. Mikael Sundfeldt MD, 2002. On the Aetiology of Aseptic Loosening in Joint Arthroplasties and Routes to Improved cemented Fixation.

Thesis defended 14.6.2002. External examiner: Professor N. Dahlén.

29. Christer Slotte CCS, 2003. On Surgical Techniques to Increase Bone Density and Volume. Studies in Rat and Rabbit. Thesis defended 13.6.2003. External examiner: Professor C.H.F. Hämmerle.

30. Anna Arvidsson MSc, 2003. On Surface Mediated Interactions Related to Chemomechanical Caries Removal. Effects on surrounding tissues and materials.

Thesis defended 28.11.2003. External examiner: Professor P. Tengvall.

31. Pia Bolind DDS, 2004. On 606 retrieved oral and craniofacial implants. An analysis of consequently received human specimens.

Thesis defended 17.12.2004. External examiner: Professor A. Piattelli.

32. Patricia Miranda Burgos DDS, 2006. On the influence of micro- and macroscopic surface modifications on bone integration of titanium implants.

Thesis defended 1.9.2006. External examiner: Professor A. Piattelli. 
33. Jonas P. Becktor DDS, 2006. On factors influencing the outcome of various techniques using endosseous implants for reconstruction of the atrophic edentulous and partially dentate maxilla.

Thesis defended 17.11.2006. External examiner: Professor K.F. Moos.

34. Anna Göransson DDS, 2006. On Possibly Bioactive CP Titanium Surfaces.

Thesis defended 8.12.2006. External examiner: Professor B. Melsen.

35. Andreas Thor DDS, 2006. On platelet-rich plasma in reconstructive dental implant surgery.

Thesis defended 8.12.2006. External examiner: Professor E.M. Pinholt.

36. Luiz Meirelles DDS MSc, 2007. On Nano Size Structures for Enhanced Early Bone Formation.

Thesis defended 13.6.2007. External examiner: Professor Lyndon F. Cooper.

37. Pär-Olov Östman DDS, 2007. On various protocols for direct loading of implant-supported fixed prostheses.

Thesis defended 21.12.2007. External examiner: Professor B. Klinge.

38. Kerstin Fischer DDS, 2008. On immediate/early loading of implant supported prostheses in the maxilla.

Thesis defended 8.2.2008. External examiner: Professor K. Arvidsson Fyrberg.

39. Alf Eliasson 2008. On the role of number of fixtures, surgical technique and timing of loading.

Thesis defended 23.5.2008. External examiner: Professor K. Arvidsson Fyrberg.

40. Victoria Fröjd DDS, 2010. On Ca2+ incorporation and nanoporosity of titanium surfaces and the effect on implant performance.

Thesis defended 26.11.2010. External examiner: Professor J.E. Ellingsen.

41. Lory Melin Svanborg DDS, 2011. On the importance of nanometer structures for implant incorporation in bone tissue. 
Thesis defended 01.06.2011. External examiner: Associate professor C. Dahlin.

42. Byung-Soo Kang MSc, 2011. On the bone tissue response to surface chemistry modifications of titanium implants.

Thesis defended 30.09.2011. External examiner: Professor J. Pan.

43. Kostas Bougas DDS, 2012. On the influence of biochemical coating on implant bone incorporation.

Thesis defended 12.12.2012. External examiner: Professor T. Berglundh.

44. Arne Mordenfeld DDS, 2013. On tissue reaction to and adsorption of bone substitutes.

Thesis defended 29.5.2013. External examiner: Professor C. Dahlin.

45. Ramesh Chowdhary DDS, 2014. On efficacy of implant thread design for bone stimulation.

Thesis defended 21.05.2014. External examiner: Professor Flemming Isidor.

46. Anders Halldin MSc, 2015. On a biomechanical approach to analysis of stability and load bearing capacity of oral implants.

Thesis defended 28.05.2015. External examiner: Professor J. Brunski.

47. Francesca Cecchinato MSc, 2015. On magnesium-modified titanium coatings and magnesium alloys for oral and orthopaedic applications: in vitro investigation.

Thesis defended 20.11.2015. External examiner: Professor C. Stanford.

48. Jonas Anderud DDS, 2016. On guided bone regeneration using ceramic membranes.

Thesis defended 27.05.2016. External examiner: Professor S. Lundgren

49. Silvia Galli DDS, 2016. On magnesium-containing implants for bone applications.

Thesis defended 08.12.2016. External examiner: Professor J.E. Ellingsen.

50. Bruno Chrcanovic DDS MSc, 2017. On Failure of Oral Implants. 
Thesis defended 08.06.2017. External examiner: Associate Professor B. Friberg.

51. Pär Johansson DDS, 2017. On hydroxyapatite modified PEEK implants for bone applications.

Thesis to be defended 15.12.2017. External examiner: Professor L. Rasmusson.

see www.mah.se/muep 



\section{TABLE OF CONTENTS}

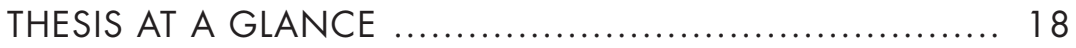

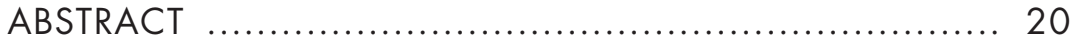

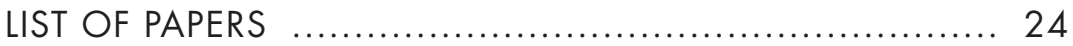

Contribution by the respondent ….................................... 25

ABBREVIATIONS, ACRONYMS AND SYMBOLS ............. 26

PEEK AS A BIOMATERIAL .................................. 30

Definitions in biomaterial science ...................................... 30

Basic principles of Polymers ............................................ 31

Polymers in the Medical Field ........................................... 33

Properties of PEEK Polymers .............................................. 34

Processing and Thermal Transitions ............................... 35

Mechanical properties .............................................. 37

Chemical and radiation stability ................................. 40

Clinical applications .................................................. 41

Biocompatibility and bioactive modifications of PEEK ............ 50

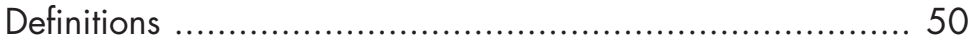

Cytotoxicity and genotoxicity of bulk PEEK ..................... 50

Animal studies of bulk PEEK ......................................... 51

Bioactive modifications of PEEK ................................... 52

AIMS ......................................................... 64

MATERIALS AND METHODS ................................ 65

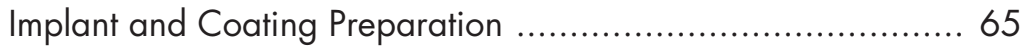

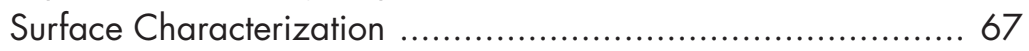

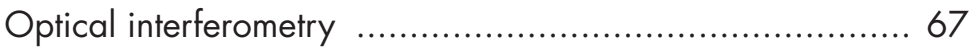

Atomic force microscopy .............................................. 68

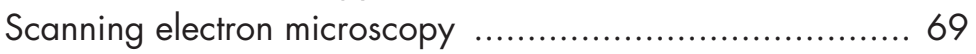

Contact angle measurements ...................................... 70 
Chemical and Mechanical Characterization ......................... 71

X-ray photon spectroscopy .......................................... 71

Coating adhesion in Sawbones $₫$ simulation model ........... 71

Tensile testing ............................................................ 72

Experimental Animal Model ....................................... 72

Animals and ethical approvals .................................... 72

Anesthesia ......................................................... 73

Surgical procedure and implantation ............................. 73

Experimental model .................................................. 74

Post-operative care and euthanasia ............................... 75

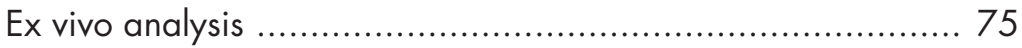

Removal torque testing ….......................................... 75

Gene expression analysis ........................................ 76

Bone histomorphometry ....................................................... 79

X-ray computed microtomography ............................... 81

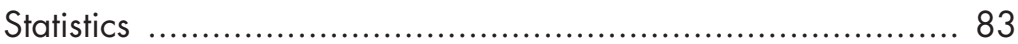

SUMMARY OF RESULTS …................................ 85

Surface Characterization ................................................... 85

Chemical and Mechanical Characterization ........................ 87

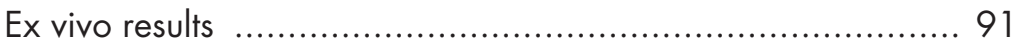

Removal torque results ............................................ 92

Gene expression results ............................................... 94

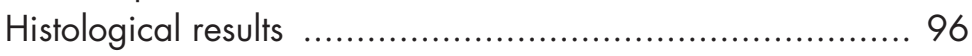

Micro-CT results ..................................................... 105

DISCUSSION ................................................ 109

Structural and chemical properties of the HA-coating ........... 109

Biomechanical properties of HA coated implants ................ 117

Bone formation around PEEK and HA-PEEK ....................... 119

Genetic mechanisms ........................................... 127

CONCLUSION AND FUTURE PERSPECTIVES ............... 131

POPULÄRVETENSKAPLIG SAMMANFATTNING ............ 134

ACKNOWLEDGMENTS ................................... 136

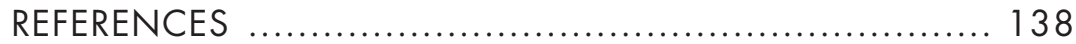

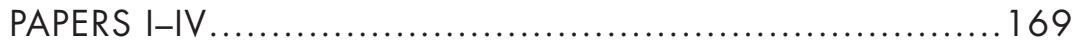





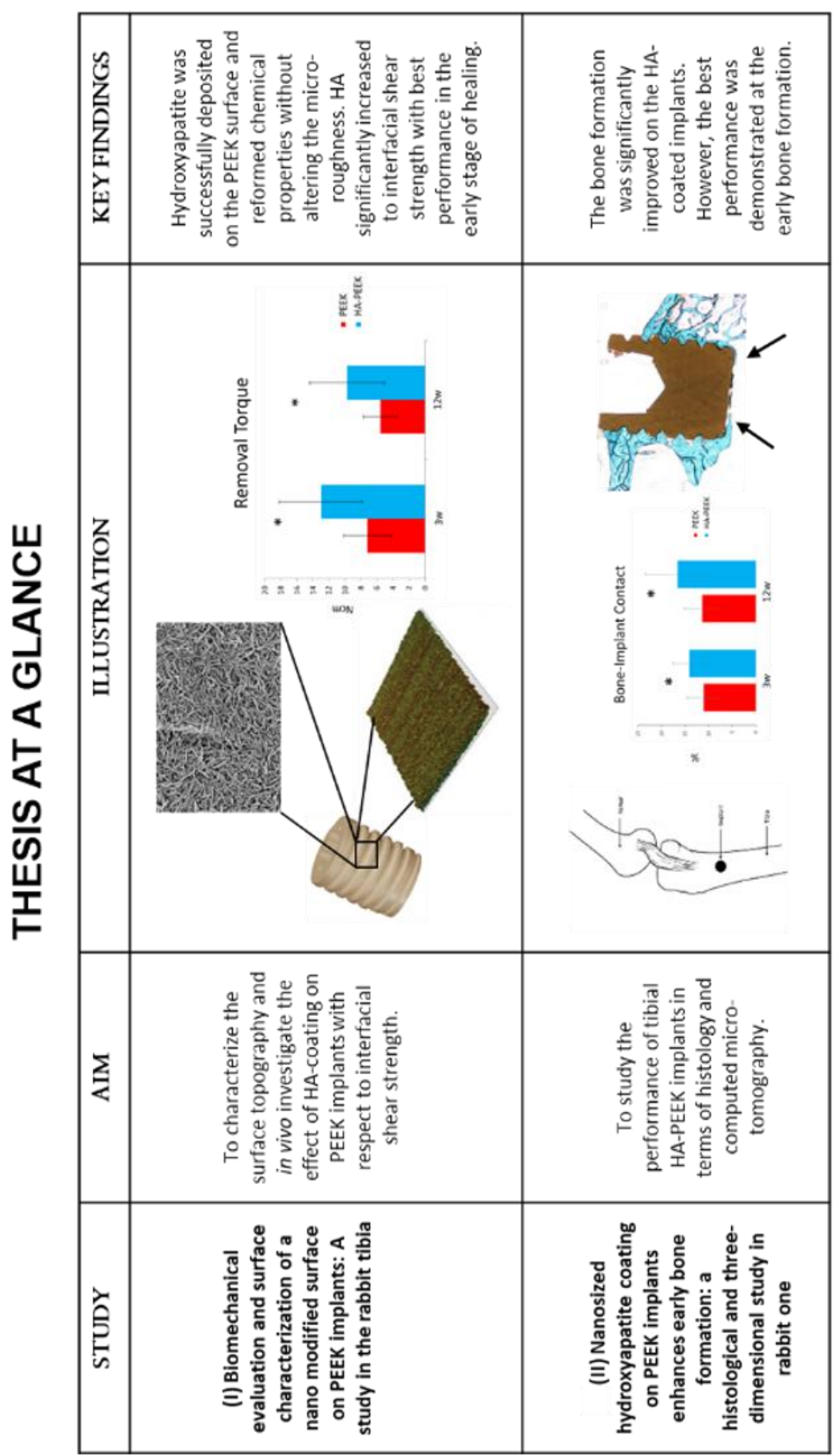




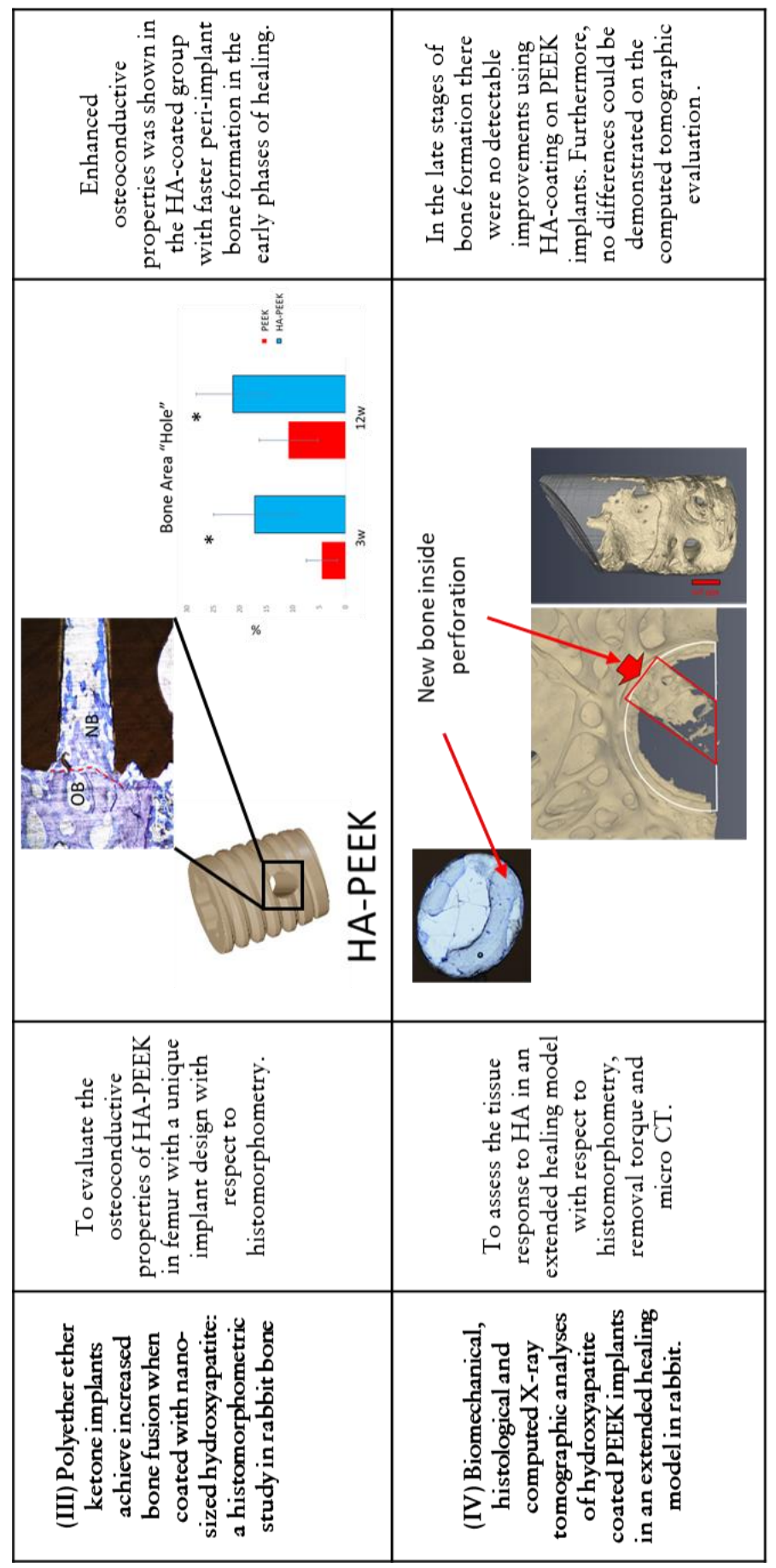




\section{ABSTRACT}

People are living longer today than ever before, and the number of older adults will increase exponentially over the next coming years. However, that does not necessarily mean that they are living healthier. An aging population presents many opportunities but also challenges in the public health care with a sharp increase in incidence of many diseases associated to the bone, teeth and joint systems. Orthopedic and dental implants commonly used mostly consist of metals, composites, polymers and ceramics.

Titanium has been widely used in the orthopedic and dental field to replace or support mineralized structures. Titanium possesses good mechanical, physical and biologic properties. However, titanium has in the clinic presented some critical drawbacks. The modulus of elasticity is higher than that of bone which may cause stress shielding and bone resorption ultimately jeopardizing the implant retention. In addition, with rare cases of titanium allergy and patient desires for non-metallic treatments, alternatives to titanium are being studied. Furthermore, the radiopacity of titanium causes interference with the radiographic evaluation and the non-esthetic color may discolor the gingiva of thin biotypes.

Recently, advanced polymeric implantable materials have been introduced into the medical field. Polyetherether ketone (PEEK) is widely used in various orthopedic applications, such as spine implants, joint replacements and fracture fixations. In dentistry, PEEK can be manufactured as prosthetic components, fixed and removable bridges and dentures. 
PEEK is a thermoplastic polymer with mechanical properties that are close to those of human bone, for example the elastic modulus. This will minimize the stress shielding upon loading and stress distribution. Besides, PEEK holds excellent biocompatibility with no toxic or mutagenic effects in vitro and in vivo.

However, unmodified PEEK is biologically inert with limited protein and cell adhesion to the surface. Therefore, improving the bioactivity of PEEK is a significant challenge and a prerequisite to possess all of its potentials as a biomaterial. There are currently 2 methods to improve the bioactivity of PEEK; surface modification and composite preparation. Hydroxyapatite (HA) is the most widely used material due to its biocompatibility and osteoconductive potential in association with biomaterials. There are many developed and evaluated methods to deposit HA on metallic implants, but the only commercially used one is plasma spraying. However, the technique has some severe disadvantages such as irregular coating thickness with deficient adherence to the implant surface. Clinical complications have been confirmed with inflammation caused by fragments of delaminated coating. To challenge these disadvantages, several alternative coating techniques have been introduced.

The aim of this thesis was to evaluate a novel surface modification of bioactive hydroxyapatite deposited by a spin-coating technique. The technique generates a crystalline, nanosized coating homogenously distributed over the implant surface. In particular, the thesis encompasses a comprehensive topographic analysis to evaluate the coating properties. In addition, the HA-PEEK implant will be compared in vivo with an unmodified control with respect to its osteoconductive and bioactive properties.

In study I, the surface topography was characterized using interferometry (IFM), scanning electron microscopy (SEM) and atomic force microscopy (AFM) of the same types of implants that were evaluated in the experimental animal models. The chemical characterization before and after the coating procedure was evaluated with X-ray photon spectroscopy (XPS). Mechanical testing of implants were completed in accordance with the tensile testing standard (ISO 527-2). Furthermore, the hydrophilic appearance after coating was 
estimated by measuring the contact angle. The results from the material characterization reveal a hydrophilic coating with a minimally rough surface which preserved its mechanical properties after the coating procedure. The chemical analysis revealed that only $\mathrm{Ca}, \mathrm{P}$, $\mathrm{C}$ and $\mathrm{O}$ were present at the surface of HA-PEEK with a proportion as that of human bone.

In study I and IV, PEEK implants were implanted in the rabbit tibia and femur and retrieved after 3,12 and 20 weeks of submerged healing and subjected to biomechanical analysis using a removal torque device. The removal torque required to unscrew the implants was significantly higher for HA-PEEK after the 3 and 12 week retrieval time points. However, the absolute torque values were lower at 12 weeks compared to earlier time points, presumably caused by absence of primary stability and dissolution of the HA-coating. After 20 weeks of healing the absolute values were even lower than for the implants recovered at 3 and 12 weeks. However, the retention was still significantly increased for the HA-PEEK implants.

In study II, III and IV, the osseointegration was histologically evaluated with respect to bone-implant contact (BIC) and bone area (BA) after the same healing time points as aforementioned. The performance of the HA-coating was most significant 3 weeks after implantation. However, after 12 and 20 weeks, the BIC turned out to be comparable between the groups. Furthermore, the implants implanted in femur were designed with an apical perforation to evaluate the osteoconductivity in terms of area of bone. After 3 and 12 weeks the results revealed a significant improvement on the HAPEEK. However, after an extended healing period of 20 weeks, the unmodified implants were presenting comparable results as the HAPEEK group.

A more comprehensive description of the osseointegration can be achieved using computed micro-tomography. Three different diameters around the implant and inside the apical perforation were evaluated with respect to bone density and trabecular properties. However, there were no detectable differences between the PEEK and HA-PEEK. The outcome was limited to the resolution of X-rays. There was a minor contrast between resin and PEEK. 
The implant surface, particularly the thread edges are subjected to mechanical forces during implant placement and removal. The stability of the coating was evaluated in study II. The coating was wellpreserved in deformities of the surface, but on the flat edges of the threads there were signs of deformation and absence of HA crystals.

This thesis demonstrated that a nanosized HA-coating can be processed using a spin-coating technique with nanostructures and sufficient adhesion to the substrate.

Furthermore, the performance of the HA-coating was found to improve the surface osteoconductivity by increasing the level and speed of osseointegration. However, the effect was most prominent in the early stages of healing whereas the implants from the extended time points showed osteoconductive properties comparable to unmodified PEEK.

In addition, the ability of HA-coating to improve bone fusion inside a perforation was the most clinical relevant outcome. Unmodified PEEK implants are today widely used in spine surgery and the present surface modifications may improve the clinical outcomes where a rapid bone formation is essential. 


\section{LIST OF PAPERS}

This dissertation is based on the following papers, which will be referred to in the main text by their Roman numerals. The papers are appended at the end of the thesis.

I. Biomechanical evaluation and surface characterization of a nano-modified surface on PEEK implants: A study in the rabbit tibia.

Johansson P, Jimbo R, Kjellin P, Currie F, Chrcanovic B, Wennerberg A.

International Journal of Nanomedicine 2013 Aug 14;9: 390311; doi: 10.2147/IJN.S60387.

II. Nanosized Hydroxyapatite Coating on PEEK Implants Enhances Early Bone Formation: A Histological and Three-Dimensional Investigation in Rabbit Bone.

Johansson P, Jimbo R, Kozai Y, Sakurai T, Kjellin P, Currie F, Wennerberg A.

Materials 2015 Jun 25;8(7):3815-3830; doi: 10.3390/ma8073815.

III: Polyether ether ketone implants achieve increased bone fusion when coated with nano-sized hydroxyapatite: a histomorphometric study in rabbit bone.

Johansson P, Jimbo R, Naito Y, Kjellin P, Currie F, Wennerberg A.

International Journal of Nanomedicine 2016 Apr 6;11: 1435 42; doi: 10.2147/IJN.S100424

IV: Biomechanical, histological and computed X-ray tomographic analyses of hydroxyapatite coated PEEK implants in an extended healing model in rabbit. 
Johansson P, Barkarmo S, Hawthan M, Peruzzi N, Kjellin P, Wennerberg A.

Submitted to Journal of Biomedical Materials Research: Part A

Reprint permissions have been granted from:

Paper I and III: Dove Medical Press Limited, AUT171619

Paper II: MDPI, Open access license (CC, BY)

\section{Contribution by the respondent}

The respondent performed most of the work from planning, experimental work (with exception of the SEM imaging, EDX analysis, contact angle analysis and computed microtomography imaging) and analysis of the data. The respondent was also the main contributor to writing the manuscripts. 


\section{ABBREVIATIONS, ACRONYMS AND SYMBOLS}

\section{Abbreviations}

${ }^{\circ} \mathrm{C}$

$\mu \mathrm{m}$

3D

ACTB

AFM

ALIF

ALP

ANAB

ASD

BA

BGLAP

BIC

BMP2

$\mathrm{BS} / \mathrm{BV}$

$\mathrm{BV} / \mathrm{TV}$

$\mathrm{CaCl}^{2}$

CAD/CAM

CCD

cDNA

CFR-PEEK

$\mathrm{CoCr}$

CoCrMo

COL1A1

CS

$\mathrm{CT}$

DICOM

DNA
Degree Celsius

Micrometer

Three-dimensional

Beta-actin

Atomic force microscopy

Anterior lumbar interbody fusion

Alkaline phosphatase

Accelerated neutral atom beam

Adjacent segment degeneration

Bone area

Bone gamma-carboxyglutamate (GLA) protein

Bone-implant contact

Bone morphogenetic protein-2 Bone surface per given bone volume Bone volume to total volume

Calcium chloride Computer aided design/computer aided manufacturing

Charge coupled device Complementary deoxyribonucleic acid Carbon-fiber-reinforced polyetheretherketone

Cobalt chromium alloy Cobalt chromium molybdenum Collagen type I alpha 1 Calcium silicate Computed tomography Digital Imaging and Communications in Medicine Deoxyribonucleic acid 
EDX

FDA

FDP

FHA

GAPDH

$\mathrm{GPa}$

$\mathrm{h}$

$\mathrm{H} 2 \mathrm{SO} 4$

$\mathrm{HA}$

HA-PEEK

IFM

IGF1

ISO

$\mathrm{kg}$

$\mathrm{kV}$

L-929

LDHA

Ltd

MC3T3-E1

MG-63

micro-CT

$\min$

$\mathrm{mL}$

$\mathrm{mm}$

$\mathrm{MPa}$

$\mathrm{NaOH}$

$\mathrm{Ncm}$

$\mathrm{nm}$

$\mathrm{O}_{2}$

PCR

PDMS

PE

PEEK

$\mathrm{pH}$

PLIF

PMMA

$\mathrm{PP}$

PVC
Energy-dispersice X-ray spectroscopy

Food and Drug Administration

Fixed dental prostheses

Fluorohydroxyapatite

Glyceraldehyde-3-Phosphate Dehydrogenase

Gigapascal

Hours

Sulphuric acid Hydroxyapatite

Hydroxyapatite- polyetheretherketone

Interferometer

Insulin like growth factor 1

International Organization for Standardization

Kilogram

Kilovolt

Mouse fibroblast cells

Lactate Dehydrogenase A

Limited liability company

Mouse osteblastic cell line

Human Osteosarcoma cell Micro-Computed Tomography

Minute

Milliliter

Millimeter

Megapascal

Sodium hydroxide

Newton centimeter

Nanometer

Oxygen gas

Polymerase chain reaction

Polydimethylsiloxane

Polyethylene

Polyetheretherketone

Potential of hydrogen

Posterior lumbar interbody fusion

Poly methyl methacrylate

Polypropylene

Polyvinyl chloride 
qPCR

$\mathrm{R}_{\mathrm{a}}$

RCT

RFA

RNA

RNase

rpm

RTQ

RUNX2

S

SBF

SD

$\mathrm{S}_{\mathrm{a}}$

$\mathrm{S}_{\mathrm{dr}}$

$\mathrm{S}_{\mathrm{ds}}$

SEM

SSP1

Tb.N

Tb.Th

TCP

TCPS

TEM

Th.Sp

$\mathrm{TiO}^{2}$

TJA

TLIF

UHMWPE

USD

W

w/w

VOI

XPS

YSZ

$\AA$

$\beta$-TCP

$\mu \mathrm{A}$

$\mu \mathrm{CT}$

$\mu l$
Quantitative polymerase chain reacton

Arithmetic average of the rougnhess Randomized controlled trial

Radiofrequency ablation

Ribonucleic acid

Ribonuclease

Revolutions per minute

Removal torque

Runt-related transcription factor 2

Second

Simulated body fluid

Standard deviation

Topographical parameter - mean height deviation

Topographical parameter - developed area ratio

Topographical parameter - density of summits

Scanning electron microscopy

Secreted phosphoprotein

Trabecular number

Trabecular thickness

Tissue Culture Plate

Tissue culture polystyrene

Transmission electron microscopy

Trabecular spacing

Titanium dioxide

Total joint arthroplasties

Transforaminal Lumbar Interbody Fusion

Ultra-high-molecular-weight polyethylene

United States Dollar

Wolfram

Weight by weight

Volume of interest

X-ray photoelectron spectroscopy

Yttria-stabilized Zirconia

Ångström

Beta-tricalcium phosphate

Microampere

Micro-computed tomography

Microlitre 
$\mu \mathrm{m}$

Micrometer

Element symbols

$\begin{array}{lr}\mathrm{Al} & \text { Aluminum } \\ \mathrm{Ar} & \text { Argon } \\ \mathrm{C} & \text { Carbon } \\ \mathrm{Ca} & \text { Calcium } \\ \mathrm{Cl} & \text { Chlorine } \\ \mathrm{Co} & \text { Cobalt } \\ \mathrm{Cr} & \text { Chromium } \\ \mathrm{F} & \text { Fluorine } \\ \mathrm{H} & \text { Hydrogen } \\ \mathrm{He} & \text { Helium } \\ \mathrm{Mg} & \text { Magnesium } \\ \mathrm{Mo} & \text { Molybdenum } \\ \mathrm{N} & \text { Nitrogen } \\ \mathrm{Nb} & \text { Niobium } \\ \mathrm{O} & \text { Oxygen } \\ \mathrm{P} & \text { Phosphorus } \\ \mathrm{S} & \text { Sulfur } \\ \mathrm{Sr} & \text { Strontium } \\ \mathrm{Ti} & \text { Titanium } \\ \mathrm{V} & \text { Vanadium } \\ \mathrm{Y} & \text { Yttrium } \\ \mathrm{Zr} & \text { Zirconium }\end{array}$




\section{PEEK AS A BIOMATERIAL}

\section{Definitions in biomaterial science}

The meaning of a 'biomaterial' has been debated and changed over the years but the European Society for Biomaterials derived the today applied definition in medicine ${ }^{1-3}$. The latest definition is 'a material intended to interface with biological systems to evaluate, treat, augment or replace any tissue, organ or function of the body'4.

In biomaterial science a material is 'a substance of which things are made' and man-made materials are historically divided into metallic, ceramic and polymeric. All these materials have many subdivisions with plentiful applications in medicine ${ }^{2}$. Today biomaterials are used with good success for example in heart valve prostheses, hip replacements, dental implants, intraocular lenses and ventricular assist devices $^{5}$.

Over the past decades remarkable technological improvements in quality, performance and versatility of instrumentation have been achieved using biomaterials when it comes to rehabilitate lost functions and aesthetics. Furthermore with high growth in the geriatric population and rising numbers of hip, knee and teeth replacements, reports have forecasted the biomaterial market to annually grow $13.2 \%$ during $2016-2021^{6,7}$. The global medical device market was in 2016 valued USD 339.5 Billion and projected to reach USD 435.8 Billion by $2020^{8}$ (Table 1 ). 


\section{Basic principles of Polymers}

'Polymer' originates from Greek and means 'many parts' and is a large molecule with many repeated subunits or monomer segments, usually composed of carbon, hydrogen and sometimes oxygen, chlorine and nitrogen ${ }^{9}$. The unique attribute of polymers is determined by the length and composition of the molecular chain ${ }^{10}$.

Table 1: Global medical device technology industry by region (in Billion US\$) 2016-2020. Data obtained from Worldwide Medical Devices Forecast 2020 Report

\begin{tabular}{rrrrrr} 
& \multicolumn{1}{l}{2016} & \multicolumn{1}{l}{2017} & \multicolumn{1}{l}{2018} & \multicolumn{1}{l}{2019} & \multicolumn{1}{l}{2020} \\
\hline North America & 166.6 & 176.5 & 187.3 & 197.9 & 208.6 \\
\hline Asia-Pacific & 68.7 & 72.6 & 77.6 & 82.9 & 88.6 \\
\hline Central/Eastern Europe & 14.6 & 15.7 & 17 & 18.1 & 19.1 \\
Middle East/Africa & 10 & 10.8 & 11.5 & 12.5 & 13.2 \\
Western Europe & 79.5 & 85.1 & 92.6 & 101.4 & 106.2 \\
Total & 339.5 & 360.8 & 386.1 & 412.8 & 435.8 \\
\hline
\end{tabular}

The classification system of polymers can be made based on the molecular forces or the source of origin (Figure 1). Based on the source, polymers can be sub-divided in two groups; natural polymers and synthetic polymers. Natural polymers are abundant in nature and the ultimate natural polymers are the deoxyribonucleic acid (DNA) and ribonucleic acid (RNA) which are the origin of all life. Silk, wool and rubber are other natural polymers. The evolution of synthetic polymers started by the mid-1800s when rubber was accidentally mixed with sulfur which created a more stable rubber that did not deform or melt below 100 degrees Celsius $\left({ }^{\circ} \mathrm{C}\right)$. A few decades later Goodyear was given the patent for vulcanized rubber, the origin of today's car tires. Over the years countless variations of polymers 
have been developed and grown to usage in larger numbers than aluminum, copper and steel industries combined ${ }^{11,12}$.

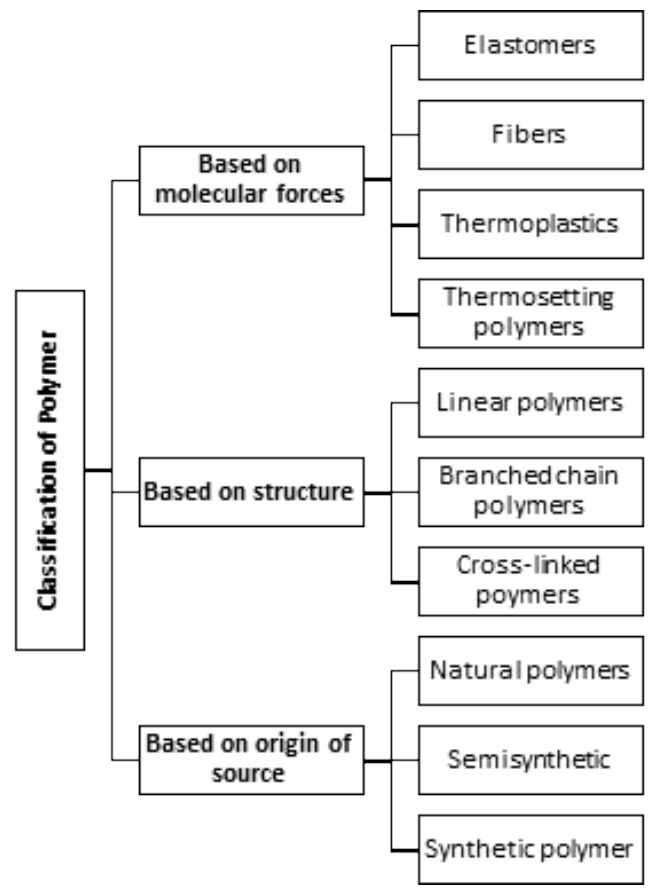

Figure 1. Schematic classification of polymers based on the molecular forces, structure and origin of source.

The inter-molecular forces is another way to differentiate polymers. They can be divided into four types; Elastomers, fibers, thermosetting and thermoplastics (Figure 2). Elastomers have no or very few inter-molecule cross linkages giving an ability to stretch and regain original shape after the force is removed. One example of elastomer is natural rubber. Fibers hold the opposite feature of elastomers with strong hydrogen bonds or dipole-dipole interactions between the chains. In that way the molecules are closely arranged and the polymer possesses high tensile strength and minor elasticity. Nylon and silk are two common examples. A thermoplastic polymer have monomer chains that are networked with weak van der Waals forces and the material soften on heating and set on cooling. Polythene (as 
'plastic') is a common thermoplastic polymer and can be manufactured into any shape and size. In contrast, when heat is applied to a thermosetting polymer the molecules cross-link and permanently set to become a solid component without the ability to re-modulate. Acrylic resin and epoxy are two common thermosetting polymers $^{13,14}$.

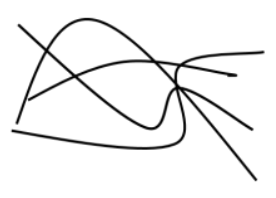

Thermoplastic

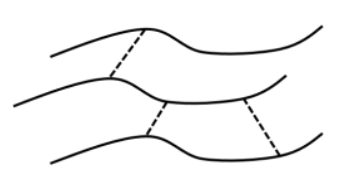

Elastomer

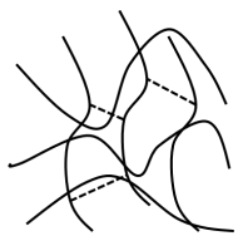

Thermoset

Figure 2. Molecular structure of polymers. The arrangement of the chains determines the properties of the polymer. Branched chains as elastomers and networked thermosets once set have increased strength but less flexibility compared to thermoplastics.

\section{Polymers in the Medical Field}

Rapidly developing science of polymers in medicine was seen during the Second World War where e.g. polyesters and polyamides were introduced for sutures. Since that time considerable research with numerous newly introduced polymers has been conducted and turned the biomedical polymer field into a billion euro industry ${ }^{15}$. The pursuit of constantly improving polymers is motivated by the need for biomaterials in modern therapies and the scientific curiosity to recognize and mimic biological functions with biomaterials ${ }^{16}$.

Polymers for medical devices are most commonly made of polypropylene, polyethylene or poly-vinyl chloride (Table 2). Polymers have a long history from traditional applications such as catheters and syringes to tissue engineering and drug delivery systems. In general, medical polymers can be classified as non-degradable or degradable depending on the reactivity of the backbone molecule upon water exposure ${ }^{17,18}$. Polypropylene is a family of polymers with high rigidity and tensile strength, and good biostability. In medicine it is commonly used as non-degradable sutures. Polyethylene has the advantage to withstand sterilization temperatures and also to possess 
good toughness and wear resistance. These properties make this polymer suitable for drain and catheter tuber and prosthetic joints. PVC is used in short-term applications as the material may become brittle because of leaching plasticizers. Therefore it is used for example as external tubing and bags for blood storage ${ }^{5}$.

This thesis will focus on a non-degradable thermoplastic polymer, polyether ether ketone (PEEK), useful in a wide variety of applications such as orthopedics, dental and tissue replacements ${ }^{10,19-21}$.

Table 2. Polymers in the medical field with their characteristics and some examples of applications.

\begin{tabular}{|c|c|c|}
\hline Material & Characteristics & Applications \\
\hline Polypropylene (PP) & $\begin{array}{l}\text { Semi-crystalline PP holds high rigidity, tensile strength and } \\
\text { biocompatibility }\end{array}$ & $\begin{array}{l}\text { Non-resorbable } \\
\text { sutures } \\
\text { Hernia repair }\end{array}$ \\
\hline Polyethylene (PE) & $\begin{array}{l}\text { High density PE possess good toughness and wear resistance. } \\
\text { Low density PE does not withstand high temperatures and } \\
\text { cannot be sterilized. }\end{array}$ & $\begin{array}{l}\text { Tubing for drains and } \\
\text { catheters } \\
\text { Prosthetic joints }\end{array}$ \\
\hline $\begin{array}{l}\text { Poly vinyl chloride } \\
\text { (PVC) }\end{array}$ & $\begin{array}{l}\text { PVC is a flexible material that is used for disposable and short- } \\
\text { term application products due to the material embrittlement. }\end{array}$ & $\begin{array}{l}\text { Examination gloves } \\
\text { Tubing and blood } \\
\text { storage bags }\end{array}$ \\
\hline $\begin{array}{l}\text { Polydimethylsiloxane } \\
\text { (PDMS) }\end{array}$ & $\begin{array}{l}\text { Normally referred to as silicone. Low glass transition makes the } \\
\text { material very flexible with excellent fatigue resistance. }\end{array}$ & $\begin{array}{l}\text { Breast implants } \\
\text { Heart valves }\end{array}$ \\
\hline $\begin{array}{l}\text { Poly(methyl } \\
\text { metacrylate) (PMMA) }\end{array}$ & $\begin{array}{l}\text { Transparent thermoplastic with the commercial name, Plexiglas. } \\
\text { PMMA has qualities of biocompatibility, ease of manipulation } \\
\text { and low toxicity }\end{array}$ & $\begin{array}{l}\text { Dentures } \\
\text { Intraocular lenses } \\
\text { Bone cement }\end{array}$ \\
\hline
\end{tabular}

\section{Properties of PEEK Polymers}

Preparation of PEEK was first reported by Attwood et al. ${ }^{22}$ and the crystalline unit cell was presented with a chemical structure of an aromatic backbone molecular chain with ketone and ether as interconnected functional groups (Figure 3). The first successful synthesis of PEEK was through a nucleophilic displacement where a diphenyl sulfone was used as solvent ${ }^{22}$. PEEK becomes solid at room temperature and the glass transition take place at $143^{\circ} \mathrm{C}$. The melt transitions occur at $343^{\circ} \mathrm{C}$. In room temperature and polymerized state the polymer is inert to chemicals and is only soluble in $98 \%$ sulphuric acid $\left(\mathrm{H}_{2} \mathrm{SO}_{4}\right)^{10,23,24}$. The chemical composition provides the polymer with properties such as stability at high temperatures, resistance to chemical and radiation damage (Table 3). As a result of these high performance properties, PEEK has been employed in challenging industrial applications in aerospace and automotive engineering ${ }^{25,26}$. By the late 1980 s the medical field gained interest for 
this material in the field of trauma and orthopedic surgery. Originally used as a spinal implant, PEEK is today commonly used as spinal fusion cages ${ }^{27,28}$.

Table 3. Physical properties of PEEK OPTIMA LT1. Data obtained from Invibio Biomaterial Solutions.

\begin{tabular}{lrr}
\multicolumn{1}{c}{ Property } & Values & Unit \\
Tensile Strength & $90-100$ & $\mathrm{MPa}$ \\
Young's Modulus & 3.6 & $\mathrm{GPa}$ \\
Elongation @ break & 50 & $\%$ \\
Glass Temperatue $\left(\mathrm{T}_{\mathrm{g}}\right)$ & 143 & ${ }^{\circ} \mathrm{C}$ \\
Melting point & 343 & ${ }^{\circ} \mathrm{C}$ \\
\hline
\end{tabular}<smiles>COc1ccc(Oc2ccc(C(=O)c3ccc(C)cc3)cc2)cc1</smiles>

Figure 3. Chemical formula of PEEK with a semi-crystalline aromatic polymer consisting of repeating units. The molecule is relatively stiff but with freedom to rotate around the ether (-O-) bonds and ketone-carbon bonds (-CO-). Figure from Blundell and Osborn ${ }^{\mathrm{I}}$.

\section{Processing and Thermal Transitions}

Crystallization of polymers is a dynamic transition from an amorphous state where the molecule chains are highly disordered, into a folded and ordered molecule state, called lamellae ${ }^{29}$. PEEK is a semicrystalline polymer with relatively strong intermolecular forces that makes the material firm even at the glass transition temperature (143 $\left.{ }^{\circ} \mathrm{C}\right)$. Producing a $100 \%$ crystalline polymer is in practice impossible since the molecules have to fold into arrangement from the highly 
disordered state. The molecular chain is composed of crystalline lamellas surrounded by amorphous regions and in straightened state the molecule can be several hundreds of meter long ${ }^{30}$ (Figure 4).

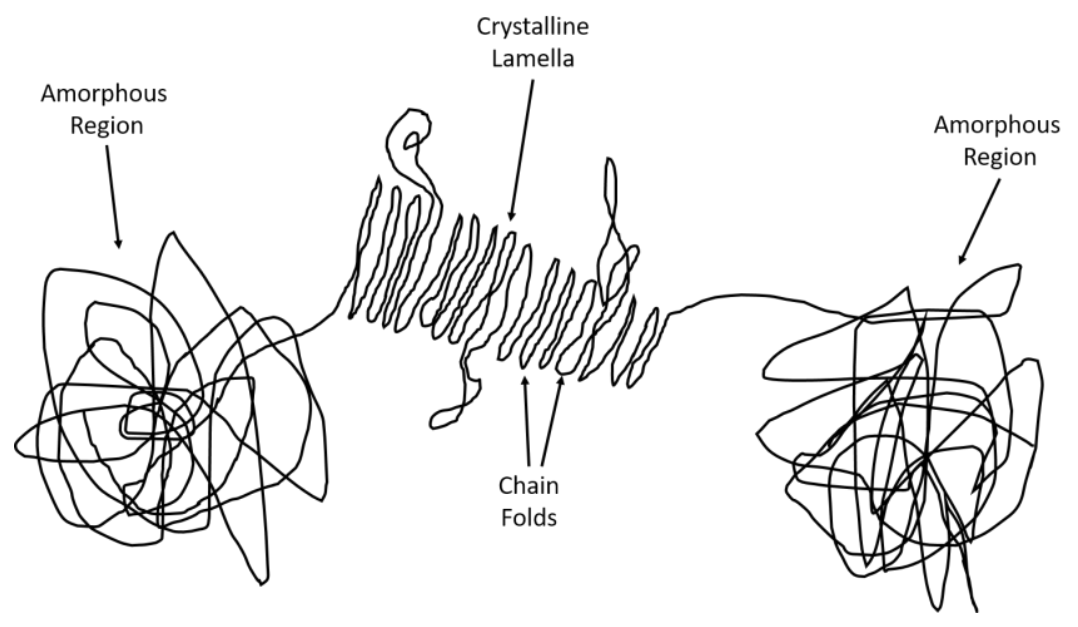

Figure 4. Schematic representation of polymer chains with repeating units of amorphous and crystalline regions.

PEEK has an advantage over other polymers due to its high degree of crystallinity which increases its mechanical strength and decreases the risk of stress cracking ${ }^{22}$. The crystalline structure of PEEK is built up by many spherulites. Dimensions and arrangement of these spherulites depends on the processing conditions and forgoing thermal history which affects the crystallinity of the polymer ${ }^{31-33}$. In general, the thickness of the lamellae is in the angstrom range (50-60 $\AA$ ) and the spherulites are $25-40 \mu \mathrm{m}$ in diameter ${ }^{30}$. PEEK as a semicrystalline polymer can be processed with different level of crystallinity depending on the processing conditions after the melt stage. If the melt is cooled rapidly, a glassy or amorphous state is achieved whereas a slow cooling develops a higher level of crystallinity ${ }^{30}$. When the melt is cooled the arrangement of the spherulites transforms from an amorphous state into a more ordered state. When reheated, the polymer will return to an amorphous state ${ }^{33}$. This occurrences is illustrated in figure 5 where the rate of crystallization is dependent on the temperature. 


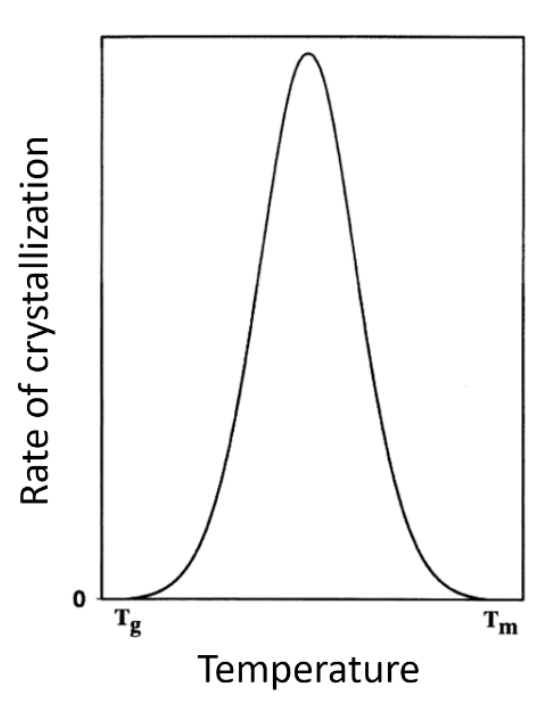

Figure 5. Typical form of crystallization rate-temperature curve for a crystalline polymer.

\section{Mechanical properties}

The mechanical properties and behavior of a polymeric material are complex and can be divided into many subgroups. In this chapter the focus will be on the properties that are favorable or harmful for the use in medicine.

The following material properties are commonly evaluated in biomaterials science:

1. Stiffness - Resistance to deformation like elongation, twisting, bending etc.

2. Strength - The ability to resist force or pressure before rupturing

3. Toughness - The absorbed strain energy under static, impact and fatigue loading until material fracture

4. Wear resistance - The ability to withstand loss of material upon contact friction 
One of the major reasons for the medical field to gain interest into PEEK was its closely matched stiffness to bone. Orthopedic appliances as spinal cages, compression bone plates and hip prostheses were usually made from metallic alloys where the stiffness was 1020 times that of cortical bone ${ }^{34}$. This mismatch in stiffness may cause a stress shielding and may lead to bone resorption ${ }^{35-37}$. The bone resorption around the inserted device may cause loosening of the implant or weakening of the bone and eventually fracture. The use of polymer devices with a similar stiffness as cortical bone has, in comparison to metallic alloys, demonstrated a more favorable stress distribution to the surrounding bone ${ }^{38,39}$. According to Wolff's law, the bone will adapt to the exposed load. Therefore, the density of the bone will be dependent of the stress distribution ${ }^{40}$. PEEK can be tailored to the stiffness required for the application with addition of carbon reinforced fibers (CFR, Figure 6). CFR-PEEK can with continuous fibers achieve excellent stiffness, strength and durability in order to match the implant bed ${ }^{41,42}$. In addition, CFR-PEEK in a total hip model with saline, have demonstrated excellent compression strength durability ${ }^{43}$.

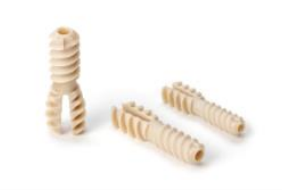

Hammertoe implant

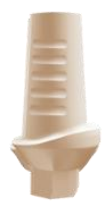

Implant abutment

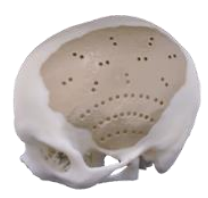

Skull reconstruction

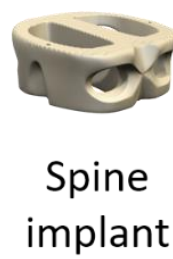

Figure 6. Different clinical applications of PEEK that are currently used in dental, orthopedic and reconstructive applications. 
To understand the failure mechanisms of PEEK implants in spinal and dental applications, the strength of the material in static and cyclic loading conditions has to be assessed ${ }^{10}$. The strain rate and temperature in general decide the mechanical behavior of neat PEEK but it can also be changed by the molecule weight and size and orientation of the crystallized lamellae $e^{44-48}$. In addition, an increase in crystallinity has been found to decrease the toughness ${ }^{49}$. In room temperature, PEEK shows a linear elastic deformation at small strains and compressions (Figure 7). After the yield transition caused by strain or compression, the behavior varies depending on the temperature and rate of strain. Until material failure after large deformation, the behavior is difficult to characterize due to a change in molecular alignment. However, PEEK as a biomaterial is expected to operate in body temperatures $\left(35-44^{\circ} \mathrm{C}\right)$ where the material is present no structural deformation ${ }^{46,47}$.

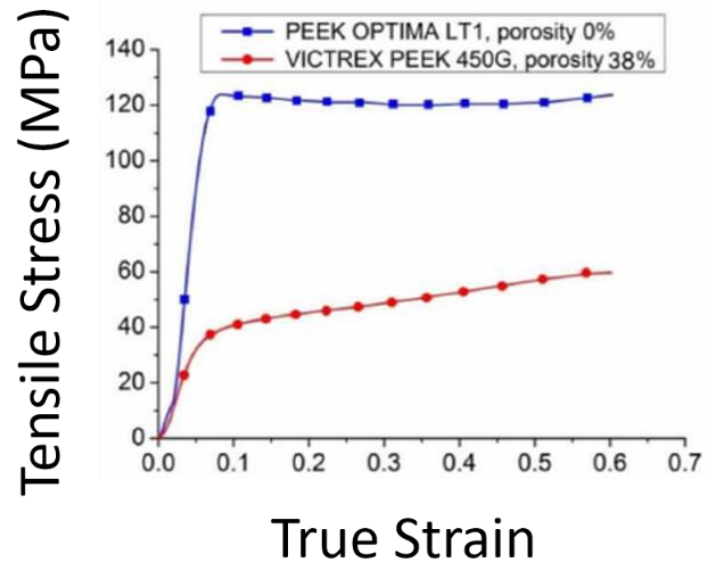

Figure 7. Strain-stress plots for PEEK-OPTIMA and Victrex 450G with $0 \%$ and $38 \%$ porosity, respectively. Figure obtained from Vaezi and Yang.

Neat PEEK has a high wear resistance and friction coefficient $(\mu \geq$ 0.4 in dry sliding) in addition to a low thermal conductivity. These features has gain interest for PEEK arthroplasty bearing surfaces. In 
total hip replacement a common reason to failure is a result of aseptic loosening ${ }^{50}$. Wear debris and stress shielding are believed to cause the failure. PEEK has been evaluated in several studies with respect to contact pressure and cross-shear ratio in relation to the wear of orthopedic materials ${ }^{51,52}$. Ultra-high-molecule-weight polyethylene (UHMWPE) and cobalt-chromium-molybdenum (CoCrMo) provided more wear particles in a wear-test model compared to CFRPEEK. A study by Howling et al..$^{53}$ evaluated wear debris from CFRPEEK against alumina. The wear particles from PEEK in an in vitro culture model showed no cytotoxic effects on fibroblast cells (L-929) or a human hematopoietic cell line (U-937). Other authors suggested that PEEK wear particles have no adverse tissue reaction since it has no cytotoxic effect on cells in culture ${ }^{10,53}$. An in vivo study of PEEK applied in a total hip system (Stryker SA, Montreux, Switzerland) was evaluated in 30 patients after 3 years in function no implant was replaced due to aseptic loosening ${ }^{54}$. However, this is not a strikingly different outcome in comparison to the norm when placing ordinary types of hip arthroplasties.

\section{Chemical and radiation stability}

The chemical structure of PEEK is composed of aryl rings interconnected via ketone and ether groups opposite each other. This structure creates an exceptional resistance and un-reactivity to chemicals but also against thermal and irradiation degradation. In addition, the PEEK structure can only be dissolved by sulfuric acid. Altogether, this may explain the excellent biocompatibility of PEEK.

PEEK can endure extreme conditions with different liquids but as an implantable biomaterial the resistance in water is of particular interest. PEEK has a maximum solubility in water and brine of $0.5 \% \mathrm{w} / \mathrm{w}$ at $75^{\circ} \mathrm{C}$ and is independent of the salinity of the exposed liquid ${ }^{45,55}$. The results by Boinard and colleagues ${ }^{45}$ showed that the small water uptake altered the degree of crystallinity at the surface which did not re-form after the samples were dried. A study by Liebermann et al. ${ }^{56}$ evaluated the physicomechanical properties of PEEK among other polymers after aging in different liquid substances. The aim of the study was to elucidate the physicomechanical knowledge of PEEK 
as a material to support fixed dental prostheses. The authors recommend the material for long-term oral restorations since it shows as low solubility and absorption rates as do other traditional PMMAbased materials.

The thermal stability of PEEK in a clinical application is of no concerns since the temperature never exceeds the body temperature ${ }^{57,58}$. However, when the material is sterilized and exposed to pressurized steam (autoclave) the temperatures exceeds the body temperature. However, no significant reduction in flexural strength was revealed on CFR-PEEK after 100 autoclave cycles ${ }^{59}$. Minor changes in the microstructure have been demonstrated but the bulk material remains unaffected following steam sterilization ${ }^{60}$. If steam sterilization is to be avoided another sterilization process is the gamma radiation. Some polymers (UHMWPE) are sensitive to gamma radiation where breaking of chemical bonds may cause a formation of highly reactive free radicals ${ }^{61}$. Even after radiation, these free radicals may react and influence the surrounding material and tissue. However, several aromatic polymers (such as PEEK) were exposed to gamma radiation under oxygen pressure to evaluate the changes in tensile properties ${ }^{62}$. The effect of the radiation dose was estimated by calculating the elongation at breakage which was found to be as low as $10-20 \%$ of the dose from electron-beam radiation. The authors concluded that the altered mechanical properties of the deterioration was caused by degradation of the polymer main chain (chain scission).

To summarize, PEEK has been found to perform extremely well in many environments thanks to its stable chemical structure. Radiation and aqueous exposure are of no concern and in applications as a biomaterial where the temperature is around $37^{\circ} \mathrm{C}$ PEEK performs impeccably.

\section{Clinical applications}

The mechanical drawbacks of metal alloys inspired researchers in the late 1980s to conduct studies on PEEK polymers ${ }^{27,28,34,63}$. PEEK was by that time used in the automotive- and aerospace industry due to its chemical resistance, light weight and mechanical advantages 
compared to metals. In 1998, Victrex® launched its PEEK-OPTI$\mathrm{MA}^{\mathrm{TM}}$ for medical implant use and since then research and developments have been in progress in almost every area of application where metal alloys are presently being used. Interest was from the beginning specially gained in orthopedics, spine and trauma surgery but are currently under evaluation in new fields such as dentistry and cardiac surgery ${ }^{42,64-66}$.

\section{Spinal implants}

Patients diagnosed with a degenerative disc in the lumbar spine can be treated by a spinal fusion, a surgery designed to end the low back pain caused by motions of the vertebra. There are two main approaches to spinal fusion; posterolateral interbody fusion (PLIF) and anterior lumbar interbody fusion (ALIF) ${ }^{67-69}$. The main differences between the two approaches are the access point and the site where the bone graft is inserted in the spine.

The PLIF technique used a pedicle screw fixation (Figure 8). Two screws are placed within the pedicle of each vertebral segment, one of each side of the spine. Each vertebra is connected with a metal rod. The bone graft is inserted between the transverse process in posterior side of the spine which allows bone fusion of the processes from one vertebra to another ${ }^{67}$. 


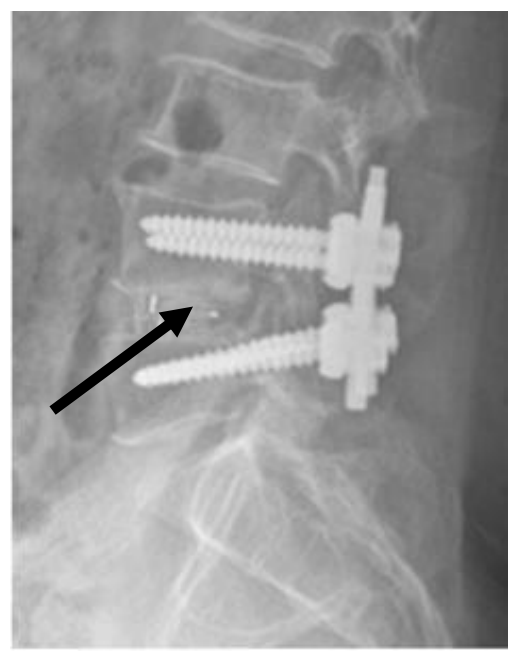

Figure 8. Lateral radiograph at 12 months post-operative showing a PEEK cage implanted between the vertebras to enable a bone fusion. Figure adapted from Lin et al ${ }^{\mathrm{III}}$.

The ALIF procedure is similar to the PLIF except that the spine is approached through the abdomen. This technique provides access to the vertebra body and the disc. After the disc is removed, the intervertebral space is filled with a spinal implant and bone graft (Figure 9). Compared to the PLIF, this technique provides an increased surface area with a more favorable bone fusion but the morbidity of the patient is higher due to the risk of injuries of the nearby large blood vessels (Vena Cava and Aorta).
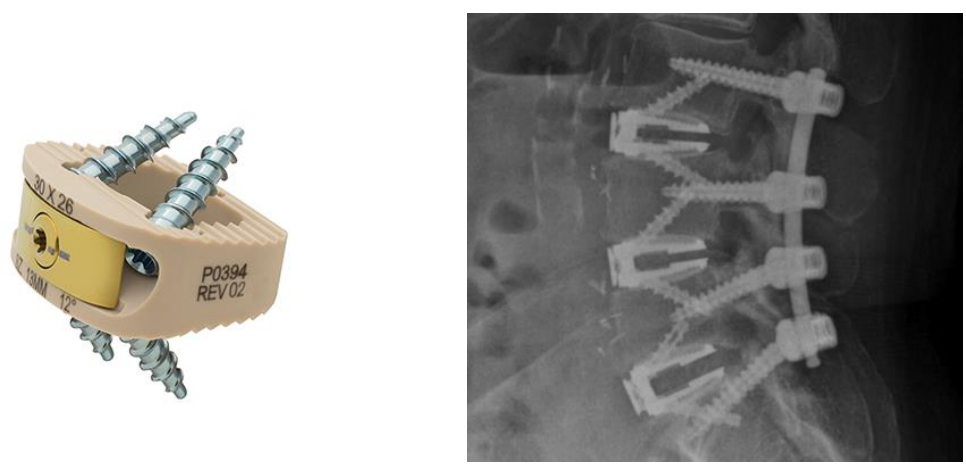

Figure 9. Anterior PEEK cage in combination with posterior fixation. Image obtained from Mobbs et al. ${ }^{\text {IV }}$ 
Titanium and stainless steel have provided satisfying long-term results due to their stiffness and rate of osseointegration. However, the stiffness also becomes a disadvantage because the mismatch to bone which may lead to adjacent segment degeneration (ASD). Post-operative examination by conventional X-ray is difficult to interpret because the metal streak artifact caused by the implants.

In 2001 the Food and Drug Administration (FDA) approved the CFR-PEEK for lumbar fusion. At this time, from surgical experience, the spinal fusion was performed with stand-alone threaded cages and the protocol of CFR-PEEK was described in a monograph by a surgeon inventor, John Brantigan ${ }^{70}$. CFR-PEEK provided similar elastic modulus as the human bone and the in the post-operative evaluation the transparency to X-ray enhanced the interpretation ${ }^{71-}$ 73. The Brantigan cage was intended to be inserted with an ALIF approach together with posterior instrumentation in order to enhance the stability, a so-called $360^{\circ}$ fusion (Figure 10). Studies have confirmed the superiority of the Brantigan cage approach compared to the posterolateral lumbar fusion ${ }^{74,75}$. According to several studies, the radiolucency of PEEK has facilitated the radiographic assessment of the fusion ${ }^{76-78}$. However, there are potential complications for PEEK cages, as with any implant in load-bearing situations ${ }^{79,80}$. Subsidence caused by wear debris and fracture of the implant cage has been documented by some authors ${ }^{81,82}$.

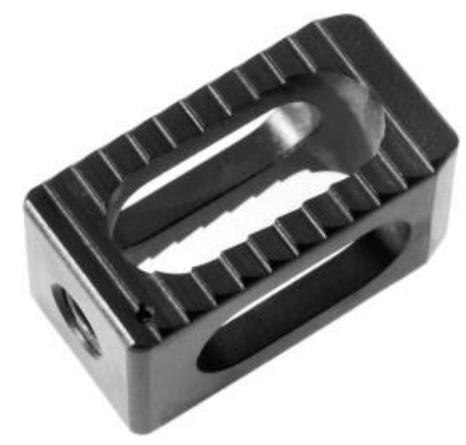

Figure 10. The original design of an interbody spine implant made of carbon-fiber-reinforced polymer. Image from Brantigan et al. ${ }^{\mathrm{V}}$ 


\section{Total joint arthroplasties}

As mentioned before, PEEK is resistant to fatigue strain, retains mechanical properties in the range of human bone while it is radiologically transparent which makes it suitable for a range of orthopedic applications. In the 1990s, PEEK and CFR-PEEK were introduced as load bearing implants for total joint arthroplasties (TJA ${ }^{83}$. In a TJA the load is applied to a femoral stem and the forces are spread to the surrounding bone and tissue (Figure 11). If the femoral stems are made of metal, the load to the surrounding tissue may be decreased. This is called stress shielding and the biological response is a slow resorption and re-distribution of the adjacent bone. This may potentially lead to implant loosening ${ }^{84}$. However, an implant failure usually occurs by a combination of several mechanisms ${ }^{85}$. Wear particles from the implant surface may induce an inflammation which may cause an aseptic loosening of the implant ${ }^{86,87}$. The biological response to wear particles from PEEK in different joint replacements have been studied in several publications and it is generally accepted that phagocytozable particles $(0.1-10 \mu \mathrm{m})$ are the ones with potential cytotoxic effect ${ }^{88,89}$. The particles can stimulate macrophages to produce pro-inflammatory and osteolysis mediators that may cause aseptic implant loosening. Not until the characteristics of the PEEK particles are identified and repeatable analyses would it prove possible to analyze the biological response ${ }^{90}$. 


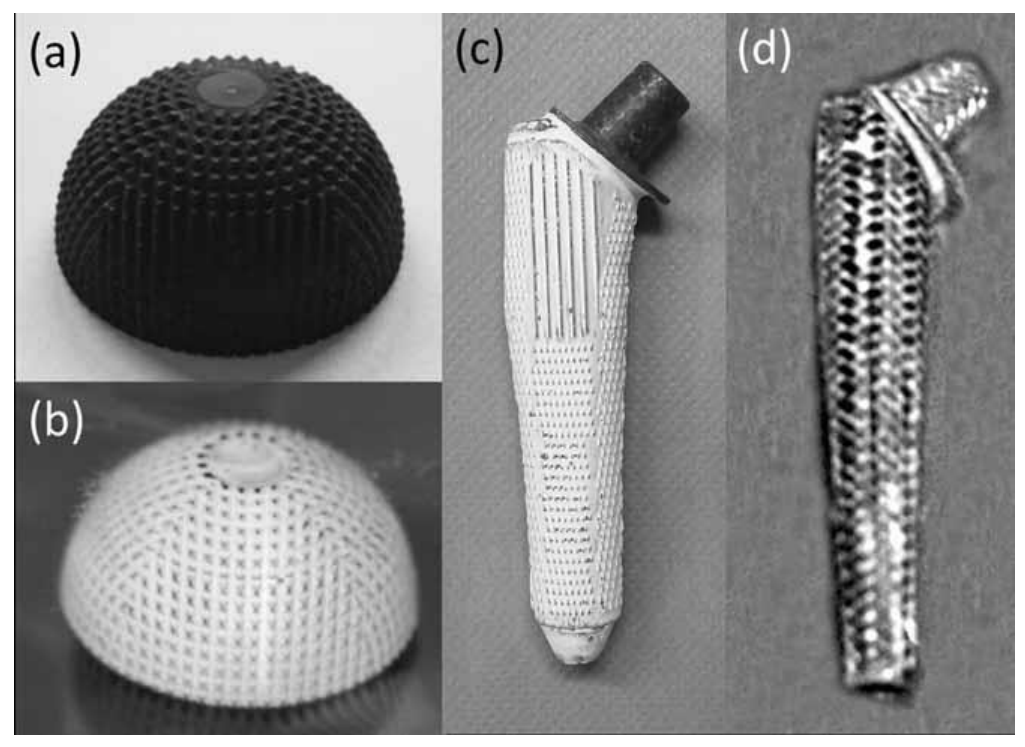

Figure 11. CFR-PEEK has gained interest in the use for orthopedic implants stems due to its tailored mechanical properties, chemical stability and less tendency of wear debris. A: Cemented CFR-PEEK cup. B: Cementless CFR-PEEK cup. C: Cementless CFR-PEEK stem. D: Cemented CFR-PEEK stem. Image obtained from Nakahara et al. ${ }^{\mathrm{VI}}$

\section{Trauma implants}

The clinical outcomes over the last decades of trauma implants and fracture fixation has motivated a paradigm shift ${ }^{91-93}$. From a total fixation with no mobilization to a more flexible fixation. A too rigid fixation may cause an incomplete bone healing with prolonged rehabilitation time. Consequently, a more flexible fixation has been introduced where the stress from the loading is distributed more evenly to the surrounding structures. PEEK holds all the qualification that makes it suitable for fracture fixation. It is biocompatible, resistant to solvents and the stiffness is close to that of human bone. The stiffness can be further adjusted by adding carbon fibers to the compound (CFR-PEEK). Since PEEK is a thermoplastic polymer, the shape can be designed and adjusted at the surgical procedure ${ }^{10}$. PEEK also provides radiographic evaluation without artifacts which enables a good visualization of the newly formed bone ${ }^{94}$. Despite all 
these advantages, only a few clinical reports of the performance of PEEK have been published. A biomechanical study by Schliemann et al. ${ }^{95}$ found that PEEK did not provide satisfactory fixation strength when compared to titanium. The same authors published a 2-year prospective clinical study, comparing PEEK and conventional locking plates for humeral fractures ${ }^{94}$. The fixation with PEEK plates was found comparable to the conventional locking plates. Micromotion is nowadays accepted, but only in the axial direction ${ }^{96}$. Due to the flexibility of PEEK, the bending, torsional and shear forces are not sufficiently stabilized in fracture fixation. Furthermore, PEEK is under consideration in cranioplasty where CT-guidance and $\mathrm{CAD} / \mathrm{CAM}$ (computer-aided design and computer-aided manufacturing) techniques provide a precise and time-saving reconstruction $^{97}$. However, metal locking plates are still dominant in the trauma field, therefore PEEK needs further studies and clinical reports to increase its practice.

\section{Dental applications}

Titanium has since the 1960s been the most common choice of material in oral endosseous implants due to its superior mechanical properties and biocompatibility and titanium constructions have resulted in increased quality of life for patients ${ }^{98-101}$. However, titanium and titanium alloys (such as $\mathrm{Ti}_{-6} \mathrm{Al}_{-7} \mathrm{Nb}$ and $\mathrm{Ti}_{6}{ }_{6} \mathrm{Al}-4 \mathrm{~V}$ ) have over the years demonstrated several clinical complications. One problem is the potential hypersensitivity or allergy to titanium from degradation products ${ }^{102-105}$. Another problem is the divergent elastic modulus to the surrounding bone which may cause adverse stress at the bone interface ${ }^{106}$. In esthetic anterior cases where the patient have a high smile-line, the dark color of the titanium implant may shine through the tissue ${ }^{107,108}$. All in all, there may be a need for an introduction of an alternative to metal implants. Ceramic implants were introduced as an alternative to titanium with tooth-like color and good biocompatibility. However, zirconia suffers with similar deviation of elastic modulus compared to bone as does titanium ${ }^{109}$. 
PEEK may be another alternative with superior elastic modulus for bone applications, excellent biocompatibility and tooth-like appearance $^{27,110}$. Suggested dental applications for PEEK includes; dental implants, implant abutments, fixed bridges and crowns and removable dentures and components (Figure 12).

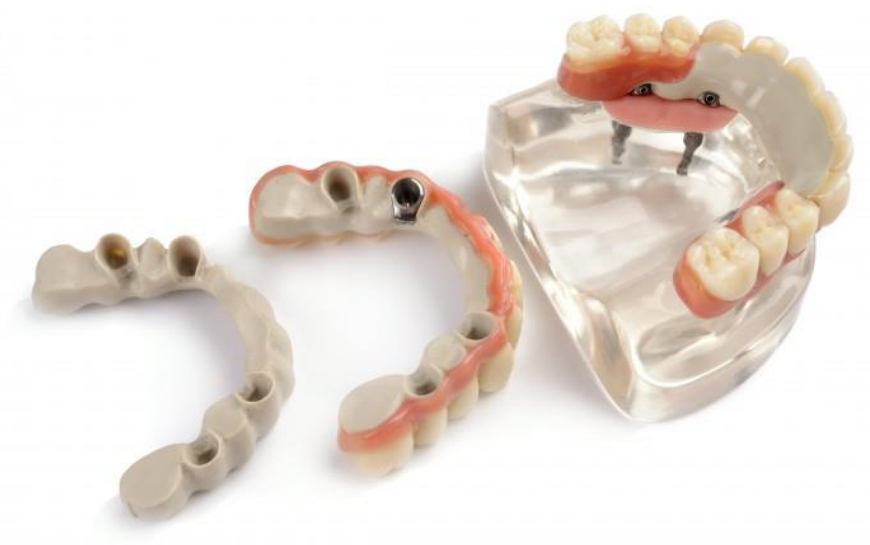

Figure 12. Using subtractive process, full and partial dentures and overdentures, crowns and bridges can be manufactured. Image from Juvora Dental Innovations.

As for dental implants, studies have presented different conclusions regarding stress shielding and biocompatibility. Lee et al. ${ }^{64}$ concluded that PEEK implants compared to titanium may reduce stress shielding while Sarot et al. ${ }^{42}$ suggested that there is no difference between the stress distributions. Therefore, no general conclusions on stress shielding can be made on the present knowledge. Neat PEEK is hydrophobic and bioinert ${ }^{111,112}$, even though some studies have found no differences between the osseointegration between PEEK and titanium or zirconia ${ }^{113,114}$. Numerous modifications have been applied to improve the biological properties of PEEK. Further readings on this topic will be described later in this introduction. However, as far as the author knows, there are no PEEK dental implant systems marketed. 
Implant abutments made of PEEK have been evaluated in a controlled clinical trial (RCT) by Koutouzis et al. ${ }^{115}$ and revealed no significant difference on inflammation and bone resorption compared to titanium abutments. In terms of bacterial adhesion, PEEK abutments has demonstrated comparable results as those made of titanium and zirconia ${ }^{116}$. Santing and co-workers ${ }^{117}$ compared temporary PEEK abutments with 2 commercial systems on fracture strength and failure mode. No differences between the systems were found except for anterior maxilla where PEEK showed significantly lower fracture strength. Similar results were discovered by Rubén et al. ${ }^{118}$ with less fracture resistance compared to titanium but the elastic deformation was significantly less than titanium.

The properties of PEEK has inspired dental innovators to improve the components in removable and fixed dental prostheses and crowns. Since PEEK can be manufactured by using CAD/CAM there are no design limitations. A study comparing retention clasps of PEEK and Cobalt Chromium (CoCr) demonstrated less retention for PEEK cf. the metal ${ }^{119}$. However, the authors believes the retention of PEEK clasps to be sufficient for clinical use. A clinical report on a maxillary obturator prostheses made of PEEK for a patient with maxillary defect provided the patient with a more functional and lighter prostheses compared to the conventional alternatives ${ }^{120}$.

Using PEEK as cemented crowns requires a surface treatment. A study evaluating shear bond strength after sulfuric acid etching found more differences due to cement type than the etching time. However, the results seems to be promising but the authors suggested further studies on other cement systems ${ }^{121}$. A fixed dental bridge (FDP) comparison of CAD/CAM manufactured and conventional pressed from granulae was evaluated based on the load-bearing capacity ${ }^{122}$. The study summarize that the CAD/CAM manufactured FDPs showed advantages over the pressed granular PEEK FDPs.

In conclusion, PEEK is a newcomer in the dental field and the published data is limited. However, the results show promising abilities 
and possible future applications. Therefore, more research and clinical trials are needed to discover indications for the material in future dental applications.

\section{Biocompatibility and bioactive modifications of PEEK}

\section{Definitions}

Immediately when a biomaterial is inserted into a living host, interactions take place between the host and the biomaterial. The word "biocompatibility" refers to the ability of a material to perform with an appropriate host response in a specific situation" ${ }^{123}$. Biocompatibility is not a property of a material itself. It should be seen more like an interaction between the host and the biomaterial. Within the same host, a biomaterial could be biocompatible in one application but cytotoxic in another. Minimum requirements for implanted biomaterials is that they should be non-toxic, non-mutagenic and noncarcinogenic.

The biological performance of materials and living systems may be viewed in two ways ${ }^{124}$ :

1. The host response - the local or systemic response to the implanted material such as macrophage proliferation or fibrous encapsulation

2. The material response - the material reaction to the living system such as corrosion or water absorption

\section{Cytotoxicity and genotoxicity of bulk PEEK}

There has been a considerable amount of research on bulk PEEKOPTIMA and CFR-PEEK-OPTIMA to meet the FDA requirements for biomaterials ${ }^{34,63,125-127}$.

Wenz et al. ${ }^{63}$ were the first to recommend PEEK for further in vivo studies after showing the in vitro cellular response to PEEK to be insignificant in terms of cytotoxicity. Using a MMT assay and an ALP kit the metabolic activity of cells can be measured and related to the level of cytotoxicity of a given material. In a study by Macnair and collegues ${ }^{128}$, PEEK showed insignificant toxicity on preserved rat osteoblasts. More similar studies demonstrated similar results 
and all concluded PEEK to be a suitable material for bone applications ${ }^{126}$. The attachment and proliferation of osteoblasts and fibroblasts were assessed by Hunter et al. ${ }^{129}$. Present and prospective orthodontic biomaterials as PEEK, titanium, cobalt chrome molybdenum alloy and UHMWPE were evaluated and compared in a series of culture experiments. The results demonstrates that PEEK had no deleterious effect on the exposed cells and the attachment as well as proliferation was significantly enhanced compared to UHMWPE. Katzer et al. ${ }^{127}$ evaluated the cytotoxicity and mutagenicity of PEEK using Ames test on different bacterial Salmonella typhimurium strains. The bacteria produced no mutagenic or cytotoxic activity on PEEK. Therefore the authors suggested further experimental testing on animals.

\section{Animal studies of bulk PEEK}

Orthopedic implant system may generate wear debris after years in function which is believed to be one potential reason for aseptic implant loosening ${ }^{130}$. To evaluate a new spinal system, made of metal and PEEK, a worst case scenario was created where 50 million particles per site was injected into the spinal canal of a rabbit spine ${ }^{131}$. This experimental model allows evaluation of the inflammatory response through histology of the neural tissues of the rabbit at 1,4 and 12 weeks post-surgery. The results showed normal vascularization in the spine and the initial inflammations was decreased over a 12 -week period. At the late time-point an increase in connective thickness was discovered as a particle encapsulation. In conclusion by the authors, the PEEK particles was well tolerated by the neural tissues.

PEEK interaction with soft tissue was evaluated by Jockisch et al. ${ }^{132}$ where PEEK and CFR-PEEK cylinders were inserted into the vertebral muscles of a rabbit. Histopathological evaluation was carried out after 8 and 12 weeks of healing. In proximity of the cylinders no visible infection or adverse tissue responses were observed. The histological sections revealed a mild chronic inflammation and few macrophages and giant cells.

In orthopedic applications, implant debris is generally released after years in function and the particles are believed to be one reason for aseptic loosening. A comparative study on PEEK-OPTIMA and 
UHMWPE with respect to macrophage response to three different particle sizes was performed by Hallab and co-workers ${ }^{133}$. No significant difference was found on macrophage viability or proliferation but a greater cytotoxicity responses to UHMWPE than PEEKOPTIMA was observed. Conclusion was made that PEEK-OPTIMA particles were more biocompatible than UHMWPE for this application.

The biocompatibility of a biomaterial in bone, osteocompatibility, is currently the most common situation for PEEK evaluations. In a sheep model, PEEK interbody fusion devices were inserted in the spine and subjected to radiographic and histologic evaluations at 5 months post-surgery ${ }^{134}$. There were no signs of degradation or wear debris. The degree of bone mineralization inside the fusion chamber was found to be normal and comparable with the conventional metal cages. The authors suggested PEEK to be a useful material for interbody fusion cages due to the radiolucency, excellent biocompatibility and decreased stiffness ${ }^{134}$.

\section{Bioactive modifications of PEEK}

PEEK is considered to be a relatively bioinert material but cannot achieve adequate osseointegration after implantation for load bearing applications ${ }^{135}$. As mentioned earlier, PEEK holds excellent mechanical properties for orthopedic and dental applications but it lacks sufficient bioactive properties. Therefore, during in the last decades there have been large number of studies to evaluate diverse methods to improve PEEK with respect to its ability to osseointegrate. This chapter is divided into the 2 methods of improving the bioactivity, surface modification and altering the composition of the bulk material.

\section{Bioactive PEEK composites}

A composite material is made from two or more constituent materials where the properties combined provide a new material characteristics, different from the original materials. In polymer science with the intention to improve the bioactivity of a material, calcium phosphates such as HA, $\beta$-tricalcium phosphate ( $\beta$-TCP), calcium silicate (CS) and bioglass are common additives ${ }^{136-138}$. Strontium- 
substituted HA has also been investigated ${ }^{139}$. These additives are selected due to their ability to bond to living bone and are commonly used in bone augmentation with successful clinical outcomes ${ }^{140-143}$. Alone, these additives possess insufficient mechanical properties as an implant but incorporated with PEEK new favorable mechanical properties can be created ${ }^{136,140}$. The past and current research on PEEK composites have utilized beads and powder from the FDA approved polymers by Victrex (150XF, 150PF and 450G) and PEEKOPTIMA® (LT1PF and LT3UF) $)^{138,139,144-158}$.

Bioactive PEEK composites with an impregnating particle size $>100 \mathrm{~nm}$ is not on the nano scale and, therefore classified as "conventionally sized particles". Since PEEK possesses excellent mechanical properties, several studies have evaluated the mechanical effects of HA incorporated into PEEK composite ${ }^{10,146,159}$. The mechanical properties were evaluated after varying the volume percentage of HA. By progressively adding more HA, the tensile modulus and microhardness increased while strength and fracture resistance decreased. Findings indicated that $30 \mathrm{vol} . \%$ is the optimal ratio where the mechanical properties are similar to human bone ${ }^{159}$ (Figure 13). Another study from the same research group found evidence of a microscopic interfacial failure between the HA filler and PEEK matrix which may initiate and cause fractures ${ }^{146}$. Similar findings of poor interfacial adhesion have been published by several authors $^{138,145,155}$. 


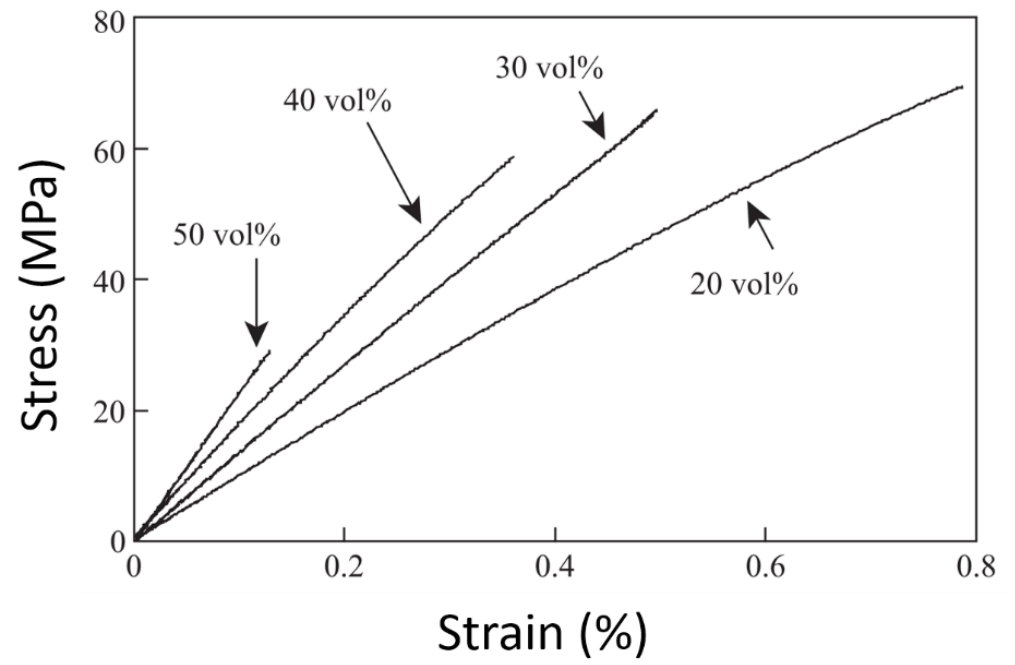

Figure 13. Typical strain-stress curve of PEEK reinforced with 20, 30,40 and $50 \%$ vol. \% HA whiskers. Figure from Converse et al. ${ }^{\mathrm{VII}}$

An in vitro study, Zhang and co-workers ${ }^{160}$ evaluated the biocompatibility of HA reinforced polymers by selective laser sintering. The osteoblast cell attachment, morphology, proliferation and differentiation was monitored on primary human osteoblast cells. The amount of HA was found to positively affect the osteogenic response to the composite. The same technique evaluating different vol. \% of HA was applied by Tan et al. ${ }^{147,148}$ The microstructure and the bioactivity of the formed composite was evaluated and the technique showed promising results. Several studies have confirmed cell attachment (fibroblasts, human osteoblasts and human fetal osteoblasts) on bioactive PEEK composite ${ }^{151-153}$. However, Wong et al. ${ }^{139}$ found no differences in the proliferation or differentiation of osteoblasts for PEEK, HA-PEEK and Sr-PEEK. In contrast, another in vitro study demonstrated that an HA-coated surface compared with a plastic surface, increased osteoblast differentiation but suppressed their growth ${ }^{161}$.

In simulated body fluid (SBF), the ability to form apatite can be examined using ion concentrations close to human blood plasma. A study by Kokubo et al. ${ }^{162}$ demonstrated a material that formed apatite on its surface in SBF also produced it on a surface in the living 
body ${ }^{162}$. However, this relationship can only be applied when there is no cytotoxic or allergic reaction to the material. An in vitro study by $\mathrm{Yu}$ et al. ${ }^{149}$ evaluated the bone-bonding ability of HA-PEEK with different HA vol. \%. The amount and speed of deposited bone on the surface was found to be in direct correlation with the HA vol. \% in the matrix. Samples without HA incorporated showed no significant change in apatite formation in SBF.

The amount of in vivo studies on osseointegration of bioactive PEEK composites are significantly fewer than those in vitro. Bakar and colleagues ${ }^{138}$ developed a new bioactive PEEK composite with a high amount of HA (up to $40 \mathrm{vol} . \%$ ) and a porosity of $60 \%$ using a series of processes (compounding, granulating and injection molding). To obtain mechanical properties in the regime of cortical bone and ensure a maximum exposure of HA particles, the PEEK composite should contain 20-30 vol. HA \%. Histological findings from this in vivo study revealed in the early stages of implantation an activity of fibroblast promoting vascularization. After longer healing periods (16 weeks) a mature bone was formed due to an increased osteoblastic activity.

Other materials apart from HA have been demonstrated to improve the bioactivity of PEEK. Strontium salts have been found, both in vivo and in vitro, to stimulate formation and inhibit resorption of bone $^{163}$. An in vitro comparison between Sr-HA-PEEK and HAPEEK was performed by Wong and co-workers ${ }^{139}$. With respect to apatite formation, the addition of Sr-HA significantly increased the bioactivity. In terms of cell proliferation and ALP activity, there were no differences between the two compared materials. Calcium silicate reinforced PEEK (0-50 vol. \%) was found to promote apatite formation in an SBF composition, with an exception for pure PEEK ${ }^{156}$. An increased CS content decreased the required time for apatite formation, the optimum content was 20 vol. \% for this actual study. Glass fiber and PEEK were evaluated with respect to cell proliferation, ALP activity and osteocalcin production using MG-63 cells (human osteosarcoma cell lines) ${ }^{164,165}$. Glass fiber reinforced PEEK was found to enhance proliferation, ALP activity and osteocalcin production. PEEK with addition of $\beta$ TCP has been investigated with respect to bioactivity. However, the results showed no significant 
improvement for $\beta$ TCP on proliferation of osteoblast cells ${ }^{153}$. In addition, the cell viability and proliferation were even lower on $\beta$ TCP modified PEEK compared with pure PEEK. These results were confirmed by Petrovic et al. ${ }^{151}$, who studied PEEK containing 5, 10, 20 and 40 wt. \% $\beta$ TCP on human osteoblast cells. In contrast, a recent clinical study on $\beta$ TCP containing interbody cage for anterior cervical fusion showed promising results in a post-operative radiographic evaluation. The radiographic evaluation at the follow-up assessment after 3 and 12 months showed bone fusion in $70 \%$ and $95 \%$ of the patients, respectively ${ }^{166}$.

Recently, researchers have evaluated nano-sized HA-PEEK particles instead of conventionally sized ones to overcome the particle debonding from the matrix after long-term loading. Wang et al. ${ }^{167,168}$ examined the mechanical properties and microstructure of PEEK nanocomposite. The studies found no de-bonding occurring between the HA particles and PEEK matrix and the tensile strength reached a satisfactory level at $98 \mathrm{MPa}$ at $5.0 \mathrm{vol}$. HA \%. When the HA content exceeded 10 vol. \% a severe agglomeration of the particles occurred. These results can be compared to other studies that also found that the nanoparticles agglomerated during the fabrication and that it was difficult to distribute the particles evenly in the matrix. Ma and co-workers ${ }^{169}$ proposed a solution to the problem using an in situ synthesis process where the particles first were mixed into short-chained PEEK oligomers to achieve an increased bonding between the particles and matrix. The technique demonstrated optimal tensile strength at 6.1 vol. \% HA content.

Another reason for using nano-sized particles in biomaterial science is to mimic the nanometer size of the native cellular processes ${ }^{170,171}$. Some authors have found several unique features when altering the particle size from micro-to nanometer. Njuguna et al. ${ }^{172}$ demonstrated increased surface area to volume ratio and improved mechanical properties such as stiffness and tensile strength. However, the authors acknowledged that more research is needed to fully understand the material changes with nanoparticle reinforcement ${ }^{173}$. 
Nanostructured materials in general compared to conventional materials have been found to promote osteoblast adhesion, proliferation and differentiation to improve the bone growth ${ }^{174-176}$. Nanostructuring may therefore, be an interesting strategy for improving the bioactivity of PEEK. Hydroxyapatite nanorods incorporated into PEEK composite of different concentrations were investigated by $\mathrm{Li}$ et al. ${ }^{177}$ with respect to their mechanical, thermal and in vitro cell properties. At 21.6 and 29.2 vol. \% HA the tensile strength and fracture strain was in the region of human cortical bone. The higher concentration was found to promote osteoblast adhesion and proliferation compared to the other specimens. Nano sized $\mathrm{TiO}_{2} / \mathrm{PEEK}$ composites were prepared and evaluated against a PEEK polymer control $^{178}$. In addition, each group was fabricated with a smooth and a rough surface. The smooth surfaces were polished to a roughness average $\left(\mathrm{R}_{\mathrm{a}}\right)$ below $0.1 \mu \mathrm{m}$ and the rough surfaces were blasted by $\mathrm{TiO}_{2}$ particles to a $\mathrm{R}_{\mathrm{a}}$-value around 1.0-2.2 $\mu \mathrm{m}$. The results showed twice as much bone volume around the $\mathrm{TiO}_{2} / \mathrm{PEEK}$ implants compared to PEEK controls. Zhao and co-workers ${ }^{179}$ performed an in vitro quantitative proteomic analysis to evaluate the osteoblast response on nano-HA/PEEK composite compared to pure PEEK. The results showed significantly larger number of cells attached to the nano-HA/PEEK surface compared to that of pure PEEK. Several findings such as increased ALP activity and higher $\mathrm{Ca}^{2+}$ concentrations for nano-HA/PEEK may explain the increased adhesion and differentiation of osteoblasts to nano-HA/PEEK. A clinical study evaluating TLIF using two types of spine cages, nano-HA/polyamide66 cages (n-HA/PA66 cages) and pure PEEK cages was performed retrospectively ${ }^{180}$. One year after implantation the fusion and subsidence rates were evaluated using radiography. Results showed that both n-HA/PA66 cages and PEEK cages could provide comparable outcomes and are both effective materials for the TLIF procedure.

A combination of bioactive PEEK composite with nano-fluorohydroxyapatite (nano-FHA) and surface treatment compared with bare PEEK was compared in vitro and in vivo ${ }^{181}$. In vitro, the nanoFHA possess good antibacterial properties and induce osteogenesis. A co-effect was shown in vivo with a rough surface and incorporation of nano-FHA with improved bioactivity, osseointegration and 
bone-implant contact. The authors suggest this PEEK-based composite to be a possible modification for PEEK as a dental implant.

\section{Surface modification of PEEK}

Plasma treatment are used to etch, cross-link and activate polymers using different gas types ( $\mathrm{Ar}, \mathrm{He}$ or $\mathrm{N}_{2}$ ) and plasma conditions. With plasma treatment the wetting and frictions properties may be altered without changing the mechanical properties of the material ${ }^{182,183}$. Plasma treated PEEK surfaces using a microwave plasma in $\mathrm{NH}_{4} / \mathrm{Ar}$ and a downstream microwave plasma in $\mathrm{H}_{2} / \mathrm{Ar}$ were evaluated with respect to proliferation and differentiation of fibroblasts and osteoblasts $^{184}$. Using tissue culture polystyrene (TCPS) as a reference, the authors found a comparable osteogenic activity of cells on plasma treated PEEK. The method showed to be reproducible in stimulation and suppression of cell proliferation and successful in converting inert PEEK into a more bioactive biomaterial. Awaja et al. ${ }^{185,186}$ published two studies on plasma treated PEEK using a mixture of methane/oxygen $\left(\mathrm{CH}_{4} / \mathrm{O}_{2}\right)$ gas. The combination of $\mathrm{CH}_{4} / \mathrm{O}_{2}$ gas showed increased surface energy compared to untreated surfaces and those treated with $\mathrm{CH}_{4}$ alone. The cell adhesion and protein adsorption were favorable for plasma treated PEEK in $\mathrm{CH}_{4} / \mathrm{O}_{2}$ gas and could be correlated to the surface wettability.

A novel technique to improve the bioactivity of PEEK using accelerated neutral atom beam (ANAB) was employed by Khoury et al. ${ }^{187}$. ANAB uses an accelerated beam of Argon atoms to the surface and the treated surface shows alterations in the nano scale and increased surface hydrophilicity. Increased bioactivity was verified after the ANAB treatment with enhanced growth of seeded human cells on the surface. The results suggested ANAB treated PEEK to potentially increase the bioactivity by improving the speed and amount of bone formation.

As mentioned earlier, sulfuric acid $\left(\mathrm{H}_{2} \mathrm{SO}_{4}\right)$ is the only chemical agent that can dissolve PEEK. In addition, $\mathrm{H}_{2} \mathrm{SO}_{4}$ can be used to etch the surface and thereby change its topographical appearance. Zhao et al. ${ }^{188}$ created a $3 \mathrm{D}$ porous and nanostructured surface network using $\mathrm{H}_{2} \mathrm{SO}_{4}$ follow by water immersion and evaluated the surface 
topography, in vitro cell response and in vivo osseointegration (Figure 14). The results showed significantly improved bioactivity, cytocompatibility, osseointegration and bonding strength between the bone and PEEK material compared to the control. After the surface treatment the surface showed a $3 \mathrm{D}$ porous network with a distance between the pores of $0.5-1.0 \mu \mathrm{m}$ estimated on the SEM photographs. Additional rinsing in acetone was found to decrease the cytotoxicity caused by residual sulfuric acid on the surface. Similar finding were presented by another study where pure PEEK and carbon-PEEK was compared in vitro after having been treated with $\mathrm{H}_{2} \mathrm{SO}_{4}$ and $\mathrm{CaCl}_{2}$. The carbon-PEEK showed higher apatite-forming ability compared to pure PEEK. The authors hypothesized that the created surface provided functional groups for apatite formation and were incorporated into the dispersed carbon phase ${ }^{189}$. Several studies evaluating porous PEEK surface demonstrated similar findings as the previously mentioned investigation with additional data on improved antibacterial properties ${ }^{190,191}$.

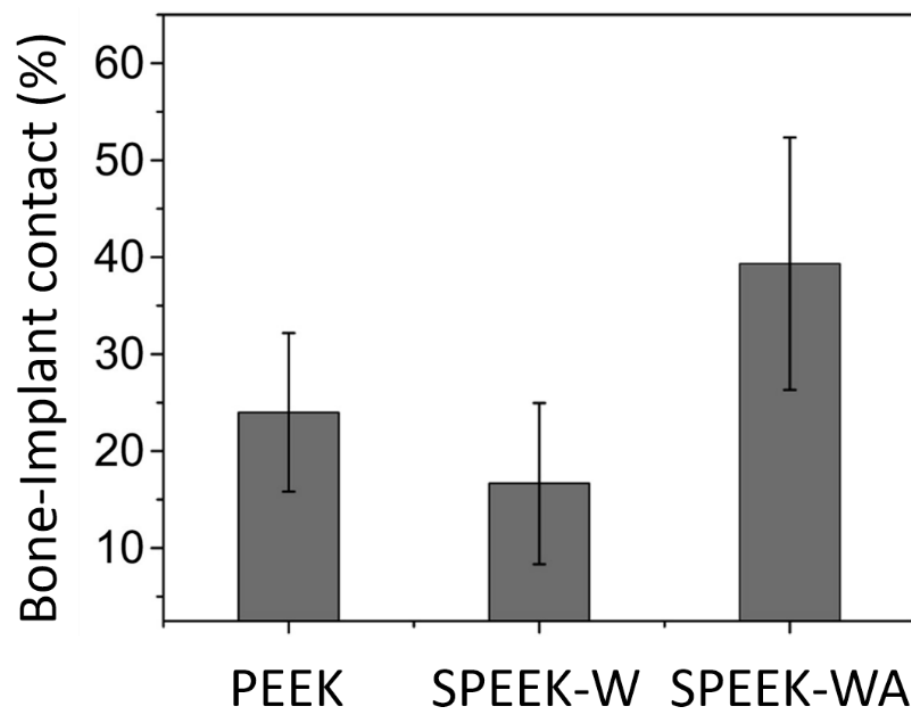

Figure 14. Bone-implant contact of neat PEEK, SPEEK-W (sulfonation treated PEEK and water immersion) and SPEEK-WA (sulfonation treated PEEK and acetone immersion) after 8 weeks of healing. Figure from Zhao et al. ${ }^{\mathrm{VIII}}$ 
The last and the most frequently evaluated way to enhance the bioactivity of PEEK is to deposit a bioactive material on the surface. Over the years many different coating materials has been assessed such as HA, titanium, gold, carbon and tert-butoxide.

Hydroxyapatite is by far the most common coating material because of its stoichiometric ratio close to that of human bone ${ }^{192}$. As for the bioactive composite using HA, several studies have proven the excellent biocompatibility and osteoinduction in vivo of hydroxyapatite ${ }^{136-139}$. Since PEEK is fairly porous and sensitive to temperatures above the melting point the applied coating technique must be carefully selected. Cold spray method to generate HA coating on PEEK was evaluated by Lee et al. ${ }^{193}$ with respect to the bioactivity in vitro and in vivo. PEEK cylinders were coated and placed in a rabbit ilium model and uncoated samples were used as controls. The results showed a homogenous layer of HA coating and the bonding to the substrate was durable without any structural deformations of the core material. The in vivo tests of the HA-coated cylinders demonstrated a promoted osseointegration on the micro-CT examination. Hydroxyapatite was deposited on PEEK using aerosol deposition and the final result showed no pores and strong adhesion to the substrate ${ }^{194}$. The in vitro and in vivo results showed clear improvement in biocompatibility in terms of cell adhesion, proliferation, differentiation and BIC. Rabiei et al. ${ }^{195}$ increased the bioactivity of PEEK by three steps of surface treatment. Firstly, the samples were exposed to oxygen plasma in order to alter the surface chemistry to increase the bonding sites. Secondly, the coating deposition was carried out using a RF magnetron sputtering system. The coating composition was a dual-layer of yttria-stabilized zirconia (YSZ) covered by an HA layer. The final step was a heat treatment to mimic the clinical reality using a steam autoclave. Oxygen plasma treatment removed the hydrocarbon molecules and increased the wettability. In general the HA/YZS coating was found to improve the initial cell attachment and proliferation of osteoblast cells. PEEK composite with 30 vol. \% magnesium $(\mathrm{Mg})$ coated with an HA layer by treatment in an aqueous solution was evaluated by Jung and colleagues ${ }^{196}$. The coating was found to inhibit bio-corrosion and improve bioactivity compared to pure PEEK and uncoated PEEK/Mg 
samples in vitro using MC3T3-E1 cells. Barkarmo et al. ${ }^{197}$ manufactured PEEK implants and coated them with HA using the same technique as for this study and compared them with uncoated samples. The quality of the coating was analyzed using SEM, AFM and XPS and the osteoinductive properties were quantified with histomorphometry. Despite some problems with primary stability the results showed higher BIC for the HA-PEEK, however without statistical significance. A recent study by Ozeki et al. ${ }^{198}$ created thin HA films on titanium (Ti) coated PEEK specimens using a sputtering process. The intermediate layer of Ti was applied with different thickness to evaluate the adhesion of the HA coating. The results demonstrated a successfully formed HA film and intermediate layers of $\mathrm{Ti}<10 \mathrm{~nm}$ showed higher adhesion strength than the ones of $>50 \mathrm{~nm}$. Intermediate Ti layers $<5 \mathrm{~nm}$ were found to release more Ti ions compared to the samples with thicker layers.

Since the introduction of titanium implants, they have remained the commonly used material in the oral cavity due to its biological properties $^{199}$. Titanium can also be used as a coating material for PEEK. An in vitro study by Yao and co-workers evaluated osteoblast adhesion on PEEK coated with titanium or gold using plasma deposi$\operatorname{tion}^{200}$. The osteoblasts were more attached and spread out on the coated PEEK compared to uncoated version. An early study from 1995 by Cook et al. ${ }^{201}$ installed PEEK implants into femur of mongrel dogs with PEEK implants coated with titanium using plasma vapor process. Removal torque values were significantly higher for uncoated implants at 4 weeks but there were no differences at 8 weeks. However, the coated PEEK implants had significantly higher percent of BIC compared to the uncoated implants. Dense and uniform titanium can laminate PEEK using electron beam deposition at low temperatures ${ }^{202}$ (Figure 15). In vitro results showed doubled level of proliferation and differentiation of cells (MC3T3-E1) around titanium coated PEEK cf. uncoated versions. Moreover, the BIC was significantly higher around Ti-PEEK compared to pure PEEK. Another study by $\mathrm{Ha}$ et al. ${ }^{203}$ evaluated $\mathrm{NaOH}$ treated CFRPEEK coated with titanium with vacuum-plasma-spraying (VPS). Evaluation was performed in SBF with similar ion concentration as the human blood. Observations using SEM, energy-dispersive X-ray 
(EDX) and diffuse reflectance Fourier transformed-infrared spectroscopy showed a calcium phosphate layer on the $\mathrm{NaOH}-\mathrm{PEEK}$. On the untreated samples there were no calcium phosphate on the surfaces. The authors concluded that $\mathrm{NaOH}$ impregnation after coating CFR-PEEK with titanium is a successful method to improve the bioactivity for bone apposition on CFR-PEEK. An in vivo study by Walsh and co-workers ${ }^{204}$ compared the mechanical and histological properties of Ti-PEEK and PEEK at the bone interface. Implant pins were placed into the tibia and femur of adult sheep with a pressfit approach. After 4 and 12 weeks of healing the implants were either pushed out or submitted to histomorphometry. The shear strength was higher with the titanium coating compared to uncoated PEEK. In addition, direct bone ongrowth was found on the titanium coated samples after both healing periods.

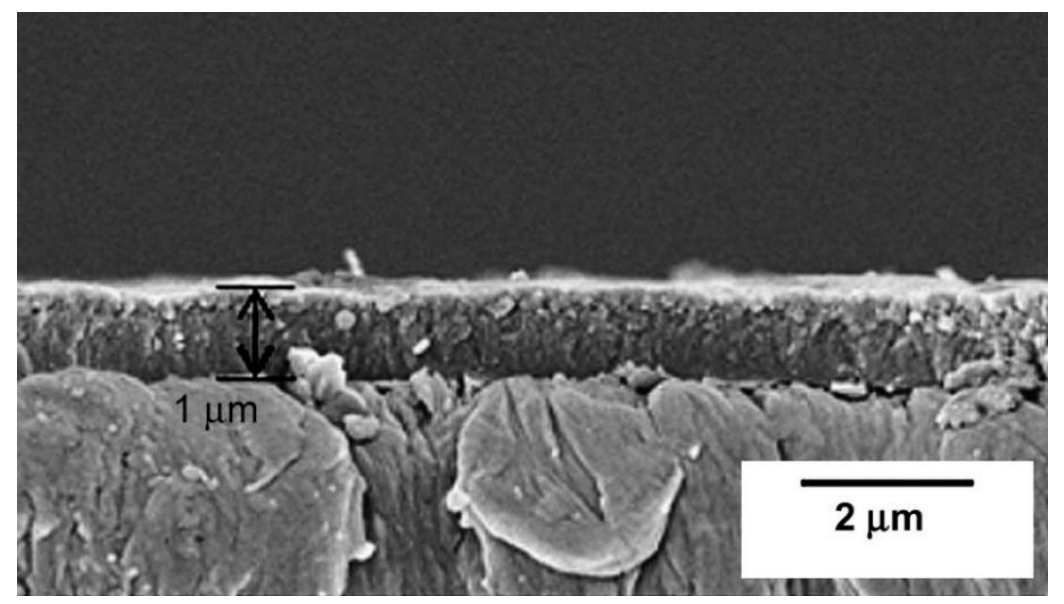

Figure 15. PEEK OPTIMA disc coated with titanium using electron beam deposition. Image displayed in cross-section. Figure adapted from Han et al. ${ }^{\text {IX }}$

Titanium is naturally covered by different types of oxides of which titanium dioxide $\left(\mathrm{TiO}_{2}\right)$ is dominant and is a material shown to possess good bioactive properties. Titanium dioxide is generally harvested from three different mineral sources; ilmenite, rutile and anatase. The anatase and rutile phase can be deposited onto PEEK to increase its bioactive appearance. Few authors have evaluated PEEK 
coated with $\mathrm{TiO}_{2}$ in vivo, have investigated the osteoblast response to $\mathrm{TiO}_{2}$ on PEEK. Tsou et al. ${ }^{205}$ applied a thin film of $\mathrm{TiO}_{2}$ onto PEEK using an arc ionplating technique at a low temperature. The results showed better osteoblast compatibility for $\mathrm{TiO}_{2}$ coated PEEK compared to untreated PEEK. In addition, the osteogenesis properties were improved by means of increased levels of osteopontin, osteocalcin and other calcium containing components. Rutile $\mathrm{TiO}_{2}$ was also found to perform better than anatase $\mathrm{TiO}_{2}$. A study using the same technique of $\mathrm{TiO}_{2}$ application as the previous investigation was conducted by Chi et al. ${ }^{206}$ and found comparable results in an in vivo model. In vivo studies of PEEK and $\mathrm{TiO}_{2}$ have evaluated similar properties; removal torque, micro-CT and histology. All available studies showed improved performance on all measured properties and the most prominent results was shown using the rutile $\mathrm{TiO}_{2}$. Results further showed that a rougher implant surface improved the osteoconductivity ${ }^{178,207-209}$. 


\section{AIMS}

The general aim of this thesis was to improve the biocompatibility of a PEEK polymer by altering the surface topography and chemistry to increase its applications in the dental and orthopedic field.

Specific aims of this thesis were:

1. To analyze the mechanical properties before and after a coating procedure, characterize the surface topography and chemistry on a neat and an HA-coated PEEK surface (Study I-III).

2. To evaluate the adhesion of HA-coating on the PEEK implant after the friction caused by the implant in a biomedical test block (Study II).

3. To in vivo investigate the biomechanical properties after 3 , 12 and 20 weeks of healing by measuring the amount of removal torque required to unscrew a nano HA-coated PEEK implant. The results are compared with an unmodified PEEK implant (Study I and IV).

4. To assess the in vivo bone response to HA-coating in terms of bone-implant interactions and to evaluate the osteoinductive properties of PEEK implant with an apical perforation (Study II, III and IV). 


\section{MATERIALS AND METHODS}

\section{Implant and Coating Preparation}

The PEEK implants (Invibio Ltd, Lancashire, UK) were milled and designed with a non-cutting apical shape to minimize mechanical stress at insertion rotation. The implants were $3.5 \mathrm{~mm}$ in diameter and $4 \mathrm{~mm}$ long (Figure 16A). The implants for evaluation in the femur bone was designed with a hole penetrating the apical part of the implant (Figure 16B). The hole was created to make it possible to evaluate bone ingrowth in an area only surrounded the PEEK polymer thus evaluating the osteoinductive properties of PEEK and HA. This inside surface of the hole is not affected by the insertion friction so that the coating is fully intact in situ. This design makes it similar to the clinical reality as a spinal implant.

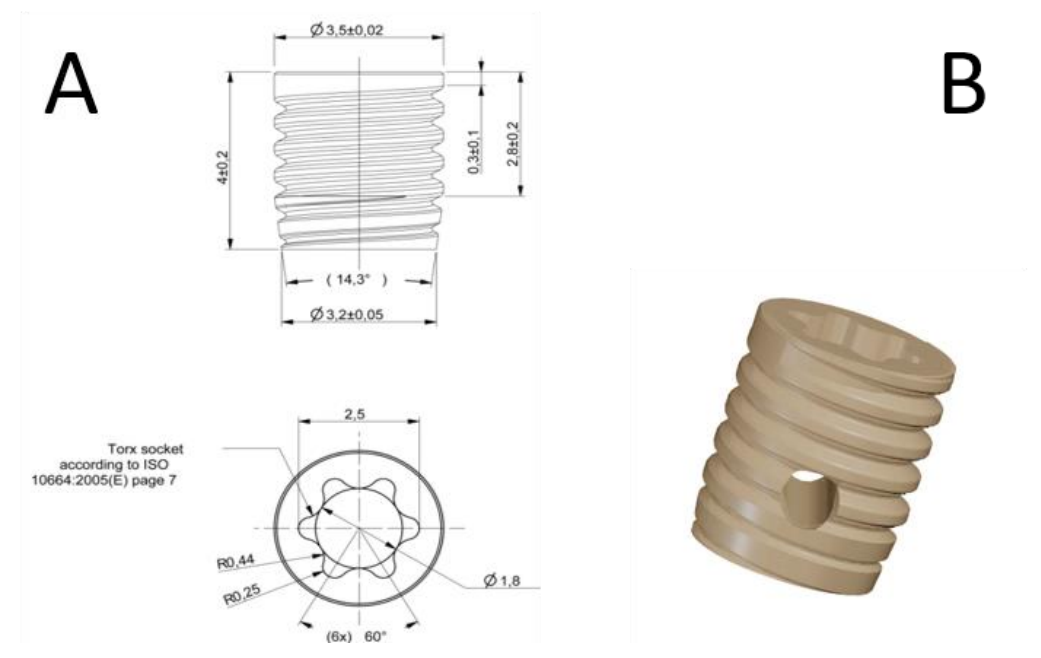

Figure 16. Technical drawing of PEEK implant (A) and 3D rendered image of the implant with an apical perforation to enable evaluation of the bone fusion into a healing chamber (B). 
The coating compound is a synthetic nano-sized crystalline calcium phosphate with an average particle size of 1-10 nm. The crystals are rod-shaped with an average diameter of 2-10 nm and a length of 20$50 \mathrm{~nm}$ which can be estimated from TEM analyses (Figure 17).

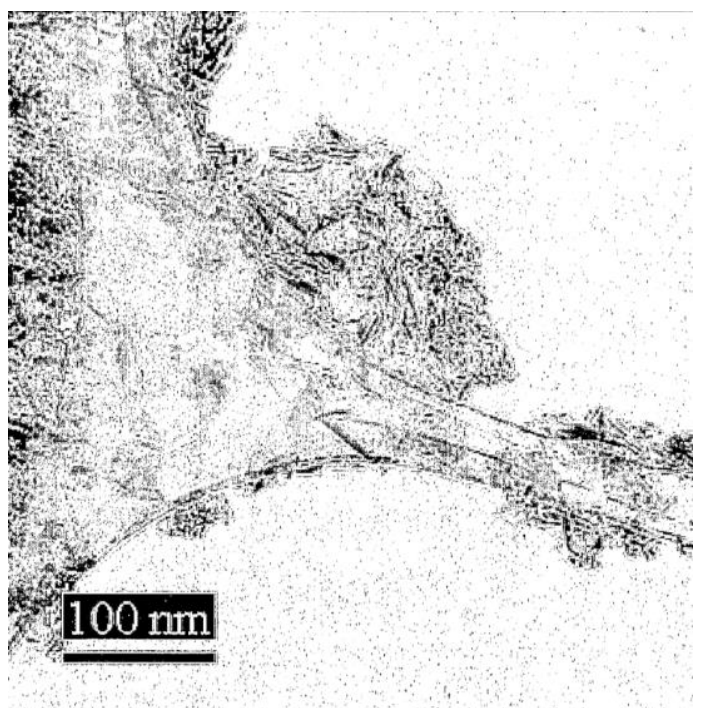

Figure 17. Transmission election microscope (TEM) of HA crystals in Promimic AB invention. Figure from patent description by Kjellin and Andersson. ${ }^{\mathrm{X}}$

Detailed description of the implant coating procedure ${ }^{210}$ (Figure 18):

1. As per stoichiometry, phosphorus and calcium salts are dissolved in water.

2. To create a liquid crystalline phase, surfactant as well as hydrophobic organic solvent is added and allowing the liquid crystalline phase to equilibrate.

3. The liquid is placed in an ammonia atmosphere to raise the $\mathrm{pH}$ so that the crystals of calcium phosphate are formed in water domains of the liquid crystalline phase. All previous steps are performed in room temperature.

4. The micro-emulsion is applied onto the implant surface and the implant is rotated at $2700 \mathrm{rpm}$ for 5 seconds to distribute 
the coating on the surface.

5. A heat treatment $\left(325^{\circ} \mathrm{C}\right.$ in oxygen-enriched atmosphere) for 5 minutes removes the surfactants and bonds the coating to the implant surface. The final thickness of the coating is 20$40 \mathrm{~nm}$.

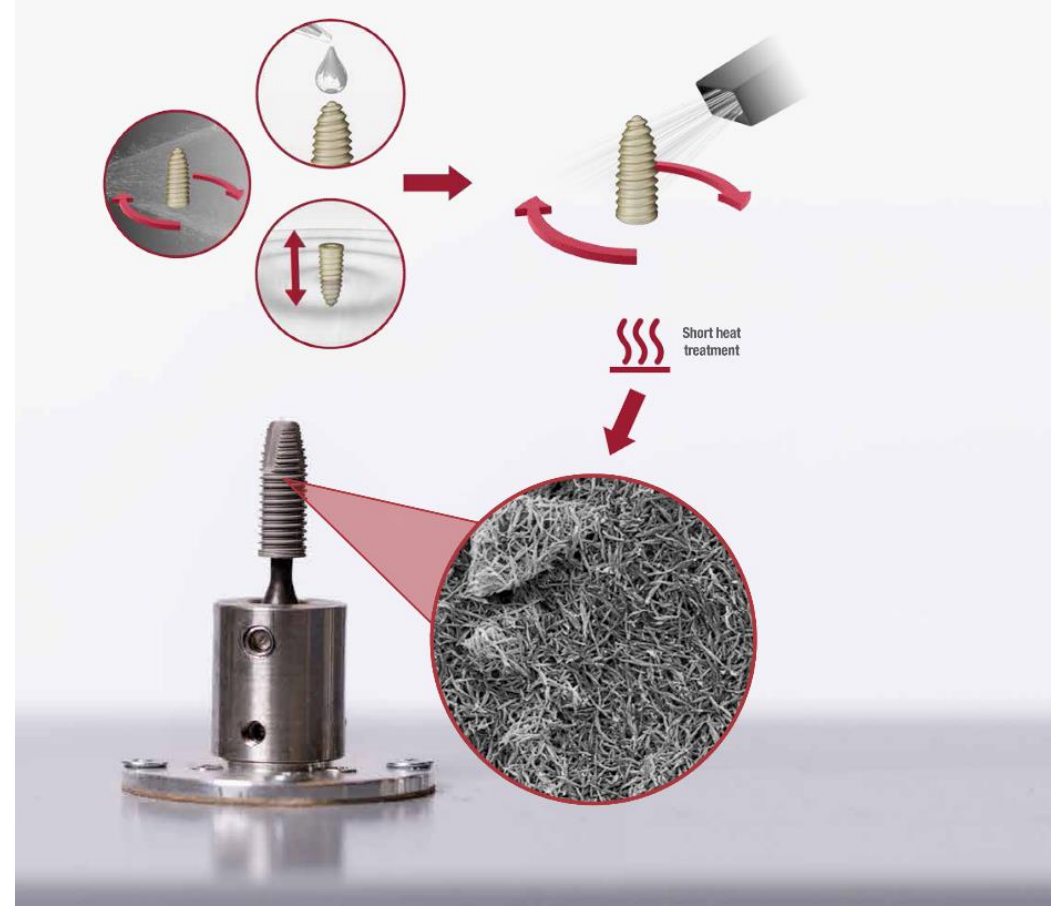

Figure 18. Illustrative description of the coating procedure in this study. The coating is based on a wet chemical approach where the coating is homogenously distributed over the surface, regardless of shape.

\section{Surface Characterization}

\section{Optical interferometry}

Interferometry is a device using waves, usually light, to extract topographical data at a micrometer level and submicron-meter from a surface. In short, one wave is split into two waves. One is reflected 
from a reference mirror and the other one from the sample surface. After these two waves are recombined to interfere, the imaging lens can create the interferogram onto the CCD camera.

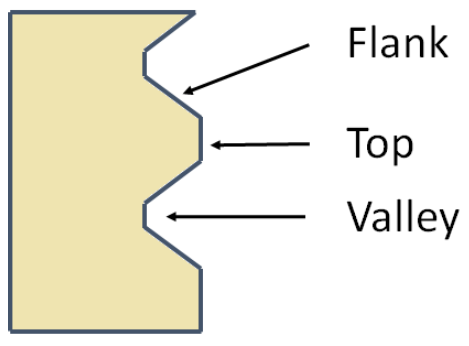

Figure 19. Description of points of measure using the optical interferometer. Three measurements per site on three discs was performed.

In this thesis the surface topography of the neat and HA coated PEEK implant were characterized and compared using a MicroXAM instrument (ADE Phase Shift, Tucson, AZ, USA). The surface topography was presented in the micrometer level in Study I. With X50 objective and zoom factor of 0.625 , the disc surface area ( 200 X 260 $\mu \mathrm{m})$ was investigated. To remove errors of form and waviness a Gaussian filter of 50 X $50 \mu \mathrm{m}$ was applied. Each implant was scanned in random areas of 3 thread tops, 3 flanks and 3 valleys (Figure 19). Three samples from each group were measured $(n=6)$ according to guidelines by Wennerberg and Albrektsson ${ }^{211}$. Three dimensional renderings of the surfaces were created using Surface Scan Software (Somitronic Instrument, Lyon, France). The following topographical parameters were used to describe the surface topography:

- $\mathrm{S}_{\mathrm{a}}$ : the average deviation in height in relation to a prenominal plane $(\mu \mathrm{m})$

- $S_{\mathrm{ds}}$ : density of summits, a spatial parameter $\left(\mu \mathrm{m}^{-2}\right)$

- $S_{\mathrm{dr}}$ : the developed surface ratio to a flat surface $(\%)$

Atomic force microscopy

Atomic force microscopy (AFM) is a type of scanning probe microscopy that creates nanoscale images of natural surfaces (without prior 
need of surface modification) using a scanning probe (Figure 20). When the probe is moved over the sample, a laser beam reflects the movement from the reverse side of the cantilever to a position-sensitive detector. The output data is transferred into $\mathrm{x}, \mathrm{y}$ and $\mathrm{z}$ coordinates by a controller and further sent to the computer for data acquisition, display and analysis ${ }^{212}$.
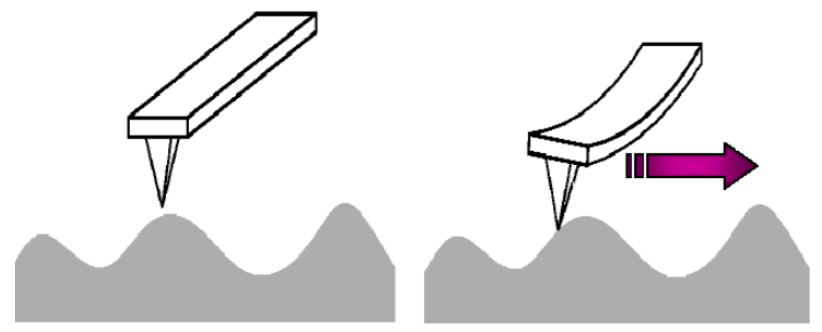

Figure 20. Atomic force microscopy (AFM) is an analysis technique of a rigid surface down to the nano-meter level. AFM uses a probe in contact or non-contact mode to scan and create images of the surface.

Atomic force microscopy was used in this thesis as a complement to interferometry to further investigate the PEEK and HA-PEEK surface topography but at the nanoscale. An AFM microscope from Parks System (XE-100, Suwon, South Korea) was used and the results was published in Study I. The scanning was performed on discs $(\varnothing 10 \mathrm{~mm})$ in air and at room temperature. Three discs of PEEK and HA-PEEK were analyzed on three randomly selected positions $(n=6)$. Measurements were performed in a non-contact mode with two scan areas, $1 \times 1 \mu \mathrm{m}^{2}$ and $10 \times 10 \mu \mathrm{m}^{2}$. After leveling by subtraction, a Gaussian filter was applied $(0.25 \times 0.25 \mu \mathrm{m}$ and $2.5 \times 2.5$ $\mu \mathrm{m}$, respectively. Graphic 3D image renderings were processed with the imaging software Mountain Map (Digital Surf, Besançon, France). The topographical parameters $\left(S_{a}, S_{d s}, S_{d r}\right)$ were selected to be combined with the results from the interferometer.

\section{Scanning electron microscopy}

Scanning electron microscopy (SEM) is a device that creates images of an object with a resolution better than 1 nanometer using electrons instead of light. The object is scanned with a focused beam of 
electrons that infiltrates the outer surface layer and transfer energy to electrons inside the sample. Electrons are backscattered from the sample to a detector that creates an image of the surface. All nonmetal samples need to be covered by a thin layer of conductive material, such as gold. This preparation is called sputter coating.

SEM was performed in study I, II and III with different angles and locations of the PEEK implant to visualize the surface topography at a nanoscale level. The surface morphology was performed using a SEM from ZEISS (LEO Ultra 55 FEG, Oberkochen, Germany) at an accelerating voltage of $2-4 \mathrm{kV}$ (Figure 21). To make the surface conductive, the implants were sputtered with gold, using a JEOL JFC$1100 \mathrm{E}$ (JEOL Ltd, Tokyo, Japan) operating at $10 \mathrm{kV}$ for 90 seconds.

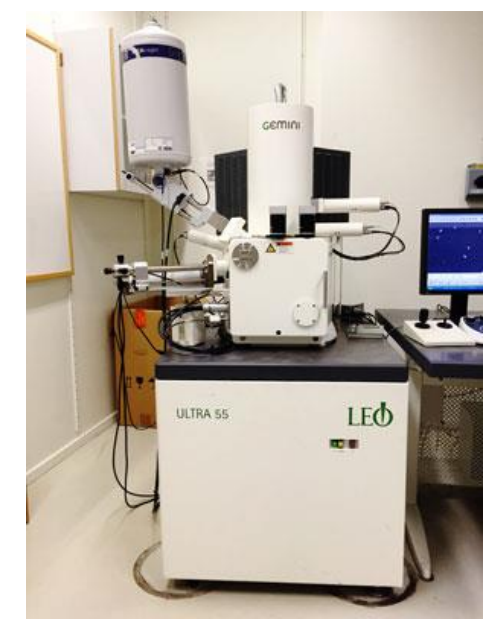

Figure 21. The LEO Ultra 55 is a scanning electron microscopy with a resolution down to $1 \mathrm{~nm}$.

\section{Contact angle measurements}

The contact angle of liquid on a surface is an established method to quantify the surface energy and wettability that is related to the surface roughness ${ }^{213}$. When the contact angle is between $0^{\circ}$ and $90^{\circ}$, the surface is considered to possess good wetting abilities. However, if the angle is above $90^{\circ}$ the surface is not wettable. A non-wetting surface is in other words hydrophobic since it repels water.

A contact angle measurement was performed in Study I to compare the surface energy before and after the coating procedure. The 
measurement was performed with a DAT 1100 from FIBRO System (Hägersten, Sweden). Water (Type 1, 18.2 M $\Omega$ was used as a test liquid and the drop volume was $4 \mu \mathrm{l}$. The measurements were done on PEEK discs since contact angle measurements on threaded surfaces are difficult to interpret.

\section{Chemical and Mechanical Characterization}

\section{X-ray photon spectroscopy}

$\mathrm{X}$-ray photoelectron spectroscopy (XPS) is an analytical technique to identify and quantify the chemical elements on the outermost layer of a surface. XPS uses an X-ray beam to irradiate the sample and quantify the kinetic energy and number of electrons that are projected from the material. With this technique we are able to obtain the chemical composition of various materials.

In study I the chemical assessment of the surface was performed with an XPS (PHI 5000C ESCA System; PerkinElmer Inc., Waltham, MA, USA). Spectra were obtained at an operating angle of $45^{\circ}$, at $200 \mathrm{~W}$ with an $\mathrm{Al} \mathrm{K}$-alpha excitation source. Two locations on each sample were analyzed; at the top and bottom of the implant screw. The mean values were calculated and compared.

\section{Coating adhesion in Sawbones $\AA$ simulation model}

Concerns whether the coating persists on the implant surface after insertion friction encouraged a coating stability test in biomedical test blocks (Sawbones ${ }^{\circledR} 40$ PCF; Pacific Research Laboratories, Washington, DC). Sawbones provides a uniform and consistent artificial bone with eliminates the natural variations in authentic bone. The blocks consists of polyurethane foam in this study with a density resembling natural bone.

Three coated and three uncoated implants were used for the measurements. The insertion and removal torque of the implants was monitored with a torque gauge (Tohnichi BTG90CN, America Corp, Buffalo Grove, IL, USA). SEM analysis revealed residues of Sawbones debris on the surface after removal. These residues were removed by sonicating each implant in $15 \mathrm{~mL}$ ethylene diamine (purum, $\geq 99.0 \%$, Fluka) for $15 \mathrm{~min}$, using an ultrasonic cleaner (VWR USC100T, VWR, Leuven, Belgium). The implants were analyzed 
with a scanning electron microscope (SEM, LEO Ultra 55, Zeiss, Oberkochen, Germany) prior to and after the insertion testing. The results are presented in study I.

\section{Tensile testing}

The strength of a material under tension is a very important physical characteristic in the field of biomaterials and especially for load bearing implants. The process involves placing the specimen in the apparatus where a force is applied slowly until it fractures. The applied force against the elongation is recorded and presented in a strain-stress diagram. Since heat treatment was involved in the coating procedure of the PEEK implants, it was of interest to evaluate how the heat treatment affected the mechanical strength of the PEEK material.

In study I, the mechanical testing was performed in accordance with the tensile testing standard, the International Organization for Standardization (ISO) 527-2. Samples for the testing were provided by Invibio Ltd. The specimens were of type 1A in the ISO 527 standard, with a total length of $200 \mathrm{~mm}$, a thickness of $4 \mathrm{~mm}$, and a midsection width of $10 \mathrm{~mm}$. The samples were heat-treated at $300^{\circ} \mathrm{C}$ and $350^{\circ} \mathrm{C}$ for 10 minutes, i.e., a time twice as long as used when applying the HA coating. Five samples were tested at each temperature. The measurements were performed by Swerea IVF AB (Mölndal, Sweden). An MTS 20/M (MTS Systems Corporation, Eden Prairie, MN, USA) was used for each measurement.

\section{Experimental Animal Model}

\section{Animals and ethical approvals}

The experimental procedures were performed in two different animal facilities, one in Sweden and another in France. The rabbits were adult, mixed gender and they weighed approximately $3.3-4.2 \mathrm{~kg}$. Compared to humans, rabbits have a different bone microstructure and compositional properties. However, there are some similarities in bone mineral density and fracture properties ${ }^{214}$. One major difference is the bone remodeling and speed of skeletal change ${ }^{215}$. Rabbits have about 3-4 times faster remodeling period compared to humans $^{216,217}$. Therefore, the experimental protocol and healing time 
points are established in accordance with these physical characteristics.

The animal study in Sweden was approved by the Malmö/Lund regional animal ethics committee and all experiments were performed following the institutional and national guidelines and regulations of the Board of Agriculture (Dossier number: M17-12). In France, the experiments were done at the Center of Biomedical Research of the École nationale vétérinaire d'Alfort, Alfort, Paris, and the study was approved by the National Committee of Ethical Reflection on the Animal Experimentation (Comité National de Réflexion Ethique sur l'Expérimentation Animale) and from the French Ministry of Higher Education and Research (Ministère de l'Enseignement Supériur et de la Recherche) with dossier number: 13-011 and 00391-01.

\section{Anesthesia}

In the Swedish animal facility, on the day of surgery after fasting, the animals were anesthetized by a mixed injection consisting of 0.15 $\mathrm{mL} / \mathrm{kg}$ medetomidine ( $1 \mathrm{mg} / \mathrm{mL}$ Dormitor ${ }^{\circledR}$; Orion Pharma AB, Sollentuna, Sweden) and $0.35 \mathrm{~mL} / \mathrm{kg}$ ketamine hydrochloride $(50$ $\mathrm{mg} / \mathrm{mL}$ Ketalar®; Pfizer AB, Sollentuna, Sweden).

In the French animal facility the surgery was performed under general anesthetic using a dose of $250 \mathrm{ll} / \mathrm{kg}$ of medetomidine (Domitor, Zoetis, France), $20 \mathrm{mg} / \mathrm{kg}$ of ketamine (Imalgene 1000, Merial, Sanofi, France) and $1 \mathrm{mg} / \mathrm{kg}$ of diazepam (Valium, Roche, France).

\section{Surgical procedure and implantation}

The surgical procedure was identical for all studies and irrespective of the animal facility and rabbit strain. To avoid contamination the hind limbs were shaved and washed with $70 \%$ ethanol (Solveco AB, Rosersberg, Sweden) and $5 \mathrm{mg} / \mathrm{mL}$ chlorhexidine (Fresenius Kabi $\mathrm{AB}, \mathrm{Bad}$ Homburg, Germany). After injection of approximately 1 $\mathrm{mL}$ local anesthesia (Xylocaine; AstraZeneca AB, Södertälje, Sweden), a full thickness incision on the skin, muscles and periosteum was made and the medio-proximal tibial bone was exposed. In study III and IV the incision was extended to permit installation of implants in the proximal femoral region. For both tibia and femur implantation, the bone was trephined under constant irrigation (sterile 
saline) with a series of drills up to a final diameter of $3.2 \mathrm{~mm}$. Tapping was performed manually before the implant was installed by hand. The implants were submerged into the bone with the implant shoulder in line with the bone level. The wound was cleaned and generously irrigated with sterile saline before the fascia and skin was closed separately with bioresorbable sutures (Ethicon, Norderstedt, Germany).

\section{Experimental model}

The tibia implants were divided into two groups; PEEK (control) and HA-PEEK (test). Each rabbit receives three PEEK and three HAPEEK implants randomized allocated on the left and right hind limb, but never on the same side (Figure 22). Implants inserted in the femur were designed with an apical perforation. This experimental model allows a pair-wise comparison within the same animal which has a higher degree of statistical power than only comparing the means.

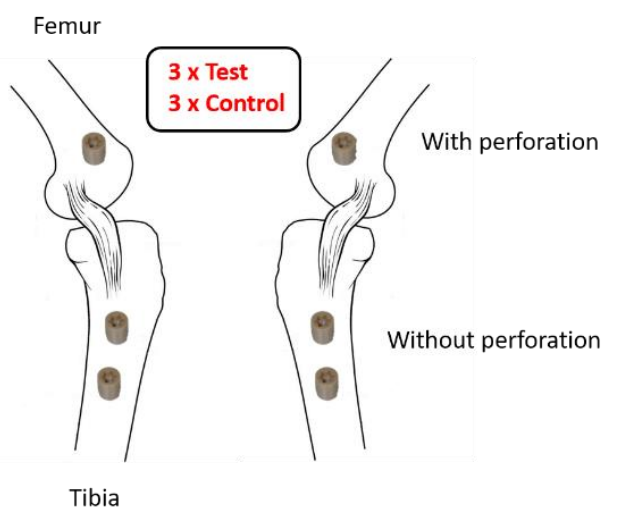

Figure 22. Schematic description of implant location in femur and tibia. Implants in femur are designed with a perforation and the implants in the tibia are without perforation.

The evaluation time from insertion to euthanasia was 3,12 and 20 weeks. Study I-III assesses 3 and 12 weeks of healing and study IV evaluates an extended healing time of 20 weeks. Three weeks of healing refers to the early stage of bone formation and after 12 weeks a more complete bone formation is expected ${ }^{215,218}$. After the 
results from 3 and 12 weeks were assessed a decision was made to add an extended healing time to further understand the effect of the HA-coating.

\section{Post-operative care and euthanasia}

The first 24 hours the rabbits were carefully evaluated, checking for signs of pain and distress. In the Swedish animal facility, postsurgical analgesic buprenorphine hydrochloride $(0.5 \mathrm{~mL}$ Temgesic; Reckitt Benckiser, Slough, UK) was administrated for 3 days. In the animal facility in France, for five days after surgery, the animals were administrated analgesic therapy through injection of buprenorphine (Buprecare, Animalcare, UK) and meloxicam (Metacam; Boehringer Ingelheim Vetmedica, Inc., St Joseph, MO, USA). To further reduce the pain, a fentanyl patch (Duragesic, Janssen Pharmaceutica, Beerse, Belgium) was applied onto the skin for three days. Antibiotics (Enrofloxacine, Baytril, Bayer Animal Health, Germany) was also administrated through the drinking water $(200 \mathrm{mg} / \mathrm{l})$.

The animals were kept in single housing cages with space enough to move freely and carry out their normal behavior. After each healing period the euthanization was carried out using an overdose of sodium pentobarbital $(60 \mathrm{mg} / \mathrm{mL}$; Apoteksbolaget AB, Stockholm, Sweden).

\section{Ex vivo analysis}

\section{Removal torque testing}

A common method to evaluate the level of osseointegration is to record the highest required removal torque (RTQ) to detach the bonding between bone and the implant. Removal torque testing is a simple method but with a very complex biomechanics. There are several parameters that affects the RTQ values; surface roughness, thread design, anatomy and density of surrounding bone etc. How they interplay and to what extent is today still not fully understood $^{219}$.

Immediately after euthanasia the tibia and femur were dissected and the soft tissue was removed to allow access to the implant. The implant in the distal tibia was coupled to a calibrated digital torque 
meter (Tohnichi, Tokyo, Japan) and the highest removal torque (RTQ) was recorded in $\mathrm{Ncm}$.

\section{Gene expression analysis}

Investigations to further understand the underlying events linked to a successful treatment, the cellular and molecular responses often need to be assessed. Gene expression is a process of how a gene is used to affect an organism's traits by internal and external stimuli. Gene expression could for instance alter the cell phenotype or recruit more cells of a certain phenotype. Investigating the expressed genes may provide information on the phenotype of the cells which could explain the treatment outcome. Osseointegration of a biomaterial is dependent by the balance between osteoclasts and osteoblasts. Osteoblast, osteoclast and other inflammatory cells express specific marker genes that have different impact on the healing event. By quantifying the regulation of these expressed genes one can further understand the cellular response of the treatment.

In this thesis, the gene expression was investigated using a real-time quantitative polymerase chain reaction (qPCR). With qPCR the RNA is reversely transcribed into complementary DNA (cDNA) to detect and quantitate gene expression products. The reactions is monitored in "real time" during the PCR and the regulation of the specific gene is compared to a reference gene.

\section{Sample collection and protection}

Remnants of cell and tissue around the implants were collected from the removed implants after the RTQ procedure. Each implant was submerged in RNAlater (LifeTechnologies, Thermo Fisher Scientific, Waltham, MA, USA) solution at room temperature. The solution permeates the cells and stabilize and protect cellular the ribonucleic acid (RNA). The samples were stored at $-20^{\circ} \mathrm{C}$ until further processing.

\section{$R N A$ preparation and quantification}

To access the mRNA inside the cells without degrading the RNA by different enzymes, the cells need to be disrupted and purified. First, the tissue and cells from the explanted implants were disrupted with 
an ultrasonic processor (Sonoplus, Bandelin, Germany) and PUREzol ${ }^{\circledR}$ reagent (BioRad, Hercules, CA, USA) and then treated with chloroform. To extract the RNA, PUREzol ${ }^{\circledR}$ reagent (BioRad, Hercules, CA, USA), Aurum Total RNA Fatty and Fibrous tissue (BioRad, Hercules, CA, USA) were used. These agents lyses cells and tissues, deproteinates RNA, and inactivates enzymes (RNase) from a variety of sources (cells, fatty tissue and fibrous tissue). The collected RNA was eluted with nuclease-free water.

The amount of extracted RNA was quantified with NanoDrop ND-1000 Spectrometer (ThermoScientific, NanoDrop Technologies, USA) at an optical density of $260 \mathrm{~nm}$.

\section{Pre-amplification and synthesis of $c D N A$}

The first attempt to extract RNA indicated too low values for processing the prepared markers. Therefore, a pre-amplification was required to obtain sufficient amount of cDNA. Via reverse transcription, cDNA was synthesized from RNA template using an enzyme (reverse transcriptase). To perform this, RNA yields from the samples, the cDNA was pre-amplified before the gene expression and Sso Advanced PreAmp Supermix (BioRad, Hercules, CA, USA) was used. 
Real-time polymerase chain reaction ( $q P C R$ )

Real-time PCR is a process where each sample is illuminated with specific wavelength to detect the intensity of the emitted fluorescence. The process runs in multiple cycles with different temperatures where the enzymes are functioning. Throughout the cycles the genes are multiplied until billions of copies are created. After a certain amount of cycles (usually around 40) most of the genes have been emitted detectable fluorescence (Figure 23). In this thesis we used SYBRGreen ${ }^{\circledR}$ (BioRad, Hercules, CA, USA) to bind to the genes and alter the structure to enhance fluorescence.

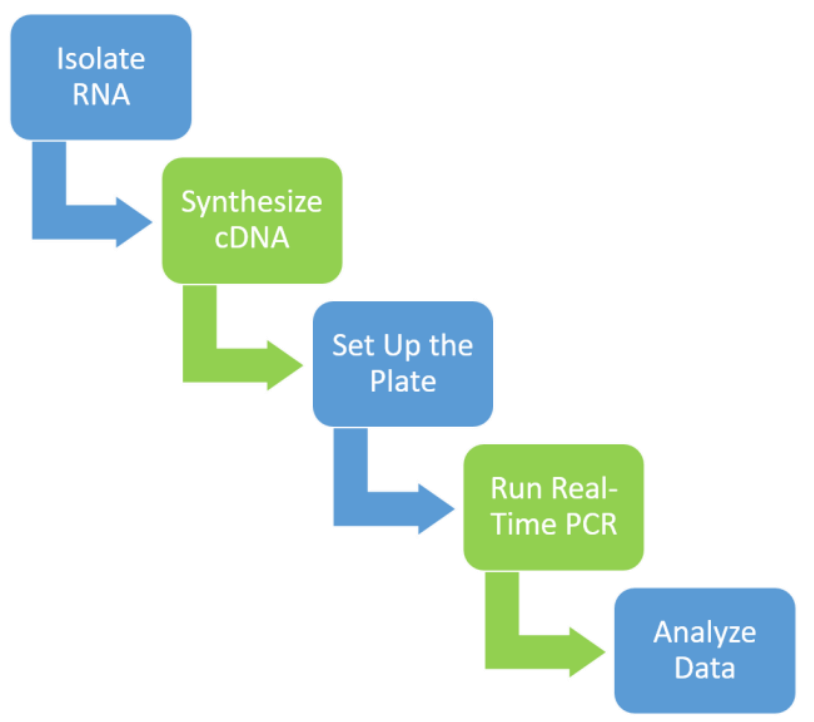

Figure 23. Workflow of RT-PCR.

The normalized relative quantification method $(\Delta \Delta \mathrm{Cq})$ was used where the changes in gene expression in a given sample is analyzed relative to a reference gene. In this thesis we selected the following genes that are involved in osteogenesis; BGLAP, BMP2, COL1A1, IGF1, RUNX2, SSP1. The reference genes to relate the expression to; GAPDH, ACTB and LDHA (Table 4). 
Table 4. List of genes with description included in this thesis.

\begin{tabular}{|c|c|c|}
\hline Gene & Symbol & Description \\
\hline Bone gamma-carboxyglutamate (gla) protein & BGLAP & $\begin{array}{l}\text { Osteocalcin. Encodes abundant bone protein } \\
\text { secreted by osteoblasts to regulate bone remodelling } \\
\text { and energy metabolism. }\end{array}$ \\
\hline Bone morphogenetic protein 2 & BMP2 & $\begin{array}{l}\text { Encodes a secreted ligand of TGF-beta which recruits } \\
\text { and activate transciptions factor which plays a role in } \\
\text { bone and cartilage development. }\end{array}$ \\
\hline Collage type I, alpha 1 & COL1A1 & $\begin{array}{l}\text { Encodes type I collagen found in connective tissue } \\
\text { and rich in bone and cartilage. }\end{array}$ \\
\hline Insulin-like growth factor 1 & IGF1 & $\begin{array}{l}\text { Protein similar to insulin in function and structure } \\
\text { and involded in mediating growth and development. }\end{array}$ \\
\hline Runt-related transciption factor 2 & RUNX2 & $\begin{array}{l}\text { Essential protein in osteoblastic differentiation and } \\
\text { skeletal morphogenensis. }\end{array}$ \\
\hline Secreted phosphoprotein 1 & SSP1 & $\begin{array}{l}\text { Osteopontin. Involved in attachment of osteoclast to } \\
\text { mineralized bone matrix. }\end{array}$ \\
\hline Glyceraldehyde-3-Phosphate Dehydrogenase & GAPDH & $\begin{array}{l}\text { Reference gene. Catalyzes some important steps in } \\
\text { carbohydrate metabolism. }\end{array}$ \\
\hline Actin Beta & ACTB & $\begin{array}{l}\text { Reference gene. Involved in cell structure and } \\
\text { integrity. }\end{array}$ \\
\hline Lactate dehydrogenase $\mathrm{A}$ & LDHA & $\begin{array}{l}\text { Reference gene. Found predominantly in muscle } \\
\text { tissue }\end{array}$ \\
\hline
\end{tabular}

\section{Bone histomorphometry}

Histomorphometry is a quantitative examination of the microscopic organization and structure of a non-decalcified biopsy (as bone and soft tissue). Bone histomorphometry was performed in study II, III and IV to investigate and compare the bone quantity and morphology on PEEK implants, with and without HA coating.

The tibia and femur were dissected and removed from the rabbit and cut into approximately $10-15 \mathrm{~mm}$ thick bone-implant sections. After tissue fixation in $4 \%$ buffered formaldehyde for 24 hours, the samples was increasingly dehydrated in graded ethanol up to $99.9 \%$ (Solveco AB, Sweden). Gradually the ethanol was cleared and the tissue was simultaneously infiltrated with resin without decalcification. Finally, the specimens were embedded in light-curing resin (Technovit 7200 VLC; Heraeus Kulzer, Wehrheim, Germany). The samples were cut and ground using a diamond blade and grinding system (Exakt; Apparatebau, Norderstedt, Germany) into a final thickness of approximately $40 \mu \mathrm{m}$.

In Study III and IV, femur implant with an apical perforation was evaluated. Therefore the cut was aligned along the apical hole in order to evaluate bone area inside the hole.

In study II the samples was stained with Masson-Trichrome-Goldner stain, which has three color staining suitable for distinguishing 
cells and bone from connective tissue ${ }^{220}$. Due to the relatively inertness of PEEK, this staining protocol was selected to reveal non-mineralized and connective tissue in the implant-bone junction.

In study III and IV the samples were stained with stained with Toluidine blue (Pironin G dye, Histolab, Sweden) which stain nucleic acids blue and bone/cartilage in purple. In all studies the samples were imaged using an optical light microscope (Eclipse ME600, Nikon Corporation, Tokyo, Japan) and a digital camera (DS-Ri2, Nikon, Japan). Image analysis and optimization was performed using NiS Elements software (Nikon, Japan). Quantitative histomorphometrical parameters was calculated using Image J.

The following parameters were measured and evaluated:

- Bone-implant contact (BIC, \%): the percentage of bone in contact (visible in the microscope) with the implant surface (Figure 24A). In study IV for the tibia implant evaluation was limited to the top three threads due to the absence of bone in the marrow.

- Bone-area (BA, \%): The percent of bone area within the thread (Figure 24B). In study IV as for BIC in the tibia, the BA was also limited to the top three threads.

- Bone-area inside hole (BAH, \%): The percent of bone area inside the apical perforation (Figure 24C). The perforated implants were exclusively placed in the femur due to the amount and thickness of trabecular bone.

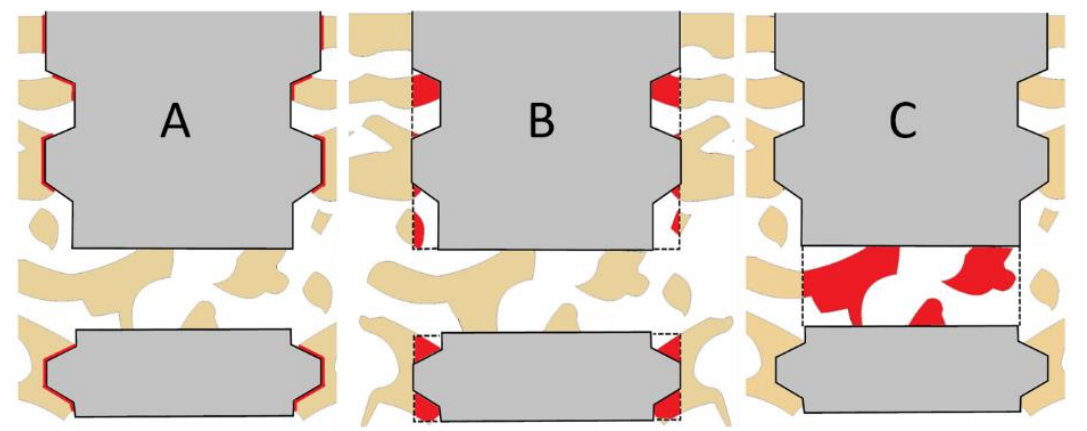

Figure 24. Schematic description of histomorphometric measurement protocol for experimental evaluation of PEEK implants. 


\section{X-ray computed microtomography}

$\mathrm{X}$-ray computed microtomography (micro-CT) is an improvement of conventional X-ray where the physical object is scanned in many cross-sections. The sections can be rendered into a $3 \mathrm{D}$ model with a resolution in the micrometer range. This is a non-invasive technique where the sample is radiated without destroying or modifying the original object.

PEEK is transparent to X-ray which makes it optimal for micro-CT evaluation where the images are not affected by any metal streak artifacts. In study II and study IV, micro-CT was performed to reveal a more comprehensive picture of the bone remodeling around the PEEK implants in addition to conventional histomorphometry.

In study II the micro-CT scanning was performed at the Department of Radiopraxis in Yokosuka, Japan. The samples were scanned as embedded blocks before the cutting procedure. The samples were scanned in atmosphere air and room temperature with a microfocus X-ray CT (MCT-CB100MF Hitachi, Medico, Tokyo, Japan). The implant was placed in a custom jig and aligned in an axial direction vertical to the long axis of the implant. The specimens were scanned with $201 \mu \mathrm{CT}$ slices with a single slice resolution of $51 \mu \mathrm{m}$. X-ray energy level was set to $70 \mathrm{kV}$ with a current of $100 \mu \mathrm{A}$ and scanning time of $120 \mathrm{~s}$. The threshold was calculated by discriminant function analysis, around 2200 voxel value.

The raw data from the X-ray was remodeled into Digital Imaging and Communications in Medicine (DICOM) format, imported and evaluated in a volume analysis program (TRI 3D-BON, Ratoc system engineering, Japan). In the software the implants were positioned to be viewed from the top. To define the Volume of Interest (VOI), the implant midpoint was carefully marked out and the diameter from the center to the thread valley was excluded. The VOI was divided into three adjacent radius volumes with the same length as the implant. The inner region (D1) was selected to have the same thickness as the thread depth $(64$ voxels $=3.26 \mathrm{~mm})$ and the outer two regions (D2, D3) were set to the same thickness as D1 (Figure 25). The bone morphometry as the structural properties of cortical 
and trabecular bone near the implant were evaluated with the following parameters; bone volume to total volume ratio (BV/TV, \%), bone surface per given bone volume (BS/BV, \%), trabecular thickness $(\mu \mathrm{m})$, trabecular number $\left(\mathrm{mm}^{-1}\right)$ and trabecular spacing $(\mu \mathrm{m})$. $\mathrm{BV} / \mathrm{TV}$ indicates the fraction of the selected VOI that is occupied by mineralized bone. BS/BV reflects the bone surface on the trabecular bone in the VOI and can in bone biology provide a value of trabecular bone lining cells for potential osseointegration.

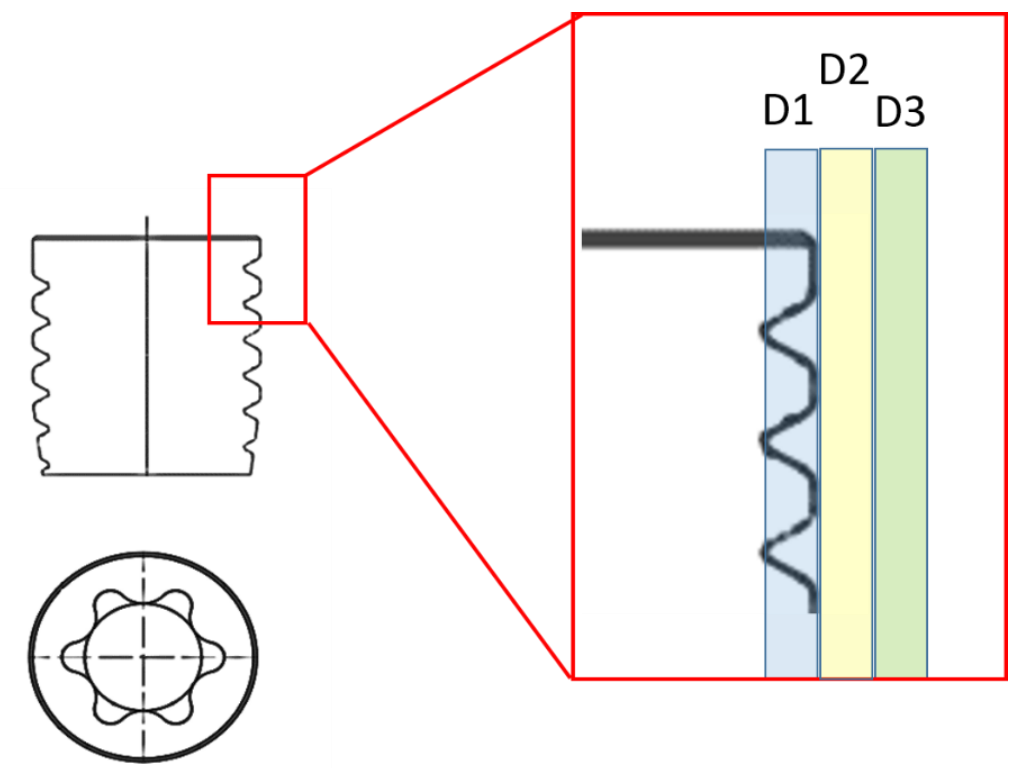

Figure 25. Illustrative division of the VOI's to be examined with micro-CT.

In study IV the micro CT evaluation was performed at the Medical Radiation Physics in Lund. Twelve sections of samples with apical perforation, six test and six control, were imaged with x-ray computed microtomography $(\mu \mathrm{CT})$. The measurements were performed using a ZEISS Xradia Versa 520 X-ray Microscope at the 4D Imaging lab in Lund University, with the source set at $80 \mathrm{kV}$ and $7 \mathrm{~W}$ and with an acquisition time of approximately $2 \mathrm{~h}$ per sample. Each tomography had a voxel size of $5 \times 5 \times 5 \mathrm{~m}^{3}$, with a field of view of roughly $1 \mathrm{~cm}$ in each direction. 
The image analysis was performed with the Amira \& Avizo 3D Software. The high similarity between the absorption coefficients of PEEK and of the light-cured resin, in which the samples were embedded, prevented the use of semi-automatic segmentation techniques. For this reason, the volume of the apical perforations was segmented manually. The volume of the newly formed bone inside the perforations could then be segmented using threshold based methods. Two test samples and one control sample had to be discarded from the subsequent analysis from the $\mu \mathrm{CT}$ scan since they appeared to have been damaged during the sectioning procedure.

The bone volume fraction (BV/TV, \%) inside the apical perforation was calculated from the entrance until a depth of $400 \mu \mathrm{m}$ into the hole. In order to have an indication about the bone growth rate as a function of depth, the concept of cumulative BV/TV was also introduced. It consists in recalculating the BV/TV at each subsequent step of depth ( $5 \mu \mathrm{m}$ step-size), adding progressively to the volume of interest (VOI) the corresponding new slice of volume. At zero depth it coincides with the bone area fraction (BA, \%) at the entrance of the perforation, at every other depth it corresponds to the bone volume fraction in a VOI which goes from the entrance until that particular depth.

\section{Statistics}

Throughout the thesis multiple statistical methods have been applied depending on the number of samples, distribution and type of data. For all statistical methods a difference was considered significant when the P-value was lower than 0.05. All statistical analyses except gene expression data and micro-CT, were performed with SPSS Version 20-22 software (IBM Corporation, Armonk, NY, USA).

In the experimental animal model where RTQ, histomorphometry and micro-CT was evaluated, and test and control could be pairwise compared since both groups were installed in each leg within the same rabbit. Therefore, the groups were considered to be dependent. Due to the limited sample size, the non-normal distribution and the similar dispersion in all groups, the evaluations were submitted to a non-parametric Wilcoxon-signed rank test. An exception appears in 
study III where the result was not possible to be paired within the same rabbit, and therefore the Mann-Whitney test was applied instead of Wilcoxon-signed rank test.

From the topographical evaluation published in study I, the numerical mean values from test and control were considered parametric and analyzed and compared using Student's t-test.

The gene expression analysis was analyzed within the equipment software CFX Manager 3.0 (BioRad, Hercules, CA, USA) where it uses Student's t-test to compare the results from test and control groups.

The results from the micro-CT were averaged between the samples in each of the two groups (HA-PEEK and PEEK); the final results are presented as mean and standard deviation of the mean. A Welch's t-test was used to compare the two groups among them, with a level of significance set at $\mathrm{P}<0.05$. The data analysis was performed in MATLAB environment. 


\section{SUMMARY OF RESULTS}

\section{Surface Characterization}

Investigation of the topographical parameters was performed on the coated and non-coated implants using the optical INF. The scan area was $200 \times 110 \mu \mathrm{m}$ and the resolution allow details on the micrometer level. As the coating was levelling out the microgrooves on the coated samples the $S_{d s}$ was found significantly more $(P=0.002)$ on the uncoated samples. The average deviation in height was similar on both surfaces $\left(\mathrm{S}_{\mathrm{a}}: 0.5-1.0 \mu \mathrm{m}\right)$ classified as minimally rough according to a recognized classification ${ }^{221}$. The topographical parameters are presented in Table 5 .

Table 5. Topographical parameters achieved from INF of PEEK and HA-PEEK presented as mean ( \pm standard deviation) from the top, valley and flank measurements. Neat PEEK possessed significant more density of summits compared to HA-PEEK.

\begin{tabular}{lrrr}
\multicolumn{1}{c}{ Mean \pm SD } & \multicolumn{1}{c}{$S_{\mathrm{a}}(\mu \mathrm{m})$} & \multicolumn{1}{c}{$\mathrm{S}_{\mathrm{dr}}(\%)$} & \multicolumn{1}{c}{$\mathrm{S}_{\mathrm{ds}}\left(\mu \mathrm{m}^{-2}\right)$} \\
PEEK & $0.655(0.231)$ & $53.3(67.7)$ & $185532(38873)$ \\
HA-PEEK & $0.686(0.140)$ & $50.6(23.8)$ & $157794(20781)$ \\
P-value & 0.556 & 0.849 & 0.002
\end{tabular}

To assess and compare the nanostructures of the coated and uncoated surfaces, the samples were surveyed with AFM. The scanning was performed with scan areas of $1 \times 1 \mu \mathrm{m}$ and $10 \times 10 \mu \mathrm{m}$. On both samples nanostructures were detected and on the coated surface the HA crystals were evenly distributed as small summits. The coated surface presented the least height deviation $\left(S_{a}\right)$ and more densely distributed summits $\left(\mathrm{S}_{\mathrm{ds}}\right)$ compared to the control at both scan sizes. 
All results are summarized in Table 6 and rendered 3D images are shown in figure 26.
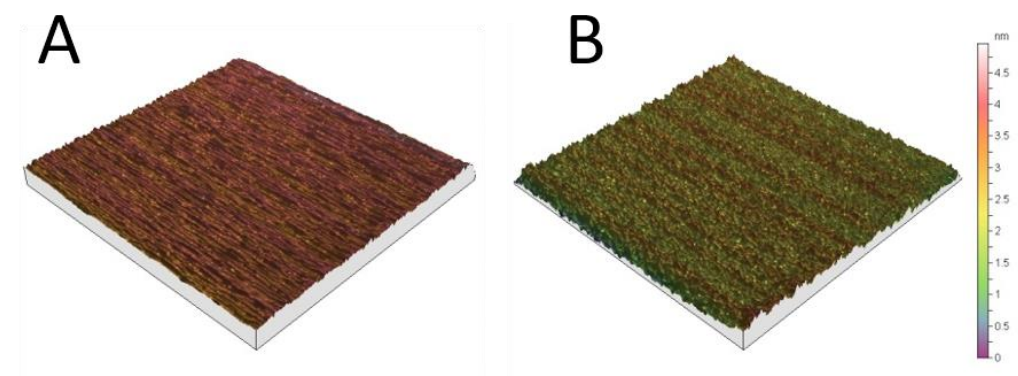

Figure 26. 3D rendering of unmodified PEEK (A) and HA-coated PEEK (B) obtained with AFM with a scan area of $1 \mathrm{x} 1 \mu \mathrm{m}$.

Table 6. Topographical parameters from AFM of PEEK and HAPEEK discs presented as mean ( \pm standard deviation)

\begin{tabular}{lrrrr} 
Scansize & Mean \pm SD & \multicolumn{1}{c}{$\mathrm{S}_{\mathrm{a}}(\mathrm{nm})$} & \multicolumn{1}{c}{$\mathrm{S}_{\text {dr }}(\%)$} & \multicolumn{1}{c}{$\mathrm{S}_{\mathrm{ds}}\left(\mathrm{nm}^{-2}\right)$} \\
$\mathbf{1 \times 1} \boldsymbol{\mu m}$ & PEEK & $1.23(0.58)$ & $4.62(6.59)$ & $3986.3(451.7)$ \\
& HA-PEEK & $0.97(0.46)$ & $2.08(3.67)$ & $4180.8(318.8)$ \\
\multirow{2}{*}{$\mathbf{1 0 \times 1 0} \boldsymbol{\mu m}$} & P-value & 0.160 & 0.484 & 0.799 \\
& PEEK & $12.25(7.57)$ & $0.51(0.60)$ & $11.27(11.00)$ \\
& HA-PEEK & $7.93(6.25)$ & $0.36(0.53)$ & $15.09(13.49)$ \\
& P-value & 0.279 & 0.010 & 0.284 \\
\hline
\end{tabular}

Obtained SEM images of the surfaces showed that the coating procedure was successful in depositing a thin and covering layer of homogenous crystals of HA (Figure 26A-D). The coated implants (B and $\mathrm{D})$ presented a random arrangement of $\mathrm{HA}$ rods covering the surface, whilst the uncoated surfaces (A and C) were relatively smooth at the same magnification. At the micrometer level, both surfaces presented similar roughness. The turning process caused striations and irregularities, which can be seen on the control surface at the lower magnification. The HA coating on the test implants evens out this orientation of surface features.

The contact angle of a surface is affected by the surface preparation, surface roughness and surface energy. Several studies have all 
found a relationship with the aforementioned properties and cell adhesion $^{222}$. The contact angles were measured on the HA-coated and uncoated PEEK discs and were found to be $53^{\circ}$ (SD: 4.4) and $88^{\circ}$ (SD: 2.7), respectively. The HA-PEEK revealed a hydrophilic surface appearance.

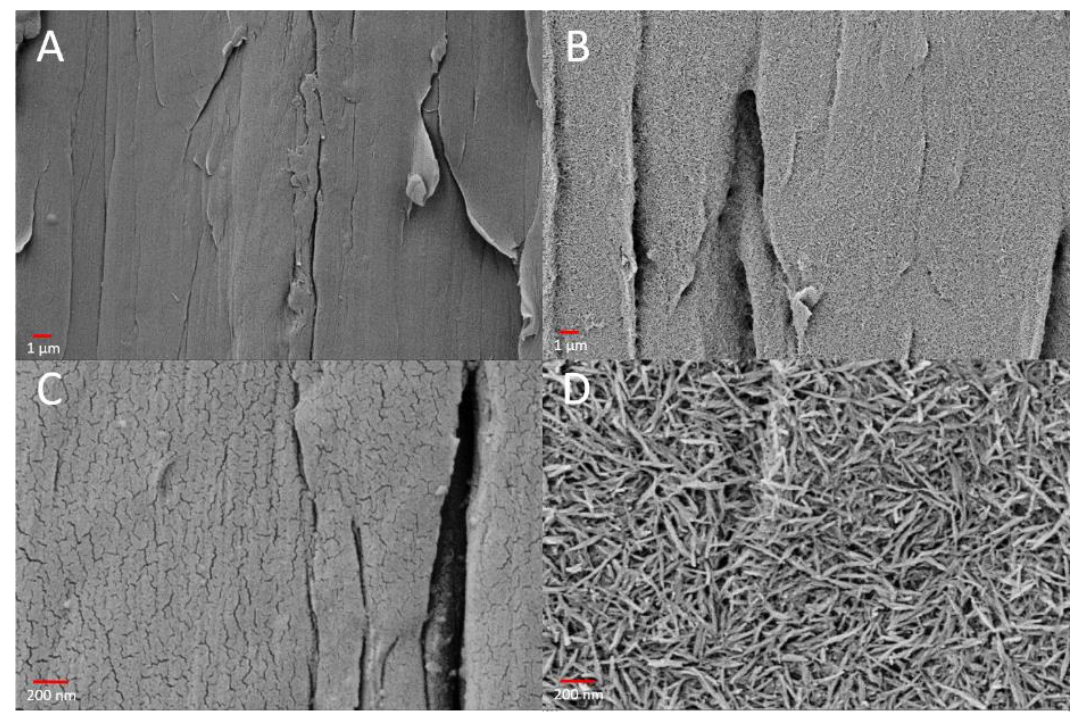

Figure 26. SEM photographs of unmodified (A: 10K, C: $80 \mathrm{~K}$ ) and hydroxyapatite-coated (B: 10K, D: 80K) PEEK implants in two magnifications. Scale bar: $1 \mu \mathrm{m}(\mathrm{A}, \mathrm{B})$ and $200 \mathrm{~nm}(\mathrm{C}, \mathrm{D})$

\section{Chemical and Mechanical Characterization}

The XPS spectra for the coated and uncoated implants are shown in figure $27 \mathrm{~A}$ and $27 \mathrm{~B}$, respectively. Table 7 lists the atomic concentrations calculated from the spectra. Surfaces of the coated samples reveal the presence of carbon, oxygen, and low levels of unexpected contaminations. The coated samples showed the presence of carbon and oxygen, but also calcium and phosphorus from the HA crystals. The coated samples had higher oxygen content, most probably from the HA crystals, which have a theoretical oxygen atomic content of around $62 \%$. The carbon content on the coated samples was lowered from $88.8 \%$ to $55.5 \%$, a logical effect since the carbon-rich PEEK surface was shielded by the HA crystals. The $\mathrm{Ca} / \mathrm{P}$ ratio was 1.41, which differs from the theoretical $\mathrm{Ca} / \mathrm{P}$ ratio for $\mathrm{HA}$ of 1.67 . 
However, due to the semi-quantitative nature of XPS, the difference in $\mathrm{Ca} / \mathrm{P}$ is well within the acceptable tolerance being classified as stoichiometric.
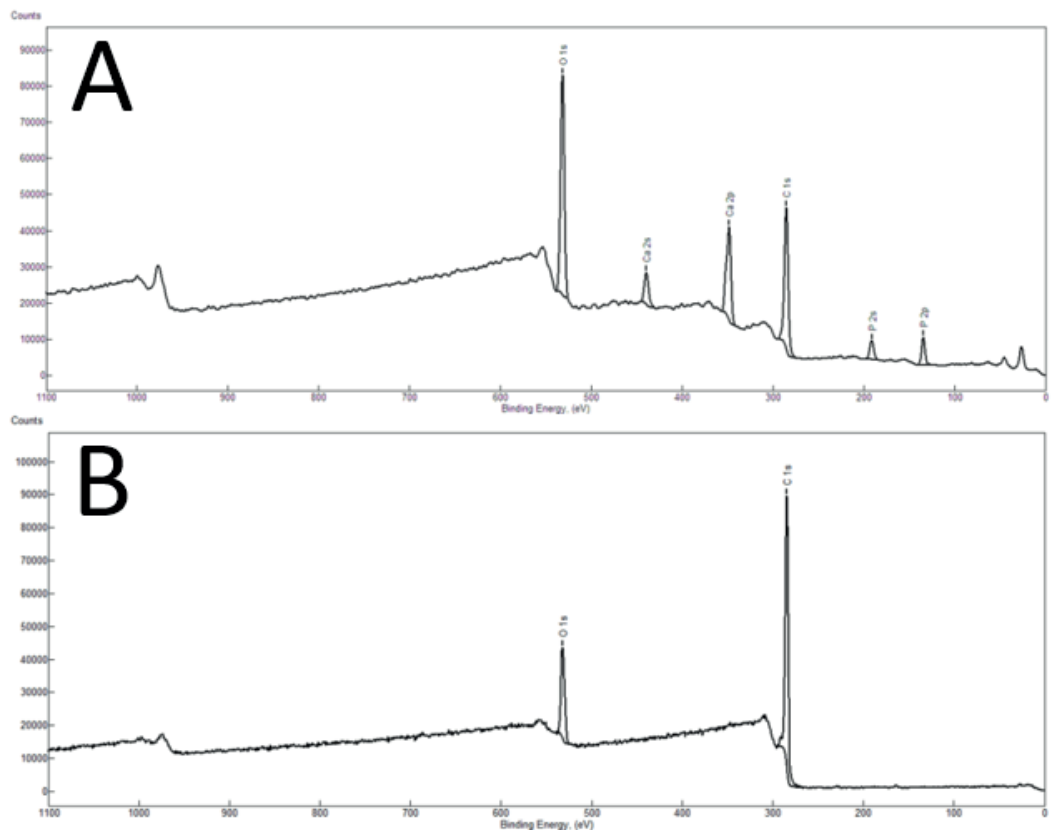

Figure 27. Wide-scan XPS spectrum of hydroxyapatite-coated PEEK (A) and unmodified PEEK (B) showing all elements present before and after the coating procedure.

Table 7. Elemental compositions from the XPS data of HA-PEEK and PEEK.

\begin{tabular}{|c|c|c|}
\hline Atom & PEEK (\%) & HA-PEEK (\%) \\
\hline C & 88.8 & 55.5 \\
\hline $\mathrm{Ca}$ & 0 & 8.8 \\
\hline 0 & 11.2 & 29.5 \\
\hline $\mathbf{P}$ & 0 & 6.2 \\
\hline
\end{tabular}


To reproduce the mechanical stress elicited when HA-coated and control PEEK implants were inserted in Sawbones ${ }^{\circledR}$ material, we monitored the torque during insertion of the implants. Sawbones ${ }^{\circledR}$ consists of a polyurethane foam with a filler, and is available in different porosities and densities, all designed to mimic cortico-cancellous bone proper. Monitoring the torque during the insertion gives an estimate of the mechanical forces on the implant surface. As seen in Figure 28, the insertion torque profiles are virtually the same for coated and uncoated implants. Therefore, even if the coated and uncoated implants have different chemistries and nanostructures, the frictional forces seem most similar for the tested implants. This finding indicates that the friction was governed by the microtopography of the implant surface.

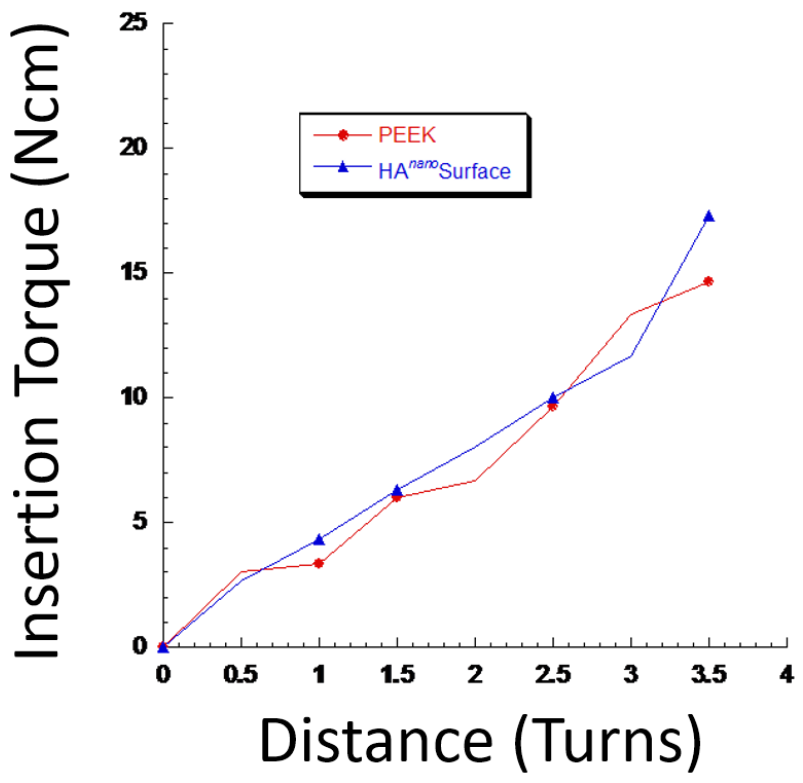

Figure 28. Insertion torque vs. distance for the coated and uncoated implants. The implants were inserted with its top at the level of the surrounding bone surface at 3.5 turns. 


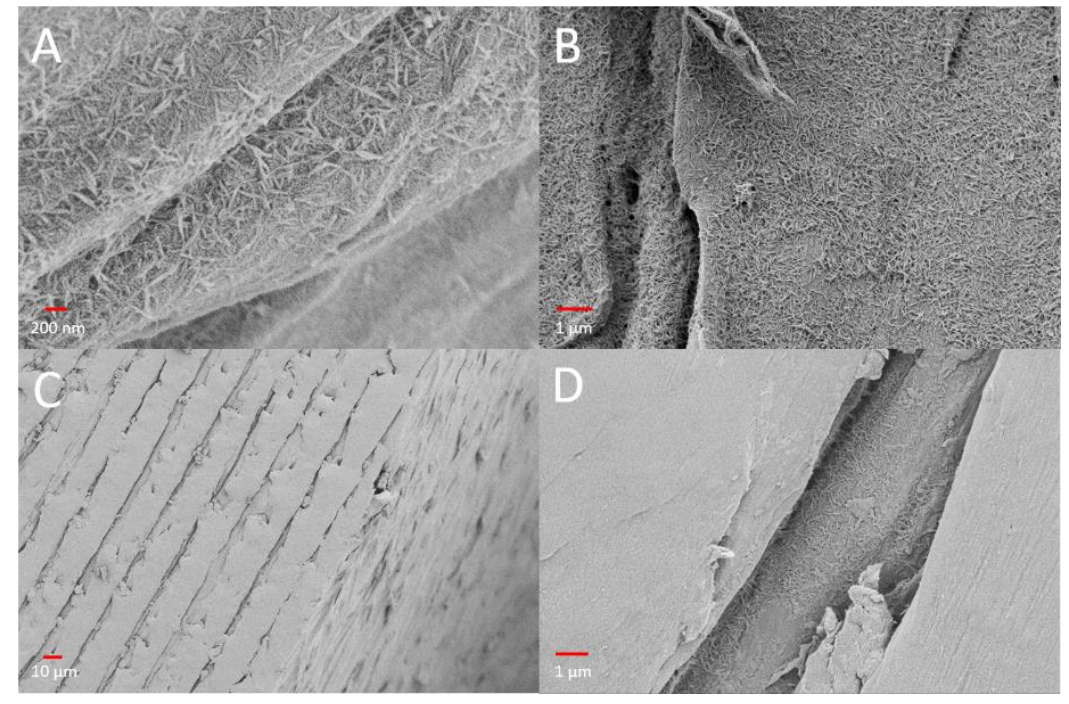

Figure 29. SEM images of a HA-PEEK implant at the thread valleys (A) before insertion and (B) after insertion and removal. The edge of the thread after removal showed absence of mineral. Presented in $1 \mathrm{~K}$ (C) and 20K (D). Scale bar $=10 \mu \mathrm{m}(\mathrm{C}), 1 \mu \mathrm{m}(\mathrm{A}, \mathrm{D})$ and $200 \mathrm{~nm}$ (B).

If the coating is to work properly in a clinical situation, it is important that the majority of the coating can withstand the mechanical forces which are created during the insertion process. Measuring the adhesion strength of the coating to the underlying surface gives valuable information for predicting the durability of the coating. However, standard adhesion measurement protocols for HA coatings such as tensile testing (for example ISO 13779-4 or ASTM F1147-05) are not suitable for coatings in the nanometer regime. These protocols have been suited for plasma sprayed micrometerthick HA-coatings only. A qualitative assessment of the adhesion strength of the HA coating can be done by performing SEM analysis of the implants prior to and after insertion and removal of the implants. As seen in figure 29, the HA coating at the thread valleys was retained after the insertion and removal procedure. The coating was preserved along the thread as well. The thread edges are generally the parts which are subjected to the highest mechanical forces during 
placement and removal of the implant. As appears in Figure 29D, the coating is well-preserved in the small cracks and fissures on the edges, but on the flat areas which were in direct contact with the Sawbones ${ }^{\circledR}$ the PEEK surface showed signs of deformation which, in turn, may lead to low surface coverage of HA crystals (Figure 29C and 29D).

Figures 30A-C show the stress-strain curves obtained from tensile testing. As seen from these figures, the stress-strain performance for the samples are virtually identical. A summary of the tensile testing is presented in Table 8. The elastic modulus was measured to be approximately $4.2 \mathrm{GPa}$, which is in the same range as specified by the PEEK manufacturer. From these data, it can be concluded that the heat treatment that is part of the coating procedure does not alter the mechanical properties of PEEK.
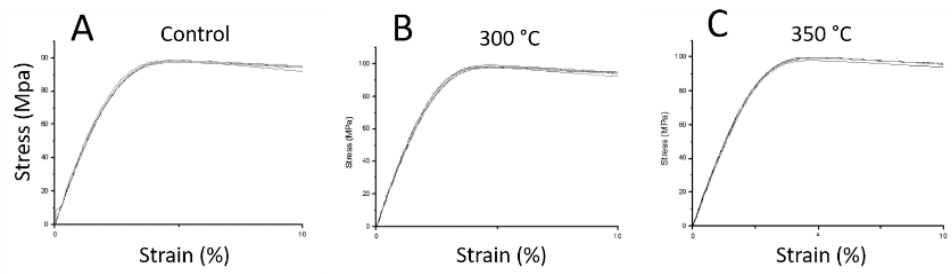

Figure 30. Stress-strain curves for (A) control samples with no heat treatment, (B) $300{ }^{\circ} \mathrm{C}$ and $(\mathrm{C}) 350{ }^{\circ} \mathrm{C}$.

Table 8. Summary of the tensile testing, without heat, at $300{ }^{\circ} \mathrm{C}$ and $350{ }^{\circ} \mathrm{C}$.

\begin{tabular}{|c|c|c|c|c|c|}
\hline Sample & Peak Load (N) & $\begin{array}{l}\text { Peak stress } \\
\text { (MPa) }\end{array}$ & Modulus (MPa) & $\begin{array}{l}\text { Strain at Peak } \\
(\%)\end{array}$ & Break load, (N) \\
\hline Control & 3997 & 98.0 & 4211 & 4.943 & 3418 \\
\hline $300^{\circ} \mathrm{C}$ & 4009 & 98.2 & 4209 & 4.726 & 3384 \\
\hline $350^{\circ} \mathrm{C}$ & 4050 & 99.2 & 4184 & 4.839 & 3390 \\
\hline
\end{tabular}

\section{Ex vivo results}

The implants were retrieved at 3 different healing time points; 3,12 (Study I, II and III) and 20 weeks (Study IV). There was one deceased rabbit of unknown causes from the 3-week group approximately 1 week after the surgery. This rabbit was excluded from all experimental parts of the study. Apart from that incident, all rabbits 
healed uneventfully and did not show any signs of pain or anxiety during the time between implantation and euthanization. The euthanization was performed without complications and at the re-entry there were no manifest signs of infection, bone fractures or stress fractures visible to the eye. At retrieval, all the implant was immobile and no soft tissue infiltration was visible in the bone-implant junction (Figure 31).

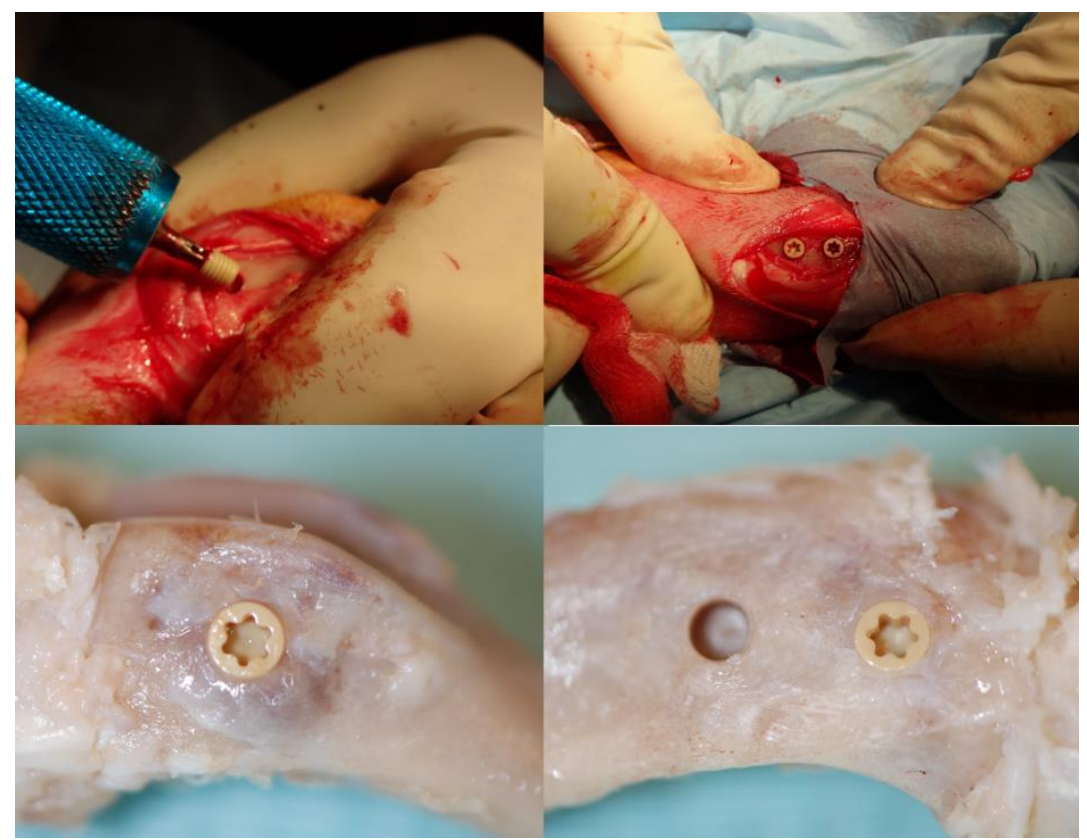

Figure 31. Representative images of the experimental animal surgery. The lower right image shows the removed distally implant after removal torque measure.

\section{Removal torque results}

The RTQ testing was performed subsequently at all healing time points (3,12 and 20 weeks) and the results were published in study I and IV. 
Table 9. Mean and \pm SD removal torque forces $(\mathrm{Ncm})$ after 3 and 12 weeks of healing for PEEK and HA-PEEK.

\begin{tabular}{lrr}
\multicolumn{1}{r}{ Mean \pm SD } & \multicolumn{1}{c}{ 3 weeks } & \multicolumn{1}{c}{12 weeks } \\
PEEK & $7.18(2.96)$ & $5.58(2.07)$ \\
HA-PEEK & $13(5.2)$ & $9.75(4.65)$ \\
P-value & 0.05 & 0.028 \\
\hline
\end{tabular}

In study I, the mean and standard deviation of RTQ measurements for both groups are illustrated in Table 9 and Figure 32. The RTQ values for the uncoated and coated implants after 3 weeks of healing were $7 \mathrm{Ncm}$ and $13 \mathrm{Ncm}$, respectively. After 12 weeks of healing for the uncoated and HA-coated groups, the RTQ values were $6 \mathrm{Ncm}$ and $10 \mathrm{Ncm}$, respectively. The mean values were higher for the HAcoated implants after both healing periods ( 3 weeks: $p=0.01 ; 12$ weeks: $p=0.02$ ) and statistical analysis revealed significant differences between the groups for both evaluation times.

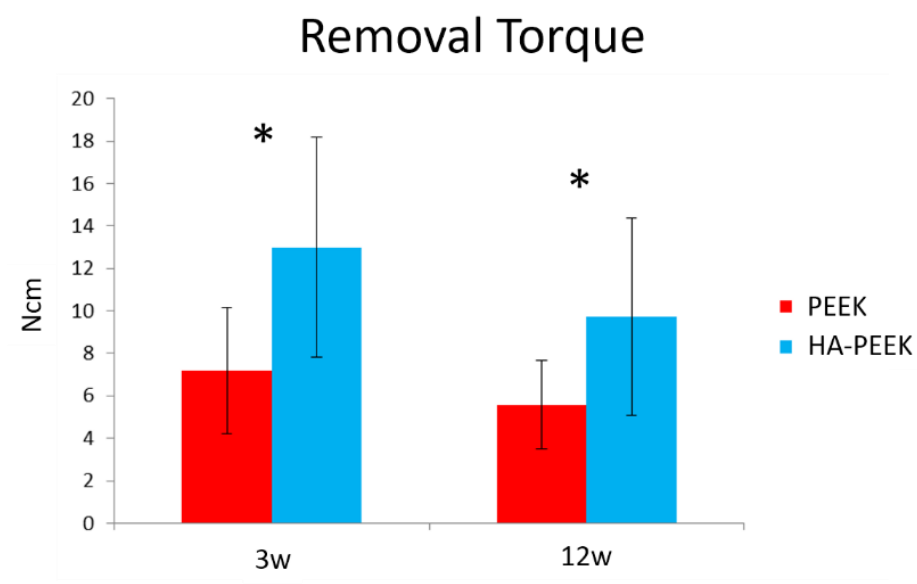

Figure 32. The removal torque values of PEEK (red) and HA-PEEK (blue) after 3 and 12 weeks of healing. ${ }^{*} \mathrm{P}<0.05$. 
The results of study IV is presented in figure 33 where the HA modified PEEK implants from the distal tibia showed a significantly higher removal torque compared to native PEEK implants $(\mathrm{p}=0.03)$. The mean values for HA-coated and uncoated implants were $4 \mathrm{Ncm}$ $(\mathrm{SD}=1.4)$ and $6 \mathrm{Ncm}(\mathrm{SD}=3.3)$, respectively.

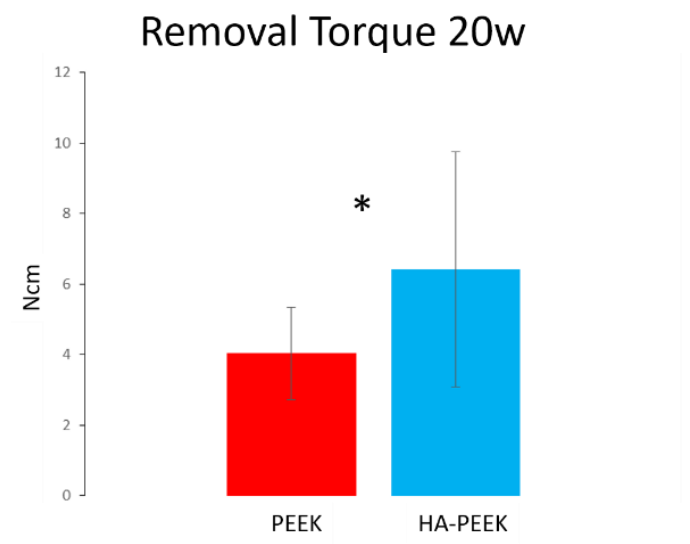

Figure 33. Graph representing the RTQ values for PEEK (red) and HA-PEEK (blue) after 20 weeks of healing. Standard deviation displayed with error bars. ${ }^{*} \mathrm{P}<0.05$.

\section{Gene expression results}

The gene expression of the bone around the removed implants from the 3 weeks healing rabbits were analyzed using a PCR technique. The process showed an up-regulation of several genes that are involved in bone formation (Table 10). BMP2, COL1A1 and IGF1 were all significantly up-regulated on the HA-PEEK implants compared to pure PEEK specimens. A down-regulation was found for RUNX2 for the coated implants without statistically significance. The genes BGLAP and SPP1 were found to be unchanged in the HAPEEK sample when compared to the pure PEEK one. The performance of all the genes can be viewed in figure 34 . 
Table 10. Quantitative gene expression of PEEK and HA-PEEK. The implants were retrieved after 3 weeks of healing. Regulation indicates the fold-difference in expression of PEEK compared to HAPEEK. Significant regulation is high-lighted in red and bold with $\mathrm{p}$ $<0.05$.

\begin{tabular}{|c|c|c|c|c|c|}
\hline Target & Sample & Regulation & $\begin{array}{l}\text { Compared to } \\
\text { Regulation } \\
\text { Threshold }\end{array}$ & P-Value & $\begin{array}{l}\text { Exceeds P- } \\
\text { Value } \\
\text { Threshold }\end{array}$ \\
\hline АСТВ & PEEK & & No change & & No \\
\hline АСТВ & HA-PEEK & & No change & 0,48 & 8 Yes \\
\hline BGLAP & PEEK & 1,00 & No change & & No \\
\hline BGLAP & HA-PEEK & $-1,17$ & No change & 0,11 & 1 Yes \\
\hline BMP2 & PEEK & 1,00 & No change & & No \\
\hline BMP2 & HA-PEEK & 3,19 & Up regulated & 0,03 & $3 \mathrm{No}$ \\
\hline COL1A1 & PEEK & 1,00 & No change & & No \\
\hline COL1A1 & HA-PEEK & 3,22 & Up regulated & 0,03 & 3 No \\
\hline GAPDH & PEEK & & No change & & No \\
\hline GAPDH & HA-PEEK & & No change & 0,26 & 5 Yes \\
\hline IGF1 & PEEK & 1,00 & No change & & No \\
\hline IGF1 & HA-PEEK & 2,49 & Up regulated & 0,00 & No \\
\hline LDHA & PEEK & & No change & & No \\
\hline LDHA & HA-PEEK & & No change & 0,48 & 8 Yes \\
\hline RUNX2 & PEEK & 1,00 & No change & & No \\
\hline RUNX2 & HA-PEEK & $-2,74$ & Down regulated & 0,09 & 9 Yes \\
\hline SPP1 & PEEK & 1,00 & No change & & No \\
\hline SPP1 & HA-PEEK & 1,72 & No change & 0,80 & Yes \\
\hline
\end{tabular}




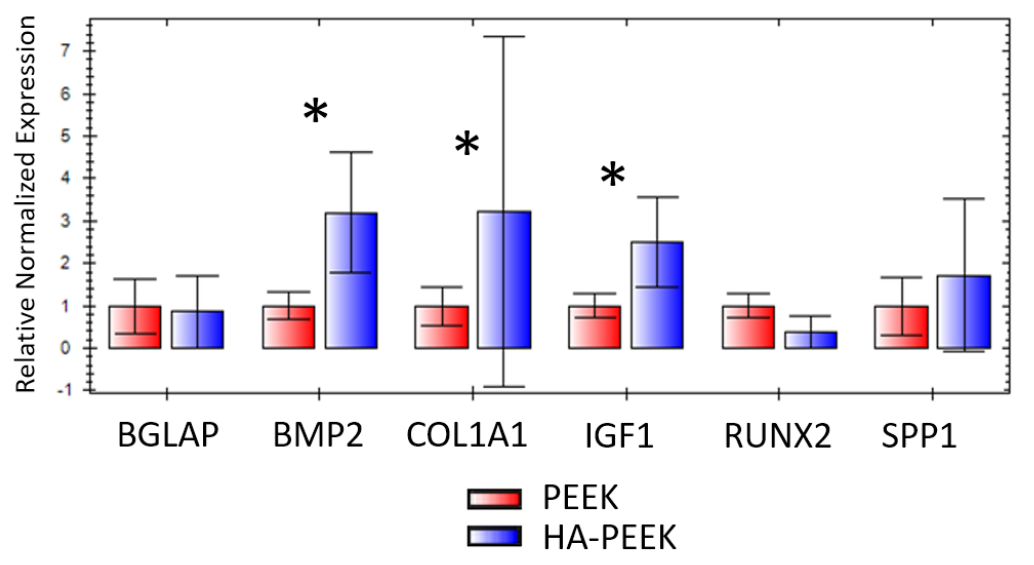

Figure 34. Bar chart showing relative normalized expression of the included genes in tissue from HA-PEEK and PEEK implants after 3 weeks of submerged healing. ${ }^{*} \mathrm{P}<0.05$.

\section{Histological results}

The histologic sections were investigated on the light-microscope and the new bone formation was detected. Two different staining protocols were used in this thesis; Masson-Trichrome-Goldner stain and Toluidine blue stain. After using Masson Trichrome-Goldner stain in the first study a decision was made to continue with Toluidine blue stain since the new/original bone differentiation was easier.

Soft tissue (stained green with Masson-Trichrome-Goldner stain) migration was not seen around any of the examined implants. After 3 weeks the straight bone edge of original bone from the osteotomy was still visible and newly formed bone was emerging against the implant surface. Subsequently, after 12 and 20 weeks of healing mature bone was recognizable inside the treads and inside the perforated hole. Near the implant surface and within the bone undergoing remodeling several Haversian systems were observed (Figure 35). 


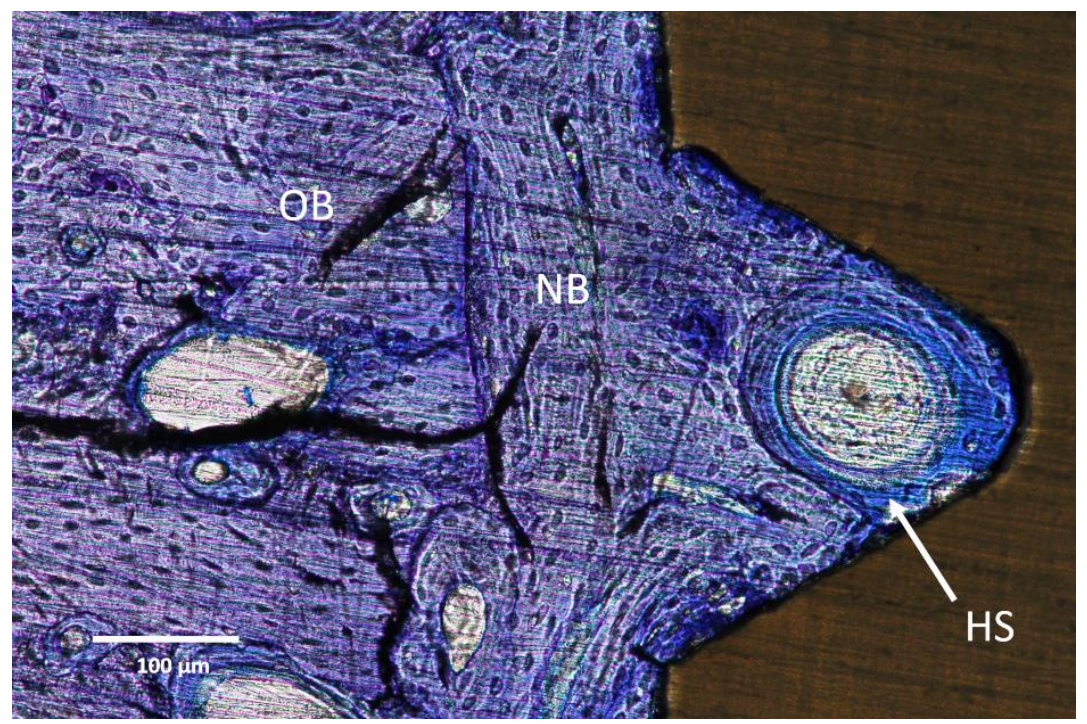

Figure 35. Light microscopy image of section of HA-PEEK after 12 weeks of healing in femur. New bone (NB) and old bone (OB) can be distinguished and inside the thread a Haversian system (HS) was formed. Scale bar $=100 \mu \mathrm{m}$.

In study II, PEEK implants retrieved from 3 and 12 weeks of healing in the tibia were analyzed after the Masson-Trichrome-Goldner stain (Figure 36). The implants were installed so that only one cortical side was involved. The thickness of the cortical plate in a rabbit tibia varies but usually it covers 2-4 threads of the implants used in this thesis. After 3 weeks of healing the average BIC $( \pm \mathrm{SD})$ and BA $\left({ }_{ \pm} \mathrm{SD}\right)$ for the uncoated samples was $2.9 \%(1.9)$ and $17.9 \%(5.8)$, respectively (Table 11). In the coated samples there was an average BIC of $7.9 \%$ (6.9) and BA of $27.5 \%$ (10.6). The average BIC of the coated sample was more than twice the amount of the uncoated one and statistically significant $(\mathrm{p}=0.016)$. In addition, the BA was also significantly higher for coated samples and with less standard deviation $(\mathrm{p}=0.041)$. Results from the 12 week group of implants revealed an average BIC of $4.3 \%$ (3.3) in the uncoated samples, while $6.8 \%(6.5)$ was the average value for the coated ones. The difference was not significant $(\mathrm{p}=0.424)$. The BA demonstrated significant results $(\mathrm{p}=0.041)$ for the coated samples with values of $38.3 \%(9.4)$ 
and $48.7 \%$ (11.1) for uncoated and coated implants, respectively (Figure 36-38, Table 11).

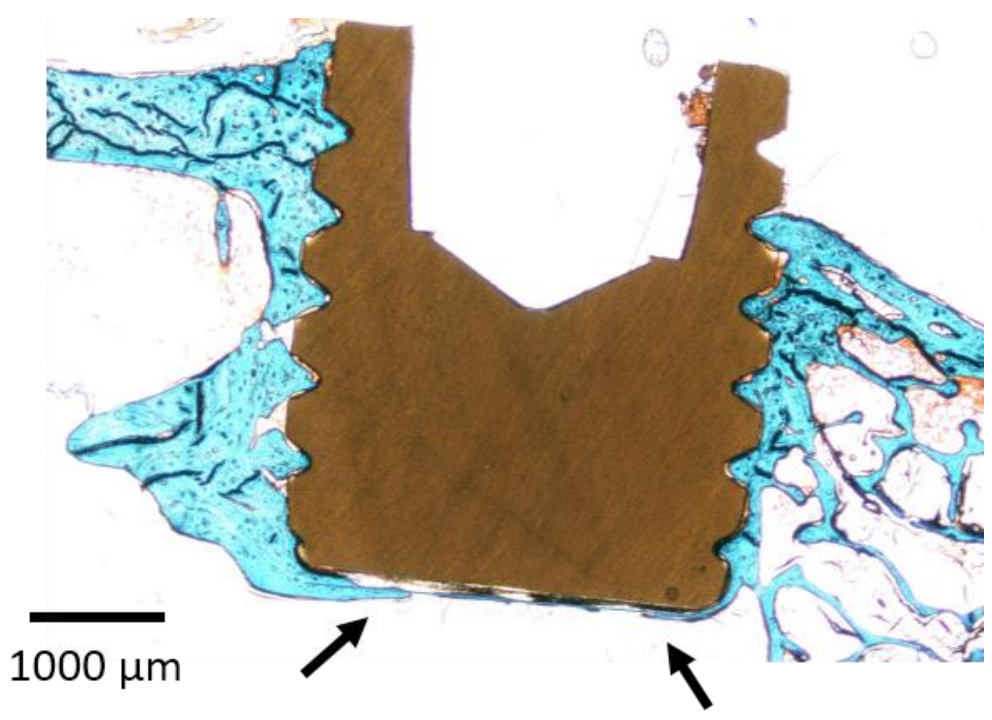

Figure 36. Histologic section of all threads presenting manifest bone ingrowth in all threads, indicative of osteoconductive features of the PEEK material (arrows). This actual section was from an HA-coated implant retrieved 12 weeks after surgery. Staining: Masson-Trichrome-Goldner. Scale bar $=1000 \mu \mathrm{m}$. 


\section{Bone-Implant Contact Tibia}

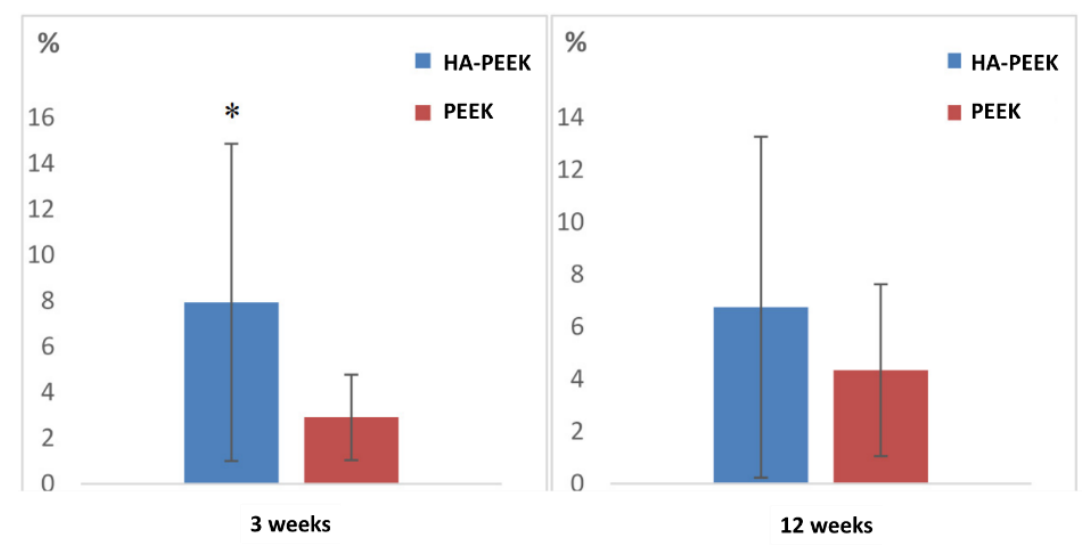

\section{Bone Area Tibia}

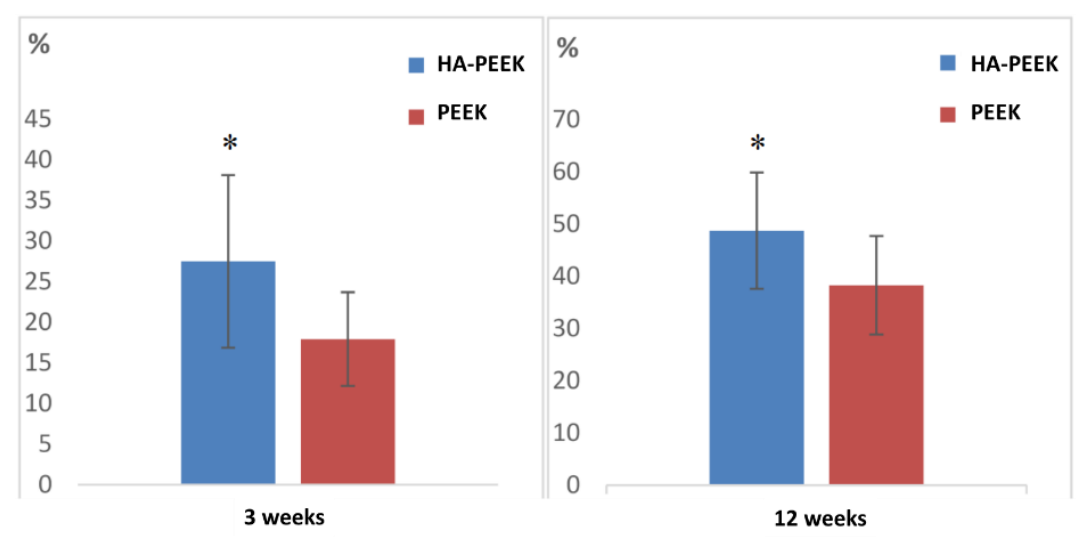

Figure 37. Bar graphs displaying the percentage of (upper) bone area (BA) and (lower) bone-to-implant contact (BIC) for HA-PEEK and PEEK after 3 and 12 weeks of healing in the tibia. Data are presented as mean with standard deviation. (*, $\mathrm{p} \leq 0.05)$. 
Table 11: Histomorphometrical parameters of HA-PEEK and PEEK implanted in tibia after 3 and 12 weeks of healing. Published in study II. $\mathrm{P}<0.05$ marked in bold.

\begin{tabular}{|l|l|c|c|}
\hline $\mathbf{n = 2 4}$ & & Bone-implant contact (BIC) & Bone area (BA) (in thread) \\
\hline \multirow{3}{*}{ 3 weeks } & Test & $\mathbf{7 , 9 4}(6,94)$ & $27,49(10,63)$ \\
& Control & $2,91(1,87)$ & $17,91(5,78)$ \\
& p-value & $\mathbf{0 , 0 1 6}$ & $\mathbf{0 , 0 4 1}$ \\
\multirow{3}{*}{$\mathbf{1 2}$ weeks } & Test & $6,75(6,52)$ & $48,72(11,12)$ \\
& Control & $4,34(3,28)$ & $38,27(9,43)$ \\
& p-value & 0,424 & $\mathbf{0 , 0 4 1}$ \\
\hline
\end{tabular}

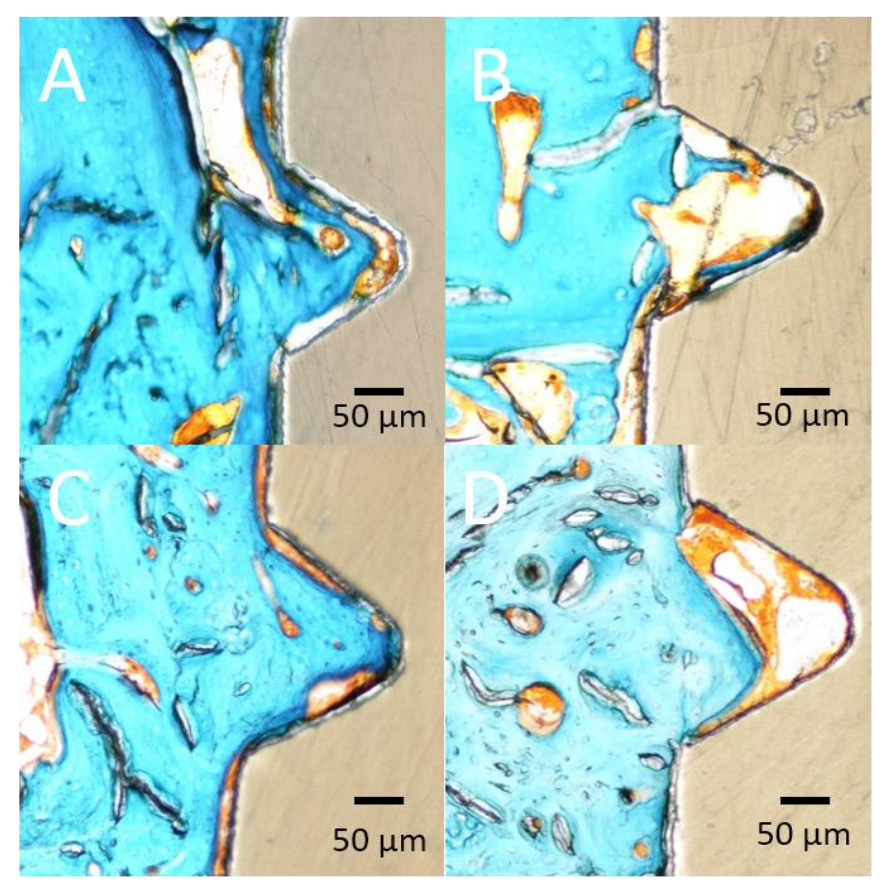

Figure 38. Histological pictures of Masson-Trichrome-Goldner stained sections of the implants after 3 and 12 weeks of healing. Fibrous tissue (orange, arrow) can be viewed at the gap between the bone and implant, more commonly at the 3 week samples. (A): 3 weeks HA-PEEK, (B): 3 weeks PEEK, (C): 12 weeks HA-PEEK, (D): 12 weeks PEEK. Scale bar $=50 \mu \mathrm{m}$. 
In study III, the histomorphometrical analysis was carried out on implants placed in the femur bone. The healing time was the same as in study II, 3 and 12 weeks but the implants were designed with an apical perforation. Three weeks after insertion the HA-coated sample (Figure 39A) showed more osteocytes and lamellar bone compared to the pure PEEK (Figure 39B). After 12 weeks of healing the HA-coated implant (Figure 39C) showed signs of vital and rapid bone formation with a Haversian systems inside the thread surrounded by large amounts of osteocytes. The uncoated PEEK implant after 12 weeks demonstrated large amounts of osteocytes but with a lower extent of bone-implant contact (Figure 39D).

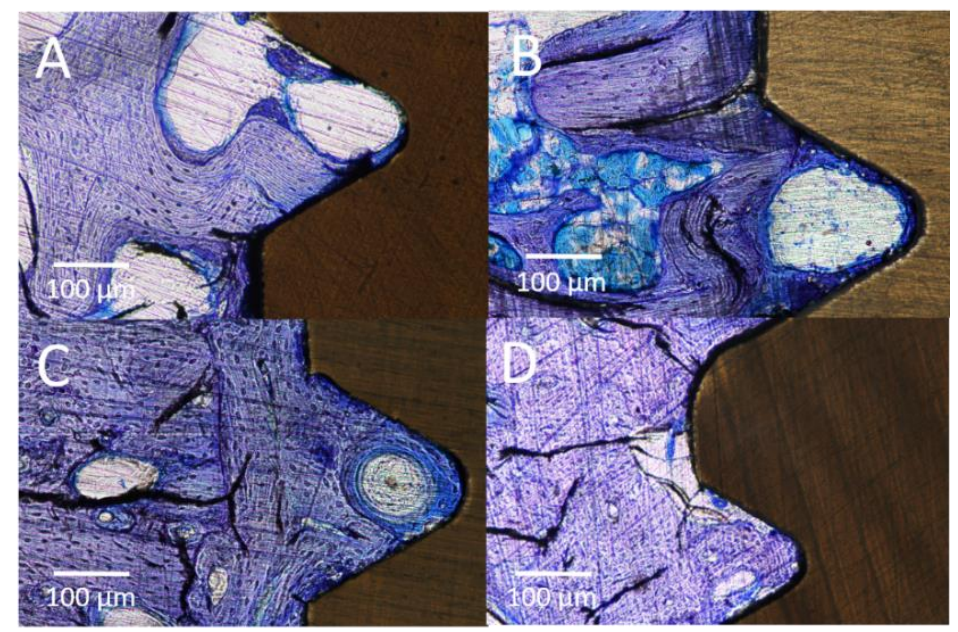

Figure 39. Histological sections after 3 and 12 weeks of healing in the femur bone. (A): 3w HA-PEEK, (B): 3w HA-PEEK, (C): 12w HA-PEEK, (D): 12w PEEK. Scale bar $=100 \mu \mathrm{m}$.

The observed histomorphometrical values from the threads in study III showed slightly different outcomes compared to the results from study II. After 3 weeks of healing the average BIC and BA for the uncoated samples was $11.1 \%$ (3.5) and $25 \%$ (7.6), respectively (Table 12). In the coated samples there was an average BIC of $14.1 \%$ (3.5) and BA of $27.7 \%$ (8.3). The difference was statistically significant for the average BIC values $(\mathrm{p}=0.034)$ but not for the average BA values $(\mathrm{p}=0.478)$. 
Table 12. Histomorphometric results from femoral implants after 3 and 12 weeks of healing. $\mathrm{P}<0.05$ marked in bold.

\begin{tabular}{|c|c|c|c|c|}
\hline 3 week & & BIC (\%, SD) & $\begin{array}{l}\text { BA-thread (\%, } \\
\text { SD) }\end{array}$ & BA-hole $(\%, S D)$ \\
\hline & PEEK & $11.1(3.5)$ & $25.0(7.6)$ & $4.5(2.9)$ \\
\hline & HA-PEEK & $14.1(3.5)$ & $27.7(8.3)$ & $17.2(7.8)$ \\
\hline & p-value & 0.034 & 0.478 & 0.000 \\
\hline \multicolumn{5}{|l|}{12 week } \\
\hline & PEEK & 11.4 (3.8) & $44.5(10.8)$ & $10.8(5.5)$ \\
\hline & HA-PEEK & $16.7(6.7)$ & $49.7(13.5)$ & $21.3(6.9)$ \\
\hline & $p$-value & 0.038 & 0.211 & 0.001 \\
\hline
\end{tabular}

Results from the 12 week retrieved implants demonstrated an average BIC of $11.4 \%$ (3.8) in the uncoated samples, while $16.7 \%$ (6.7) was the average value for the coated ones. The difference was statistically confirmed ( $\mathrm{p}=0.038)$. The BA for the coated sample was not significantly higher $(\mathrm{p}=0.211)$ than the uncoated ones with average values of $44.5 \%$ (10.8) and $49.7 \%$ (13.5) for uncoated and coated, respectively (Figure 40 and 41 ).

\section{Bone-Implant Contact}

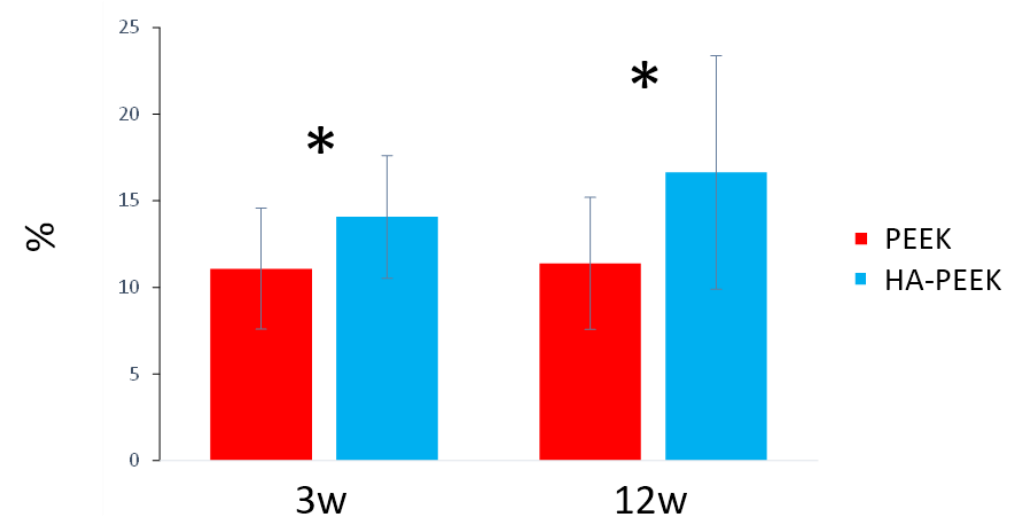

Figure 40. Bar chart of bone-implant contact in femur after 3 and 12 weeks of healing. ${ }^{*} \mathrm{P}<0.05$. 


\section{Bone Area Thread}

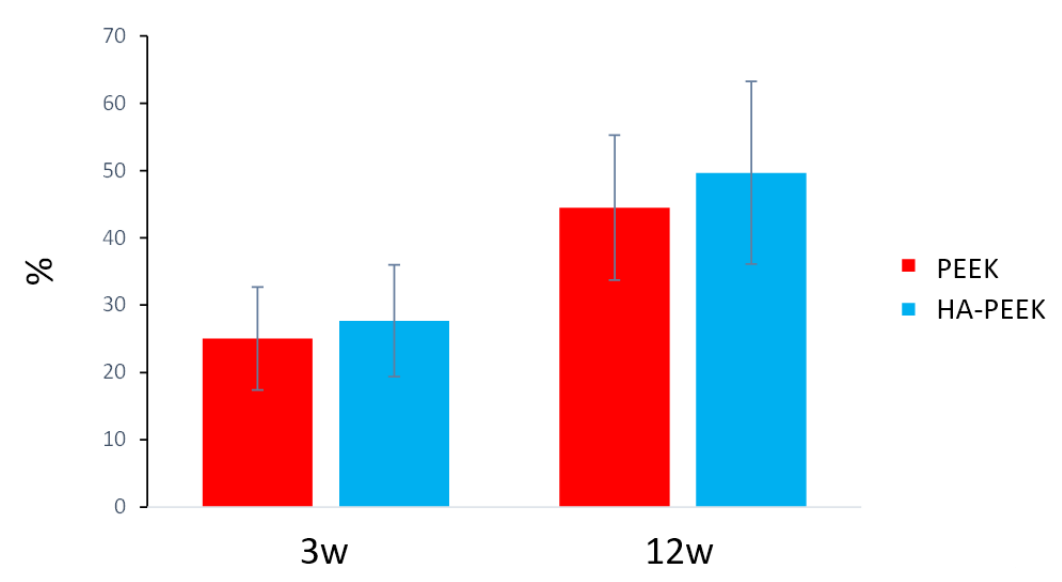

Figure 41. Bar chart of bone area inside the thread in femur after 3 and 12 weeks of healing.

Supplementary in study III, the bone formation was evaluated inside the perforated hole. Histological sections in two magnifications investigated the apical perforation of HA-coated (Figure 42A-B) and uncoated (Figure 42C-D) PEEK implant, three weeks after insertion. The HA-coated implant in lower magnification (A) showed bone growth through the entire hole. In the enlarged image (B) the line between new bone and old bone was tracked and inside the hole the bone approached the implant surface with regular intervals through the hole. The uncoated PEEK implant demonstrated significantly less bone area at the entrance and inside the perforated hole compared to HA-coated PEEK (C). With higher magnification the bone was found to be located in the center of the perforation without having contact to the implant surface (D). The histomorphometrical results from the apical perforation revealed a very significant difference in favor of the coated samples (Figure 43). After 3 weeks of healing the BA was $4.5 \%(2.9)$ and 17.2 (7.8) for coated and uncoated implants, respectively. Implants harvested after 12 weeks showed an average BA of $21.33 \%$ (6.9) and $10.8 \%$ (5.53) for coated and uncoated samples, respectively (Table 12). 


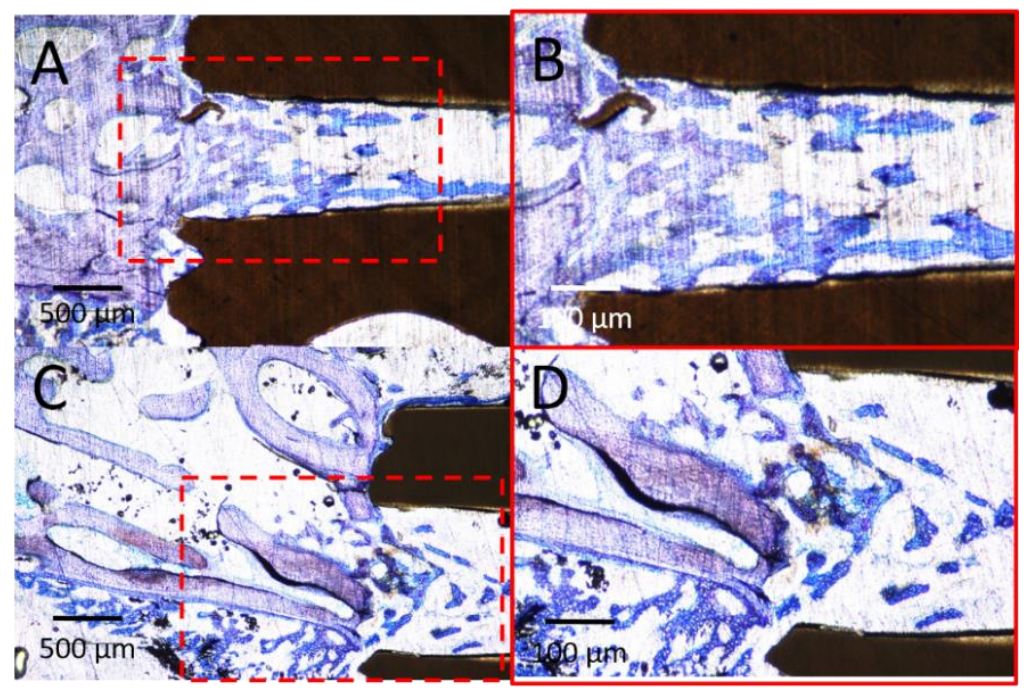

Figure 42. Histological sections in two magnifications showing the apical perforation of HA-coated (A-B) and uncoated (C-D) PEEK implant, 3 weeks after insertion. The HA-coated implant in lower magnification (A) show bone growth through the entire hole. In the enlarged image (B) the line between new bone and old bone can be tracked and inside the hole the bone approaches the implant surface with regular intervals through the hole. The uncoated PEEK implant demonstrates significant less bone area at the entrance and inside the perforated hole compared to HA-coated PEEK (C). With the higher magnification the bone structure was found to be located in the center of the perforation without having contact to the implant surface (D).

In study IV, the bone formation was evaluated after 20 weeks of healing with a more extensive bone maturation. In the qualitative evaluation woven bone and lamellar bone were found in contact with the implant surface inside the threads and at the external surfaces. The quantitative evaluation after 20 weeks of healing in rabbit femur revealed higher BA and BIC values for test implants but without statistical significance (BA: $\mathrm{P}=0.213$, BIC: $\mathrm{P}=0.441$ ). In the tibia there was no significant difference of $\mathrm{BA}$ and $\mathrm{BIC}$ values between the groups when measuring the whole part of the implant. Bone area and BIC in the top three threads of tibia after 20 weeks 
were higher for the test group with values of $58,2 \%$ (BA) and 21.8 $\%$ (BIC) compared to values of $53.1 \%(\mathrm{BA})$ and $17.9 \%$ for the control (Table 13).

Table 13. Histomorphometric results from tibial and femoral implants after 20 weeks of healing.

\begin{tabular}{|r|rrrrr|}
\hline \multirow{2}{*}{ Location } & \multicolumn{2}{c}{ Group } & \multicolumn{2}{c}{ TIC } & \multicolumn{2}{c|}{ Top3-BIC } & \multicolumn{2}{c|}{ Top3-BA } \\
\hline Tibia & Test & 0,13 & 0,22 & 0,38 & 0,58 \\
\hline & Control & 0,15 & 0,18 & 0,41 & 0,53 \\
\hline & P-value & 0,09 & 0,11 & 0,17 & 0,07 \\
\hline \multirow{2}{*}{ Femur } & Test & 0,19 & & 0,57 & \\
\hline & Control & 0,17 & & 0,51 & \\
\hline & P-value & 0,44 & & 0,21 & \\
\hline
\end{tabular}

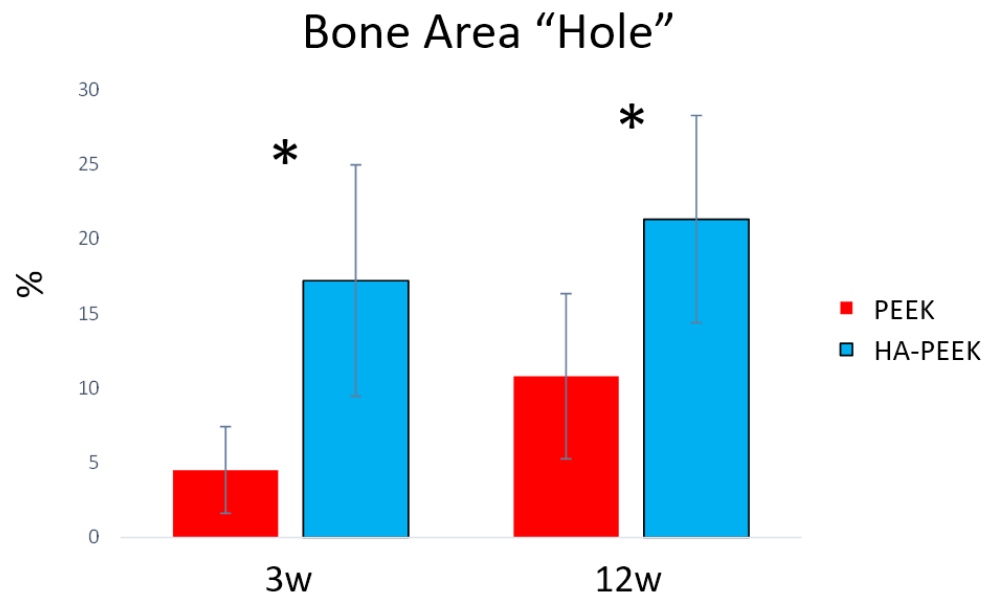

Figure 43. Bar chart of bone area inside the perforated hole in femur after 3 and 12 weeks of healing. ${ }^{*} \mathrm{P}<0.05$.

\section{Micro-CT results}

The three-dimensional evaluation of the femur implants permits a more comprehensive evaluation of the bone volume inside the apical perforation. The reconstructed images further provided imaging with high resolution and contrasts. Since PEEK is transparent to $\mathrm{X}$ rays, the reconstructed images were free from streak artifacts. 
The results acquired from 3 and 12 weeks healing showed for the full volume of interest (VOI, D1 + D2 + D3) no significant differences between the coated and uncoated implants (Figure 44). The inner VOI (D1 closest to the implant thread) revealed, after 12 weeks only, a significant difference $(\mathrm{p}=0.044)$ between the coated and uncoated implant when comparing bone surface in terms of volume (HA: $23.5 \% \pm 1.5 \%$, PEEK; $24.7 \% \pm 4.3 \%$ ) and trabecular thickness (HA: $85.5 \pm 5.4 \mu \mathrm{m}$, PEEK: $82.8 \pm 12.1 \mu \mathrm{m}$ ). This difference aside, there were no other significant differences between the groups or healing periods (Table 14).

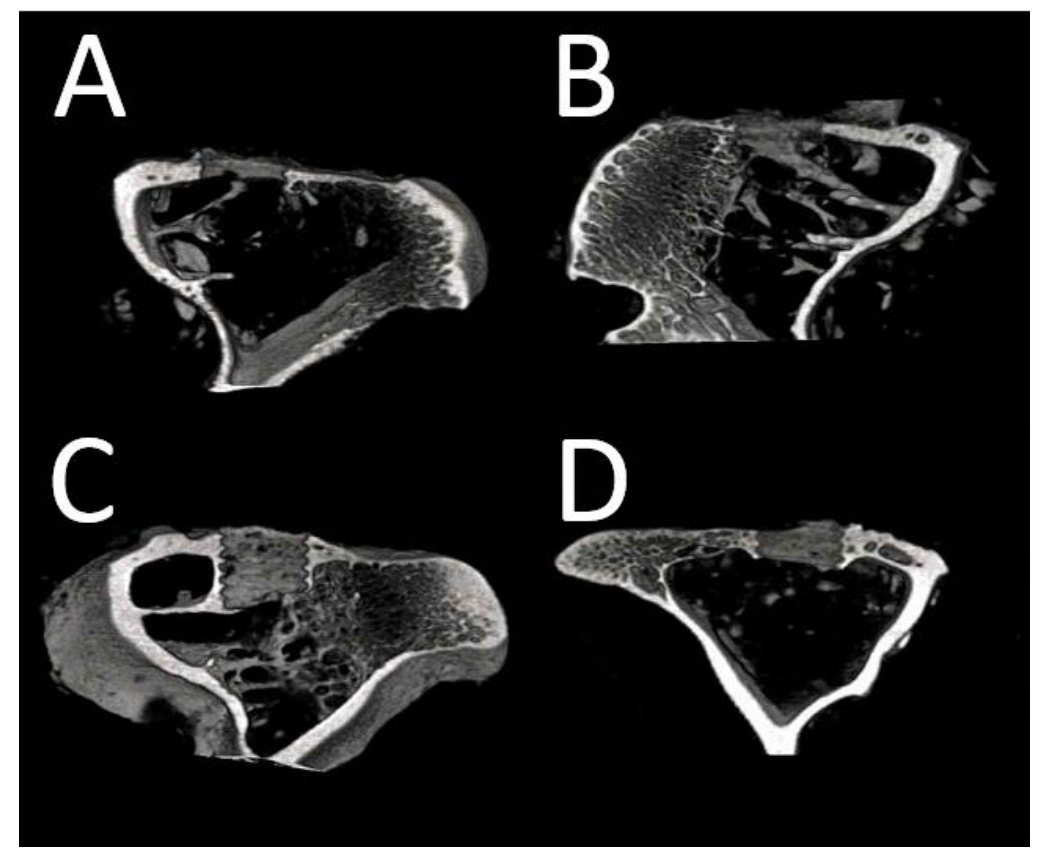

Figure 44. Micro-CT results. Three dimensional reconstruction illustrating the appearance of the implant and bone, 3 and 12 weeks after surgery. A: 3 weeks control, B: 3 weeks test (HA), C: 12 weeks control, D: 12 weeks test (HA). The PEEK implant appears radiolucent to X-ray as transparency in the bone. 
Table 14. Numerical outcomes from micro-CT analyses of PEEK and HA-PEEK implants at 3 and 12 weeks of healing.

\begin{tabular}{|c|c|c|c|c|c|c|}
\hline \multirow{2}{*}{\multicolumn{2}{|c|}{ Group D1+D2+D3 $(n=24)$}} & \multicolumn{5}{|c|}{ Average (SD) } \\
\hline & & BV/TV (\%) & BS/BV (\%) & Tb.Th $(\mu \mathrm{m})$ & Tb.N $\left(\mathrm{mm}^{-1}\right)$ & Tb.Sp ( $\mu m)$ \\
\hline \multirow{3}{*}{3 weeks } & HA-PEEK & $28,0(11,3)$ & $21,3(4,0)$ & $96,8(15,8)$ & $2,8(1,0)$ & $305,2(170,2)$ \\
\hline & PEEK & $26,0(10,9)$ & $21,6(4,9)$ & $96,8(18,9)$ & $2,6(0,9)$ & $340,5(190,1)$ \\
\hline & $\mathrm{p}$-value & 0.982 & 0.123 & 0.125 & 0.659 & 0.510 \\
\hline \multirow{3}{*}{12 weeks } & HA-PEEK & $42,7(0,2)$ & $19,6(3,1)$ & $104,2(15,6)$ & $4,0(1,3)$ & $171,3(97,0)$ \\
\hline & PEEK & $41,2(0,2)$ & $19,7(4,4)$ & $105,5(18,8)$ & $3,8(1,1)$ & $184,8(118,6)$ \\
\hline & $p$-value & 0.451 & 0.277 & 0.554 & 0.067 & 0.928 \\
\hline
\end{tabular}

The results from the 20 weeks mean value of the BV/TV in a $400 \mu \mathrm{m}-$ deep VOI inside the apical perforation was $27 \%( \pm 4 \%)$ for the HAPEEK samples and $21 \%( \pm 8 \%)$ for the PEEK ones (Figure 45$)$. The comparison between the two groups showed a p-value of $\mathrm{P}=0.56$, indicating that the low number of samples did achieve statistically meaningful conclusions or that there were no differences. The mean cumulative BV/TV, presented in figure 46 , does not show significant differences in the behavior of the two groups. The lower variance at higher depths seems to indicate that the reduction rate of the BV/TV is faster for samples with high starting values of BV/TV.

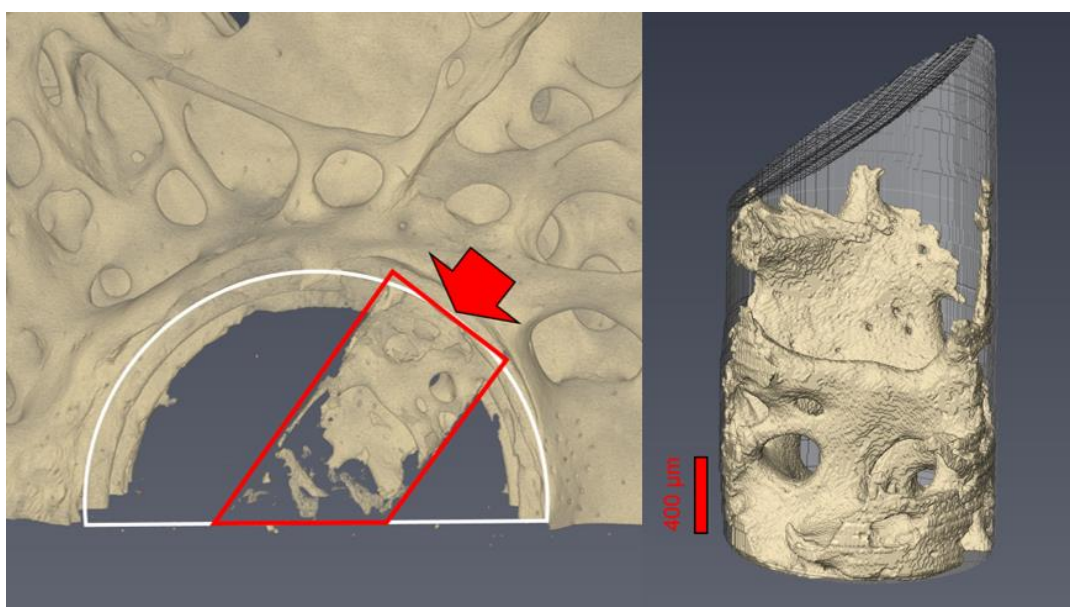

Figure 45. 3D rendering of a $\mu \mathrm{CT}$ scan, viewpoint along the rotation axis of the implant. The section of the PEEK implant cannot be distinguished from the background, and appears as a hollow semi-cylindrical area (white outline) in the bone structure; the red outline 
delimits the apical perforation, of which the red arrow marks the entrance. Right - Segmentation of the apical perforation (in transparent light grey) and of the bone inside it. The red bar shows the scale $(400 \mu \mathrm{m})$.

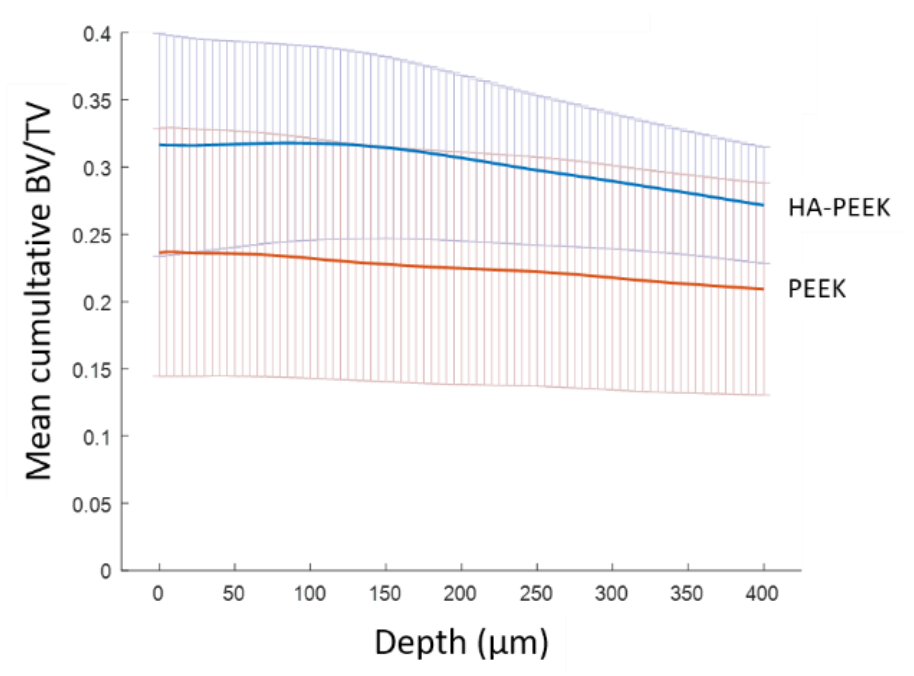

Figure 46. The mean cumulative BV/TV as a function of the depth inside the apical perforation, for the test and the control samples. The standard deviation of the mean is displayed as error bars. 


\section{DISCUSSION}

The subsequent discussion section will be based on the results and conclusions made out of the four experimental studies. The data was gathered from three sessions of rabbit surgery where HA-PEEK and pure PEEK implants were inserted into rabbit bone. Implants were retrieved at different healing times to distinguish the different stages of healing and osseointegration. Several ex vivo and in vitro analytical methods have been employed to evaluate the effect of the surface modification.

Dental and orthopedic implants in situ are used to replace or support hard or soft tissue structures and transfer the applied load to the surrounding bone. Maintaining the bone volume and bone strength around the implant is a prerequisite for a successful treatment. According to Albrektsson et al. ${ }^{223}$ there are six factors that determine the host and bone response to an implant:

- Biocompatibility

- Implant surface

- Implant design

- State of the host bed

- Surgical technique

- Loading conditions

This thesis will focus on the biocompatibility and the implant surface since the other factors have been controlled in the two implant groups.

\section{Structural and chemical properties of the HA-coating}

Hydroxyapatite is a common and successful coating material on implants due to its biocompatibility. In this thesis a synthetic nanosized crystalline calcium phosphate coating was evaluated. The most common coating techniques such as sputter coating ${ }^{224,225}$, sol-gel coating $^{226,227}$ and dip-coating ${ }^{228,229}$ normally create a coating as thick 
as $100 \mu \mathrm{m}$ and usually alter the micro-roughness ${ }^{230,231}$. Some of these techniques have demonstrated poor adhesion of the coating which has resulted in loosening of coating particles into the surrounding bone and tissue. These unattached particles have in several clinical studies been shown to cause progressive bone resorption adjacent to the implants ${ }^{232,233}$. A study by Hedia ${ }^{234}$ concluded that the coating thickness affects the stability of the coating to the surface. The form and shape of a coating affects the peak stress to the surface under parallel shear stress, not the absolute height of the coating ${ }^{235}$. An example to explain the theory: If a surface has $S_{\mathrm{ds}}=1$ and another surface has $S_{\mathrm{ds}}=2$, on a definite area the " $\mathrm{S}_{\mathrm{ds}}=1$ "-surface will have fewer summits per unit area compared to the " $S_{d s}=2$ "-surface. Regardless of the roughness value $\left(\mathrm{S}_{\mathrm{a}}\right)$ of the surfaces, it will always be more summits per square unit on the " $\mathrm{S}_{\mathrm{ds}}=2$ "-surface. Thus, different coating techniques may produce the same coating thickness but with a different adhesion to the surface. Therefore, the literature suggests that a thin and moderately smooth coating surface is optimal for providing a favorable bone response.

It is important to thoroughly characterize the surface properties of the specimens to be evaluated and compared. The INF and AFM were used in this study to quantify and illustrate the micro- and nanotopography of the samples. The HA coating showed no considerable effect on the surface microtopography $\left(S_{a}, S_{d r}\right)$ measured with the INF. However, the density of summits was significantly higher for the uncoated samples. The applied coating was covering the entire surface and the micro-cracks and grooves were plugged and therefore fewer summits are visible on the coated samples. The nanotopography was highlighted using AFM and in contrast to the INF the density of summits was higher on HA-PEEK. The resolution of the AFM is far higher than INF and can detect the summits of the coating minerals. The surface data from INF and AFM are used to characterize the surface and compare the applied coating with the neat sample. However, it is unknown whether the small differences in surface roughness, especially $S_{\mathrm{ds}}$ and $S_{\mathrm{dr}}$, will affect the bone response and cellular behavior.

The spin-coating technique used in this study has been evaluated by other authors in vivo on titanium implants ${ }^{236-239}$. The mineral size 
and coating technique were shown to be capable of producing a surface with nanotopography ${ }^{236}$. After 4 weeks of healing there was an increased implant retention of the implant surface with nano topography. The same research group evaluated electro-polished titanium implants, one modified with nanosized HA and the other one left intact as control ${ }^{237}$. The results showed increased early bone formation on the implant with nano-topography with significantly increased BIC for nano-HA implants. The study by Jimbo et al. ${ }^{238}$ compared three different calcium phosphate coatings with nanostructures on relatively smooth implants with respect to RTQ and histomorphometry. Uncoated implants were used as control. The implants without any noticeable HA minerals and the implants with long and straight minerals possessed significantly higher retention in bone compared to controls. The results were a surprise since all groups had similar surface roughness. The same authors compared sand-blasted and acid-etched titanium implants implanted in rabbit bone. Half the numbers of implants remained without HAcoating $^{239}$. In terms of BIC there were no significant difference between the groups.

Some of the aforementioned studies evaluated the bone formation around implants using conventional histomorphometry. Some results indicated that different chemical compositions of the implant surface affected the early bone response. Meirelles et al. ${ }^{237}$ revealed a threefold increase on BIC around HA-implants compared to untreated ones $(\mathrm{P}=0.038)$. Nonetheless, the $\mathrm{BA}$ values showed similar results between the groups. The authors described a possible explanation where the nano HA surface at the early healing times only altered the bone response at the implant surface, not the bone density. Several other studies have found a similar trend where BIC differs between different implant surfaces, but the BA remains relatively constant $^{240-242}$.

Study II, III and IV evaluated the bone formation after different healing times using quantitative histomorphometry. The results from study II showed significantly higher BIC on HA-PEEK compared to uncoated implants at 3 weeks of healing. This resembles the aforementioned studies using a similar coating techniques and healing times. In contrast to the comparative studies, the measured BA in study II was found to be significantly higher for HA-PEEK 
compared to control surfaces. Wennerberg et al. ${ }^{240,242}$ and Sul et al. ${ }^{241}$ suggested that the HA coating at this early time point only affect the bone response at the interface, not the bone density. Since our study showed mutually increased BIC and BA, other dissimilarities between the studies must be addressed. The implant thread design and surgical protocol may affect the BA at this early time point. Wider threads may require longer healing time to be filled with bone compared to tighter threads. Further, whether the implant site is pretapped, non-tapped or if the implant is designed with self-tapping threads may affect the BA. However, within the limitations of this thesis and the comparable studies it cannot be distinguished how these properties affected the histomorphometric results. The extended healing times of 12 -and 20 weeks cannot be compared to the studies using the same coating techniques since they only evaluated the early responses.

Barkarmo et al. ${ }^{197}$ performed a pilot study of PEEK using the same spin coating technique as above. The PEEK implants were designed as a tack screw (Figure 47) and were implanted in a rabbit femoral model. In each femur, one HA-PEEK and one uncoated implant were installed. Uncoated specimens represented the control. After six weeks of healing femoral bone implants were retrieved and submitted to histomorphometric evaluations. However, 7 (4 control and 3 HA-PEEK) of the 18 implants were found non-integrated after the healing period and were therefore excluded from the study. The osseointegrated implants showed no differences between HA-coated and uncoated ones in terms of BIC and BA. Due to a large number of lost implants, the authors decided to conduct another study with identical coating but with a different implant design ${ }^{243}$. In the modified study ${ }^{243}, 78$ threaded PEEK implants were inserted into the rabbit tibia and evaluated after 6 weeks of healing. The results showed that threaded implants were preferable since all specimens were osseointegrated. The RTQ values were almost twofold and significantly higher for HA-PEEK compared to the uncoated PEEK. The quantitative histomorphometry demonstrated significantly higher percent of BIC on HA-PEEK. The authors decided to divide the BA into "all threads" and "three best ones" where only the latter group revealed significant differences in favor of HA-PEEK. When all threads were measured no differences were identified between the 
groups. On the whole, this is the most similar published study that can be compared to study I-II. Dissimilarities to this study and the ones within the actual thesis is only the time of implant retrieval. In our present study the results from the surface analyses are confirmed where the topography on the micro level was not affected after the nano HA-coating. The BA percentage showed deviant results between the studies where Barkarmo et al. ${ }^{197}$ found no differences between the groups. Several reasons may explain this outcome. One may be the location of the implant in the tibia where the region closer to the condyle possesses a thicker cortical plate compared to the diaphysis region. Other reasons could be related to the surgical protocol. The final drill diameter and dimensions of the tapping device may have an impact on the amount of bone inside the threads.

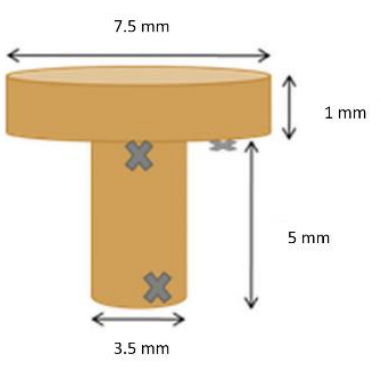

Figure 47. Design of PEEK implant used in Barkarmo et al. 2011. ${ }^{\mathrm{XI}}$

As aforementioned, results from the literature have indicated that moderately rough implant surfaces and HA-coated implant surfaces could improve the level of osseointegration. To separate these compounding effects, the implants used in this thesis were prepared with a minimally rough surface to solely attribute the effect of the applied coating.

The surface roughness and chemical appearance of PEEK will be changed after the coating procedure using HA minerals. Thus, different coating methods may result in different surface characteristics. The most common coating techniques used on commercially implants, mostly due to their FDA approval, are the plasma spray 
and electrophoretic depositions ${ }^{244}$. However, there are several weaknesses of these two techniques and alternative coating approaches have therefore been developed and evaluated.

Since PEEK is a relatively soft material, ordinary coating methods for metal implant may not always be suitable due to risk of surface abrasion and structural changes caused by the high temperatures. In this actual thesis, a dip coating method was employed to overcome these disadvantages. Dip coating deposition has been extensively evaluated on metallic implants with successful outcomes, but only a few have used the method on PEEK substrates. The surface of cementless cups in total hip replacement models on sheep were coated with HA layer using a modified dip coating ${ }^{245}$. The coating thickness was approximately $75 \mu \mathrm{m}$ according to the authors. However there were no topographic analyses presented in the published article to confirm the surface properties. Another study by the previous authors, using the same coating method, showed that HA coating onto CFR/PEEK significantly increased the interfacial shear strength in bone ${ }^{246}$. However, the implants exhibited signs of detached HA coating after a pull out test. In study II the coating adhesion was evaluated in a Sawbone material setup after implant insertion and removal. The SEM evaluation revealed a well-preserved coating, but on the thread top there were signs of HA mineral deformation. Evaluating the coating stability after insertion is a more clinically relevant evaluation than a pull out test.

The same coating method, as in this thesis, provided by Promimic AB (Göteborg, Sweden) was evaluated on PEEK and published in two in vivo studies ${ }^{197,243}$. The surface analyses using SEM and INF showed a fully covered PEEK surface by nano-sized minerals. The surface micro topography was concluded to be unchanged after the coating procedure since only small differences between HA-PEEK and PEEK were detected. This resembles to the findings from study I except that the density of summits $\left(\mathrm{S}_{\mathrm{ds}}\right)$ was significantly higher on pure PEEK. One possible reason may be that study I measured three locations of the implants (top, valley and flank) whereas Barkarmo et al. ${ }^{243}$ only analyzed the thread top. However, the AFM analysis from the aforementioned study visualized significant differences at the nano levels between HA-PEEK and PEEK ${ }^{197}$. In contrast, results from study I revealed a significantly lower developed surface ratio 
for HA-PEEK while the other parameters $\left(S_{a}\right.$ and $\left.S_{d s}\right)$ were similar to untreated PEEK. However, this confirms the conclusion from both studies that the nano HA-coating had a minor effect on the surface topography. The chemical analyses from both studies showed the stoichiometric ratio of HA. Since the surface coating showed uniformity and consistent thickness over the entire surface, the thickness of the coating could be estimated to be $5-20 \mathrm{~nm}$.

Hydroxyapatite coatings on a PEEK surface can be deposited using a sputtering technique, although, the PEEK surface need to be covered with an intermediate protective layer of Ti or YSZ. Since Ti/YSZ expand differently compared to PEEK at high temperatures (as autoclaving) a heat treatment was required to evaluate any signs of coating de-attachments. Studies have shown that a heat treatment after Ti/YSZ and HA deposition, decreases the bonding strength to the PEEK surface and delamination was found in those studies performing pull-out tests ${ }^{198,247-249}$. The produced layer of HA with this technique was amorphous which may cause a more rapid dissolution. However, the heat treatment increased the crystallinity of the HA minerals to form a denser cover over the underlying Ti/YSZ and further decrease the mineral dissolution rate. The coating thickness was not measured in any of the studies but the HA thicknesses were measured in the range from $500 \mathrm{~nm}$ up to $>3 \mu \mathrm{m}$. The surface characteristics of the HA coating used in this thesis have some advantages compared to the abovementioned studies. The heat treatment does not affect the coating stability and the thickness reduces the risk of delamination. In study I the implants were submitted to mechanical loading to monitor the strain-stress behavior of implants without heat treatment and implants exposed to $300^{\circ} \mathrm{C}$ and $350^{\circ} \mathrm{C}$. The results showed that the heat treatment involved in the coating process did not affect the elastic modulus and the peak stress of the material. One explanation for the unchanged mechanical properties is probably the short duration of the heat treatment.

An in vitro study showed that coatings with higher levels of $\mathrm{Ca}$ (i.e. higher $\mathrm{Ca} / \mathrm{P}$ ratio) were found to increase the bioactive properties of $\mathrm{HA}^{250}$. Surprisingly, none of the studies evaluated the surface topography and can therefore not be compared with the present studies. 
Consequently, since an intermediate coating layer has showed disadvantages in terms of cohesive fractures to the core material, another coating technique than plasma spray may be more suitable. Plasma spray techniques result in signs of detachment to the core material ${ }^{251}$. Plasma spray coating generates heat but by using smaller particles and extend the distance to the sample, the temperature can be kept below $\mathrm{T}_{\mathrm{g}} 143^{\circ} \mathrm{C}$ (glass transition temperature). The coating can be deposited using cold spray technique which generates a temperature of $250^{\circ} \mathrm{C}$ at the target ${ }^{252,253}$. Plasma spraying creates a coating thickness of $>100 \mu \mathrm{m}$ which is more than 5000 times thicker (20$40 \mathrm{~nm}$ ) than the coating used in this thesis. Suska et al. ${ }^{251}$ plasma sprayed PEEK with HA minerals and reshaped the surface to an average height deviation of $6.9 \mu \mathrm{m}\left(\mathrm{S}_{\mathrm{a}}\right)$. The untreated PEEK sample had $S_{a}=4.1 \mu \mathrm{m}$. According to a recognized classification system, both surfaces are categorized as rough ${ }^{221}$. In study I-IV, the spin coated implants presented a minimally rough surface, before and after the deposition of HA minerals ${ }^{254}$. The strongest bone response to an implant surface should possess a moderately rough $\left(S_{a}=1.0-2.0\right)$ micro-topography ${ }^{221}$.

The AFM outcomes and SEM images in this thesis of HA-PEEK demonstrate the coating morphology and the arrangement of nanoscale HA rods. Previous studies suggested that HA particles in the nanoscale regime enhance the osteoblast functions of alkaline $\mathrm{P}$ and $\mathrm{Ca}$ deposition ${ }^{25}$. Micro-sized HA coatings have been mainly produced by plasma spray ${ }^{256}$ and sol-gel coating ${ }^{257}$. However, for the clinical applications, the plasma spray deposition has been the most commonly used manner of coat application. However, these techniques are incapable of producing nanoscale coatings due to the high temperature which causes high amount of amorphous calcium and a mixture of grain sizes ${ }^{258}$. Nano-HA coating on titanium has been found to decrease the amount of attached cells and cell spreading on the surface compared to uncoated titanium ${ }^{259}$. However, the results showed well-developed filopodia and lamellipodia on the nano-HA surface. This may improve the bone formation and could be one explanation to the advantages of nano-coatings. Meirelles and collegues ${ }^{237}$ managed to exclusively evaluate nanotopography by removing all microstructures and apply a coating with nano features. The results showed improved bone response on the nano surface. 
However, whether the results is affected by the HA content or the nano features cannot be distinguished. The same situation appears for results in this actual thesis.

The results observed from study I on the contact angle measurements analysis showed that the HA coating improved the surface wettability. It is known that the surface wettability is affected by the surface roughness and the surface energy ${ }^{260,261}$. Hydroxyapatite is a hydrophilic material and therefore the results are expected. Further, amorphous HA has been found to be less hydrophilic compared to crystalline $\mathrm{HA}^{262}$. In the present study, all surfaces had similar surface characteristics on the micro scale and were regarded as minimally rough. However, the nano scale properties were more dissimilar. Nanotopography and the dimension of the structures are other parameters that affects the surface wettability and cellular response $^{263}$.

\section{Biomechanical properties of HA coated implants}

Removal torque measurement is a common method to evaluate the fixation of screw shaped implants in experimental studies. In addition, the stability can further be estimated by measuring the insertion torque and resonance frequency analysis (RFA). Insertion torque only provides a value for the implant primary stability and does not present detailed information on the osseointegration. RFA, on the other hand, can be measured at the implant placement and during the healing period to estimate the interfacial stiffness and be the basis for decisions in loading protocols ${ }^{264,265}$. However, the ultimate strength of the bone-implant connection can be better measured by removal torque ${ }^{262}$. Removal torque of a cylindrical implant without threads is referred as pull-out test. There is an ongoing debate whether RTQ or pull-out tests reflects the clinical failure of an implant. Christensen et $\mathrm{al}^{266}$. found no linear correlation between RTQ and pull-out test while some researchers claim the unscrewing not being a failure mode seen in the clinical reality ${ }^{267}$. However, due to the few number of published studies on RTQ and PEEK, the subsequent discussion will compare and relate RTQ results to pull-out tests.

In this thesis, the biomechanical properties of test and controls were compared in a well-established animal model. By evaluating 
the outcomes of HA-PEEK compared to PEEK in an experimental split design, the RTQ values can be statistically compared in a pairwise fashion. In study I and IV the removal torque was evaluated after 3, 12 and 20 weeks of healing. The HA coating showed a positive effect on the retention of the implant after all healing times. Even though a comparison of absolute values is impossible, there is a trend showing less retention after 12 weeks compared to 3 weeks. Further, after 20 weeks of healing the required removal torque had a tendency to be lower than after 3 and 12 weeks. The reasons for this finding can only be speculated. The results may reflect the natural process of bone apposition and bone remodeling. The biomechanical characteristics is gradually transformed from being a primary stability due to the threaded implant design to a retention based on bone apposition. The removal torque curve is likely to be determined by the quality of the surrounding bone in terms of stiffness and density. In addition, it may be speculated that the amount and effect of the HA after 12 weeks was inferior to the 3 week time point. Studies concluded the effect of HA to be prominent during the first healing period of osseointegration when immobilization of osteogenic cells and formation of vascularized bone on the implant surface occurs ${ }^{268}$.

The tendency towards gradually sinking RTQ over time was opposing biomechanical results from other studies evaluating HA coating on titanium and PEEK where the retention increased with time ${ }^{246,269,270}$. Furthermore, titanium implants without coating showed gradually increased its RTQ values with time after implantation $^{271}$. The RTQ values were found dependent on the implant surface structure, implant design, adjacent bone structure and the amount of bone area next to the implant. This study may be used as a reference marker to other studies with healing time as the only variable. Regardless of the coating material, there are some studies demonstrating similar results when evaluating RTQ after different healing times ${ }^{272,273}$. The final conclusions resemble these of this thesis.

In the present study, the underlying texture of the pure PEEK was minimally rough and the surface micro-topography remained nearly unchanged after the coating procedure. This resembles surface properties of HA-titanium implants evaluated by Meirelles et al..$^{236,237}$ and 
Jimbo et al. ${ }^{238}$. When the results from the cited studies are taken in consideration, it appears that the underlying topography has a major impact on the RTQ values. Thus, if the underlying surface roughness holds an optimal topography according to Albrektsson and Wennerberg $^{221}$, the coating does not improve the RTQ values. However, when the underlying micro-topography is minimally rough, as in study I, the RTQ values become higher for the HA-coated implants.

Since the surface topography on the micro- and nano levels was approximately identical for PEEK and HA-PEEK the discussion continues to the dissimilarities in the chemical composition. The results from the XPS showed the presence of $\mathrm{CaP}$ on the surface of the coated implants with close to the theoretical stoichiometric ratio. Difference in $\mathrm{CaP}$ ratios have been found in vitro to affect the rate of osteoblast proliferation ${ }^{274}$. Jimbo et al. ${ }^{238}$ found an influence of $\mathrm{CaP}$ chemistry on the biological outcomes in terms of RTQ and histomorphometry. Meirelles and co-workers ${ }^{236}$ produced nanotopography using $\mathrm{HA}$ as coating material which showed higher retention in rabbit bone after 4 weeks of healing.

It is always difficult to compare results from one in vivo study to another due to the diverse conditions as experimental site, implant design, type of animal and surface properties. However, the biomechanical results from this study showed significantly increased RTQ for HA-PEEK compared to untreated PEEK. Therefore, it is suggested that HA modified PEEK implants may shorten the time of loading and shorten the healing time in bone with impaired quality.

\section{Bone formation around PEEK and HA-PEEK}

As mentioned earlier, PEEK possess a hydrophobic surface due the aromatic ring and polyester functional groups in its chemical structure which reduce protein and cell adhesion ${ }^{275,276}$. Hydroxyapatite is more hydrophilic than PEEK because of a hydroxyl group in its structure which provides a more favorable surface for cell growth and protein attachment ${ }^{277}$. Hydroxyapatite enhances the integrinmediated adhesion of the surrounding cells which contributes to the ability to bind directly to bone. Increased cell proliferation has been demonstrated around HA-coated PEEK in a cell adhesion array ${ }^{195}$. Fibronectin, a cell adhesion protein, was localized around the cells at the surface of HA-coated PEEK. This was one of the findings to 
draw the conclusion that HA-PEEK is more bioactive than pure PEEK $^{277,278}$. This hypothesis can be confirmed from the result in this thesis.

The effect of crystalline HA deposited on PEEK was evaluated after 3,12 and 20 weeks of healing in an experimental rabbit tibia and femur model. Prominent observations were found after the early healing time where HA-PEEK, in comparison to controls showed considerably higher grade of osseointegration in tibia with respect to BIC and BA (Study II). In femur after the same healing time, HAPEEK revealed significantly higher BIC compared to untreated PEEK (Study III). However, the BA inside the threads of HA-PEEK showed no difference between the two groups.

The results were as expected and may be explained using logical reasoning with previous knowledge from similar studies and the biology of bone. After 3 weeks, some amounts of the former bone were still remaining around the implants. Therefore, no difference in BA $(\mathrm{P}=0.5)$ inside the femoral threads was distinguished where the whole length of the implant was surrounded by trabecular bone. In addition, the implant site was tapped prior to implant placement which, at this time point, would additionally increase the amount of old bone inside the threads. Moreover, the turnover rate of the trabecular bone is higher than that of the cortical which further accelerates the bone growth into the threads, more rapidly in the tibia bone marrow, possibly due the absence of a foregoing bone resorption $^{278}$. In the tibial bone where the majority of the implant length was inside the bone marrow there was a significant difference in BA. The variance was identified in the bone marrow where the HA-PEEK showed osteoconductive properties as the bone grew along the implant side.

The BIC after 3 weeks was significantly higher for HA-PEEK, compared to pure PEEK in tibia and femur. However, after 12 weeks of healing, there was a significant difference in the femur but not in the tibia. Potential reasons for this outcome may involve the higher bone turnover in femur due to the trabecular bone. According to Wolff's law ${ }^{40}$ the bone will adapt to the applied load and even though there was no external load on the implants there is a tension on the threads. This tension might stimulate bone formation even after 12 weeks of healing in the femur. Further, the ductility of the 
trabecular bone may distribute the stress more evenly to the surrounding structures. In contrast, in tibia there were no difference in BIC after 12 weeks. No signs of fibrous tissue was seen on the histological sections and the percent of bone in contact with the implants was not increased for the HA-PEEK. The uncoated PEEK increased its BIC more if compared to the HA-PEEK from 3 to 12 weeks. Obviously the bioactive effect of HA was no longer improving the bone formation. This occurrence has been detected by other authors. Studies have concluded that a smooth $(\mathrm{Sa} \leq 0.2 \mu \mathrm{m})$ underlying surface of the implant shows lower BIC after a longer healing time compared to rougher surfaces ${ }^{247,279}$. However, using a smooth surface enables a more exclusive evaluation of the coating. A study by Durham et al. ${ }^{247}$ evaluated PEEK with HA/YSZ coating in rabbit femur after 6 and 18 weeks of healing. The BIC of all groups were less after 18 weeks compared to 6 weeks. In addition, the difference between PEEK and HA/YSZ-PEEK was larger at 18 week. The BIC in this actual study did not significantly improve from 3 to 12 weeks but in contrast there was a significant difference between the groups after both healing time points. The author concluded the decrease in BIC to be caused by the smooth appearance of the implant. Increased BIC has been demonstrated on several studies of PEEK where the conclusions were drawn that the HA possesses a chemical effect on the bone formation which improved the osteoblastic differentiation and bone growth ${ }^{243,251-253,280}$. Many reports have confirmed that HA coating on titanium implants show increased $\mathrm{BIC}^{270,281}$. However, to the knowledge of the authors, it has not been clarified whether this effect is caused by the surface topography, by altered chemical appearance or whether these properties act synergistically.

In the present study, the raw values of BIC and BA were rather low if the implant are to be exposed to any stress or strain. The histological definition of osseointegration is a "direct anchorage of an implant by formation of bony tissue around the implants without the growth of fibrous tissue at the bone-implant interface" ${ }^{282}$. Bone formation around commercial titanium implants normally obtain approximately $50 \%$ of BIC in rabbit bone after 4-6 weeks of healing. ${ }^{283-285}$. These low values of BIC on PEEK and HA-PEEK in rabbit 
agree with some previous studies and oppose some others. Some authors demonstrated levels of BIC in the same region as this study ${ }^{197}$, and others show values with comparable level as titanium implants $^{243,247,251}$. All of these studies, including ours, are difficult to be compared due to the various regions of interest and implant geometry.

As mentioned before, one major aspect of a successful osseointegration is the surface wettability which was for PEEK hydrophobic and hydrophilic for HA-PEEK. The results and the present literature indicate that the hydrophobic and minimally rough surface of PEEK will affect the absolute levels of BIC. An in vivo study in rats has demonstrated that hydrophobic implants compared to hydrophilic ones showed reduced cellular response in the early healing stages ${ }^{286}$. However, after 3 weeks the study revealed no substantial difference between hydrophobic and hydrophilic implants. The hydrophilicity affects the total amount of proteins bonded to a surface and their bonding strength. In addition, the composition of the protein film attached to the surface is determined by the hydrophilicity ${ }^{287,288}$. The macromolecular film will alter the surface charge and wettability from the original implant surface. Thus, the hydrophilicity affects the cellular response via the formation of a protein film. The wettability of the implants in the present study was measured by the contact angle of a droplet. Hydroxyapatite coated PEEK revealed a good wetting as the contact angle was $53^{\circ}$ which was $35^{\circ}$ less than the unmodified PEEK. The unmodified PEEK showed an incomplete wetting $\left(88^{\circ}\right)$ and the surface energy was decreased compared to HA-PEEK. If the wettability alone has an impact on the results in this study cannot be narrated within the limitation of this study. Whether a hydrophilic surface is more optimal for osseointegration remains unknown. However, the literature present evidence that the surface has an obvious impact on the biological and clinical outcomes $^{289}$.

Qualitative observations of the histological sections were carried out using an optical light microscope. In study II we used Masson Goldner-Trichrome staining system because of its ability to differentiate hard tissues from soft tissues and fat. Due to the disadvantage of Masson Goldner-Trichrome staining in separation of new and old bone, the following studies used the conventional Toluidine Blue 
staining. There were some difficulties with the grinding process of the PEEK samples due to the relatively porous property of PEEK. Therefore the qualitative analysis of the histological sections was to a certain extent problematic to interpret. Osteoblast and giant cells were difficult to detect on the sections. However, the formation and extension of the new bone in close proximity to the implant surface can clearly be viewed into the tibial bone marrow. Osteoid sectors were found around the implant but it was difficult to detect attendant osteoblasts. Osteocytes were more visible, probably due to the level of coloration from the staining. There were not any widespread formations of fibrous encapsulation around HA-PEEK surfaces. However, on the neat PEEK there were areas of fibrous tissue in proximity to the implant surface, as reported in other published studies ${ }^{10,290,291}$. Some studies have located spreading giant cells next to the implant surface which could be an indication of a foreign body reaction ${ }^{243}$. However, this finding was not confirmed by results from the present study.

The implants installed in the femur were designed with a perforating hole in the apical part. PEEK is today a popular and successful alternative to metal in spinal surgery where the main objective of the implant is to allow a bone fusion within the vertebras ${ }^{292,293}$. Therefore. PEEK implants with a perforating hole was selected and applied in the femur where the implant turned out to be fully covered in bone. The BA after 3 weeks and 12 weeks was presented in study III and the results were very convincing. The area of bone inside the HA-PEEK after 3 weeks was almost 4 times greater that PEEK $(\mathrm{P}=0.00)$. After 3 weeks the bone nearly managed to meet the opposing bone in the middle of the implant perforation. After 12 weeks the BA was still significant in favor for HA-PEEK but the difference was leveled out to a 2 -fold increase. The trabecular spreading continued inside the perforations as outside the implant and approached the implant surface with frequent intervals. The line between new and old bone could be distinguished at the entrance of the perforation (Figure 48). 


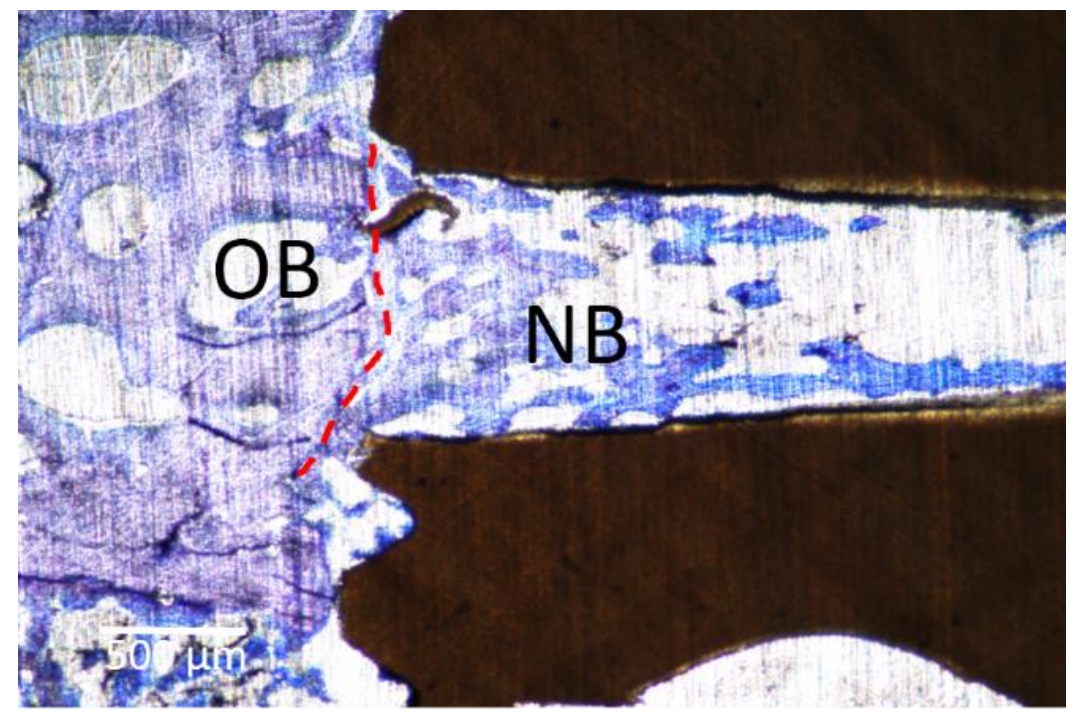

Figure 48. Histological section of an HA-PEEK after 3 weeks of submerged, unloaded healing. (OB): Old bone, (NB): New bone. Scale bar $=500 \mu \mathrm{m}$

As far as known by the author, there are no similar studies that can be related with the results from this study. However, there are some clinical and experimental radiographic studies that evaluated the level of bone fusion using PEEK as implanted material. One major difference to the comparative studies is that the implants in the present study was not exposed to any physical load during the healing time. Walsh et al. ${ }^{290}$ evaluated the bone formation in a sheep cervical fusion model. The benefit of HA incorporated into PEEK was visible in the micro-CT and histological evaluations and showed direct bone contact with the implant surface, without interviewing fibrous tissue. The bone inside the fusion chamber showed a higher level of maturation for the HA-PEEK group compared to unmodified PEEK implants. A retrospective and case-control study of 124 patients which underwent TLIF using cages (Figure 49) of nanoHA/PA66 (nanohydroxyapatite/polyamide66) and PEEK alone evaluated the radiological changes after an average follow up of 2 years ${ }^{180}$. The radiographic outcomes of the HA-modified PA66-cages revealed comparable results as pure PEEK in terms of fusion rate and subsidence. Durham and co-workers ${ }^{247}$ showed increased bone fusion and 
BIC on HA-PEEK in a rabbit femur model. Further, the fusion volume increased significantly from 6 weeks to 18 weeks. However, the fusion perforation was parallel to the axis of the implant. Therefore, the bone fusion could only start from the side facing inwards. However, it still remains unknown whether the amount and rate of fusion have a relationship with clinical success ${ }^{293}$.
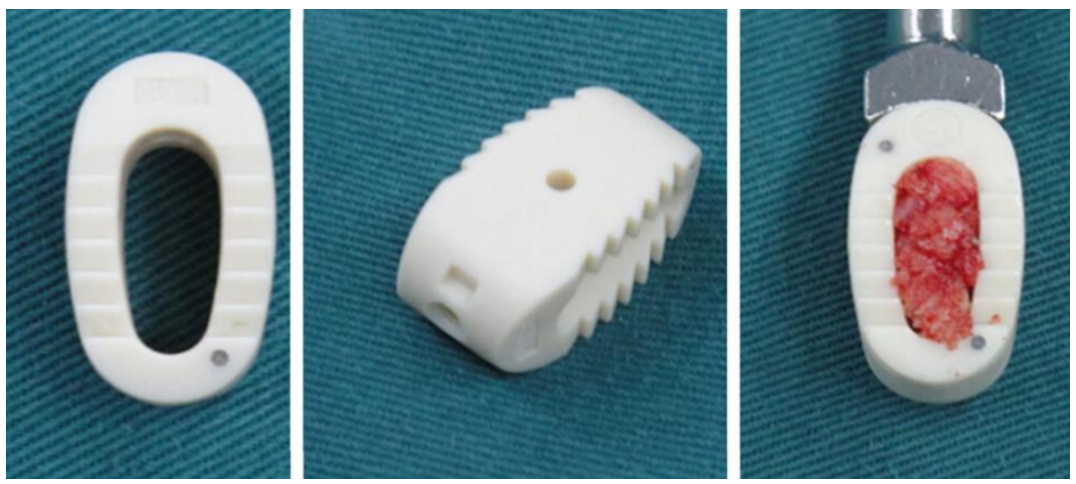

Figure 49. PEEK cages modified with nano-HA/PA66 in lateral and superior views.

Since the BIC percentage after 12 weeks was of comparable percentage to that of 3 weeks, we decided to perform an evaluation after an extended evaluation period of 20 weeks. The bone remodeling period of a rabbit is almost 3 times faster than the rate of a human. Correspondingly, 20 weeks of healing in a rabbit bone is equivalent to more than a year in a human bone which it sometimes requires to achieve a fully regenerated bone structure ${ }^{294}$. At this time there is an ongoing remodeling from immature bone to a mature lamellar bone. This bone remodeling is a continuous process which is carried out throughout the life of the presence of the implant.

After 20 weeks of healing there were no statistical significantly differences in BIC or BA between HA-PEEK and PEEK. However, $\mathrm{BIC}$ and $\mathrm{BA}$ in the femur were higher for HA-PEEK. The per cent of $\mathrm{BIC}$ in the femur was around 20 which is rather low compared to titanium implants after an extended healing time ${ }^{295}$. However, the BIC of the top three threads showed a more prominent none attachment for HA-PEEK, if not statistically significant. The general conclusion from the 20 weeks experiment was that the effect of the HA 
was no longer visible at the implant surface. However, since the RTQ was significantly increased we hypothesize that the bone quality around the implants has been improved. Even though RTQ may not be a natural test in the clinical situation, it provides a more comprehensive image of the biological event.

An even more comprehensive and detailed image can be obtained using computed tomography. The technique is non-destructive and the researcher does not need the highly advanced skills in specimen preparation and microscopy ${ }^{296}$. In addition to measurements of bone volume, the morphology, structure and density of the newly formed bone can be assessed. Polyetheretherketone is an optimal material for micro-CT evaluation since it does not create any metal streak artifacts ${ }^{10}$. In study II, three different volumes around the implants were evaluated in terms of bone volume density (BV/TV), specific bone surface (BS/BV), trabecular thickness (Tb.Th), number (Tb.N) and spacing (Th.Sp). The radiographic outcomes only provided statistical significant difference in $\mathrm{BS} / \mathrm{BV}$ and $\mathrm{Tb}$. Th with a contradictory logic. The specific bone surface was larger for PEEK and the trabecular thickness around HA-PEEK was larger. Since the number of trabecular was similar in the two groups, the bone surface should be proportional to the trabecular thickness. This data was from the inner VOI where the threads were included which might have affected the radiographic outcomes. Comparative results from 3 to 12 weeks demonstrated that the bone remodeling was more prominent in vicinity to the implant surface.

The results from the RTQ was theorized to have an association to the quality and density of the surrounding bone. However, the micro-CT results showed similar bone formation around the implants after all healing times. Yet, the bone hardness and level of mineralization are not detected on the micro-CT which might be were a possible cause to the increased RTQ values. Bone hardness can be measured using nano-indentation ${ }^{239}$. Although, nano-indentation could not be applied in this study due to limitations of the currently used embedding techniques, several studies have conducted micro-CT evaluations of HA-modified PEEK. Some studies showed similar results as the current ones and the common factor is that only the chemical properties were changed between the groups, not the sur- 
face topography ${ }^{247,297}$. Other studies showed a more obvious increase in bone formation around HA-PEEK implants compared to unmodified PEEK ${ }^{252,253,298}$. Further, some studies showed an altered trabecular architecture which the author suggest to be attributed to the osteoconduction and osteoinduction of the surface ${ }^{252}$.

Computed tomography was as well performed on the implants retrieved after 20 weeks of healing. There was a higher mean value of BV/TV from the entrance to $400 \mu \mathrm{m}$ for the HA-PEEK. However, the difference was not statistically significant possibly due to the few number of samples. Moreover, it was difficult to segment the different structures due to low contrast between the PEEK material and the resin, which the samples were embedded in. With a more clear contrast, the segmentation and identification of structures becomes more difficult. For the future the segmentation could be improved by changing the absorption coefficient of the resin to be different from PEEK. The overall conclusion from the micro-CT evaluation was that both HA-PEEK and PEEK generated a healthy bone response and development.

\section{Genetic mechanisms}

There are several techniques to determine if osseointegration has occurred such as RTQ, computed tomography and histology. However, to fully understand the underlying mechanisms of bone formation, the researcher needs to assess the cell signaling of cytokines. In this thesis the gene expression was performed on implants that were removed during the RTQ evaluation. Most studies on gene expression and PEEK are using commercial mesenchymal stem cells. However, the extraction of RNA from removed implants provide a stronger correlation to the other results reported in this thesis. After 3 weeks there was a significant up-regulation of BMP-2, Col1A1 and IGF1 around HA-PEEK. BMP-2 is involved in the differentiation of mesenchymal stem cells to chondroblasts and osteoblasts ${ }^{299-301}$. BMP-2 has been shown to increase the quality of the reparative tissue after a bone or cartilage defect ${ }^{302}$. The BMP-2 was expressed more than three times on HA-PEEK compared to the control which could explain the significantly higher BIC. A study by Wang et al. ${ }^{303}$ revealed an up-regulation of BMP-2 of the cells on unmodified PEEK compared to tissue culture plate (TCP). No logical explanation can 
be applied to explain any correlation between these results and our results.

The second up-regulated gene, Col1A1, plays a major role in encoding type I collagen, which are found in most connective tissues. Mutations or disorders associated with Col1A1 are found in several medical conditions such as osteogenesis imperfecta, Paget's disease and osteoporosis ${ }^{304}$. Moreover, the alignment and type of collagen will change the amount and disposition of the bone minerals which might affect the mechanical property ${ }^{305,306}$. The threefold up-regulation of Col1A1 may be indicative of an increased mechanical properties of the bone. This might explain the increased RTQ values at 3 weeks for HA-PEEK. However, the strength of the bone depends whether the fibers are arranged longitudinally or transversally to the osteons ${ }^{307}$. The interactions between the collagen fibers and the mechanical properties of bone is a complex interrelationship and is today not fully clarified.

The third gene that was significantly expressed in HA-PEEK was IGF1. This gene is a polypeptide that has an anabolic effect and plays a major role during childhood growth and development ${ }^{308}$. Moreover, IGF1 is a mitogen factor for osteoblasts and has a major impact on bone formation, particularly the periosteal growth ${ }^{309}$. IGF1 was twofold up-regulated in HA-PEEK compared to PEEK with a high level of statistical significance $(\mathrm{P}=0.0008)$.

Accordingly, all the genes that were significantly up-regulated in HA-PEEK are involved in osteoblast differentiation and bone formation. However, there are some tested genes that surprisingly were unchanged. In osteoblastic differentiation, RUNX2 is a key transcription factor. RUNX2 knockout mice is born with impaired mineralization in the skeletal regions of endochondral ossification ${ }^{310}$. Unchanged RUNX2 levels were confirmed by Zhao et al. ${ }^{188}$ who compared PEEK and nano-structured PEEK. Additionally, a downregulation of RUNX2 was demonstrated in cells incubated in the presence of nano-HA ${ }^{311}$. SPP1 is involved in osteoblast attachment to hydroxyapatite in bone and is normally expressed in higher levels at later stages of mineralization ${ }^{312,313}$. Since the levels of HA is considerably higher in HA-PEEK, SPP1 was expected to be significantly up-regulated. One possible explanation to the unchanged expression 
could be that SPP1 will be up-regulated later than 3 weeks. However, He et al. ${ }^{312}$ showed an up-regulation of RUNX2 and SPP1 after 3 weeks in HA-coated silk scaffolds. The BGLAP gene, or osteocalcin, is a bone protein that is secreted by osteoblasts to regulate the bone remodeling. In this study, the levels of BGLAP were unchanged in HA-PEEK. Contradictory, a study showed up-regulation of BGLAP after 2 and 3 weeks in YSZ/HA-PEEK implants ${ }^{249}$. The expression of BGLAP is dependent on signaling from RUNX2 $2^{314}$. Therefore, the results of unchanged regulation of RUNX2 and BGLAP are considered to be correctly interpreted.

Gene expression analysis using RT-PCR is an advanced and valuable technique to amplify a certain DNA sequence, but it comes with some limitations. Due to the sensitivity there is always a risk to contaminate the sample by traces from foreign $\mathrm{DNA}^{315}$. Moreover, the technique only identifies the presence or absence of a gene at a certain time point (Real Time). Therefore, the outcomes can only be scored against the reference gene and against the control sample. A new Droplet Digital PCR has been introduced which decrease the biases and can provide an absolute count of the target $\mathrm{DNA}^{316}$.

In evaluations of the qualities and performance of a new biomaterial for clinical use, the FDA requires extensive in vitro and in vivo tests. In vitro testing is normally the first stage test to determine toxicity and cytocompatibility before the experimental animal testing. Moreover, in vitro studies provide information on cell proliferation and differentiation, cytotoxicity and genotoxicity ${ }^{317}$. An advantage is that the test can be standardized and the outcomes are easy to measure and compare ${ }^{318}$. However, in vitro testing can only demonstrate the response to a material from a cell line or tissue fragments, not from vital tissue as in vivo testing is capable of. In contrast, experimental animal models can evaluate the tissue response with or without loading conditions, in healthy or compromised conditions and with animals of different ages. Animal models are relatively close to humans in terms of mechanical and physiological conditions but the outcomes are not entirely authentic.

In this thesis the in vivo testing were performed on rabbits due to several reasons. Primarily, the current research group has evaluated 
implants in rabbits with the same study model earlier, with successful statistical power and results. In terms of cost-effectiveness and number of implants, rabbits meets the requirements to the present study since there were only two implant groups. However, the rabbits are very different from humans with regards to bone microstructure and bone remodeling ${ }^{214}$. Compared to the human secondary bone structure, the rabbits have more longitudinal tissue structure and canals of osteons parallel to the long axis of the bone ${ }^{319}$. The rabbits have a faster bone remodeling than humans (approximately x 2.8), especially the Haversian remodeling ${ }^{215,320}$. Therefore, the results are difficult to directly compare with humans. However, the rabbit is adequate for demonstrating the bone response to different types of implant materials. 


\section{CONCLUSION AND FUTURE PER- SPECTIVES}

In the present research the bioactive properties of hydroxyapatite modified PEEK was investigated and from Studies I-IV it can be concluded that:

1. Polyetheretherketone showed no significant decrease in stress-strain performance after a heat treatment of $300^{\circ} \mathrm{C}$ and $350^{\circ} \mathrm{C}$ (Study I). This verifies that the mechanical properties are maintained after the coating procedure and subsequent steam autoclaving. The application of nanosized HA coating on PEEK only alters the density of summits on the microscale level (Study I). PEEK and HA-PEEK exhibits a minimally rough surface which is too smooth for optimal osseointegration. The coating procedure was successful by means of generating a full coating coverage of the surface without signs of contamination (XPS in Study I).

2. The adhesion stability test showed that the majority of the coating remained at the surface after insertion of a HA-PEEK implant into a solid polyurethane foam (Study II). Moreover, the insertion torque was similar of HA-PEEK and neat PEEK. This finding is of major importance and ensures a complete effect of the bioactive coating in situ.

3. A nanoscale HA-coating on PEEK significantly increased the required force to remove the implants by rotational measure (Study I and IV). However, the absolute required force was decreasing with time which might indicate a dissolution of the coating or partial fibrous encapsulation. 
4. The crystalline HA-PEEK implants placed in the tibia showed after 3 weeks significantly higher BIC and BA compared to unmodified PEEK (Study II). After 12 weeks of healing the BIC was significant improved in femur and tibia around HA-PEEK implants in comparison with uncoated PEEK (Study II-III). The most striking outcome was published in Study III with a significantly higher BA inside the apical perforation of HA-PEEK implants. Evaluation after 20 weeks showed no differences between HA-PEEK and PEEK (Study IV). The authors suggested that the effect of the coating is not present after 20 weeks and the osseointegration relies on the underlying surface properties.

PEEK is today a popular and successful alternative to metallic implants in spine surgery, hip joint replacement and maxillofacial surgery. In dentistry, PEEK is not used as an implant but instead as a load bearing construction and temporary healing abutment. Still there are some disadvantages with PEEK as an implantable biomaterial with respect to its bioinertness and hydrophobic appearance. This project demonstrated a novel technique to modify the PEEK implant surface and alter its chemical and wetting properties.

On the basis of i) bone area regenerated in the threads and apical perforation, ii) trends of change in BIC over time and iii) the biomechanical interfacial shear stress required for implant removal, the HA coated PEEK implants showed improved bone response compared to PEEK alone in these fundamental characteristics of osseointegration. The nano crystalline HA surface, created by the spin-coating procedure provided a more favorable surface for persistent bone apposition and growth in the early healing of rabbit bone, compared to unmodified PEEK. The implant design with a perforation hole in the apical section is unique of its kind and ensures an undisturbed evaluation of bone fusion without coating abrasion or adverse loading.

This thesis provide evidence showing that the bioactivity of PEEK can be improved using nano-HA coating. However, the current modification only improved the osseointegration in the early stages 
of healing. A dental and loadbearing orthopedic implant requires a continuous increase of bone formation and osseointegration. Further research should address to extend the effect of the coating and improve the underlying surface of PEEK. That could be done by combine several techniques of making PEEK more bioactive. Incorporation of nano-HA into PEEK, blast or etch the surface and finally spin coat a liquid nano-HA solution could all improve the bioactivity considerably. With this approach, the nano-HA will be present at the surface, retained in the pits and pores, and retained inside the core material. This combination might create an implant with a bioactive effect during the complete progression of osseointegration.

Another aspect of great importance is the behavior of modified PEEK implants during loading conditions. Most of the current literature evaluate PEEK after submerged healing. The future research should evaluate PEEK in a situation more similar to the clinical reality. Furthermore, the location of the implant should correspond the final application. Therefore, loaded conditions in the mandible/maxilla or intervertebral space should be considered in the future experimental protocols. 


\section{POPULÄRVETENSKAPLIG SAMMAN- FATTNING}

Idag lever människan längre än någonsin förut, och antalet äldre kommer exponentiellt öka de nästkommande åren. En åldrande befolkning innebär stora utmaningar för sjukvården på grund av ökat slitage och kroniska sjukdomar på skelett, leder och tänder. I och med det, kommer allt fler patienter behöva behandling med tandoch benimplantat för att återställa förlorad funktion och utseende.

Titan är det vanligaste implantatmaterial som används inom tandvård och till viss del inom ortopedi, på grund av dess goda biologiska och mekaniska egenskaper. Titan har dock uppvisat en del brister, bland annat på grund av dess avvikande styvhet till kroppens skelett. Ett alltför styvt implantat kan ge upphov till ogynnsam kraftfördelning som vid belastning kan leda till destruktion av kringliggande hårdvävnad. Förutom detta har enstaka fall av titanallergi dokumenterats och i avsikt att möta patienters önskemål om metall-fri behandling med naturfärgade implantat har alternativa material utvärderats.

Polyetereterketone (PEEK) introducerades inom ortopedin i slutet av 90-talet för att överkomma de nackdelar som metaller uppvisat. PEEK har en elasticitets modul som är jämförbar med kortikalt ben vilket, vid belastning, medför en gynnsam kraftfördelning till närliggande vävnader. Dessutom är PEEK biokompatibelt och bioinert vilket gör att den inte orsakar någon allergisk, toxisk eller mutagen reaktion vid kontakt med kroppens vävnader.

Å andra sidan skapar PEEK's inerta och hydrofobiska yta en oförmåga att binda till sig protein och celler till dess yta. Därmed sker ingen osseointegration, direktkontakt mellan materialet och levande ben, istället bildas oftast en bindvävskapsel runt PEEK implantatet. 
Sammanfattningsvis visar resultaten från denna avhandling att den bioinerta ytan på PEEK kan göras bioaktiv och därmed öka osseointegrationen med hjälp av en ytbehandling med nano-partiklar av hydroxylapatit (HA). Effekten visade sig vara mest tydlig i den tidiga inläkningsfasen. Denna ytmodifiering kan användas på implantat i situationer med undermålig benkvalitet eller då en snabb beninläkning är nödvändig.

Avhandlingen är uppdelad i 4 delstudier som utgörs av en omfattande kemisk och topografisk analys samt olika metoder att utvärdera osseointegration.

Det som gör denna avhandling unik är kombinationen av en modern polymer, PEEK, och en innovativ ytbehandlingsteknik av bioaktivt HA.

De experimentella försöken utförs på kaniner där obehandlade PEEK och HA-belagda PEEK skruvar implanteras i lårbenet och smalbenet. De implantat som placerades i lårbenet är designade med ett genomgående hål för att kunna utvärdera benformation in i ett tomrum där ben inte tidigare fanns. Efter 3, 12 och 20 veckors läkning, avlägsnas implantaten och utvärderas med urvridningstest, mikroskopering och 3D-röntgen.

I studie I och IV visar resultaten från urvridningstesterna en signifikant ökad retention för HA-PEEK vid samtliga läkningstider. Dock är urvridningsmotståndet relativt lågt, jämfört med titan, och sjunker vid 12 och 20 veckors utvärdering i förhållande till 3 veckor.

Benformation kan utvärderas under mikroskop genom att mäta procentuell implantatkontakt med ben och arean av ben mellan skruvgängorna. Resultaten presenteras i studie II, III och IV och kan sammanfattas med en statistiskt säkerställd effekt av HA på benformationen vid implantatytan efter 3 veckors läkning. Det mest sensationella resultatet påvisade de implantat som utvärderades i lårbenet. Efter 3 veckor var det nästan 4 gånger mer ben-area vid HA-PEEK än obehandlad PEEK. Vid 12 veckor var skillnaden reducerad men ändå cirka dubbelt så stor för HA-PEEK. 


\section{ACKNOWLEDGMENTS}

Completing this $\mathrm{PhD}$-project has been a period of intense learning, not only in the scientific area, but also on a personal level. It has been a great privilege to work with a team of highest rank in the field of implantology. The success and final outcome of this thesis required a lot of guidance and assistance from many people. Therefore, I would like to express my deepest gratitude to:

Professor Ann Wennerberg, my supervisor and mentor. You inspired me during the master thesis to continue research in the field. I am today, endless gratitude for that, and for all the support and guidance you have provided during these 5 years. I am looking forward to future inspirations and projects.

Dr. Ryo Jimbo, my co-supervisor. Thank you for all the scientific discussions and support. I would also like to thank you for having me as a guest in Japan. It was a great scientific experience filled with unforgettable memories. / Pär-san

Dr. Per Kjellin, my co-supervisor and CTO in Promimic AB. Thank you, and all the Promimic staff, for providing numerous surface analysis and all the precious discussions on the project design and outcomes.

Professor Tomas Albrektsson for being a role model to researchers and source of inspiration. I would also like thank you for all the proof-reading of my articles and thesis.

Dr. Silvia Galli, colleague and friend. You are a true source of inspiration and I am so impressed by all your achievements. Thank you for the assistance you have provided during the years and all the great times during dinners and gatherings. Looking forward to more of that in the future. 
Dr. Bruno Chrcanovic, colleague and friend. Thank you so much for all the assistance in the lab and especially helping out with the statistics.

To Ali Alenezi and Ricardo Trindade, fellow PhD-students and friends. I will never forget the great time in Gothenburg during the material course and for all the memorable moments we shared over the years. This is only the beginning of a life-long friendship and collaboration.

To all the Japanese and Italian colleagues and friends, Dr. Francesca Cecchinato, Marco Toia, Michele Stocchero and Dr. Yoshihito Naito and Dr. Yohei Jinno. For the great time together at the scientific courses and conferences and especially the help you provided on my project.

To the research collaborators, Sargon Barkarmo, Niccolò Peruzzi and Mohammed Hawthan. Thank you providing support and help of your expertise on our shared projects.

And finally, last but by no means least. My beloved partner in life, Kristin. Thank you for you unconditionally love and support during these intense years. During the last and toughest period I have had the best motivation ever, knowing that we will have a daughter a few weeks before the dissertation. Now, we share a lifelong project and I will always support and love you. 


\section{REFERENCES}

1. Williams DF. The Williams Dictionary of Biomaterials. Liverpool University Press; 1999.

2. Donaruma LG. Definitions in biomaterials, D. F. Williams, Ed., Elsevier, Amsterdam, 1987, 72 pp. Journal of Polymer Science Part C: Polymer Letters. 1988;26(9):414-414.

3. Heyn M. Zur Wiederverwendung maschinenlesbarer Wörterbücher : eine computergestuitzte metalexikographische Studie am Beispiel der elektronischen Edition des "Oxford advanced learner's dictionary of current English". Tubingen: M. Niemeyer; 1992.

4. Williams DF. On the nature of biomaterials. Biomaterials. Oct 2009;30(30):5897-5909.

5. Ratner B, Hoffman A, Schoen F, Lemons J. Biomaterials Science - An Introduction to Materials in Medicine. 3rd ed: Elsevier - Academic Press; 2013.

6. Global Orthopedic Biomaterials Market. India: ReportsnReports.com;2016.

7. Dudley J. Implants for the ageing population. Australian dental journal. Mar 2015;60 Suppl 1:28-43.

8. Biomaterials Market by Type of Materials - Global Forecast to 2021. marketsandmarkets.com;2016. BT 1556.

9. Coleman MM, Painter PC. Fundamentals of Polymer Science: An Introductory Text, Second Edition. Taylor \& Francis; 1998.

10. Kurtz SM, Devine JN. PEEK biomaterials in trauma, orthopedic, and spinal implants. Biomaterials. Nov 2007;28(32):4845-4869.

11. Morawetz H. Polymers: The Origins and Growth of a Science. Dover Publications; 2002.

12. Craver C, Carraher C. Applied Polymer Science: 21st Century. Elsevier Science; 2000.

13. Goodman SH. Handbook of thermoset plastics. Noyes Publications; 1986.

14. Carraher CE. Seymour/Carraher's Polymer Chemistry: Sixth 
Edition. CRC Press; 2003.

15. Muffly TM, Tizzano AP, Walters MD. The history and evolution of sutures in pelvic surgery. Journal of the Royal Society of Medicine. Mar 2011;104(3):107-112.

16. Shastri VP, Lendlein A. Materials in regenerative medicine. Advanced materials. Sep 04 2009;21(32-33):3231-3234.

17. Taghizadeh B, Taranejoo S, Monemian SA, et al. Classification of stimuli-responsive polymers as anticancer drug delivery systems. Drug delivery. Feb 2015;22(2):145-155.

18. Asghari F, Samiei M, Adibkia K, Akbarzadeh A, Davaran S. Biodegradable and biocompatible polymers for tissue engineering application: a review. Artificial cells, nanomedicine, and biotechnology. Mar 2017;45(2):185-192.

19. Najeeb S, Zafar MS, Khurshid Z, Siddiqui F. Applications of polyetheretherketone (PEEK) in oral implantology and prosthodontics. Journal of prosthodontic research. Jan 2016;60(1):12-19.

20. Panayotov IV, Orti V, Cuisinier F, Yachouh J. Polyetheretherketone (PEEK) for medical applications. Journal of materials science. Materials in medicine. Jul 2016;27(7):118.

21. Kersten RF, van Gaalen SM, de Gast A, Oner FC. Polyetheretherketone (PEEK) cages in cervical applications: a systematic review. The spine journal : official journal of the North American Spine Society. Dec 262013.

22. Attwood TE, Dawson PC, Freeman JL, Hoy LRJ, Rose JB, Staniland PA. Synthesis and properties of polyaryletherketones. Polymer. 1981/08/01 1981;22(8):1096-1103.

23. Ha SW, Kirch M, Birchler F, et al. Surface activation of polyetheretherketone (PEEK) and formation of calcium phosphate coatings by precipitation. Journal of materials science. Materials in medicine. Nov 1997;8(11):683-690.

24. Kurtz SM. PEEK Biomaterials Handbook. Elsevier Science; 2011.

25. Callister WD. Materials Science And Engineering: An Introduction. John Wiley \& Sons; 2007.

26. Cheng SZD, Cao MY, Wunderlich B. Glass transition and melting behavior of poly(oxy-1,4-phenyleneoxy-1,4phenylenecarbonyl-1,4-phenylene) (PEEK). Macromolecules. 1986/07/01 1986;19(7):1868-1876.

27. Skinner HB. Composite technology for total hip arthroplasty. Clinical orthopaedics and related research. Oct 1988(235):224236.

28. Brown SA, Hastings RS, Mason JJ, Moet A. Characterization of short-fibre reinforced thermoplastics for fracture fixation 
devices. Biomaterials. Oct 1990;11(8):541-547.

29. Cowie JMG, Arrighi V. Polymers : chemistry and physics of modern materials /J.M.G. Cowie, Valeria Arrighi. Boca Raton, FL: CRC Press; 2008.

30. Blundell DJ, Osborn BN. The morphology of poly(aryl-etherether-ketone). Polymer. 1983/08/01 1983;24(8):953-958.

31. Talbott MF, Springer GS, Berglund LA. The Effects of Crystallinity on the Mechanical Properties of PEEK Polymer and Graphite Fiber Reinforced PEEK. Journal of Composite Materials. 1987;21(11):1056-1081.

32. Kurtz SM, Plastics Design Library. PEEK biomaterials handbook. 1st ed. Oxford UK ; Waltham, MA: William Andrew; 2012.

33. Day M, Deslandes Y, Roovers J, Suprunchuk T. Effect of molecular weight on the crystallization behaviour of poly(aryl ether ether ketone): a differential scanning calorimetry study. Polymer. 1991/01/01 1991;32(7):1258-1266.

34. Williams DF, McNamara A, Turner RM. Potential of polyetheretherketone (PEEK) and carbon-fibre-reinforced PEEK in medical applications. Journal of Materials Science Letters. 1987;6(2):188-190.

35. Ruben RB, Fernandes PR, Folgado J. On the optimal shape of hip implants. Journal of biomechanics. Jan 10 2012;45(2):239246.

36. Gupta S, New AM, Taylor M. Bone remodelling inside a cemented resurfaced femoral head. Clinical biomechanics. Jul 2006;21(6):594-602.

37. Ong KL, Kurtz SM, Manley MT, Rushton N, Mohammed NA, Field RE. Biomechanics of the Birmingham hip resurfacing arthroplasty. The Journal of bone and joint surgery. British volume. Aug 2006;88(8):1110-1115.

38. Yildiz H, Ha SK, Chang FK. Composite hip prosthesis design. I. Analysis. Journal of biomedical materials research. Jan 1998;39(1):92-101.

39. Yildiz H, Chang FK, Goodman S. Composite hip prosthesis design. II. Simulation. Journal of biomedical materials research. Jan 1998;39(1):102-119.

40. Wolff J. Concept of the Law of Bone Remodelling. The Law of Bone Remodelling. Berlin, Heidelberg: Springer Berlin Heidelberg; 1986:1-1.

41. Li CS, Vannabouathong C, Sprague S, Bhandari M. The Use of Carbon-Fiber-Reinforced (CFR) PEEK Material in Orthopedic Implants: A Systematic Review. Clinical medicine insights. Arthritis and musculoskeletal disorders. 2015;8:33-45. 
42. Sarot JR, Contar CM, Cruz AC, de Souza Magini R. Evaluation of the stress distribution in CFR-PEEK dental implants by the three-dimensional finite element method. Journal of materials science. Materials in medicine. Jul 2010;21(7):2079-2085.

43. Zhang G, Latour RA, Jr., Kennedy JM, Del Schutte H, Jr., Friedman RJ. Long-term compressive property durability of carbon fibre-reinforced polyetheretherketone composite in physiological saline. Biomaterials. Apr 1996;17(8):781-789.

44. Hamdan S, Swallowe GM. The strain-rate and temperature dependence of the mechanical properties of polyetherketone and polyetheretherketone. Journal of Materials Science. 1996;31(6):1415-1423.

45. Boinard E, Pethrick RA, MacFarlane CJ. The influence of thermal history on the dynamic mechanical and dielectric studies of polyetheretherketone exposed to water and brine. Polymer. 2// 2000;41(3):1063-1076.

46. Rae PJ, Brown EN, Orler EB. The mechanical properties of poly(ether-ether-ketone) (PEEK) with emphasis on the large compressive strain response. Polymer. 1/12/ 2007;48(2):598615.

47. Chivers RA, Moore DR. The effect of molecular weight and crystallinity on the mechanical properties of injection moulded poly(aryl-ether-ether-ketone) resin. Polymer. 1994/01/01 1994;35(1):110-116.

48. Victrex. Materials Properties Guide. In: Victrex, ed. West Conshohocken, PA2010.

49. Kemmish DJ, Hay JN. The effect of physical ageing on the properties of amorphous PEEK. Polymer. 1985/06/01 1985;26(6):905-912.

50. Roberts VI, Esler CN, Harper WM. A 15-year follow-up study of 4606 primary total knee replacements. The Journal of bone and joint surgery. British volume. Nov 2007;89(11):14521456.

51. Scholes SC, Unsworth A. Wear studies on the likely performance of CFR-PEEK/CoCrMo for use as artificial joint bearing materials. Journal of materials science. Materials in medicine. Jan 2009;20(1):163-170.

52. Scholes SC, Unsworth A. The wear properties of CFR-PEEKOPTIMA articulating against ceramic assessed on a multidirectional pin-on-plate machine. Proceedings of the Institution of Mechanical Engineers. Part H, Journal of engineering in medicine. Apr 2007;221(3):281-289.

53. Howling GI, Sakoda H, Antonarulrajah A, et al. Biological response to wear debris generated in carbon based composites 
as potential bearing surfaces for artificial hip joints. Journal of biomedical materials research. Part B, Applied biomaterials. Nov 15 2003;67(2):758-764.

54. Pace N MM, Spurio S, Di Matteo R. . Primary total hip arthroplasty with carbon fibre reinforced poly-ether-etherketone composite acetabular cup component 36 months results. Transactions of the International Society for Technology in Arthroplasty (ISTA). 2005;18:2-6.

55. Stober EJ, Seferis JC, Keenan JD. Characterization and exposure of polyetheretherketone (PEEK) to fluid environments. Polymer. 1984/12/01 1984;25(12):1845-1852.

56. Liebermann A, Wimmer T, Schmidlin PR, et al. Physicomechanical characterization of polyetheretherketone and current esthetic dental CAD/CAM polymers after aging in different storage media. The Journal of prosthetic dentistry. 3// 2016;115(3):321-328.e322.

57. Hay JN, Kemmish DJ. Thermal decomposition of poly(aryl ether ketones). Polymer. 1987/11/01 1987;28(12):2047-2051.

58. Buggy M, Carew A. The effect of thermal ageing on carbon fibre-reinforced polyetheretherketone (PEEK). Journal of Materials Science. 1994;29(8):2255-2259.

59. Kwarteng KB, Stark C. Carbon fiber reinforced PEEK (APC2/AS-4) composites for orthopaedic implants. Sampe Quarterly. 1990;22(1):10-14.

60. Godara A, Raabe D, Green S. The influence of sterilization processes on the micromechanical properties of carbon fiberreinforced PEEK composites for bone implant applications. Acta biomaterialia. Mar 2007;3(2):209-220.

61. Jahan MS, Wang C, Schwartz G, Davidson JA. Combined chemical and mechanical effects on free radicals in UHMWPE joints during implantation. Journal of biomedical materials research. Aug 1991;25(8):1005-1017.

62. Sasuga T, Hagiwara M. Radiation deterioration of several aromatic polymers under oxidative conditions. Polymer. 1987/10/01 1987;28(11):1915-1921.

63. Wenz LM, Merritt K, Brown SA, Moet A, Steffee AD. In vitro biocompatibility of polyetheretherketone and polysulfone composites. Journal of biomedical materials research. Feb 1990;24(2):207-215.

64. Lee WT, Koak JY, Lim YJ, Kim SK, Kwon HB, Kim MJ. Stress shielding and fatigue limits of poly-ether-ether-ketone dental implants. Journal of biomedical materials research. Part B, Applied biomaterials. May 2012;100(4):1044-1052.

65. Bezuidenhout D, Williams DF, Zilla P. Polymeric heart valves 
for surgical implantation, catheter-based technologies and heart assist devices. Biomaterials. Jan 2015;36:6-25.

66. Leat ME, Fisher J. A synthetic leaflet heart valve with improved opening characteristics. Medical engineering \& physics. Nov 1994;16(6):470-476.

67. Liu XY, Wang YP, Qiu GX, Weng XS, Yu B. Meta-analysis of circumferential fusion versus posterolateral fusion in lumbar spondylolisthesis. Journal of spinal disorders \& techniques. Dec 2014;27(8):E282-293.

68. Liu X, Wang Y, Qiu G, Weng X, Yu B. A systematic review with meta-analysis of posterior interbody fusion versus posterolateral fusion in lumbar spondylolisthesis. European spine journal : official publication of the European Spine Society, the European Spinal Deformity Society, and the European Section of the Cervical Spine Research Society. Jan 2014;23(1):43-56.

69. Bevevino AJ, Kang DG, Lehman RA, Jr., Van Blarcum GS, Wagner SC, Gwinn DE. Systematic review and meta-analysis of minimally invasive transforaminal lumbar interbody fusion rates performed without posterolateral fusion. Journal of clinical neuroscience : official journal of the Neurosurgical Society of Australasia. Oct 2014;21(10):1686-1690.

70. Brantigan JW, Lauryssen C. Intervertebral Fusion Using Carbon Fiber Reinforced Polymer Implants. St. Louis: Quality Medical Publishing; 2006.

71. Ponnappan RK, Serhan H, Zarda B, Patel R, Albert T, Vaccaro AR. Biomechanical evaluation and comparison of polyetheretherketone rod system to traditional titanium rod fixation. The spine journal : official journal of the North American Spine Society. Mar 2009;9(3):263-267.

72. Ha SK, Park JY, Kim SH, Lim DJ, Kim SD, Lee SK. Radiologic Assessment of Subsidence in Stand-Alone Cervical Polyetheretherketone (PEEK) Cage. Journal of Korean Neurosurgical Society. Dec 2008;44(6):370-374.

73. Cho DY, Liau WR, Lee WY, Liu JT, Chiu CL, Sheu PC. Preliminary experience using a polyetheretherketone (PEEK) cage in the treatment of cervical disc disease. Neurosurgery. Dec 2002;51(6):1343-1349; discussion 1349-1350.

74. Christensen FB, Hansen ES, Eiskjaer SP, et al. Circumferential lumbar spinal fusion with Brantigan cage versus posterolateral fusion with titanium Cotrel-Dubousset instrumentation: a prospective, randomized clinical study of 146 patients. Spine. Dec 01 2002;27(23):2674-2683.

75. Videbaek TS, Christensen FB, Soegaard R, et al. 
Circumferential fusion improves outcome in comparison with instrumented posterolateral fusion: long-term results of a randomized clinical trial. Spine. Dec 01 2006;31(25):28752880 .

76. Brantigan JW, Steffee AD. A carbon fiber implant to aid interbody lumbar fusion. Two-year clinical results in the first 26 patients. Spine. Oct 15 1993;18(14):2106-2107.

77. Brantigan JW, McAfee PC, Cunningham BW, Wang H, Orbegoso CM. Interbody lumbar fusion using a carbon fiber cage implant versus allograft bone. An investigational study in the Spanish goat. Spine. Jul 01 1994;19(13):1436-1444.

78. Ni J, Zheng Y, Liu N, et al. Radiological evaluation of anterior lumbar fusion using PEEK cages with adjacent vertebral autograft in spinal deformity long fusion surgeries. European spine journal : official publication of the European Spine Society, the European Spinal Deformity Society, and the European Section of the Cervical Spine Research Society. Apr 2015;24(4):791-799.

79. Kao TH, Wu CH, Chou YC, Chen HT, Chen WH, Tsou HK. Risk factors for subsidence in anterior cervical fusion with stand-alone polyetheretherketone (PEEK) cages: a review of 82 cases and 182 levels. Archives of orthopaedic and trauma surgery. Oct 2014;134(10):1343-1351.

80. Behrbalk E, Uri O, Parks RM, Musson R, Soh RC, Boszczyk $\mathrm{BM}$. Fusion and subsidence rate of stand alone anterior lumbar interbody fusion using PEEK cage with recombinant human bone morphogenetic protein-2. European spine journal : official publication of the European Spine Society, the European Spinal Deformity Society, and the European Section of the Cervical Spine Research Society. Dec 2013;22(12):28692875.

81. Bartels RH, Donk RD, Feuth T. Subsidence of stand-alone cervical carbon fiber cages. Neurosurgery. Mar 2006;58(3):502-508; discussion 502-508.

82. Tullberg T. Failure of a carbon fiber implant. A case report. Spine. Aug 15 1998;23(16):1804-1806.

83. Wang A, Lin R, Stark C, Dumbleton JH. Suitability and limitations of carbon fiber reinforced PEEK composites as bearing surfaces for total joint replacements. Wear. 4// 1999;225-229, Part 2:724-727.

84. Sumner DR. Long-term implant fixation and stress-shielding in total hip replacement. Journal of biomechanics. Mar 18 2015;48(5):797-800.

85. Bauer TW, Schils J. The pathology of total joint arthroplasty.II. 
Mechanisms of implant failure. Skeletal radiology. Sep 1999;28(9):483-497.

86. Rao AJ, Gibon E, Ma T, Yao Z, Smith RL, Goodman SB. Revision joint replacement, wear particles, and macrophage polarization. Acta biomaterialia. Jul 2012;8(7):2815-2823.

87. Goodman SB. Wear particles, periprosthetic osteolysis and the immune system. Biomaterials. Dec 2007;28(34):5044-5048.

88. Green TR, Fisher J, Stone M, Wroblewski BM, Ingham E. Polyethylene particles of a 'critical size' are necessary for the induction of cytokines by macrophages in vitro. Biomaterials. Dec 1998;19(24):2297-2302.

89. Matthews JB, Besong AA, Green TR, et al. Evaluation of the response of primary human peripheral blood mononuclear phagocytes to challenge with in vitro generated clinically relevant UHMWPE particles of known size and dose. Journal of biomedical materials research. Nov 2000;52(2):296-307.

90. Stratton-Powell AA, Pasko KM, Brockett CL, Tipper JL. The Biologic Response to Polyetheretherketone (PEEK) Wear Particles in Total Joint Replacement: A Systematic Review. Clinical Orthopaedics and Related Research(B). 2016;474(11):2394-2404.

91. Li YD, Liu SM, Jia JS, Zhou JL. [Choice of internal fixation methods for posterior malleolus fracture in both biomechanics and clinical application]. Beijing da xue xue bao. Yi xue ban = Journal of Peking University. Health sciences. Oct 18 2011;43(5):718-723.

92. Tayton K, Bradley J. How stiff should semi-rigid fixation of the human tibia be? A clue to the answer. The Journal of bone and joint surgery. British volume. May 1983;65(3):312-315.

93. Fujihara K, Huang ZM, Ramakrishna S, Satknanantham K, Hamada H. Performance study of braided carbon/PEEK composite compression bone plates. Biomaterials. Jul 2003;24(15):2661-2667.

94. Schliemann B, Hartensuer R, Koch $\mathrm{T}$, et al. Treatment of proximal humerus fractures with a CFR-PEEK plate: 2-year results of a prospective study and comparison to fixation with a conventional locking plate. Journal of shoulder and elbow surgery / American Shoulder and Elbow Surgeons ... [et al.]. Aug 2015;24(8):1282-1288.

95. Schliemann B, Seifert R, Theisen C, et al. PEEK versus titanium locking plates for proximal humerus fracture fixation: a comparative biomechanical study in two- and three-part fractures. Archives of orthopaedic and trauma surgery. 2017;137(1):63-71. 
96. Uhthoff HK, Poitras P, Backman DS. Internal plate fixation of fractures: short history and recent developments. Journal of orthopaedic science : official journal of the Japanese Orthopaedic Association. Mar 2006;11(2):118-126.

97. O'Reilly EB, Barnett S, Madden C, Welch B, Mickey B, Rozen $\mathrm{S}$. Computed-tomography modeled polyether ether ketone (PEEK) implants in revision cranioplasty. Journal of plastic, reconstructive \& aesthetic surgery : JPRAS. Mar 2015;68(3):329-338.

98. Kuboki T, Okamoto S, Suzuki H, et al. Quality of life assessment of bone-anchored fixed partial denture patients with unilateral mandibular distal-extension edentulism. The Journal of prosthetic dentistry. Aug 1999;82(2):182-187.

99. Steinemann SG. Titanium--the material of choice? Periodontology 2000. Jun 1998;17:7-21.

100. Misumi S, Nakamoto T, Kondo Y, Mukaibo T, Masaki C, Hosokawa R. A prospective study of changes in oral healthrelated quality of life during immediate function implant procedures for edentulous individuals. Clinical oral implants research. Jun 2015;26(6):696-700.

101. Chappuis V, Buser R, Bragger U, Bornstein MM, Salvi GE, Buser D. Long-term outcomes of dental implants with a titanium plasma-sprayed surface: a 20 -year prospective case series study in partially edentulous patients. Clinical implant dentistry and related research. Dec 2013;15(6):780-790.

102. Holgers KM, Roupe G, Tjellstrom A, Bjursten LM. Clinical, immunological and bacteriological evaluation of adverse reactions to skin-penetrating titanium implants in the head and neck region. Contact dermatitis. Jul 1992;27(1):1-7.

103. Goutam M, Giriyapura C, Mishra SK, Gupta S. Titanium allergy: a literature review. Indian journal of dermatology. Nov 2014;59(6):630.

104. Thomas P, Iglhaut G, Wollenberg A, Cadosch D, Summer B. Allergy or tolerance: reduced inflammatory cytokine response and concomitant IL-10 production of lymphocytes and monocytes in symptom-free titanium dental implant patients. BioMed research international. 2013;2013:539834.

105. Prochazkova J, Podzimek S, Tomka M, et al. Metal alloys in the oral cavity as a cause of oral discomfort in sensitive patients. Neuro endocrinology letters. Dec 2006;27 Suppl 1:5358.

106. Rezaei F, Hassani K, Solhjoei N, Karimi A. Carbon/PEEK composite materials as an alternative for stainless steel/titanium hip prosthesis: a finite element study. Australasian physical \& 
engineering sciences in medicine / supported by the Australasian College of Physical Scientists in Medicine and the Australasian Association of Physical Sciences in Medicine. Dec 2015;38(4):569-580.

107. Andreiotelli M, Wenz HJ, Kohal RJ. Are ceramic implants a viable alternative to titanium implants? A systematic literature review. Clinical oral implants research. Sep 2009;20 Suppl 4:32-47.

108. Aydin C, Yilmaz H, Ata SO. Single-tooth zirconia implant located in anterior maxilla. A clinical report. The New York state dental journal. Jan 2010;76(1):30-33.

109. Ozkurt Z, Kazazoglu E. Clinical success of zirconia in dental applications. Journal of prosthodontics : official journal of the American College of Prosthodontists. Jan 2010;19(1):64-68.

110. Moon SM, Ingalhalikar A, Highsmith JM, Vaccaro AR. Biomechanical rigidity of an all-polyetheretherketone anterior thoracolumbar spinal reconstruction construct: an in vitro corpectomy model. The spine journal : official journal of the North American Spine Society. Apr 2009;9(4):330-335.

111. Huang RYM, Shao P, Burns CM, Feng X. Sulfonation of poly(ether ether ketone)(PEEK): Kinetic study and characterization. Journal of Applied Polymer Science. 2001;82(11):2651-2660.

112. Nieminen T, Kallela I, Wuolijoki E, Kainulainen $H$, Hiidenheimo I, Rantala I. Amorphous and crystalline polyetheretherketone: Mechanical properties and tissue reactions during a 3-year follow-up. Journal of biomedical materials research. Part A. Feb 2008;84(2):377-383.

113. Koch FP, Weng D, Kramer S, Biesterfeld S, Jahn-Eimermacher A, Wagner W. Osseointegration of one-piece zirconia implants compared with a titanium implant of identical design: a histomorphometric study in the dog. Clinical oral implants research. Mar 2010;21(3):350-356.

114. Schwitalla AD, Muller WD. PEEK dental implants: A Review of the Literature. The Journal of oral implantology. Sep 92011.

115. Theofilos Koutouzis, Joseph Richardson, Tord Lundgren. Comparative Soft and Hard Tissue Responses to Titanium and Polymer Healing Abutments. Journal of Oral Implantology. 2011;37(sp1):174-182.

116. Hahnel S, Wieser A, Lang R, Rosentritt M. Biofilm formation on the surface of modern implant abutment materials. Clinical oral implants research. Nov 2015;26(11):1297-1301.

117. Santing HJ, Meijer HJ, Raghoebar GM, Ozcan M. Fracture strength and failure mode of maxillary implant-supported 
provisional single crowns: a comparison of composite resin crowns fabricated directly over PEEK abutments and solid titanium abutments. Clinical implant dentistry and related research. Dec 2012;14(6):882-889.

118. Agustin-Panadero R, Serra-Pastor B, Roig-Vanaclocha A, Roman-Rodriguez JL, Fons-Font A. Mechanical behavior of provisional implant prosthetic abutments. Medicina oral, patologia oral y cirugia bucal. Jan 01 2015;20(1):e94-102.

119. Tannous F, Steiner M, Shahin R, Kern M. Retentive forces and fatigue resistance of thermoplastic resin clasps. Dental materials : official publication of the Academy of Dental Materials. Mar 2012;28(3):273-278.

120. Costa-Palau S, Torrents-Nicolas J, Brufau-de Barbera M, Cabratosa-Termes J. Use of polyetheretherketone in the fabrication of a maxillary obturator prosthesis: a clinical report. The Journal of prosthetic dentistry. Sep 2014;112(3):680-682.

121. Sproesser O, Schmidlin PR, Uhrenbacher J, Roos M, Gernet W, Stawarczyk B. Effect of sulfuric acid etching of polyetheretherketone on the shear bond strength to resin cements. The journal of adhesive dentistry. Oct 2014;16(5):465-472.

122. Stawarczyk B, Eichberger M, Uhrenbacher J, Wimmer T, Edelhoff D, Schmidlin PR. Three-unit reinforced polyetheretherketone composite FDPs: influence of fabrication method on load-bearing capacity and failure types. Dental materials journal. 2015;34(1):7-12.

123. Wright TW. Definitions in Biomaterials. Journal of biomechanics.22(1):79.

124. Greenwald AS. Biological Performance of Materials. Fundamentals of Biocompatibility. 3rd ed. JBJS. 2001;83(6):970.

125. Petillo O, Peluso G, Ambrosio L, Nicolais L, Kao WJ, Anderson JM. In vivo induction of macrophage Ia antigen (MHC class II) expression by biomedical polymers in the cage implant system. Journal of biomedical materials research. May 1994;28(5):635646.

126. Morrison C, Macnair R, MacDonald C, Wykman A, Goldie I, Grant $\mathrm{MH}$. In vitro biocompatibility testing of polymers for orthopaedic implants using cultured fibroblasts and osteoblasts. Biomaterials. Sep 1995;16(13):987-992.

127. Katzer A, Marquardt H, Westendorf J, Wening JV, von Foerster G. Polyetheretherketone--cytotoxicity and mutagenicity in vitro. Biomaterials. Apr 2002;23(8):1749- 
1759.

128. Macnair R, Rodgers EH, Macdonald C, Wykman A, Goldie I, Grant $\mathrm{MH}$. The response of primary rat and human osteoblasts and an immortalized rat osteoblast cell line to orthopaedic materials: comparative sensitivity of several toxicity indices. Journal of materials science. Materials in medicine. Feb 1997;8(2):105-111.

129. Hunter A, Archer CW, Walker PS, Blunn GW. Attachment and proliferation of osteoblasts and fibroblasts on biomaterials for orthopaedic use. Biomaterials. Mar 1995;16(4):287-295.

130. Jiang Y, Jia T, Wooley PH, Yang SY. Current research in the pathogenesis of aseptic implant loosening associated with particulate wear debris. Acta orthopaedica Belgica. Feb 2013;79(1):1-9.

131. Rivard $\mathrm{CH}$, Rhalmi S, Coillard C. In vivo biocompatibility testing of peek polymer for a spinal implant system: a study in rabbits. Journal of biomedical materials research. Dec 15 2002;62(4):488-498.

132. Jockisch KA, Brown SA, Bauer TW, Merritt K. Biological response to chopped-carbon-fiber-reinforced peek. Journal of biomedical materials research. Feb 1992;26(2):133-146.

133. Hallab NJ, McAllister K, Brady M, Jarman-Smith $M$. Macrophage reactivity to different polymers demonstrates particle size- and material-specific reactivity: PEEKOPTIMA $((\mathrm{R}))$ particles versus UHMWPE particles in the submicron, micron, and 10 micron size ranges. Journal of biomedical materials research. Part B, Applied biomaterials. Nov 212011.

134. Toth JM, Wang M, Estes BT, Scifert JL, Seim HB, 3rd, Turner AS. Polyetheretherketone as a biomaterial for spinal applications. Biomaterials. Jan 2006;27(3):324-334.

135. Wang $\mathrm{H}, \mathrm{Xu} \mathrm{M}$, Zhang W, et al. Mechanical and biological characteristics of diamond-like carbon coated poly aryl-etherether-ketone. Biomaterials. Nov 2010;31(32):8181-8187.

136. Roeder RK, Converse GL, Kane RJ, Yue W. Hydroxyapatitereinforced polymer biocomposites for synthetic bone substitutes. JOM. 2008;60(3):38-45.

137. Rezwan K, Chen QZ, Blaker JJ, Boccaccini AR. Biodegradable and bioactive porous polymer/inorganic composite scaffolds for bone tissue engineering. Biomaterials. 6// 2006;27(18):3413-3431.

138. Abu Bakar MS, Cheng MH, Tang SM, et al. Tensile properties, tension-tension fatigue and biological response of polyetheretherketone-hydroxyapatite composites for load- 
bearing orthopedic implants. Biomaterials. Jun 2003;24(13):2245-2250.

139. Wong KL, Wong CT, Liu WC, et al. Mechanical properties and in vitro response of strontium-containing hydroxyapatite/polyetheretherketone composites. Biomaterials. Aug 2009;30(23-24):3810-3817.

140. Kokubo T, Kim HM, Kawashita M. Novel bioactive materials with different mechanical properties. Biomaterials. Jun 2003;24(13):2161-2175.

141. Okuda T, Ioku K, Yonezawa I, et al. The slow resorption with replacement by bone of a hydrothermally synthesized pure calcium-deficient hydroxyapatite. Biomaterials. Jun 2008;29(18):2719-2728.

142. Hench LL. Bioceramics: From Concept to Clinic. American Ceramic Society; 1991.

143. LeGeros RZ. Properties of osteoconductive biomaterials: calcium phosphates. Clinical orthopaedics and related research. Feb 2002(395):81-98.

144. Abu Bakar MS, Cheang P, Khor KA. Thermal processing of hydroxyapatite reinforced polyetheretherketone composites. Journal of Materials Processing Technology. 5/19/ 1999;8990:462-466.

145. Abu Bakar MS, Cheang P, Khor KA. Mechanical properties of injection molded hydroxyapatite-polyetheretherketone biocomposites. Composites Science and Technology. 2// 2003;63(3-4):421-425.

146. Tang SM, Cheang P, AbuBakar MS, Khor KA, Liao K. Tension-tension fatigue behavior of hydroxyapatite reinforced polyetheretherketone composites. International Journal of Fatigue. 1// 2004;26(1):49-57.

147. Tan KH, Chua CK, Leong KF, et al. Scaffold development using selective laser sintering of polyetheretherketonehydroxyapatite biocomposite blends. Biomaterials. 8// 2003;24(18):3115-3123.

148. Tan KH, Chua CK, Leong KF, Naing MW, Cheah CM. Fabrication and characterization of three-dimensional poly(ether- ether- ketone)/-hydroxyapatite biocomposite scaffolds using laser sintering. Proceedings of the Institution of Mechanical Engineers. Part H, Journal of engineering in medicine. May 2005;219(3):183-194.

149. Yu S, Hariram KP, Kumar R, Cheang P, Aik KK. In vitro apatite formation and its growth kinetics on hydroxyapatite/polyetheretherketone biocomposites. Biomaterials. May 2005;26(15):2343-2352. 
150. Hengky C, Kelsen B, Saraswati, Cheang P. Mechanical and Biological Characterization of Pressureless Sintered Hydroxapatite-Polyetheretherketone Biocomposite. In: Lim CT, Goh JCH, eds. 13th International Conference on Biomedical Engineering: ICBME 2008 3-6 December 2008 Singapore. Berlin, Heidelberg: Springer Berlin Heidelberg; 2009:261-264.

151. Petrovic L, Pohle D, Munstedt H, Rechtenwald T, Schlegel KA, Rupprecht S. Effect of betaTCP filled polyetheretherketone on osteoblast cell proliferation in vitro. Journal of biomedical science. Jan 2006;13(1):41-46.

152. Pohle D, Ponader S, Rechtenwald T, et al. Processing of ThreeDimensional Laser Sintered Polyetheretherketone Composites and Testing of Osteoblast Proliferation in vitro. Macromolecular Symposia. 2007;253(1):65-70.

153. von Wilmowsky C, Vairaktaris E, Pohle D, et al. Effects of bioactive glass and beta-TCP containing three-dimensional laser sintered polyetheretherketone composites on osteoblasts in vitro. Journal of biomedical materials research. Part A. Dec 15 2008;87(4):896-902.

154. Wilmonsky CV, Lutz R, Meisel U, et al. In Vivo Evaluation of B-TCP Containing 3D Laser Sintered Poly(ether ether ketone) Composites in Pigs. Journal of Bioactive and Compatible Polymers. 2009;24(2):169-184.

155. Converse GL, Yue W, Roeder RK. Processing and tensile properties of hydroxyapatite-whisker-reinforced polyetheretherketone. Biomaterials. Feb 2007;28(6):927-935.

156. Kim IY, Sugino A, Kikuta K, Ohtsuki C, Cho SB. Bioactive composites consisting of PEEK and calcium silicate powders. Journal of biomaterials applications. Aug 2009;24(2):105-118.

157. Meenan BJ, McClorey C, Akay M. Thermal analysis studies of poly(etheretherketone)/hydroxyapatite biocomposite mixtures. Journal of materials science. Materials in medicine. Aug 2000;11(8):481-489.

158. Boccaccini AR, Peters C, Roether JA, Eifler D, Misra SK, Minay EJ. Electrophoretic deposition of polyetheretherketone (PEEK) and PEEK/Bioglass ${ }^{\circledR}$ coatings on NiTi shape memory alloy wires. Journal of Materials Science. 2006;41(24):81528159.

159. Abu Bakar MS, Cheang P, Khor KA. Tensile properties and microstructural analysis of spheroidized hydroxyapatite-poly (etheretherketone) biocomposites. Materials Science and Engineering: A. 3/25/ 2003;345(1-2):55-63.

160. Zhang Y, Hao L, Savalani MM, Harris RA, Di Silvio L, Tanner 
KE. In vitro biocompatibility of hydroxyapatite-reinforced polymeric composites manufactured by selective laser sintering. Journal of biomedical materials research. Part A. Dec 15 2009;91(4):1018-1027.

161. Shu R, McMullen R, Baumann MJ, McCabe LR. Hydroxyapatite accelerates differentiation and suppresses growth of MC3T3-E1 osteoblasts. Journal of biomedical materials research. Part A. Dec 15 2003;67(4):1196-1204.

162. Kokubo T, Takadama H. How useful is SBF in predicting in vivo bone bioactivity? Biomaterials. May 2006;27(15):29072915.

163. Pors Nielsen S. The biological role of strontium. Bone. Sep 2004;35(3):583-588.

164. Lin TW, Corvelli AA, Frondoza CG, Roberts JC, Hungerford DS. Glass peek composite promotes proliferation and osteocalcin production of human osteoblastic cells. Journal of biomedical materials research. Aug 1997;36(2):137-144.

165. Corvelli AA, Roberts JC, Biermann PJ, Cranmer JH. Characterization of a peek composite segmental bone replacement implant. Journal of Materials Science. 1999;34(10):2421-2431.

166. Ofluoglu AE, Erdogan U, Aydogan M, Cevik OM, Ofluoglu O. Anterior cervical fusion with interbody cage containing betatricalcium phosphate: Clinical and radiological results. Acta orthopaedica et traumatologica turcica. Mar 252017.

167. Wang L, Weng L, Song S, Sun Q. Mechanical properties and microstructure of polyetheretherketone-hydroxyapatite nanocomposite materials. Materials Letters. 2010/10/31/ 2010;64(20):2201-2204.

168. Wang L, Weng L, Song S, Zhang Z, Tian S, Ma R. Characterization of polyetheretherketone-hydroxyapatite nanocomposite materials. Materials Science and Engineering: A. 4/25/ 2011;528(10-11):3689-3696.

169. Ma R, Weng L, Fang L, Luo Z, Song S. Structure and mechanical performance of in situ synthesized hydroxyapatite/polyetheretherketone nanocomposite materials. Journal of Sol-Gel Science and Technology. 2012;62(1):52-56.

170. Kriparamanan R, Aswath P, Zhou A, Tang L, Nguyen KT. Nanotopography: cellular responses to nanostructured materials. Journal of nanoscience and nanotechnology. Jul 2006;6(7):1905-1919.

171. Stevens B, Yang Y, Mohandas A, Stucker B, Nguyen KT. A review of materials, fabrication methods, and strategies used to 
enhance bone regeneration in engineered bone tissues. Journal of biomedical materials research. Part B, Applied biomaterials. May 2008;85(2):573-582.

172. Njuguna J, Pielichowski K, Desai S. Nanofiller-reinforced polymer nanocomposites. Polymers for Advanced Technologies. 2008;19(8):947-959.

173. Díez-Pascual AM, Naffakh M, Marco C, Ellis G, Gómez-Fatou MA. High-performance nanocomposites based on polyetherketones. Progress in Materials Science. 9// 2012;57(7):1106-1190.

174. Webster TJ, Siegel RW, Bizios R. Osteoblast adhesion on nanophase ceramics. Biomaterials. Jul 1999;20(13):12211227.

175. Webster TJ, Ergun C, Doremus RH, Siegel RW, Bizios R. Enhanced functions of osteoblasts on nanophase ceramics. Biomaterials. Sep 2000;21(17):1803-1810.

176. Gutwein LG, Webster TJ. Osteoblast and Chrondrocyte Proliferation in the Presence of Alumina And Titania Nanoparticles. Journal of Nanoparticle Research. 2002;4(3):231-238.

177. Li K, Yeung CY, Yeung KWK, Tjong SC. Sintered Hydroxyapatite/Polyetheretherketone Nanocomposites: Mechanical Behavior and Biocompatibility. Advanced Engineering Materials. 2012;14(4):B155-B165.

178. Wu X, Liu X, Wei J, Ma J, Deng F, Wei S. Nano-TiO2/PEEK bioactive composite as a bone substitute material: in vitro and in vivo studies. International journal of nanomedicine. 2012;7:1215-1225.

179. Zhao M, Li H, Liu X, et al. Response of Human Osteoblast to n-HA/PEEK--Quantitative Proteomic Study of Bio-effects of Nano-Hydroxyapatite Composite. Scientific reports. Mar 09 2016;6:22832.

180. Deng QX, Ou YS, Zhu Y, et al. Clinical outcomes of two types of cages used in transforaminal lumbar interbody fusion for the treatment of degenerative lumbar diseases: n-HA/PA66 cages versus PEEK cages. Journal of materials science. Materials in medicine. Jun 2016;27(6):102.

181. Wang L, He S, Wu X, et al. Polyetheretherketone/nanofluorohydroxyapatite composite with antimicrobial activity and osseointegration properties. Biomaterials. Aug 2014;35(25):6758-6775.

182. Hegemann D, Brunner $\mathrm{H}$, Oehr C. Plasma treatment of polymers for surface and adhesion improvement. Nuclear Instruments and Methods in Physics Research Section B: Beam 
Interactions with Materials and Atoms. 2003/08/01/ 2003;208:281-286.

183. Edward L. Plasma Treatment for Improved Bonding: A Review. The Journal of Adhesion. 1989/01/01 1989;30(1-4):199-218.

184. Briem D, Strametz S, Schroder K, et al. Response of primary fibroblasts and osteoblasts to plasma treated polyetheretherketone (PEEK) surfaces. Journal of materials science. Materials in medicine. Jul 2005;16(7):671-677.

185. Awaja F, Bax DV, Zhang S, James N, McKenzie DR. Cell Adhesion to PEEK Treated by Plasma Immersion Ion Implantation and Deposition for Active Medical Implants. Plasma Processes and Polymers. 2012;9(4):355-362.

186. Awaja F, Zhang S, James N, McKenzie DR. Enhanced Autohesive Bonding of Polyetheretherketone (PEEK) for Biomedical Applications Using a Methane/Oxygen Plasma Treatment. Plasma Processes and Polymers. 2010;7(12):10101021.

187. Khoury J KS, Maxwell M, Cherian RE, Kirkpatrick A, Svrluga RC. Neutral atom beam technique enhances bioactivity of PEEK. Nucl. Indstr. Meth B. 2013(307):630-634.

188. Zhao Y, Wong HM, Wang W, et al. Cytocompatibility, osseointegration, and bioactivity of three-dimensional porous and nanostructured network on polyetheretherketone. Biomaterials. Dec 2013;34(37):9264-9277.

189. Miyazaki T, Matsunami C, Shirosaki Y. Bioactive carbonPEEK composites prepared by chemical surface treatment. Materials science \& engineering. C, Materials for biological applications. Jan 01 2017;70(Pt 1):71-75.

190. Hieda A, Uemura N, Hashimoto Y, Toda I, Baba S. In vivo bioactivity of porous polyetheretherketone with a foamed surface. Dental materials journal. Mar 31 2017;36(2):222-229.

191. Ouyang L, Zhao Y, Jin G, et al. Influence of sulfur content on bone formation and antibacterial ability of sulfonated PEEK. Biomaterials. Mar 2016;83:115-126.

192. Hench LL. Bioceramics and the origin of life. Journal of biomedical materials research. Jul 1989;23(7):685-703.

193. Lee JH, Jang HL, Lee KM, et al. In vitro and in vivo evaluation of the bioactivity of hydroxyapatite-coated polyetheretherketone biocomposites created by cold spray technology. Acta biomaterialia. 4// 2013;9(4):6177-6187.

194. Hahn B-D, Park D-S, Choi J-J, et al. Osteoconductive hydroxyapatite coated PEEK for spinal fusion surgery. Applied Surface Science. 10/15/ 2013;283:6-11.

195. Rabiei A, Sandukas S. Processing and evaluation of bioactive 
coatings on polymeric implants. Journal of biomedical materials research. Part A. Feb 152013.

196. Jung H-D, Sun Park H, Kang M-H, et al. Polyetheretherketone/magnesium composite selectively coated with hydroxyapatite for enhanced in vitro bio-corrosion resistance and biocompatibility. Materials Letters. 2/1/ 2014;116:20-22.

197. Barkarmo S, Wennerberg A, Hoffman M, et al. Nanohydroxyapatite-coated PEEK implants: a pilot study in rabbit bone. Journal of biomedical materials research. Part A. Feb 2013;101(2):465-471.

198. Ozeki K, Masuzawa T, Aoki H. Fabrication of hydroxyapatite thin films on polyetheretherketone substrates using a sputtering technique. Materials Science and Engineering: C. 3/1/ 2017;72:576-582.

199. Van Noort R. Titanium: The implant material of today. Journal of Materials Science. 1987;22(11):3801-3811.

200. Yao C, Storey D, Webster TJ. Nanostructured metal coatings on polymers increase osteoblast attachment. International journal of nanomedicine. 09/ 2007;2(3):487-492.

201. Cook SD, Rust-Dawicki AM. Preliminary evaluation of titanium-coated PEEK dental implants. The Journal of oral implantology. 1995;21(3):176-181.

202. Han CM, Lee EJ, Kim HE, et al. The electron beam deposition of titanium on polyetheretherketone (PEEK) and the resulting enhanced biological properties. Biomaterials. May 2010;31(13):3465-3470.

203. Ha SW, Eckert KL, Wintermantel E, Gruner H, Guecheva M, Vonmont $\mathrm{H}$. $\mathrm{NaOH}$ treatment of vacuum-plasma-sprayed titanium on carbon fibre-reinforced poly(etheretherketone). Journal of materials science. Materials in medicine. Dec 1997;8(12):881-886.

204. Walsh WR, Bertollo N, Christou C, Schaffner D, Mobbs RJ. Plasma-sprayed titanium coating to polyetheretherketone improves the bone-implant interface. The spine journal : official journal of the North American Spine Society. May 01 2015;15(5):1041-1049.

205. Tsou HK, Hsieh PY, Chi MH, Chung CJ, He JL. Improved osteoblast compatibility of medical-grade polyetheretherketone using arc ionplated rutile/anatase titanium dioxide films for spinal implants. Journal of biomedical materials research. Part A. Oct 2012;100(10):2787-2792.

206. Chi M-H, Tsou H-K, Chung C-J, He J-L. Biomimetic hydroxyapatite grown on biomedical polymer coated with 
titanium dioxide interlayer to assist osteocompatible performance. Thin Solid Films. 2013/12/31/ 2013;549:98-102.

207. Han CM, Jang TS, Kim HE, Koh YH. Creation of nanoporous $\mathrm{TiO} 2$ surface onto polyetheretherketone for effective immobilization and delivery of bone morphogenetic protein. Journal of biomedical materials research. Part A. Mar 2014;102(3):793-800.

208. Shimizu T, Fujibayashi S, Yamaguchi S, et al. Bioactivity of solgel-derived $\mathrm{TiO} 2$ coating on polyetheretherketone: In vitro and in vivo studies. Acta biomaterialia. 4/15/ 2016;35:305-317.

209. Katsuda SI, Shimizu T, Yamasaki M, et al. Mechanical tensile properties of the aortic wall in the premature rat exposed to the microgravity environment during space flight for 16 days. Journal of gravitational physiology : a journal of the International Society for Gravitational Physiology. Jul 2000;7(2):P157-158.

210. Kjellin P, Andersson M. Synthetic nano-sized crystalline calcium phosphate and method of production. Google Patents; 2012.

211. Wennerberg A, Albrektsson T. Suggested guidelines for the topographic evaluation of implant surfaces. The International journal of oral \& maxillofacial implants. May-Jun 2000;15(3):331-344.

212. Morris VJ, Kirby AR, Gunning AP. Atomic Force Microscopy for Biologists. Imperial College Press; 1999.

213. Lim YJ, Oshida Y, Andres CJ, Barco MT. Surface characterizations of variously treated titanium materials. The International journal of oral \& maxillofacial implants. MayJun 2001;16(3):333-342.

214. Wang X, Mabrey JD, Agrawal CM. An interspecies comparison of bone fracture properties. Bio-medical materials and engineering. 1998;8(1):1-9.

215. Castaneda S, Largo R, Calvo E, et al. Bone mineral measurements of subchondral and trabecular bone in healthy and osteoporotic rabbits. Skeletal radiology. Jan 2006;35(1):34-41.

216. Roberts WE, Smith RK, Zilberman Y, Mozsary PG, Smith RS. Osseous adaptation to continuous loading of rigid endosseous implants. American journal of orthodontics. Aug 1984;86(2):95-111.

217. Roberts WE, Simmons KE, Garetto LP, DeCastro RA. Bone physiology and metabolism in dental implantology: risk factors for osteoporosis and other metabolic bone diseases. Implant dentistry. Spring 1992;1(1):11-21. 
218. Gilsanz V, Roe TF, Gibbens DT, et al. Effect of sex steroids on peak bone density of growing rabbits. The American journal of physiology. Oct 1988;255(4 Pt 1):E416-421.

219. Stenlund P, Murase K, Stalhandske C, Lausmaa J, Palmquist A. Understanding mechanisms and factors related to implant fixation; a model study of removal torque. Journal of the mechanical behavior of biomedical materials. Jun 2014;34:8392.

220. Donath K, Breuner G. A method for the study of undecalcified bones and teeth with attached soft tissues. The Sage-Schliff (sawing and grinding) technique. Journal of oral pathology. Aug 1982;11(4):318-326.

221. Albrektsson T, Wennerberg A. Oral implant surfaces: Part 1-review focusing on topographic and chemical properties of different surfaces and in vivo responses to them. The International journal of prosthodontics. Sep-Oct 2004;17(5):536-543.

222. Mekayarajjananonth T, Winkler S. Contact Angle Measurement on Dental Implant Biomaterials. Journal of Oral Implantology. 1999;25(4):230-236.

223. Albrektsson T, Branemark PI, Hansson HA, Lindstrom J. Osseointegrated titanium implants. Requirements for ensuring a long-lasting, direct bone-to-implant anchorage in man. Acta orthopaedica Scandinavica. 1981;52(2):155-170.

224. Berube P, Yang Y, Carnes DL, Stover RE, Boland EJ, Ong JL. The effect of sputtered calcium phosphate coatings of different crystallinity on osteoblast differentiation. Journal of periodontology. Oct 2005;76(10):1697-1709.

225. Hulshoff JE, Hayakawa T, van Dijk K, Leijdekkers-Govers AF, van der Waerden JP, Jansen JA. Mechanical and histologic evaluation of Ca-P plasma-spray and magnetron sputter-coated implants in trabecular bone of the goat. Journal of biomedical materials research. Jul 1997;36(1):75-83.

226. Carrado A, Perrin-Schmitt F, Le QV, et al. Nanoporous hydroxyapatite/sodium titanate bilayer on titanium implants for improved osteointegration. Dental materials : official publication of the Academy of Dental Materials. Mar 2017;33(3):321-332.

227. Shin JH, Kim JH, Koh JT, et al. Preparation and Characterisation of Hydroxyapatite Coatings on Nanotubular $\mathrm{TiO} 2$ Surface Obtained by Sol-Gel Process. Journal of nanoscience and nanotechnology. Aug 2015;15(8):6164-6167.

228. Abrishamchian A, Hooshmand T, Mohammadi M, Najafi F. Preparation and characterization of multi-walled carbon 
nanotube/hydroxyapatite nanocomposite film dip coated on Ti-6Al-4V by sol-gel method for biomedical applications: an in vitro study. Materials science \& engineering. C, Materials for biological applications. May 01 2013;33(4):2002-2010.

229. Family R, Solati-Hashjin M, Namjoy Nik S, Nemati A. Surface modification for titanium implants by hydroxyapatite nanocomposite. Caspian journal of internal medicine. Summer 2012;3(3):460-465.

230. Albrektsson T. Hydroxyapatite-coated implants: a case against their use. Journal of oral and maxillofacial surgery : official journal of the American Association of Oral and Maxillofacial Surgeons. Nov 1998;56(11):1312-1326.

231. Reigstad O, Franke-Stenport V, Johansson CB, Wennerberg A, Rokkum M, Reigstad A. Improved bone ingrowth and fixation with a thin calcium phosphate coating intended for complete resorption. Journal of biomedical materials research. Part B, Applied biomaterials. Oct 2007;83(1):9-15.

232. Albrektsson T, Åstrand P, Becker W, et al. Histologic studies of failed dental implants: A retrieval analysis of four different oral implant designs. Clinical Materials. 1992/01/01/ 1992;10(4):225-232.

233. Liao H, Fartash B, Li J. Stability of hydroxyapatite-coatings on titanium oral implants (IMZ). 2 retrieved cases. Clinical oral implants research. Feb 1997;8(1):68-72.

234. Hedia HS. Effect of coating thickness and its material on the stress distribution for dental implants. Journal of medical engineering \& technology. Jul-Aug 2007;31(4):280-287.

235. Skalak R, Zhao Y. Similarity of stress distribution in bone for various implant surface roughness heights of similar form. Clinical implant dentistry and related research. 2000;2(4):225230.

236. Meirelles L, Currie F, Jacobsson M, Albrektsson T, Wennerberg A. The effect of chemical and nanotopographical modifications on the early stages of osseointegration. The International journal of oral \& maxillofacial implants. Jul-Aug 2008;23(4):641-647.

237. Meirelles L, Arvidsson A, Andersson M, Kjellin P, Albrektsson T, Wennerberg A. Nano hydroxyapatite structures influence early bone formation. Journal of biomedical materials research. Part A. Nov 2008;87(2):299-307.

238. Jimbo R, Sotres J, Johansson C, Breding K, Currie F, Wennerberg A. The biological response to three different nanostructures applied on smooth implant surfaces. Clinical oral implants research. Jun 2012;23(6):706-712. 
239. Jimbo R, Coelho PG, Bryington M, et al. Nano hydroxyapatitecoated implants improve bone nanomechanical properties. Journal of dental research. Dec 2012;91(12):1172-1177.

240. Wennerberg A, Albrektsson T, Lausmaa J. Torque and histomorphometric evaluation of c.p. titanium screws blasted with 25- and 75-microns-sized particles of Al2O3. Journal of biomedical materials research. Feb 1996;30(2):251-260.

241. Sul YT, Johansson CB, Roser K, Albrektsson T. Qualitative and quantitative observations of bone tissue reactions to anodised implants. Biomaterials. Apr 2002;23(8):1809-1817.

242. Wennerberg A, Albrektsson T, Johansson C, Andersson B. Experimental study of turned and grit-blasted screw-shaped implants with special emphasis on effects of blasting material and surface topography. Biomaterials. Jan 1996;17(1):15-22.

243. Barkarmo S, Andersson M, Currie F, et al. Enhanced bone healing around nanohydroxyapatite-coated polyetheretherketone implants: An experimental study in rabbit bone. Journal of biomaterials applications. Nov 2014;29(5):737-747.

244. Yang Y, Kim K-H, Ong JL. A review on calcium phosphate coatings produced using a sputtering process-an alternative to plasma spraying. Biomaterials. 2005/01/01/ 2005;26(3):327337.

245. Nakahara I, Takao M, Bandoh S, Bertollo N, Walsh WR, Sugano N. In vivo implant fixation of carbon fiber-reinforced PEEK hip prostheses in an ovine model. Journal of orthopaedic research : official publication of the Orthopaedic Research Society. Mar 2013;31(3):485-492.

246. Nakahara I, Takao M, Goto T, Ohtsuki C, Hibino S, Sugano $\mathrm{N}$. Interfacial shear strength of bioactive-coated carbon fiber reinforced polyetheretherketone after in vivo implantation. Journal of orthopaedic research : official publication of the Orthopaedic Research Society. Oct 2012;30(10):1618-1625.

247. Durham JW, 3rd, Montelongo SA, Ong JL, Guda T, Allen MJ, Rabiei A. Hydroxyapatite coating on PEEK implants: Biomechanical and histological study in a rabbit model. Materials science \& engineering. C, Materials for biological applications. Nov 01 2016;68:723-731.

248. Durham JW, 3rd, Rabiei A. Deposition, Heat Treatment And Characterization of Two Layer Bioactive Coatings on Cylindrical PEEK. Surface \& coatings technology. Sep 15 2016;301:106-113.

249. Durham JW, Allen MJ, Rabiei A. Preparation, characterization and in vitro response of bioactive coatings on polyether ether 
ketone. Journal of Biomedical Materials Research Part B: Applied Biomaterials. 2017;105(3):560-567.

250. Kaygili O, Keser S, Kom M, Bulut N, Dorozhkin SV. The effect of simulating body fluid on the structural properties of hydroxyapatite synthesized in the presence of citric acid. Progress in biomaterials. Dec 2016;5(3-4):173-182.

251. Suska F, Omar O, Emanuelsson L, et al. Enhancement of CRFPEEK osseointegration by plasma-sprayed hydroxyapatite: A rabbit model. Journal of biomaterials applications. Feb 3 2014;29(2):234-242.

252. Lee JH, Jang HL, Lee KM, Baek HR, Jin K, Noh JH. Coldspray coating of hydroxyapatite on a three-dimensional polyetheretherketone implant and its biocompatibility evaluated by in vitro and in vivo minipig model. Journal of biomedical materials research. Part B, Applied biomaterials. Apr 2017;105(3):647-657.

253. Lee JH, Jang HL, Lee KM, et al. In vitro and in vivo evaluation of the bioactivity of hydroxyapatite-coated polyetheretherketone biocomposites created by cold spray technology. Acta biomaterialia. Dec 22012.

254. Johansson P. Biomechanical evaluation and surface characterization of a nano modified surface on PEEK implants: A study in the rabbit tibia. International journal of nanomedicine. 2014.

255. Webster TJ, Ergun C, Doremus RH, Siegel RW, Bizios R. Enhanced functions of osteoblasts on nanophase ceramics. Biomaterials. 2000/09/01/ 2000;21(17):1803-1810.

256. Zheng XB, Ding CX. Characterization of plasma-sprayed hydroxyapatite/TiO2 composite coatings. Journal of Thermal Spray Technology. December 01 2000;9(4):520-525.

257. Ramires PA, Romito A, Cosentino F, Milella E. The influence of titania/hydroxyapatite composite coatings on in vitro osteoblasts behaviour. Biomaterials. Jun 2001;22(12):14671474.

258. Li H, Khor KA, Cheang P. Thermal sprayed hydroxyapatite splats: nanostructures, pore formation mechanisms and TEM characterization. Biomaterials. 2004/08/01/ 2004;25(17):3463-3471.

259. Xiaolong Z, Oliver E, Christoph B, Lutz S, Jürgen G-G. Structural characterization of nanocrystalline hydroxyapatite and adhesion of pre-osteoblast cells. Nanotechnology. 2006;17(11):2711.

260. Wenzel RN. RESISTANCE OF SOLID SURFACES TO WETTING BY WATER. Industrial \& Engineering Chemistry. 
1936;28(8):988-994.

261. Kilpadi DV, Lemons JE. Surface energy characterization of unalloyed titanium implants. Journal of biomedical materials research. Dec 1994;28(12):1419-1425.

262. Ryden L, Omar O, Johansson A, Jimbo R, Palmquist A, Thomsen P. Inflammatory cell response to ultra-thin amorphous and crystalline hydroxyapatite surfaces. Journal of materials science. Materials in medicine. Jan 2017;28(1):9.

263. Hulander M, Lundgren A, Faxalv L, et al. Gradients in surface nanotopography used to study platelet adhesion and activation. Colloids and surfaces. B, Biointerfaces. Oct 01 2013;110:261269.

264. Ostman PO, Hellman M, Sennerby L. Direct implant loading in the edentulous maxilla using a bone density-adapted surgical protocol and primary implant stability criteria for inclusion. Clinical implant dentistry and related research. 2005;7 Suppl 1:S60-69.

265. Meredith N, Alleyne D, Cawley P. Quantitative determination of the stability of the implant-tissue interface using resonance frequency analysis. Clinical oral implants research. Sep 1996;7(3):261-267.

266. Christensen FB, Dalstra M, Sejling F, Overgaard S, Bunger C. Titanium-alloy enhances bone-pedicle screw fixation: mechanical and histomorphometrical results of titanium-alloy versus stainless steel. European spine journal : official publication of the European Spine Society, the European Spinal Deformity Society, and the European Section of the Cervical Spine Research Society. Apr 2000;9(2):97-103.

267. Spivak JM, Neuwirth MG, Labiak JJ, Kummer FJ, Ricci JL. Hydroxyapatite enhancement of posterior spinal instrumentation fixation. Spine. Apr 15 1994;19(8):955-964.

268. Szmukler-Moncler S. Evaluation of BONIT, a fully resorbable $\mathrm{CaP}$ coating obtained by electrochemical deposition after 6 weeks of heating : A pilot study in the pig maxilla. Biological Mechanisms of Tooth Eruption, Resorption, and Replacements by Implants. 19981998.

269. Sanden B, Olerud C, Larsson S. Hydroxyapatite coating enhances fixation of loaded pedicle screws: a mechanical in vivo study in sheep. European spine journal : official publication of the European Spine Society, the European Spinal Deformity Society, and the European Section of the Cervical Spine Research Society. Aug 2001;10(4):334-339.

270. Eom TG, Jeon GR, Jeong CM, et al. Experimental study of bone response to hydroxyapatite coating implants: bone- 
implant contact and removal torque test. Oral surgery, oral medicine, oral pathology and oral radiology. Oct 2012;114(4):411-418.

271. Johansson C, Albrektsson T. Integration of screw implants in the rabbit: a 1-year follow-up of removal torque of titanium implants. The International journal of oral \& maxillofacial implants. Spring 1987;2(2):69-75.

272. Galli S, Naito Y, Karlsson J, et al. Osteoconductive Potential of Mesoporous Titania Implant Surfaces Loaded with Magnesium: An Experimental Study in the Rabbit. Clinical implant dentistry and related research. Dec 2015;17(6):10481059.

273. Galli S, Andersson M, Jinno Y, et al. Magnesium release from mesoporous carriers on endosseus implants does not influence bone maturation at 6 weeks in rabbit bone. Journal of biomedical materials research. Part B, Applied biomaterials. Jul 132016.

274. Best S, Sim B, Kayser M, Downes S. The dependence of osteoblastic response on variations in the chemical composition and physical properties of hydroxyapatite. Journal of materials science. Materials in medicine. Feb 1997;8(2):97-103.

275. Noiset O, Schneider YJ, Marchand-Brynaert J. Fibronectin adsorption or/and covalent grafting on chemically modified PEEK film surfaces. Journal of biomaterials science. Polymer edition. 1999;10(6):657-677.

276. Lee JH. Quantitative Comparison of Novel CaO-SiO2-P2O5B2O3 Glass-Ceramics (BGS-7) with Hydroxyapatite as Bone Graft Extender in Rabbit Ilium. Tissue Engineering and Regenerative Medicine. 2010;7(5):540-547.

277. Wu GM, Hsiao WD, Kung SF. Investigation of hydroxyapatite coated polyether ether ketone composites by gas plasma sprays. Surface and Coatings Technology. 2009/06/15/ 2009;203(17):2755-2758.

278. Clarke B. Normal Bone Anatomy and Physiology. Clinical Journal of the American Society of Nephrology : CJASN. Nov 2008;3(Suppl 3):S131-139.

279. Meirelles L, Albrektsson T, Kjellin P, et al. Bone reaction to nano hydroxyapatite modified titanium implants placed in a gap-healing model. Journal of biomedical materials research. Part A. Dec 01 2008;87(3):624-631.

280. Stubinger S, Drechsler A, Burki A, Klein K, Kronen P, von Rechenberg B. Titanium and hydroxyapatite coating of polyetheretherketone and carbon fiber-reinforced polyetheretherketone: A pilot study in sheep. Journal of 
biomedical materials research. Part B, Applied biomaterials. Aug 2016;104(6):1182-1191.

281. Ripamonti U, Roden LC, Renton LF. Osteoinductive hydroxyapatite-coated titanium implants. Biomaterials. May 2012;33(15):3813-3823.

282. Dorland's Illustrated Medical Dictionary E-Book. Elsevier Health Sciences; 2011.

283. AlFarraj Aldosari A, Anil S, Alasqah M, Al Wazzan KA, Al Jetaily SA, Jansen JA. The influence of implant geometry and surface composition on bone response. Clinical oral implants research. Apr 2014;25(4):500-505.

284. Park JW, An CH, Jeong SH, Suh JY. Osseointegration of commercial microstructured titanium implants incorporating magnesium: a histomorphometric study in rabbit cancellous bone. Clinical oral implants research. Mar 2012;23(3):294300 .

285. Jimbo R, Anchieta R, Baldassarri M, et al. Histomorphometry and bone mechanical property evolution around different implant systems at early healing stages: an experimental study in dogs. Implant dentistry. Dec 2013;22(6):596-603.

286. Eriksson C, Nygren H, Ohlson K. Implantation of hydrophilic and hydrophobic titanium discs in rat tibia: cellular reactions on the surfaces during the first 3 weeks in bone. Biomaterials. 2004/08/01/ 2004;25(19):4759-4766.

287. Wilson CJ, Clegg RE, Leavesley DI, Pearcy MJ. Mediation of biomaterial-cell interactions by adsorbed proteins: a review. Tissue engineering. Jan-Feb 2005;11(1-2):1-18.

288. Andrade JD, Hlady V. Protein adsorption and materials biocompatibility: A tutorial review and suggested hypotheses. Biopolymers/Non-Exclusion HPLC. Berlin, Heidelberg: Springer Berlin Heidelberg; 1986:1-63.

289. Gittens RA, Scheideler L, Rupp F, et al. A review on the wettability of dental implant surfaces II: Biological and clinical aspects. Acta biomaterialia. 2014/07/01/ 2014;10(7):29072918.

290. Walsh WR, Pelletier MH, Bertollo N, Christou C, Tan C. Does PEEK/HA Enhance Bone Formation Compared With PEEK in a Sheep Cervical Fusion Model? Clinical orthopaedics and related research. Nov 2016;474(11):2364-2372.

291. Pelletier M, Cordaro N, Lau A, Walsh WR. PEEK Versus Ti Interbody Fusion Devices: Resultant Fusion, Bone Apposition, Initial and 26 Week Biomechanics. Journal of spinal disorders \& techniques. Jul 132012.

292. Hellbusch LC, Spangler WJ, Bowder A. Radiographic PEEK 
double-lucency finding after anterior cervical discectomy and fusion with local autograft and PEEK spacer: a preliminary study. Journal of neurosurgery. Spine. Mar 2012;16(3):248250.

293. Lee JH, Lee JH, Park JW, Lee HS. Fusion rates of a morselized local bone graft in polyetheretherketone cages in posterior lumbar interbody fusion by quantitative analysis using consecutive three-dimensional computed tomography scans. The spine journal : official journal of the North American Spine Society. Jul 2011;11(7):647-653.

294. Wendeberg B. Mineral metabolism of fractures of the tibia in man studied with external counting of Sr85. Acta orthopaedica Scandinavica. Supplementum. 1961;52:1-79.

295. Hayashi M, Jimbo R, Xue Y, Mustafa K, Andersson M, Wennerberg A. Photocatalytically induced hydrophilicity influences bone remodelling at longer healing periods: a rabbit study. Clinical oral implants research. Jun 2014;25(6):749754.

296. Ito M. Assessment of bone quality using micro-computed tomography (micro-CT) and synchrotron micro-CT. Journal of Bone and Mineral Metabolism. January 01 2005;23(1):115121.

297. Ma R, Yu Z, Tang S, Pan Y, Wei J, Tang T. Osseointegration of nanohydroxyapatite- or nano-calcium silicate-incorporated polyetheretherketone bioactive composites in vivo. International journal of nanomedicine. 2016;11:6023-6033.

298. Deng Y, Liu X, Xu A, et al. Effect of surface roughness on osteogenesis in vitro and osseointegration in vivo of carbon fiber-reinforced polyetheretherketone-nanohydroxyapatite composite. International journal of nanomedicine. 2015;10:1425-1447.

299. Marie PJ, Debiais F, Hay E. Regulation of human cranial osteoblast phenotype by FGF-2, FGFR-2 and BMP-2 signaling. Histology and histopathology. 2002;17(3):877-885.

300. Carlberg AL, Pucci B, Rallapalli R, Tuan RS, Hall DJ. Efficient chondrogenic differentiation of mesenchymal cells in micromass culture by retroviral gene transfer of BMP-2. Differentiation; research in biological diversity. Jun 2001;67(45):128-138.

301. Musgrave DS, Bosch P, Lee JY, et al. Ex vivo gene therapy to produce bone using different cell types. Clinical orthopaedics and related research. Sep 2000(378):290-305.

302. Vogt S, Wexel G, Tischer T, et al. The influence of the stable expression of BMP2 in fibrin clots on the remodelling and 
repair of osteochondral defects. Biomaterials. 2009/04/01/ 2009;30(12):2385-2392.

303. Wang W, Kratz K, Behl M, et al. The interaction of adiposederived human mesenchymal stem cells and polyether ether ketone. Clinical hemorheology and microcirculation. 2015;61(2):301-321.

304. Viguet-Carrin S, Garnero P, Delmas PD. The role of collagen in bone strength. Osteoporosis International. March 01 2006;17(3):319-336.

305. Ottani V, Raspanti M, Ruggeri A. Collagen structure and functional implications. Micron. Apr 2001;32(3):251-260.

306. Marotti G, Muglia MA, Palumbo C. Structure and function of lamellar bone. Clinical rheumatology. Dec 1994;13 Suppl 1:6368.

307. Hert J, Fiala P, Petrtyl M. Osteon orientation of the diaphysis of the long bones in man. Bone. May-Jun 1994;15(3):269-277.

308. Jones JI, Clemmons DR. Insulin-like growth factors and their binding proteins: biological actions. Endocrine reviews. Feb 1995;16(1):3-34.

309. Giustina A, Mazziotti G, Canalis E. Growth hormone, insulinlike growth factors, and the skeleton. Endocrine reviews. Aug 2008;29(5):535-559.

310. Takarada T, Hinoi E, Nakazato R, et al. An analysis of skeletal development in osteoblast-specific and chondrocyte-specific runt-related transcription factor-2 (Runx2) knockout mice. Journal of bone and mineral research : the official journal of the American Society for Bone and Mineral Research. Oct 2013;28(10):2064-2069.

311. Uskokovic V, Desai TA. In vitro analysis of nanoparticulate hydroxyapatite/chitosan composites as potential drug delivery platforms for the sustained release of antibiotics in the treatment of osteomyelitis. Journal of pharmaceutical sciences. Feb 2014;103(2):567-579.

312. He P, Sahoo S, Ng KS, Chen K, Toh SL, Goh JC. Enhanced osteoinductivity and osteoconductivity through hydroxyapatite coating of silk-based tissue-engineered ligament scaffold. Journal of biomedical materials research. Part A. Feb 2013;101(2):555-566.

313. Giachelli CM, Steitz S. Osteopontin: a versatile regulator of inflammation and biomineralization. Matrix biology : journal of the International Society for Matrix Biology. Dec 2000;19(7):615-622.

314. Hinoi E, Bialek P, Chen YT, et al. Runx2 inhibits chondrocyte proliferation and hypertrophy through its expression in the 
perichondrium. Genes \& development. Nov 01 2006;20(21):2937-2942.

315. Smith CJ, Osborn AM. Advantages and limitations of quantitative PCR (Q-PCR)-based approaches in microbial ecology. FEMS microbiology ecology. Jan 2009;67(1):6-20.

316. Zhu Y, Fang Q. Analytical detection techniques for droplet microfluidics--a review. Analytica chimica acta. Jul 17 2013;787:24-35.

317. Hanks CT, Wataha JC, Sun Z. In vitro models of biocompatibility: a review. Dental materials : official publication of the Academy of Dental Materials. May 1996;12(3):186-193.

318. Nahid M, Bottenberg P. [Importance of cell cultures in biocompatible dental materials research]. Revue belge de medecine dentaire. 2003;58(3):189-196.

319. Martiniakova M, Omelka R, Chrenek P, et al. Changes of femoral bone tissue microstructure in transgenic rabbits. Folia biologica. 2005;51(5):140-144.

320. Newman E, Turner AS, Wark JD. The potential of sheep for the study of osteopenia: current status and comparison with other animal models. Bone. Apr 1995;16(4 Suppl):277S-284S. 


\section{Endnotes figure legends}

I Blundell DJ, Osborn BN. The morphology of poly(arylether-ether-ketone). Polymer. 1983/08/01 1983;24(8):953958.

II Vaezi M, Yang S. Extrusion-based additive manufacturing of PEEK for biomedical applications. Virtual and Physical Prototyping. 2015/07/03 2015;10(3):123-135.

III Lin B, Yu H, Chen Z, Huang Z, Zhang W. Comparison of the PEEK cage and an autologous cage made from the lumbar spinous process and laminae in posterior lumbar interbody fusion. BMC musculoskeletal disorders. 08/30

IV Mobbs RJ, Phan K, Assem Y, Pelletier M, Walsh WR. Combination Ti/PEEK ALIF cage for anterior lumbar interbody fusion: Early clinical and radiological results. Journal of Clinical Neuroscience. 2016/12/01/ 2016;34(Supplement C):94-99.

V Brantigan JW, Steffee AD, Geiger JM. A carbon fiber implant to aid interbody lumbar fusion. Mechanical testing. Spine. Jun 1991;16(6 Suppl):S277-282.

VI Nakahara I, Takao M, Bandoh S, Bertollo N, Walsh WR, Sugano N. In vivo implant fixation of carbon fiberreinforced PEEK hip prostheses in an ovine model. Journal of orthopaedic research : official publication of the Orthopaedic Research Society. Mar 2013;31(3):485-492.

VII Converse GL, Yue W, Roeder RK. Processing and tensile properties of hydroxyapatite-whisker-reinforced polyetheretherketone. Biomaterials. Feb 2007;28(6):927935.

VIII Zhao Y, Wong HM, Wang W, et al. Cytocompatibility, osseointegration, and bioactivity of three-dimensional porous and nanostructured network on polyetheretherketone. Biomaterials. Dec 2013;34(37):92649277. 
IX Han CM, Lee EJ, Kim HE, et al. The electron beam deposition of titanium on polyetheretherketone (PEEK) and the resulting enhanced biological properties. Biomaterials. May 2010;31(13):3465-3470.

X Kjellin, P.; Andersson, M. Synthetic Nano-Sized Crystalline Calcium Phosphate and Method of

Production. US Pantent 8206813 B2, 26 June 2012.

XI Barkarmo S, Wennerberg A, Hoffman $M$, et al. Nanohydroxyapatite-coated PEEK implants: a pilot study in rabbit bone. Journal of biomedical materials research. Part A. Feb 2013;101(2):465-471.

XII Deng QX, Ou YS, Zhu Y, et al. Clinical outcomes of two types of cages used in transforaminal lumbar interbody fusion for the treatment of degenerative lumbar diseases: $\mathrm{n}$ HA/PA66 cages versus PEEK cages. Journal of materials science. Materials in medicine. Jun 2016;27(6):102. 
I 



\section{Biomechanical evaluation and surface characterization of a nano-modified surface on PEEK implants: a study in the rabbit tibia}

\author{
Pär Johansson' \\ Ryo Jimbo' \\ Per Kjellin ${ }^{2}$ \\ Fredrik Currie ${ }^{2}$ \\ Bruno Ramos Chrcanovic' \\ Ann Wennerberg'
}

'Department of Prosthodontics, Faculty of Odontology, Malmö University, Malmö, Sweden; ${ }^{2}$ Promimic AB, Göteborg, Swede

\begin{abstract}
Polyether ether ketone (PEEK) is today frequently used as a biomaterial in different medical operations due to its excellent mechanical and chemical properties. However, the untreated surface of PEEK is bioinert and hydrophobic, and it does not osseointegrate in its pure form. The aim of this study was to evaluate a unique nano-modified surface of PEEK with respect to osseointegration. Forty-eight threaded, non-cutting PEEK implants were inserted bilaterally in the tibia of 24 rabbits. Half of the implants $(n=24)$ were coated with nanocrystalline hydroxyapatite (test) and the remaining implants $(n=24)$ were left uncoated (control). Half of the animals $(n=12)$ were euthanized after 3 weeks of healing and the remaining $(n=12)$ after 12 weeks. The implant retention was measured with a removal torque apparatus. Surface analysis was performed with interferometry, scanning electron microscopy, and X-ray photon spectroscopy to relate the removal torque to the applied surface. The test implants revealed a significantly higher retention after 3 weeks $(P=0.05)$ and 12 weeks $(P=0.028)$ compared to controls. The result of the present study proves that the addition of nanocrystalline hydroxyapatite coating to PEEK surfaces significantly increases its removal torque and biocompatibility.

Keywords: polyether ether ketone, hydroxyapatite, removal torque, nanotopography
\end{abstract}

\section{Introduction}

Polyether ether ketone (PEEK) is a semi-crystalline thermoplastic material that has been used in situations where robustness and chemical resistance at high temperatures is required. In addition to its excellent mechanical properties, PEEK is chemically inert and resistant to sterilization. ${ }^{1-8}$ These features may be of great advantage for a biomaterial. PEEK has been used in orthopedic applications for decades and in the late 1990 s, became widely used as a substitute for metal implants in spinal surgery. Due to PEEK's translucency to X-rays, radiographic evaluation is more accessible and precise, which simplifies the postoperative evaluation and decisions for further treatment. ${ }^{7,9}$ By reinforcing PEEK with carbon fibers, the elastic modulus can be approximated to that of the cortical bone, which has been suggested to decrease stress shielding after spinal surgery compared to metal implants., ${ }^{40-14}$

The untreated surface of PEEK is bioinert and hydrophobic and it does not osseointegrate. $^{5}$ To convert the PEEK surface to be hydrophilic and osteoconductive, different techniques have been evaluated. It has been reported that by applying hydroxyapatite (HA) to PEEK, either mixed with the polymer or applied onto its surface, PEEK becomes more hydrophilic and possesses bioactivity. ${ }^{15,16}$ HA has been found to be an excellent coating material for enhanced osseointegration and previous studies have shown a significantly increased rate of bone formation compared to untreated surfaces. ${ }^{17-20}$ The modification converts the PEEK from bioinert 
to bioactive, since the synthesized HA bound to the implant surface blends into the natural HA in the bone. ${ }^{21} \mathrm{~A}$ commonly applied surface-coating method is the plasma spray technique. Plasma-sprayed HA implants have been found to significantly enhance and accelerate the early stages of bone formation. ${ }^{22}$ Furthermore, the effect of plasma-sprayed coating has been notable in situations where a gap exists between the implant and the bone, in which plasma-sprayed coats compensated for this gap and promoted further bone regeneration. ${ }^{23}$ However, clinical long-term complications with the use of plasma-sprayed HA have been documented. ${ }^{24}$ Rokkum et al found that the use of some thick-layered apatitecoated implants resulted in severe inflammation and bone resorption due to detachment of the coating material. ${ }^{25}$ It was identified histologically that multinucleated giant cells were localized in the proximity of the implant, and many of the cells resided around the detached HA particles. Registad et al have shown that plasma HA coating presents a gradual decrease in biomechanical fixation, which was observed for up to 52 weeks in a rabbit tibia. Furthermore, this histologic observation presented HA flake detachment and multinucleated giant cell infiltration in the proximity of the implant. ${ }^{26}$

To utilize the excellent bioactive properties of HA and to suppress the negative responses of thick HA layers, such as the detachment of the particles and osteoclastic reactions, a thinner and rigid HA coating may be desirable. With a thin HA coating, it is possible to retain the micro roughness of the implant substrate. We have previously shown in several studies that a thin layer of nanostructured HA obtained by a wet chemical-based technique may significantly enhance osseointegration..$^{27,28}$ With a thickness of 10-20 nm and HA crystals with similar size and shape as those found in human bone, it was suggested that the novel coating facilitates implant integration. ${ }^{27,29,30}$ Furthermore, since the nanosized HA coating is a monolayer, the risk of detachment is hypothesized to be lower than that of the thicker HA coatings. A recently published study, using an identical HA coating as in the present study, revealed a higher mean bone-to-implant contact for HA, indicating a higher level of osseointegration. ${ }^{31}$ Conversely, due to an unfavorable implant design, a large number of implants were lost due to a lack of primary stability. The design of the implant in the present study has taken into account the result of the previous study in order to achieve better primary stability. Therefore, the implant was provided with non-cutting threads to increase its primary stability. Instead of a pin-shaped implant with the flat top outside the cortical bone, the implant was redesigned to be fully submerged into the bone.
Due to the improved bioactivity and stable HA nanocoating around the PEEK material, it was hypothesized that the interfacial bonding strength would be significantly higher for the nano HA-coated implant surface compared to the non-coated PEEK material. Thus, the aim of the present study was to investigate the effect of a nano HA coating on PEEK implants placed in rabbit tibias after 3 and 12 weeks in vivo.

\section{Materials and methods Implant preparation}

Forty-eight threaded, non-cutting PEEK implants of $4 \mathrm{~mm}$ length and $3.5 \mathrm{~mm}$ diameter were manufactured for the study (Invibio Ltd., Thornton-Cleveleys, UK; Figure 1). Half of the implants $(\mathrm{n}=24)$ were coated with $\mathrm{HA}^{\text {nano }}$ Surface (test; Promimic AB, Göteborg, Sweden) and the remaining $(n=24)$ implants were left uncoated (control). For the implants to be coated, $50 \mu \mathrm{L}$ of coating solution was applied onto the top of each implant, and the implant was allowed to rotate at $2,700 \mathrm{rpm}$ for 5 seconds. The coating solution contains nanosized HA crystals that have been suspended with surfactants and solvent. The crystals are $20-50 \mathrm{~nm}$ long and 2-10 nm wide. After the coating step, the PEEK implant was put into an oven with an oxygen-enriched atmosphere at $325^{\circ} \mathrm{C}$ for 5 minutes. This heat treatment was done in order to remove
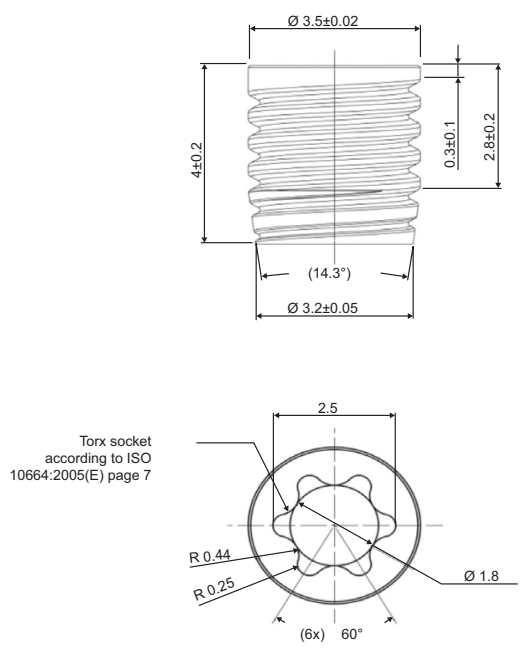

Figure I Technical drawing of a PEEK implant.

Abbreviations: ISO, International Organization for Standardization; PEEK, polyether ether ketone. 
the stabilizing surfactants and to attach the HA crystals onto the PEEK substrate.

\section{Surface characterization Interferometry}

The surface topography at the micrometer level was characterized with an interferometer (MicroXAM; ADE Phase Shift, Tucson, AZ, USA). The selected measurement area was $200 \times 260 \mu \mathrm{m}(\times 50$ objective, zoom factor 0.625$)$. A Gaussian filter of $50 \times 50 \mu \mathrm{m}$ was applied for removal of errors of form and waviness. Three samples from each group were measured $(n=6)$ each on three tops, flanks, and valleys of the threads (nine measurements per implant) according to guidelines by Wennerberg and Albrektsson. ${ }^{32}$ The following topographical parameters were selected for surface characterization: $\mathrm{S}_{\mathrm{a}}$ $(\mu \mathrm{m})=$ average deviation in height in relation to a prenominal plane; $\mathrm{S}_{\mathrm{ds}}\left(\mu \mathrm{m}^{-2}\right)=$ density of summits, a spatial parameter; and $\mathrm{S}_{\mathrm{dr}}(\%)=$ the developed surface ratio to a flat surface

\section{Atomic force microscopy (AFM)}

The surface topography at the nanometer level was characterized with an AFM (XE-100; Park Systems, Suwon, South Korea). Measurements were performed on discs since the microscope provides better resolution when used on a flat surface compared to a cylindrical one. Three discs of each PEEK group were analyzed on three random positions $(n=6)$. Measurements were performed in a non-contact mode at room temperature with two scan areas, $1 \times 1 \mu \mathrm{m}$ and $10 \times 10 \mu \mathrm{m}$. After leveling by subtraction, a Gaussian filter was applied $(0.25 \mu \mathrm{m}$ and $1 \mu \mathrm{m}$, respectively). Graphic 3D images were processed with the imaging software MountainsMap (Digital Surf, Besançon, France).

Scanning electron microscopy (SEM)

Illustrative images of the surface were obtained with an SEM (LEO Ultra 55 FEG; Zeiss, Oberkochen, Germany). The implants were sputtered with gold prior to analysis to make the surface conductive. Micrographs were obtained at two magnifications $(10 \mathrm{~K}$ and $80 \mathrm{~K}$ ) at diverse selected areas.

\section{Chemical characterization}

\section{X-ray photon spectroscopy (XPS)}

Chemical assessment of the surface was performed with an XPS (PHI 5000C ESCA System; PerkinElmer Inc., Waltham, MA, USA). Spectra were obtained at an operating angle of $45^{\circ}$, at $200 \mathrm{~W}$ with an $\mathrm{Al} \mathrm{K}$-alpha excitation source. Two locations on each sample were analyzed; at the top and bottom of the implant screw. The mean values were calculated.

\section{Mechanical characterization}

Since heat treatment was involved in the coating of the implants, it was of interest to evaluate how the heat treatment affected the mechanical strength of the PEEK material. Mechanical testing was performed in accordance with the tensile testing standard, International Organization for Standardization (ISO) 527-2. Samples for the testing were provided by Invibio Ltd. The specimens were of type $1 \mathrm{~A}$ in the ISO 527 standard, with a total length of $200 \mathrm{~mm}$, a thickness of $4 \mathrm{~mm}$, and a midsection width of $10 \mathrm{~mm}$. The samples were heat-treated at $300^{\circ} \mathrm{C}$ and $350^{\circ} \mathrm{C}$ for 10 minutes, ie, a time twice as long as used when applying the HA coating. Five samples were tested at each temperature. The measurements were performed by Swerea IVF AB (Mölndal, Sweden). An MTS 20/M (MTS Systems Corporation, Eden Prairie, MN, USA) was used for each measurement.

\section{Contact angle measurements}

The contact angles of the test and control surfaces were measured with a DAT 1100 from FIBRO System (Hägersten, Sweden). Water (Type 1, 18.2 M 2 ) was used as a test liquid and the drop volume was $4 \mu \mathrm{L}$. The measurements were done on PEEK discs since contact angle measurements on threaded surfaces are difficult to interpret.

\section{Surgical procedure and removal torque (RTQ)}

The surgical protocol of the current study was approved by the Malmö/Lund, Sweden, Regional Animal Ethics Committee. Twenty-four Swedish lop-eared rabbits of mixed sex and with a mean weight of $4.1 \mathrm{~kg}$ were selected for the study. On the day of surgical intervention, after fasting, the animals were administered a dose of $0.15 \mathrm{~mL} / \mathrm{kg}$ medetomidine $(1 \mathrm{mg} / \mathrm{mL}$ Dormitor $^{\text {; }}$; Orion Pharma AB, Sollentuna, Sweden) and $0.35 \mathrm{~mL} / \mathrm{kg}$ ketamine hydrochloride $\left(50 \mathrm{mg} / \mathrm{mL}\right.$ Ketalar ${ }^{\mathbb{R}}$; Pfizer AB, Sollentuna, Sweden) via a vein in the ear. The lower extremities were shaved and washed with $70 \%$ ethanol (Solveco AB, Rosersberg, Sweden) and $5 \mathrm{mg} / \mathrm{mL}$ chlorhexidine (Fresenius Kabi AB, Bad Homburg, Germany). After injection of approximately $1 \mathrm{~mL}$ local anesthesia (Xylocaine; AstraZeneca AB, Södertälje, Sweden), an incision was made along the proximal tibial epiphyseal and the margo cranialis tibiae was exposed. Osteotomy was performed with a series of drills under external continuous irrigation until a final diameter of $3.2 \mathrm{~mm}$ was reached. Prior to implant insertion, the preparation site was tapped in order to minimize shear on implant and coating. The implants were implanted by hand 
until they were fully submerged into the bone. The soft tissues were closed in separate layers, fascia, and subcutaneously with bioresorbable sutures (Ethicon, Norderstedt, Germany). Analgesic buprenorphine hydrochloride $(0.5 \mathrm{~mL}$ Temgesic; Reckitt Benckiser, Slough, UK) was administrated for 3 days post-surgically. After 3 and 12 weeks of healing, the animals were euthanized with an overdose of sodium pentobarbital ( $60 \mathrm{mg} / \mathrm{mL}$; Apoteksbolaget AB, Stockholm, Sweden). The tibias were retrieved and the soft tissue was removed. In order to measure the RTQ, a digital torque meter (Tohnichi Mfg. Co. Ltd., Ota-Ku, Japan) was connected to the implant driver and then to the implant. The RTQ was measured by counterclockwise rotation.

\section{Statistical analysis}

Statistical analyses were carried out using SPSS Version 20 software (IBM Corporation, Armonk, NY, USA). The topographical values were analyzed with independent samples Student's $t$-tests. Values from the bilaterally RTQs were submitted to the non-parametric Wilcoxon signed-rank test. The level of statistical significance was set at 0.05 .

\section{Results}

\section{Surface characterization} Interferometry

The interferometry measurements generated threedimensional images as well as roughness values, which are presented in Table 1 . The test group revealed the highest $\mathrm{S}_{\mathrm{a}}$ and the lowest $\mathrm{S}_{\mathrm{dr}}$; however, without statistical significance ( $P=0.556, P=0.849$, respectively). The $\mathrm{S}_{\mathrm{ds}}$ was significantly higher in the control samples $(P=0.002)$. Both surfaces present a minimally rough appearance $\left(\mathrm{S}_{\mathrm{a}}: 0.5-1.0 \mu \mathrm{m}\right)$ according to a recognized surface classification of dental implants. ${ }^{33}$

\section{SEM}

Surface morphology from the SEM analysis is presented in Figure 2. The HA-coated implants presented a random

Table I Mean values for $S_{a}, S_{d r}$, and $S_{d s}( \pm S D)$ for topographical analyses of implants with interferometer and $P$-values for oneway ANOVA comparisons

\begin{tabular}{llll}
\hline Mean $\pm \mathbf{S D}$ & $\mathbf{S}_{\mathrm{a}}(\mu \mathrm{m})$ & $\mathbf{S}_{\mathrm{dr}}(\%)$ & $\mathbf{S}_{\mathrm{ds}}\left(\mu \mathbf{m}^{-2}\right)$ \\
\hline Control & $0.655(0.23 \mathrm{I})$ & $53.3(67.7)$ & $\mathrm{I} 85,532(38,873)$ \\
Test & $0.686(0.140)$ & $50.6(23.8)$ & $\mathrm{I} 57,794(20,78 \mathrm{I})$ \\
$P$-value & 0.556 & 0.849 & 0.002 \\
\hline
\end{tabular}

Notes: $\mathrm{n}=6 ; \mathrm{S}_{\mathrm{a}}(\mu \mathrm{m})=$ average deviation in height in relation to a prenominal plane; $\mathrm{S}_{\mathrm{ds}}\left(\mu \mathrm{m}^{-2}\right)=$ density of summits, a spatial parameter; and $\mathrm{S}_{\mathrm{dr}}(\%)=$ the developed surface ratio to a flat surface.

Abbreviations: ANOVA, analysis of variance; SD, standard deviation. arrangement of HA rods covering the surface at the nanoscale level, whilst the control surfaces were relatively smooth at the same magnification. At the micrometer level, both surfaces presented similar roughness. The turning process caused striations and irregularities, which can be seen on the control surface at the lower magnification. The HA coating on the test implants evens out this orientation of surface features.

\section{XPS}

The XPS spectra for the uncoated and coated implants are shown in Figures 3A and B, respectively. Table 2 shows the atomic concentrations calculated from the spectra. Surfaces of the control samples reveal the presence of carbon, oxygen, and low levels of unexpected contaminations. The test samples showed the presence of carbon and oxygen, but also calcium and phosphorus from the HA crystals. The test samples had higher oxygen content, most probably from the HA crystals, which have a theoretical oxygen atomic content of around $62 \%$. The carbon content on the test samples was lowered from $88.8 \%$ to $55.5 \%$, a logical effect since the carbon-rich PEEK surface becomes shielded by the HA crystals. The $\mathrm{Ca} / \mathrm{P}$ ratio was 1.41 , which differs from the theoretical $\mathrm{Ca} / \mathrm{P}$ ratio for $\mathrm{HA}$ of 1.67 . Due to the semiquantitative nature of XPS, the difference in $\mathrm{Ca} / \mathrm{P}$ is well within the error margin.

\section{Mechanical testing}

Figures $4 \mathrm{~A}-\mathrm{C}$ show the stress-strain curves obtained from tensile testing; as seen from these figures, the stress-strain performance for the samples are virtually identical. A summary of the tensile testing is presented in Table 3 . The elastic modulus was measured to be approximately $4.2 \mathrm{GPa}$, which is in the same range as specified by the PEEK manufacturer. From these data, it can be concluded that the heat treatment that is part of the coating procedure does not alter the mechanical properties of PEEK.

\section{Contact angle measurements}

Contact angles were measured on uncoated and HAcoated discs and were found to be $53^{\circ}$ (SD: 4.4) and $88^{\circ}$ (SD: 2.7), respectively.

\section{Surgical procedure and RTQ}

No signs of infection of the surgical sites were observed during or after the healing periods for all animals. No implants were lost, but one of the rabbits that had been operated on died before sacrifice for unknown reasons. 

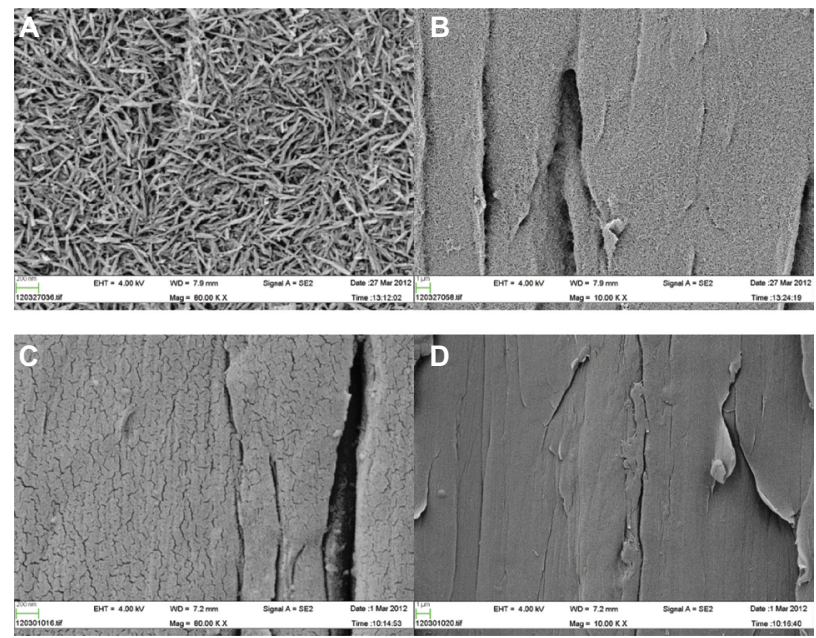

Figure 2 SEM images of HA-coated PEEK at (A) $80 \mathrm{k}$ magnification and (B) $10 \mathrm{k}$ magnification and uncoated PEEK at (C) $80 \mathrm{k}$ magnification and (D) $10 \mathrm{k}$ magnification. Abbreviations: SEM, scanning electron microscopy; PEEK, polyether ether ketone.

A

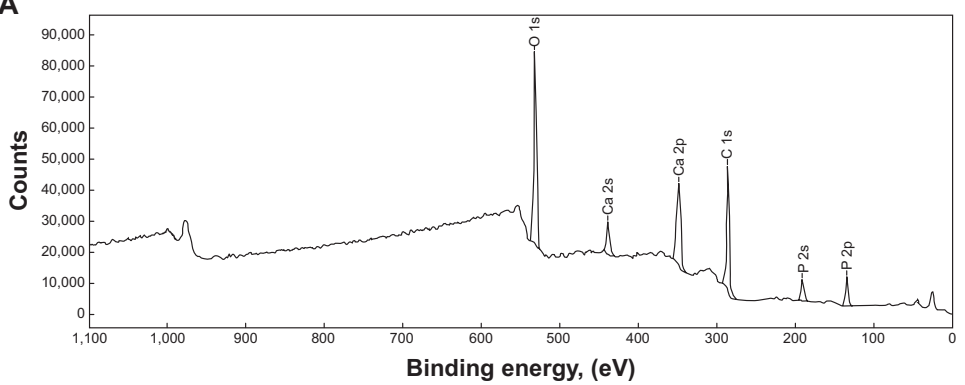

B

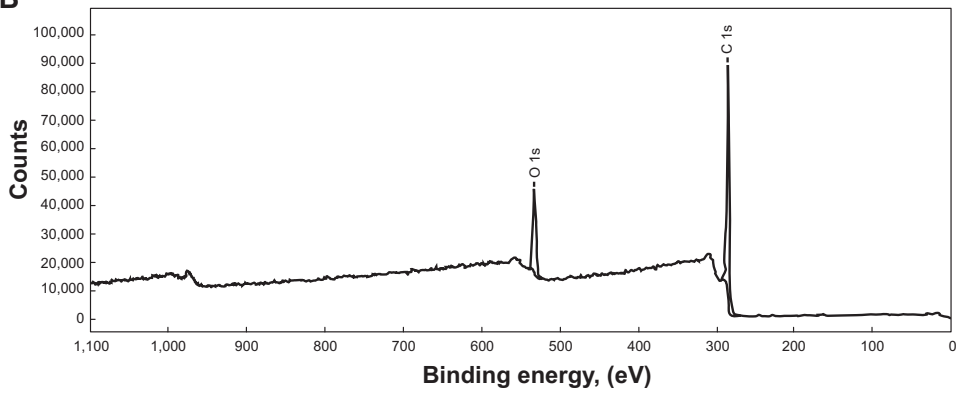

Figure 3 Typical XPS survey spectra of (A) HA-coated surface and (B) control surface.

Abbreviations: HA, hydroxyapatite; XPS, X-ray photon spectroscopy. 
Table 2 Elemental compositions from the XPS data of test and control surfaces

\begin{tabular}{lll}
\hline Atom & Test (\%) & Control (\%) \\
\hline $\mathrm{C}$ & 88.8 & 55.5 \\
$\mathrm{Ca}$ & 0 & 8.8 \\
$\mathrm{O}$ & $\mathrm{Il} .2$ & 29.5 \\
$\mathrm{P}$ & 0 & 6.2 \\
\hline
\end{tabular}

Note: Atomic concentration in percentage (\%).

Abbreviation: XPS, X-ray photon spectroscopy.

The mean and standard deviation of RTQ measurements for both groups are illustrated in Table 4 and Figure 5. The RTQ values for the control and test groups after 3 weeks of healing were $7.18 \mathrm{~N} \cdot \mathrm{cm}$ and $13 \mathrm{~N} \cdot \mathrm{cm}$, respectively. After 12 weeks of healing for the control and HA-coated groups, the RTQ values were $5.58 \mathrm{~N} \cdot \mathrm{cm}$ and $9.75 \mathrm{~N} \cdot \mathrm{cm}$, respectively. The mean values were higher for the test group after both healing periods ( 3 weeks: $P=0.008 ; 12$ weeks: $P=0.018$ ) and statistical analysis showed significant differences between the control and test implants for both evaluation times.

\section{Discussion}

The present study was performed to evaluate the effect of a nano HA coating on PEEK implants after 3 and 12 weeks of healing in rabbit tibia. Three weeks represents an early healing time and 12 weeks is when rapid increase in bone turnover healing is expected to have ceased. The unique feature of the test implants was the nanocrystalline surface with a $20 \mathrm{~nm}$ thick HA layer, which was hypothesized to promote the biocompatibility and bioactivity of the PEEK surface and further enhance and accelerate osseointegration. Furthermore, the thickness of the coated layer is believed to possess higher interfacial shear strength and in that way minimize the risk of coating delamination. A histological section, stained with toluidine blue, was cut from a deceased rabbit after 12 weeks of bone healing, and shows signs of new bone and several active osteocytes adjacent to the implant surface. There were no giant cells or similar indicating inflammatory response (Figure 6).
The mechanical testing showed that the heat treatment involved in the coating process did not affect the elastic modulus and the peak stress of the material. The heat treatment was carried out at $325^{\circ} \mathrm{C}$, which is well above the glass transition point of the polymer $\left(143^{\circ} \mathrm{C}\right)$ and close to its melting point $\left(343^{\circ} \mathrm{C}\right) .^{34}$ This may affect the crystallinity of the polymer and therefore also change its mechanical properties..$^{35}$ One explanation for the unchanged mechanical properties is probably the short duration of the heat treatment, heating at $325^{\circ} \mathrm{C}$ for longer time periods may well affect the mechanical stability.

The lower contact angle of the coated surfaces is not surprising since HA is a hydrophilic material. In comparison, previous measurements on rough $(\sim 1.5 \mu \mathrm{m}) \mathrm{HA}$-coated titanium have shown contact angles of around $10^{\circ}$. The reason for the higher contact angles on HA-coated PEEK could be that the surface was not fully covered with HA crystals, and/or an effect of the relatively smooth PEEK surface; the surface roughness of a substrate is known to affect the contact angle.

The mean RTQ values were significantly higher for test implants after both evaluation periods. The findings from the present study correlate with several previous studies where HA-coated titanium is evaluated in terms of osseointegration and RTQ. ${ }^{36-40}$ Yet, absolute RTQ values are highly distributed and it is difficult to compare one study to another due to implant design, surgical technique, animal species, and specific substrate material. Generally, the referred studies have found that retention significantly increases for titanium implants coated with HA after 2-6 weeks of healing. Furthermore, after a complete bone turnover, the retention has been found to be similar between the coated and uncoated samples. ${ }^{37}$ However, the finding of increased early retention of HA-coated titanium implants corresponds to the results from this study. ${ }^{37}$ It should be noted that these studies evaluated the retention of titanium implants, not PEEK. On the other hand, Nakahara et $\mathrm{al}^{40}$ examined the interfacial
A

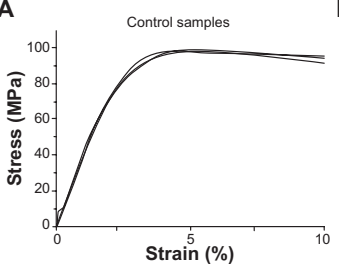

B

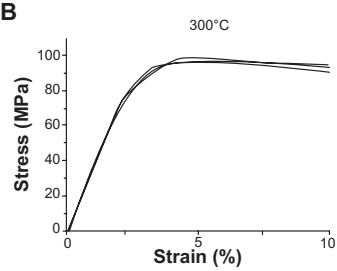

C

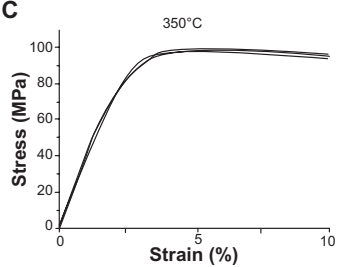

Figure 4 Stress-strain curves for (A) control samples with no heat treatment, (B) $300^{\circ} \mathrm{C}$, and (C) $350^{\circ} \mathrm{C}$. 
Table 3 Summary of tensile testing

\begin{tabular}{llllll}
\hline Sample & Peak load $(\mathbf{N})$ & Peak stress $\mathbf{( M P a )}$ & Modulus $\mathbf{( M P a )}$ & Strain at peak $(\%)$ & Break load $(\mathbf{N})$ \\
\hline Control & 3,997 & 98.0 & 4,211 & 4.943 & 3,418 \\
$300^{\circ} \mathrm{C}$ & 4,009 & 98.2 & 4,209 & 4.726 & 3,384 \\
$350^{\circ} \mathrm{C}$ & 4,050 & 99.2 & 4,184 & 4.839 & 3,390 \\
\hline
\end{tabular}

shear strength with an RTQ test of HA-coated PEEK and titanium implants. The test implants were cylindrical and HA granules were pressed onto the surface and exposed by blasting the surface with alumina beads. Unlike the present study, Nakahara et al evaluated osseointegration by pull-out removal instead of torque removal. Uncoated implants were used as the control and evaluation was completed after 6 and 12 weeks of healing. The RTQ test showed that HA-coated PEEK holds higher retention than all the other samples. The retention for HA-coated PEEK also increased after 12 weeks compared to 6 weeks, but not significantly. This indicates that the effect of HA coating is final at an earlier stage regardless of substrate material. In contrast, titanium showed increased interfacial shear at 12 weeks, which may be explained by bone-titanium integration. ${ }^{40}$

HA is an endogenous substance that can be found within osteoblast and osteoclast cells. After a cascade of actions in the cell, HA crystals will exit the cell and bind onto collagen molecules and initiate mineralization. ${ }^{41}$ By adding HA to an implant surface, mineralization may be stimulated at an early stage by forming new sites of initiation that can increase the amount and density of surrounding bone. Several studies have proven HA to be an effective coating substance to increase bone-screw interfaces. ${ }^{42,43}$ The size and shape of the deposited HA crystals in this study are similar to that in bone. Furthermore, nanoscale HA-coated implants may exceed micron-scale HA coatings in implant retention and histopathologic results. ${ }^{44}$ By incorporating HA onto the implant surface, not only are the chemical properties altered but also the surface topography. ${ }^{30}$ Surface topography can also be modified by exposing the surface to an accelerated neutral atom beam, and has successfully resulted in increased biocompatibility of PEEK. The authors explained that the increased biocompatibility was related to the surface nanometer texture, which

Table 4 Mean $\pm S D$ removal torque force $(N \cdot \mathrm{cm})$ for control and test groups

\begin{tabular}{lll}
\hline Mean \pm SD & 3 weeks & 12 weeks \\
\hline Control & $7.18(2.96)$ & $5.58(2.07)$ \\
Test & $13(5.2)$ & $9.75(4.65)$ \\
$P$-value & 0.05 & 0.028 \\
\hline
\end{tabular}

Note: $\mathrm{n}=23$.

Abbreviation: SD, standard deviation. enhanced cell attachment and proliferation. ${ }^{45}$ The results from this study correspond to the finding that nanoscale surface modification enhances RTQ and wettability, which together enhance the biocompatibility of PEEK. The numerical data on the micrometer level as evaluated with an interferometer revealed more dense peaks on the surface of the control group samples. However, the resolution of the interferometer is on the micrometer level and the applied surface modification is altering the surface at the nanoscale level. Barkarmo et al evaluated PEEK in vivo with the same rabbit model as in the present study. The surface properties of the coating and histomorphometry indicate that the HA-coated implants had more contact with bone $(16 \% \pm 4.7 \%$ vs $13 \% \pm 9.3 \%)$ and more bone area $(52 \% \pm 9.5 \%$ vs $45 \% \pm 11.9 \%)$. However, there were large numbers of lost implants due to loss of primary stability since the implants lacked threads. ${ }^{31}$ Therefore, the implant was redesigned and now possesses wide and shallow threads for primary stability to allow initial osseointegration. The implant was also designed to be fully submerged into the bone to minimize external loading during healing.

The results from the current study showed that the RTQ value was lower after 12 weeks when compared to 3 weeks for both test and control samples. A credible explanation for this tendency remains unknown. However, for the coated implants, a possible explanation may be the dissolution of HA particles from the surface, which has been previously studied in a rat model. ${ }^{46}$ Further studies are required to investigate the continued RTQ with a longer healing time to increase clinical relevance.

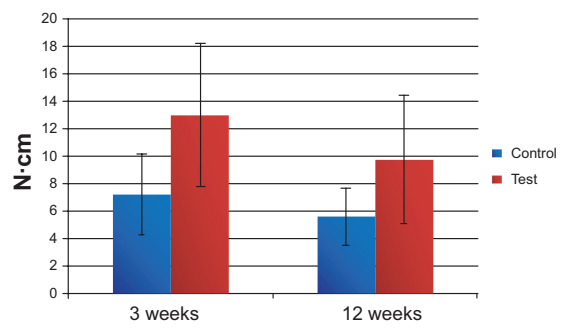

Figure 5 Mean values and standard deviation of removal torque $(\mathrm{N} \cdot \mathrm{cm})$ at both healing periods. 


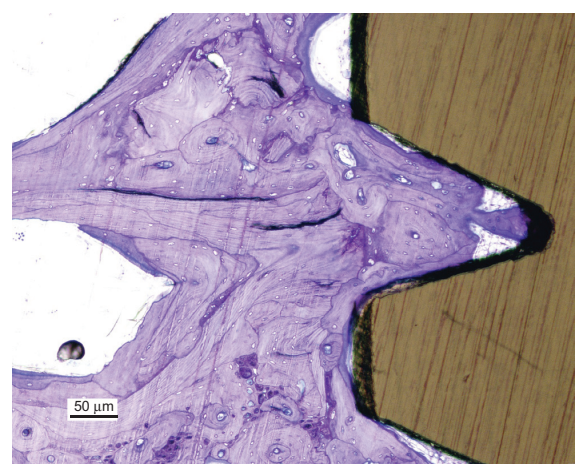

Figure 6 Histological section of the bone to implant interface, stained with toluidine blue.

\section{Conclusion}

The results of the present study indicate that the biocompatibility of PEEK can be improved with spin-coated nanosized HA. The application of the HA coating does not affect the mechanical strength of the PEEK. Evidence for this biocompatibility was derived from the increased RTQ. These results indicate that chemical inert materials such as PEEK can be converted into a suitable biomaterial for demanding orthopedic applications.

\section{Acknowledgment}

This study was supported by grants received from the Swedish Knowledge Foundation.

\section{Disclosure}

The authors report no conflicts of interest in this work.

\section{References}

1. Katzer A, Marquardt H, Westendorf J, Wening JV, von Foerster G Polyetheretherketone - cytotoxicity and mutagenicity in vitro. Biomaterials. 2002;23(8):1749-1759.

2. Ulrich D, Edwards SL, White JF, et al. A preclinical evaluation of alternative synthetic biomaterials for fascial defect repair using a rat abdominal hernia model. PloS One. 2012;7(11):e50044.

3. Williams D. Polyetheretherketone for long-term implantable devices. Med Device Technol. 2008;19(1):8, 10-11.

4. Toth JM, Wang M, Estes BT, Scifert JL, Seim HB 3rd, Turner AS. Polyetheretherketone as a biomaterial for spinal applications. Biomaterials. 2006;27(3):324-334.

5. Rabiei A, Sandukas S. Processing and evaluation of bioactive coatings on polymeric implants. J Biomed Mater Res A. 2013;101(9):2621-2629.

6. Nieminen T, Kallela I, Wuolijoki E, Kainulainen H, Hiidenheimo I, Rantala I. Amorphous and crystalline polyetheretherketone: mechanical properties and tissue reactions during a 3-year follow-up. J Biomed Mater Res A. 2008;84(2):377-383.

7. Kurtz SM, Devine JN. PEEK biomaterials in trauma, orthopedic, and spinal implants. Biomaterials. 2007;28(32):4845-4869.
8. Evans SL, Gregson PJ. Composite technology in load-bearing orthopaedic implants. Biomaterials. 1998;19(15):1329-1342.

9. Ponnappan RK, Serhan H, Zarda B, Patel R, Albert T, Vaccaro AR Biomechanical evaluation and comparison of polyetheretherketone rod system to traditional titanium rod fixation. Spine J. 2009;9(3): 263-267.

10. Walter J, Kuhn SA, Reichart R, Kalff R, Ewald C. PEEK cages as a potential alternative in the treatment of cervical spondylodiscitis: a preliminary report on a patient series. Eur Spine J. 2010;19(6):1004-1009.

11. Niu CC, Liao JC, Chen WJ, Chen LH. Outcomes of interbody fusion cages used in 1 and 2-levels anterior cervical discectomy and fusion: titanium cages versus polyetheretherketone (PEEK) cages. J Spinal Disord Tech. 2010;23(5):310-316.

12. Wang HR, Li XL, Dong J, Yuan FL, Zhou J. Skip-level anterior cervical discectomy and fusion with self-locking stand-alone PEEK cages for the treatment of two noncontiguous levels of cervical spondylosis. J Spinal Disord Tech. 2013;26(7):E286-E292.

13. Cutler AR, Siddiqui S, Mohan AL, Hillard VH, Cerabona F, Das K Comparison of polyetheretherketone cages with femoral cortical bone allograft as a single-piece interbody spacer in transforaminal lumbar interbody fusion. J Neurosurg Spine. 2006;5(6):534-539.

14. Kulkarni AG, Hee HT, Wong HK. Solis cage (PEEK) for anterior cervical fusion: preliminary radiological results with emphasis on fusion and subsidence. Spine J. 2007;7(2):205-209.

15. Wong KL, Wong CT, Liu WC, et al. Mechanical properties and in vitro response of strontium-containing hydroxyapatite/polyetheretherketone composites. Biomaterials. 2009;30(23-24):3810-3817.

16. Abu Bakar MS, Cheng MH, Tang SM, et al. Tensile properties, tension-tension fatigue and biological response of polyetheretherketonehydroxyapatite composites for load-bearing orthopedic implants. Biomaterials. 2003;24(13):2245-2250.

17. Yang GL, He FM, Song E, Hu JA, Wang XX, Zhao SF. In vivo comparison of bone formation on titanium implant surfaces coated with biomimetically deposited calcium phosphate or electrochemically deposited hydroxyapatite. Int J Oral Maxillofac Implants. 2010;25(4):669-680.

18. Park DS, Kim IS, Kim H, et al. Improved biocompatibility of hydroxyapatite thin film prepared by aerosol deposition. J Biomed Mater Res $B$ Appl Biomater. 2010;94(2):353-358.

19. Zhang H, Wang S, Zhao B. [Histocompatibility of porous hydroxyapatite coating NiTi shape memory alloy]. [Zhongguo xiu fu chong jian wai ke za zhi = Zhongguo xiufu chongjian waike zazhi]. Chin J Repar Reconstruct Surg. 2009;23(4):468-472. Chinese.

20. Hermida JC, Bergula A, Dimaano F, Hawkins M, Colwell CW Jr, D'Lima DD. An in vivo evaluation of bone response to three implant surfaces using a rabbit intramedullary rod model. J Orthop Surg Res. 2010;5:57.

21. Lee JH, Jang HL, Lee KM, et al. In vitro and in vivo evaluation of the bioactivity of hydroxyapatite-coated polyetheretherketone biocomposites created by cold spray technology. Acta biomater. 2013;9(4): 6177-6187.

22. Bonfante EA, Witek L, Tovar N, et al. Physicochemical characterization and in vivo evaluation of amorphous and partially crystalline calcium phosphate coatings fabricated on Ti-6Al-4V implants by the plasma spray method. Int J Biomater. 2012;2012:603826.

23. Manders PJ, Wolke JG, Jansen JA. Bone response adjacent to calcium phosphate electrostatic spray deposition coated implants: an experimental study in goats. Clin Oral Implants Res. 2006;17(5):548-553.

24. Albrektsson T. Hydroxyapatite-coated implants: a case against their use. J Oral Maxillofac Surg. 1998;56(11):1312-1326.

25. Røkkum M, Reigstad A, Johansson CB, Albrektsson T. Tissue reactions adjacent to well-fixed hydroxyapatite-coated acetabular cups. Histopathology of ten specimens retrieved at reoperation after 0.3 to 5.8 years. J Bone Joint Surg Br. 2003;85(3):440-447.

26. Reigstad O, Johansson C, Stenport V, Wennerberg A, Reigstad A, Røkkum M. Different patterns of bone fixation with hydroxyapatite and resorbable $\mathrm{CaP}$ coatings in the rabbit tibia at 6,12 , and 52 weeks. J Biomed Mater Res B Appl Biomater. 2011;99(1):14-20. 
27. Meirelles L, Arvidsson A, Andersson M, Kjellin P, Albrektsson T, Wennerberg A. Nano hydroxyapatite structures influence early bone formation. J Biomed Mater Res A. 2008;87(2):299-307.

28. Svanborg LM, Hoffman M, Andersson M, Currie F, Kjellin P, Wennerberg A. The effect of hydroxyapatite nanocrystals on early bone formation surrounding dental implants. Int J Oral Maxillofa Surg. 2011; 40(3):308-315.

29. Munir G, Koller G, Di Silvio L, Edirisinghe MJ, Bonfield W, Huang J. The pathway to intelligent implants: osteoblast response to nano silicon-doped hydroxyapatite patterning. JR Soc Interface. 2011;8(58) 678-688.

30. Meirelles L, Currie F, Jacobsson M, Albrektsson T, Wennerberg A. The effect of chemical and nanotopographical modifications on the early stages of osseointegration. Int J Oral Maxillofac Implants. 2008 23(4):641-647.

31. Barkarmo S, Wennerberg A, Hoffman M, et al. Nano-hydroxyapatitecoated PEEK implants: a pilot study in rabbit bone. J Biomed Mater Res A. 2013;101(2):465-471.

32. Wennerberg A, Albrektsson T. Suggested guidelines for the topographic evaluation of implant surfaces. Int J Oral Maxillofac Implants. 2000;15(3):331-344.

33. Albrektsson T, Wennerberg A. Oral implant surfaces: Part 1 - review focusing on topographic and chemical properties of different surfaces and in vivo responses to them. Int J Prosthodont. 2004;17(5):536-543.

34. Kurtz SM; Plastics Design Library. PEEK Biomaterials Handbook. 1st edition. Waltham, MA: William Andrew; 2012.

35. Hay JN, Langford JI, Lloyd JR. Variation in unit cell parameter of aromatic polymers with crystallization temperature. Polymer 1989;30(3):489-493.

36. Sandén B, Olerud C, Larsson S. Hydroxyapatite coating enhances fixation of loaded pedicle screws: a mechanical in vivo study in sheep. Eur Spine J. 2001;10(4):334-339.

37. Eom TG, Jeon GR, Jeong CM, et al. Experimental study of bone response to hydroxyapatite coating implants: bone-implant contact and removal torque test. Oral Surg Oral Med Oral Pathol Oral Radiol. $2012 ; 114(4): 411-418$
38. Cheng Z, Guo C, Dong W, He FM, Zhao SF, Yang GL. Effect of thin nano-hydroxyapatite coating on implant osseointegration in ovariectomized rats. Oral Surg Oral Med Oral Pathol Oral Radiol. 2012; 113(3):e48-e53.

39. Park YS, Yi KY, Lee IS, Han CH, Jung YC. The effects of ion beamassisted deposition of hydroxyapatite on the grit-blasted surface of endosseous implants in rabbit tibiae. Int J Oral Maxillofac Implants. 2005;20(1):31-38

40. Nakahara I, Takao M, Goto T, Ohtsuki C, Hibino S, Sugano N. Interfacial shear strength of bioactive-coated carbon fiber reinforced polyetheretherketone after in vivo implantation. J Orthop Res. 2012; 30(10):1618-1625.

41. Anderson HC. Molecular biology of matrix vesicles. Clin Orthop Relat Res. 1995;(314):266-280.

42. Yildirim OS, Aksakal B, Celik H, Vangolu Y, Okur A. An investigation of the effects of hydroxyapatite coatings on the fixation strength of cortical screws. Med Eng Phys. 2005;27(3):221-228.

43. Yildirim OS, Aksakal B, Hanyaloglu SC, Erdogan F, Okur A Hydroxyapatite dip coated and uncoated titanium poly-axial pedicle screws: an in vivo bovine model. Spine (Phila Pa 1976). 2006;31(8): E215-E220.

44. Aksakal B, Kom M, Tosun HB, Demirel M. Influence of micro- and nano-hydroxyapatite coatings on the osteointegration of metallic (Ti6Al4 V) and bioabsorbable interference screws: an in vivo study. Eur J Orthop Surg Traumatol. Epub 2013 May 21.

45. Khoury J, Kirkpatrick SR, Maxwell M, Cherian RE, Kirkpatrick A, Svrluga RC. Neutral atom beam technique enhances bioactivity of PEEK. Nucl Industr Meth B. 2013;(307):630-634.

46. Wennerberg A, Jimbo R, Allard S, Skarnemark G, Andersson M. In vivo stability of hydroxyapatite nanoparticles coated on titanium implant surfaces. Int J Oral Maxillofac Implants. 2011;26(6):1161-1166.

\section{Publish your work in this journal}

The International Journal of Nanomedicine is an international, peerreviewed journal focusing on the application of nanotechnolog in diagnostics, therapeutics, and drug delivery systems throughout he biomedical field. This journal is indexed on PubMed Central,

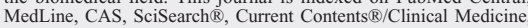

\section{Dovepress}

Journal Citation Reports/Science Edition, EMBase, Scopus and the Elsevier Bibliographic databases. The manuscript management system is completely online and includes a very quick and fair peer-review system, which is all easy to use. Visit http://www.dovepress.com/ testimonials.php to read real quotes from published authors.

Submit your manuscript here: htct://www,dovepress.com/international-journal-of-nanomedicine-journal 

II 

Article

\title{
Nanosized Hydroxyapatite Coating on PEEK Implants Enhances Early Bone Formation: A Histological and Three-Dimensional Investigation in Rabbit Bone
}

\author{
Pär Johansson ${ }^{1, *}$, Ryo Jimbo ${ }^{1}$, Yusuke Kozai ${ }^{2}$, Takashi Sakurai ${ }^{2}$, Per Kjellin ${ }^{3}$, Fredrik Currie ${ }^{3}$ \\ and Ann Wennerberg ${ }^{1}$ \\ 1 Department of Prostodontics, Faculty of Odontology, Malmö University, Malmö S-214 21, Sweden; \\ E-Mails: ryo.jimbo@mah.se (R.J.); ann.wennerberg@mah.se (A.W.) \\ 2 Department of Radiopraxis Science, Kanagawa Dental University, Yokosuka, \\ Kanagawa Prefecture 238-8580, Japan; E-Mails: kozai@kdu.ac.jp (Y.K.); \\ sakurait@kdcnet.ac.jp (T.S.) \\ 3 Promimic AB, Stena Center 1B, Göteborg S-412 92, Sweden; \\ E-Mails: per@promimic.se (P.K.); fredrik@promimic.se (F.C.) \\ * Author to whom correspondence should be addressed; E-Mail: par.johansson@mah.se; \\ Tel.: +46-40-665-85-14.
}

Academic Editor: Andrew J. Ruys

Received: 24 March 2015 / Accepted: 17 June 2015 / Published: 25 June 2015

\begin{abstract}
Polyether ether ketone (PEEK) has been frequently used in spinal surgery with good clinical results. The material has a low elastic modulus and is radiolucent. However, in oral implantology PEEK has displayed inferior ability to osseointegrate compared to titanium materials. One idea to reinforce PEEK would be to coat it with hydroxyapatite (HA), a ceramic material of good biocompatibility. In the present study we analyzed HA-coated PEEK tibial implants via histology and radiography when following up at 3 and 12 weeks. Of the 48 implants, 24 were HA-coated PEEK screws (test) and another 24 implants served as uncoated PEEK controls. HA-coated PEEK implants were always osseointegrated. The total bone area (BA) was higher for test compared to control implants at $3(p<0.05)$ and 12 weeks $(p<0.05)$. Mean bone implant contact (BIC) percentage was significantly higher $(p=0.024)$ for the test compared to control implants at 3 weeks and higher without statistical significance at 12 weeks. The effect of HA-coating was concluded to be significant with respect to early bone formation, and HA-coated PEEK implants may represent a good material to serve as bone anchored clinical devices.
\end{abstract}


Keywords: polyether ether ketone; HA; biomaterial; histology

\section{Introduction}

The search for the optimal replacement for human bone began with metallic devices in orthopedic, dental and trauma applications [1-3]. However, concern was raised regarding the release of toxic elements such as nickel and chromium due to corrosion. Furthermore a mechanical mismatch between bone and metallic implants was another concern. The stiffness of metal implants creates abnormal forces on the adjacent structures, sometimes resulting in stress shielding and segment degeneration $[4,5]$. Some of these unfavorable properties of metallic implants have been shown to cause osteolysis, allergenicity and implant detachment [6-8].

Today, the search for optimal implants continues with the ambition to compensate for adverse tissue reactions due to ionic leakage and mechanical mismatches. Polyether ether ketone (PEEK) has shown promising abilities as an alternative to metal implants due to its chemical resistance, mechanical properties and radiolucency [9]. PEEK has an elastic modulus between the value of cortical and cancellous bone, i.e., considerably lower than metal (PEEK: 3.2 GPa, CP Ti: $114 \mathrm{GPa}$, cortical bone: 15-30 GPa, cancellous bone: 0.5-1.5 GPa). Furthermore, PEEK may be machined or molded into any shape and size [6,9-13]. Regardless of these attributes, PEEK is relatively bio-inert and hydrophobic and has been found to osseointegrate poorly in its pure form $[9,14]$.

To increase the ability to osseointegrate, incorporation of hydroxyapatite (HA) into PEEK or onto its surface has been assessed. Although Ha-coating of PEEK has proven successful in increasing the bone incorporation, some studies have resulted in degraded mechanical abilities or reduced physical bonding between the substrate and the coated layer [15-19]. Deposition of a thin coating layer of HA on titanium implants has shown less degradation and dissolution [20]. In this study, PEEK implants were coated with HA using a unique procedure which creates a layer with a thickness measuring $20-40 \mathrm{~nm}$. This technique has previously been evaluated on titanium implants with promising outcomes [21,22]. The hypothesis of this work was that if crystalline HA was applied to a PEEK implant surface, the bone area in the threads and the bone-to-implant contact would be significantly larger than neat PEEK.

Thus, the overall purpose of this study was to investigate the biological effects of nano-HA coating on PEEK implants as evaluated by histomorphometry and micro-computed tomography. The study was conducted in a rabbit model and evaluated at 3 and 12 weeks after implantation.

\section{Results}

\subsection{Overall}

All animals healed uneventfully except one who died of unknown causes 1 week after implant insertion. At each retrieval point, all implants were immobile with no clinical signs of inflammation on the skin or around the surgical sites. 


\subsection{Coating Stability}

To reproduce the mechanical stress elicited when HA-coated and control PEEK implants were inserted in Sawbone material, we monitored the torque during insertion of the implants. Sawbone consists of a polyurethane foam with a filler, and is available in different porosities and densities, all designed to mimic cortico-cancellous bone proper. Monitoring the torque during the insertion gives an estimate on the mechanical forces on the implant surface.

As seen in Figure 1, the insertion torque profiles are virtually the same for coated and uncoated implants. Therefore, even if the coated and uncoated implants have different chemistries and nanostructures, the frictional forces seem most similar for the tested implants. This finding indicates that the friction was governed by the microtopography of the implant surface.

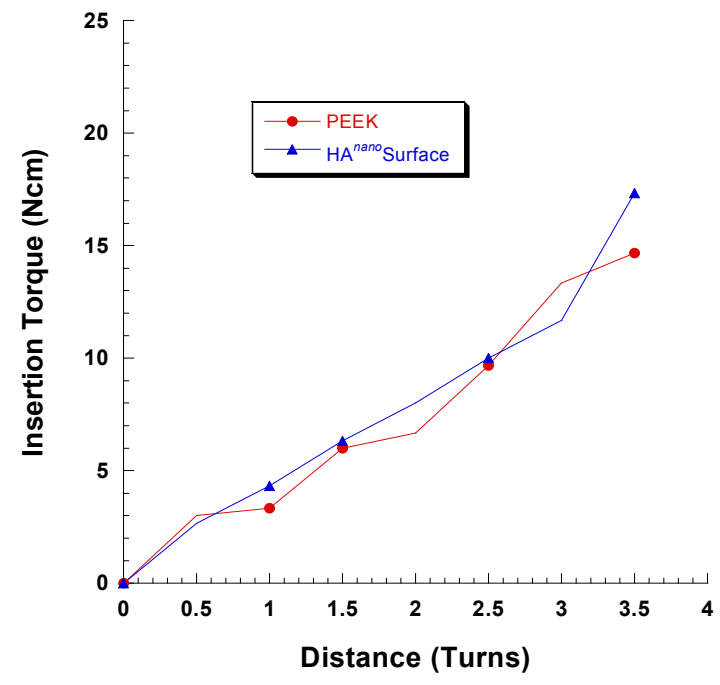

Figure 1. Insertion torque $v s$. distance for the coated and uncoated implants. The implant was inserted with its top at the level of the surrounding bone surface at 3.5 turns.

If the coating is to work properly in a clinical situation, it is important that the majority of the coating can withstand the mechanical forces which are created during the insertion process. Measuring the adhesion strength of the coating to the underlying surface gives valuable information for predicting the durability of the coating. However, standard adhesion measurement protocols for HA coatings such as tensile testing (for example ISO 13779-4 or ASTM F1147-05) are not suitable for coatings in the nanometer regime. These protocols have been suited for plasma sprayed micrometer-thick HA-coatings only. A qualitative assessment of the adhesion strength of the HA coating can be done by performing SEM analysis of the implants prior to and after insertion and removal of the implants.

As seen in Figure 2 the HA coating at the thread valleys was retained after the insertion and removal procedure. Also, along the thread "slopes," the coating was preserved. 


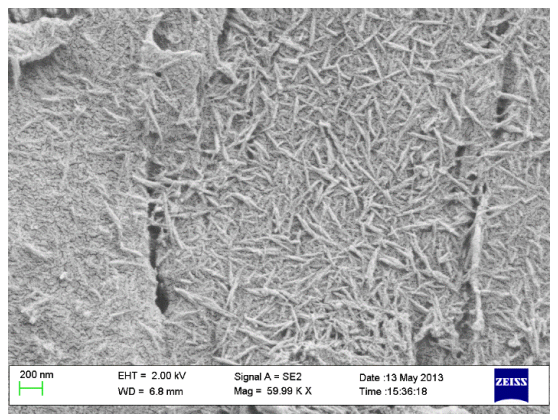

(A)

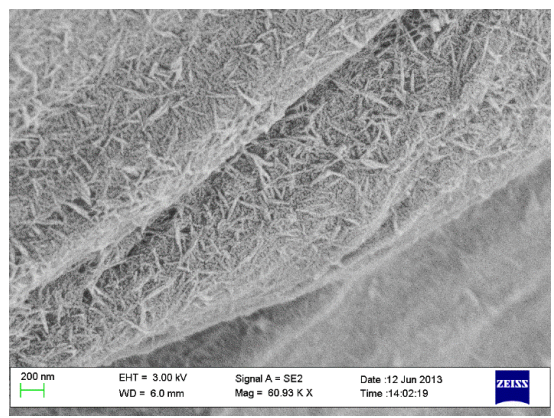

(B)

Figure 2. SEM images of a PEEK implant at the thread valleys (A) before insertion and (B) after insertion and removal. Scale bar $=200 \mathrm{~nm}$.

The thread edges are generally the parts which are subjected to the highest mechanical forces during placement and removal of the implant. As seen in Figure 3, the coating is well-preserved in the small cracks and fissures on the edges, but on the flat areas which were in direct contact with the Sawbone the PEEK surface showed signs of deformation which, in turn, may lead to low surface coverage of HA crystals.

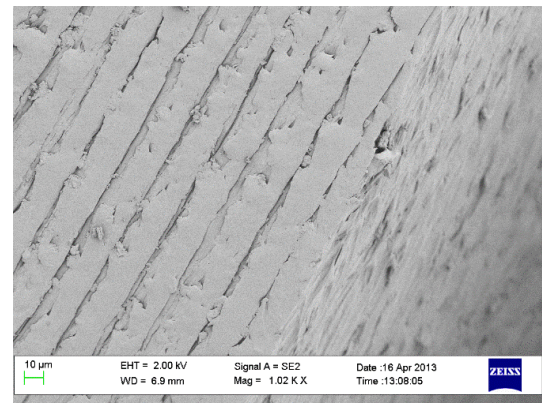

(A)

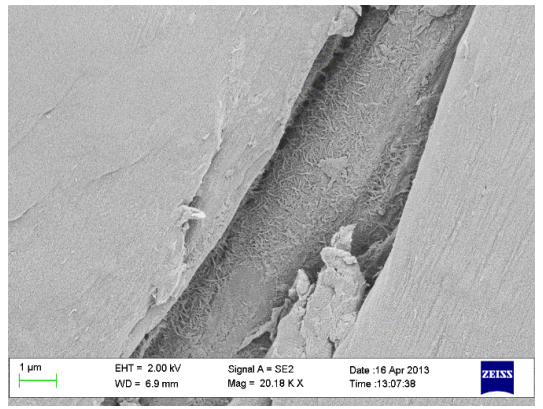

(B)

Figure 3. SEM images of thread edge of implant after insertion and removal. (A) scale bar $=10 \mu \mathrm{m}$ and (B) scale bar $=1 \mu \mathrm{m}$.

\subsection{Micro-CT Evaluation}

The results acquired from the micro computed tomographic evaluation showed for the full volume of interest (VOI, D1 + D2 + D3, Figure 4) no significant differences between the two groups after both 3 and 12 weeks of healing. The inner VOI (D1 closest to the implant thread) revealed, after 12 weeks only, a significant difference $(p=0.044)$ between the test and control when comparing bone surface in terms of volume (Test: $23.5 \% \pm 1.5 \%$, Control; $24.7 \% \pm 4.3 \%$ ) and trabecular thickness (Test: $85.5 \pm 5.4 \mu \mathrm{m}$, 
Control: $82.8 \pm 12.1 \mu \mathrm{m})$. This difference aside, there were no other significant differences between the groups or healing periods.

D1 D2 D3

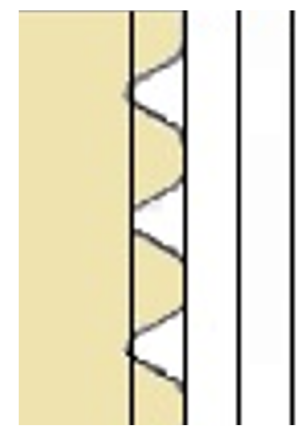

Figure 4. Schematic plan of the subdivision of micro-CT volume of interest (VOI). D1 is the closest to the threads and D3 is the one furthest away.

\subsection{Histomorphometry}

The histological sections presented homogenous blue colored staining adjacent to the implant surface of the two groups. In proximity to the surface coating there were no signs of detached coating debris or giant cells indicative of an inflammatory response. After 12 weeks most of the threads were filled with bone. Signs of osteoconductive features of HA-coated PEEK implants were observed (Figure 5).

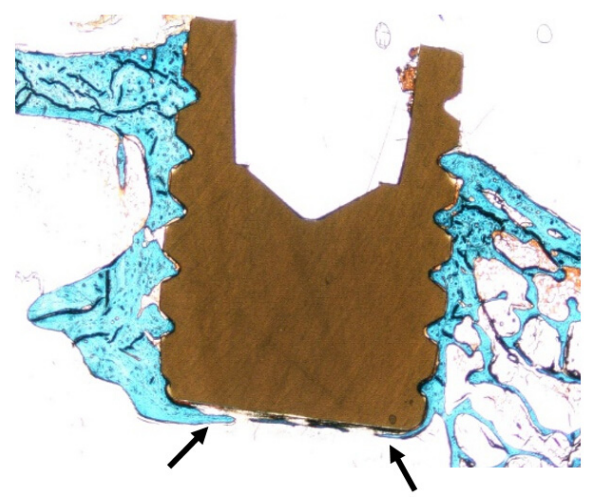

Figure 5. Histologic section of all threads presenting manifest bone ingrowth in all threads, indicative of osteoconductive features of the PEEK material (arrow). This actual section was from an HA-coated implant retrieved 12 weeks after surgery. 
The histological outcomes after 3 weeks of healing revealed significantly higher BA and BIC values for test implants compared to controls (BA: $p=0.02$, BIC: $p=0.024)$. Bone area (\%, SD) after 3 weeks for test and control was 27.5 (10.6) and 17.9 (5.8) respectively and the BIC (\%, SD) was test 7.94 (6.94) and control 2.91 (1.87, Figure 6A-D and Table 1).

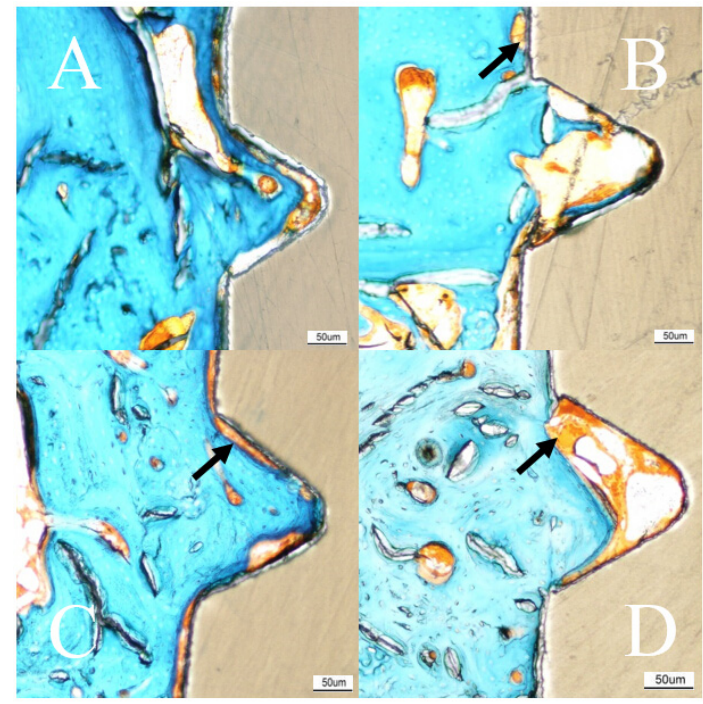

Figure 6. Histologic results. Masson Goldner-Trichrome stained sections of the implant after 3 and 12 weeks of healing. No soft tissue, or only sparse amounts (green), have migrated down between the implant and bone. Fibrous tissue (orange, arrow) can be viewed at the gap between the bone and implant, more commonly at the 3 week samples. (A) 3 weeks test (HA); (B) 3 weeks control; (C) 12 weeks test (HA); (D) 12 weeks control. Scale bar $=50 \mu \mathrm{m}$.

Table 1. Histomorphometric results 3 and 12 weeks after implantation.

\begin{tabular}{cccc}
\hline \multirow{2}{*}{$\boldsymbol{n}=\mathbf{2 4}$} & & \multicolumn{2}{c}{ Average (SD) } \\
\cline { 3 - 4 } & & $\begin{array}{c}\text { Bone-Implant Contact } \\
\text { (BIC, \%) }\end{array}$ & $\begin{array}{c}\text { Bone Area (BA, \%) } \\
\text { (in Thread) }\end{array}$ \\
\hline \multirow{3}{*}{ 3 weeks } & Test & $7.94(6.94)$ & $27.49(10.63)$ \\
& Control & $2.91(1.87)$ & $17.91(5.78)$ \\
& p-value & $\mathbf{0 . 0 1 6}$ & $\mathbf{0 . 0 2 7}$ \\
& Test & $6.75(6.52)$ & $48.72(11.12)$ \\
& Control & $4.34(3.28)$ & $38.27(9.43)$ \\
& p-value & 0.622 & 0.02 \\
\hline
\end{tabular}

The second healing point at 12 weeks showed higher BIC values for test implants compared to controls but without statistical significance. The BA (\%, SD) was for test 48.7 (11.1) and control 38.3 (9.4) 
after 12 weeks. The BIC (\%, SD) was measured to 6.75 (6.52) and 4.34 (3.28) for test and control, respectively (Figure 7).

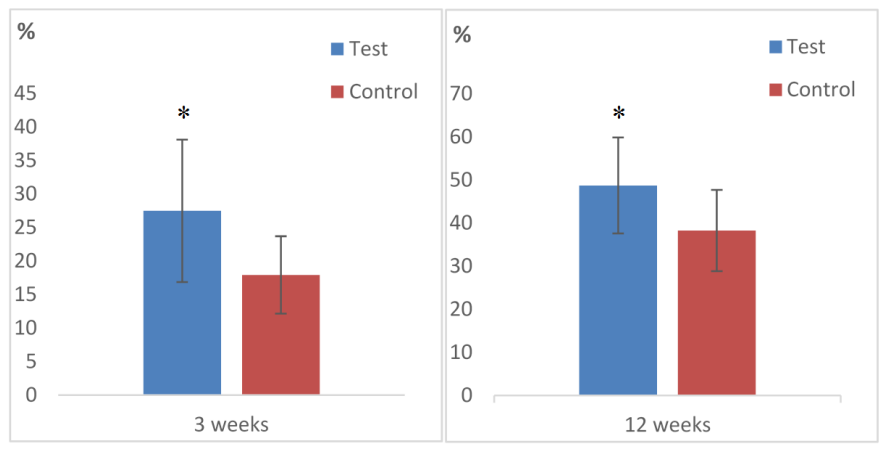

(A) Bone Area (BA)

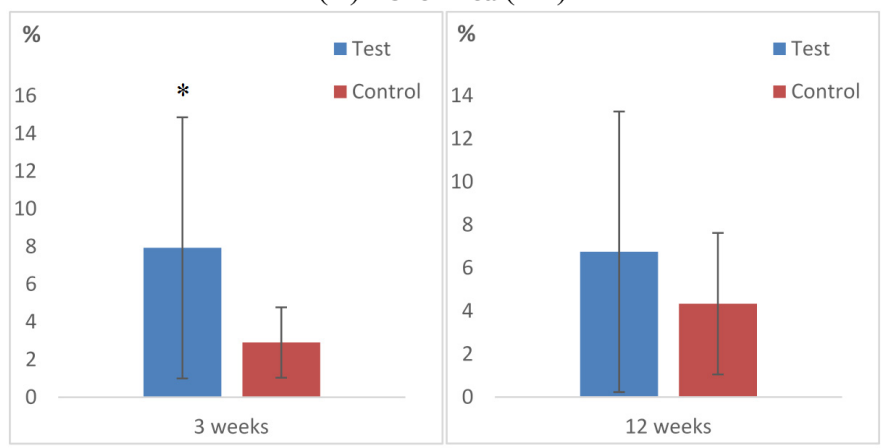

(B) Bone-Implant Contact (BIC)

Figure 7. Bar graphs displaying the percentage of (A) bone area (BA) and (B) bone-to-implant contact (BIC) for test and control after 3 and 12 weeks of healing. Data are presented as mean with standard deviation. Mann Whitney $\mathrm{U}$ test was used for testing level of significance. $(*, p \leq 0.05)$.

\section{Discussion}

A nano thin hydroxyapatite coating on PEEK implants was evaluated and found to increase the bone-implant contact. This increased BIC conforms to several other studies on hydroxyapatite coated titanium $[9,23]$.

The histologic results demonstrated bone-forming activity adjacent to the test in contrast to the control implants, since the histological BIC was significantly higher for the HA-coated PEEK implant after 3 weeks $(p=0.024)$. After 12 weeks of healing, the HA-coated implants still possessed higher BIC than the controls, but without significant difference. This trend has been confirmed in several 
previous studies evaluating thin coating layers [24-26]. Our finding with regard to a stronger bone reaction to test implants is supported by our previous biomechanical study on similar test and control implants [27]. In this previous study, the removal torque for 3 weeks was 13.0 and $7.18 \mathrm{Ncm}$, and for 12 weeks, 9.75 and $5.58 \mathrm{Ncm}$, for test and control implants, respectively. Taken together, the rate of new bone formation was enhanced for HA-coated implants in the early healing point, which also concurs with several previous reports [28-30]. Lee et al. evaluated different ceramic coatings and found that the attraction to bone is weaker with time after implantation [31]. Nonetheless, ceramic coatings differ from many metal implants, where the stability relies on the mechanical bonding created by the surface topography [32]. Having said this, with time the bone response is also weakened in metal implants [32].

The percentage of bone in contact with the uncoated control PEEK surface was surprisingly low. The BIC only increased from 2.91 percent to 4.94 percent from 3 to 12 weeks. This minor increase in BIC after 12 weeks of implantation was very different when compared to studies on machined titanium implants $[33,34]$. Barkamo et al. evaluated HA-coated and untreated PEEK implants after one healing period of six weeks. When measuring all threads they found significantly higher BIC for HA-coated PEEK but the BA was similar in both groups [35]. The finding of a BIC advantage for HA-coated PEEK was similar to this study, which also showed a significantly higher BA for HA-coated PEEK after 3 weeks. However, the absolute values cannot be compared due to the use of different drilling protocols, healing times and interpersonal perceptions of the histologic image. The removal torque results from the study of Barkamo et al. was comparable to our previous study [27], where HA-coated PEEK required significantly higher removal torque compared to untreated PEEK. Overall, the results from these studies are similar, and together they indicate the osteoinductive effect of an HA coating.

The surface of the test implant consisted of HA rods, nanometers in size and the overall topography was, in a previous study for both groups, classified as minimally rough $\left(\mathrm{S}^{\mathrm{a}} 0.5-1.0 \mu \mathrm{m}\right)$ somewhat below the optimal roughness for dental implants $\left(\mathrm{S}^{\mathrm{a}} 1.5 \mu \mathrm{m}\right)$ suggested by Albrektsson and Wennerberg [27,36]. Many studies have been conducted to evaluate whether nanotopography affects bone formation. Meirelles et al. evaluated HA-coated titanium implants with different nanotopographies in early stages of osseointegration with respect to removal torque and histologic measurements [37]. They found a correlation between chemical modification, nanotopography and increased removal torque. This study compared two groups with different chemical modification that did not change the surface topography (Test: $\mathrm{S}^{\mathrm{a}}=0.69 \mu \mathrm{m}$, control: $\mathrm{S}^{\mathrm{a}}=0.66 \mu \mathrm{m}$ ) [27]. Thus, only the influence of the HA coating was evaluated. The results from this and our previous study indicate, as did Meirelles et al., that removal torque, BIC and BA was increased with an HA coating of the surface. The decrease of BIC after 12 weeks can be compared with the decreased removal torque, which indicates the early impact of the coating but also the disadvantage of the smooth implant surface. When it comes to evaluating bone-implant integration, the results become a mixture of several mechanisms, which are still not fully identified. For instance, the morphological appearance alone may enhance the bone-implant interlocking regardless of surface chemical modification. Therefore, this study aimed to evaluate the level of osseointegration using histology and radiology, while our previous study focused on the mechanical bond through removal torque testing [27].

The quantitative assessment of BA within the threads revealed a clear and significantly increased area for test implants ( 3 weeks: $p=0.027,12$ weeks: $p=0.02$, Figures 6 and 7). A greater amount of 
bone was found within the threads even newly formed bone reached the apical part of the implant. This osteoinductive feature of HA is well supported by several other studies where HA have been applied to different implant materials [27,38,39]. However, the specific osteoinductive process of ceramic coatings is still unclear but authors have reported that surface roughness and chemical composition have decisive influence on the outcome [40-42]. Other studies have shown that the dissolution of HA ions has an up-regulating effect on the osteoblast activity, which may explain the in vivo results of the present paper [43]. The precise biological events remain unclear and are in need of further study. PEEK has a radiolucent feature that gives the surgeon an improved radiographic interpretation after spinal surgery [44]. Correspondingly, in experimental setups this feature gives the researcher many advantages. The evaluation can be performed in a non-invasive manner with an intact sample, and the bone-implant interface can be assessed three-dimensionally. Therefore, in this study we chose to perform a computed tomographic evaluation before the samples were cut and ground in order to obtain a detailed view of the interface. Micro-CT is a reliable non-invasive analytical instrument to quantify the amount of mineralized bone around artificial implants and characterize the bone structures $[45,46]$. Together with the histological sections, a comprehensive image of the bone can be surveyed and provide a representative description of the healing event. In this report, the micro-CT evaluation was focused on potential differences in mineralized bone volume and trabecular organization. BV/TV indicates the fraction of bone in the given VOI, which explains the effect of the bioactive coating. However, the present study revealed no differences between the groups. This could be explained by an inappropriate selection of VOI which makes the small bone changes undetectable, in addition to the voxel resolution of the radiographic equipment that could have limited the possibility to detect minor deviations.

\section{Experimental Section}

\subsection{Implant Manufacturing}

Commercial PEEK substrates (Invibio Ltd. Lancashire, UK) with non-cutting screw shape and diameter of $3.5 \mathrm{~mm}$ and length of $4 \mathrm{~mm}$ were prepared for the study by Elos Pinol A/S, Görlöse, Denmark (Figure 8).

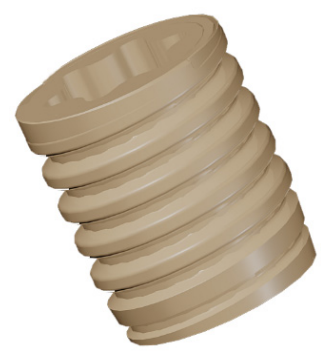

Figure 8. 3D rendered image of the PEEK implant. 


\subsection{Coating Preparation}

Altogether, 24 implants were coated with the $\mathrm{HA}^{\text {nano }}$ Surface (Promimic, Göteborg, Sweden (test)) whereas the remaining 24 implants were left uncoated (control). The coating solution was prepared according to patent US8,206,813 [47]. The coating solution consisted of $15 \mathrm{~g}$ water, nanocrystalline hydroxyapatite, $70 \mathrm{~g}$ of poly(ethylene oxide)-block-poly(propylene oxide)-block-poly(ethylene oxide) copolymer (Pluronic L64,(EO)13(PO)30(EO)13) and $205 \mathrm{~g}$ of p-xylene. The HA ${ }^{\text {nano }}$ surface was applied by adding $50 \mu \mathrm{L}$ of the coating solution followed by rotation at $2700 \mathrm{rpm}$ for $5 \mathrm{~s}$. After the coating procedure, the substrates were heated $\left(325^{\circ} \mathrm{C}\right.$ for $5 \mathrm{~min}$.) in an oxygen atmosphere to burn off the surfactants and to adhere the HA crystals on the PEEK substrate. The coating solution consisted of nanosized hydroxyapatite crystals (length $20-50 \mathrm{~nm}$ and width 2-10 $\mathrm{nm}$ ) which were dispersed with surfactants, water and organic solvent, as previously described [27].

\subsection{Coating Adhesion}

Sawbone 40 PCF was used as a model system to simulate the mechanical wear created by insertion. The insertion and removal torque of the implants was monitored with a Tohnichi BTG90CN torque gauge (Tohnichi America Corp, Buffalo Grove, IL, USA). Three coated and three uncoated implants were used for the measurement. Scanning electron microscopy (SEM) analysis revealed that residues of the Sawbone material remained on the implant surface after removal, these residues were removed by sonicating each implant in $15 \mathrm{~mL}$ ethylene diamine (purum, $\geq 99.0 \%$, Fluka) for $15 \mathrm{~min}$, using an ultrasonic cleaner (VWR USC100T, VWR, Leuven, Belgium). The implants were analyzed with a scanning electron microscope (SEM, LEO Ultra 55, Zeiss, Oberkochen, Germany) prior to and after the insertion testing.

\subsection{Morphological Characterization}

The surface morphology was evaluated using SEM (LEO Ultra 55 FEG, Zeiss, Oberkochen, Germany) at an accelerating voltage of 2-4 kV. To make the surface conductive, the implants were sputtered with gold in a Jeol JFC-1100E ion sputter (JEOL Ltd., Tokyo, Japan) at $10 \mathrm{~mA}$ for $90 \mathrm{~s}$. The nano- and microtopography was described in a previous report [27].

\subsection{Surgical Procedure and Implantation}

This experimental study was approved by Malmö/Lund, Sweden, Regional Animal Ethics Committee. Twenty-four lop-eared rabbits with mixed gender and an approximate mean weight of $4.1 \mathrm{~kg}$ (Range: 3.4-5.1 kg) were used for the study. The rabbits were divided into two groups, each consisting of 12 animals, for observation periods of either 3 or 12 weeks after implant surgery. Three weeks refers to the early stage of new bone formation which continues until week 12 where complete bone healing has occurred. The animals were administered a dose of $0.15 \mathrm{~mL} / \mathrm{kg}$ medetomidine $(1 \mathrm{mg} / \mathrm{mL}$ Dormitor; Orion Pharma, Sollentuna, Sweden) and $0.35 \mathrm{~mL} / \mathrm{kg}$ ketamine hydrochloride $(50 \mathrm{mg} / \mathrm{mL}$ Ketalar; Pfizer AB, Sollentuna, Sweden). After removing the fur, the skin of the intended surgical sites (Figure 9) was disinfected with $70 \%$ ethanol (Solveco AB, Rosersberg, Sweden) followed by $5 \mathrm{mg} / \mathrm{mL}$ chlorhexidine (Fresenius Kabi AB, Uppsala, Sweden). Local anesthesia with lidocaine hydrochloride 
(Xylocain, AstraZeneca AB, Södertälje, Sweden) was injected subcutaneously (approx. $1 \mathrm{~mL}$ ) to reduce the amount of blood at the surgical site. A skin incision was made over the tibia crest inferior to the joint. The tibia bone was exposed by separating the fascia and muscles. The implant sites were prepared with a series of drills up to a final diameter of $3.2 \mathrm{~mm}$. Tapping and insertion was performed manually by the operator. Each animal received two implants, one of each group, with randomized placement. Prior to suturing, the wound was cleaned and generously irrigated with sterile saline. The incisions through the fascia and skin were closed separately with bioresorbable sutures (Ethicon, Norderstedt, Germany). The animals were administered with post-surgical analgesic buprenorphine hydrochloride ( $0.5 \mathrm{~mL}$ Temgesic; Reckitt Benckiser, Slough, UK) for 3 days and were kept in normal cages until euthanasia. Euthanasia was performed with an overdose of sodium pentobarbital (60 mg/mL, Apoteksbolaget AB, Stockholm, Sweden). The bone-implant blocks were retrieved en bloc and immersed in $4 \%$ buffered formaldehyde for $24 \mathrm{~h}$ for fixation purpose.

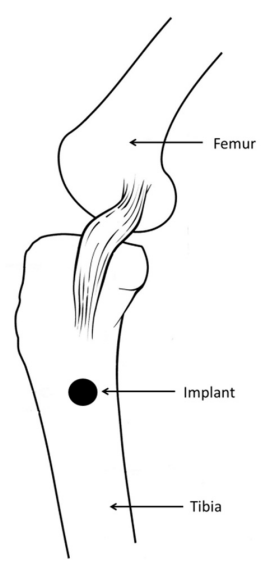

(A)

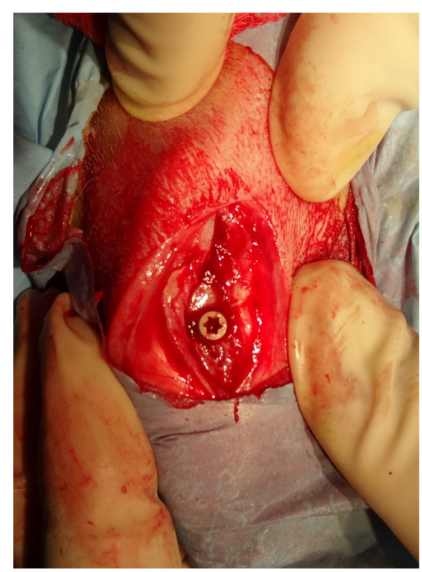

(B)

Figure 9. Illustration of implant location (A) and exposed surgical site with implant in position (B).

\subsection{Micro-CT Evaluation}

The samples were increasingly dehydrated in graded ethanol up to $99.9 \%$ (Solveco AB, Rosersberg, Sweden). Ethanol was cleared gradually and the tissue simultaneously infiltrated with resin without decalcification and finally embedded in light curing resin (Technovit 7200 VLC; Heraeus Kulzer Wehrheim, Germany).

The embedded specimens were scanned in air with a microfocus X-ray CT (MCT-CB100MF Hitachi, Medico, Tokyo, Japan). Prior to scanning, the sample was pre-scanned and placed in a custom jig and aligned in an axial direction vertical to the long axis of the implant. The sample was imaged with $201 \mu \mathrm{CT}$ slices with a single slice resolution of $51 \mu \mathrm{m}$. X-ray energy level was set to $70 \mathrm{kV}$ and a current of $100 \mu \mathrm{A}$ and scanned for $120 \mathrm{~s}$. The threshold was calculated by discriminant function analysis, around 2200 voxel value. 
The raw data from the micro-CT were reconstructed into DICOM format, imported and evaluated in a volume analysis program. (TRI 3D-BON, Ratoc system engineering, Japan) In the software the implant was vertically aligned and observed in an axial top view. To define the VOI, the implant midpoint was carefully marked out and the diameter of the implant except the threads was excluded. The slice of the implant top was selected and the length of the implant was included to VOI. The VOI was divided into three adjacent radius volumes with the same length as the implant (Figure 3 ). The inner (D1) region was selected to have the same thickness as the thread depth (64 voxels $=3.26 \mathrm{~mm}$ ) and the outer two regions (D2, D3) were set to the same thickness as D1. In order to quantify the bone morphometry, the structural properties of cortical and trabecular bone near the implant were assessed with the following parameters; bone volume to total volume ratio (BV/TV, \%), bone surface per given bone volume $(\mathrm{BS} / \mathrm{BV}, \%)$, trabecular thickness $(\mu \mathrm{m})$, trabecular number $\left(\mathrm{mm}^{-1}\right)$ and trabecular separation $(\mu \mathrm{m})$. BV/TV indicates the fraction of the selected VOI that is occupied by mineralized bone. $\mathrm{BS} / \mathrm{BV}$ reflects the bone surface on the trabecular bone in the VOI and can in bone biology provide a value of trabecular bone lining cells for potential osseointegration.

\subsection{Histomorphometry}

Embedded samples were cut and ground into sections with a thickness of approximately $20 \mu \mathrm{m}$ using a diamond blade and grinding system (Exakt, Apparatebau, Norderstedt, Germany). The sectioned samples were stained with Masson-Trichrome-Goldner stain, which has three color staining suitable for distinguishing cells and bone from connective tissue (Donath and Breuner 1982). Due to the inertness of PEEK the current staining protocol was selected with the intention to reveal non-mineralized and connective tissue in the implant-bone junction (Noiset 1999). The samples were examined under light microscopy (Eclipse ME600, Nikon, Tokyo, Japan). The percentage of bone-implant contact (BIC) and new bone area (BA) were calculated with image analysis software (Image J v. 1.43r, National Institutes of Health, Bethesda, MD, USA).

\subsection{Statistics}

Statistical analysis was performed using SPSS software (Version 20, SPSS Inc., Chicago, IL, USA). The results from the histomorphometric and micro-CT analysis were subjected to a non-parametric Wilcoxon-signed rank test (Exact sign, 2-talied). P-values $<0.05$ were considered significant.

\section{Conclusions}

This study reported a significant improvement of early bone integration for PEEK implants coated with nanosized HA. The results may be of clinical interest for early loading applications, but further studies are required to statistically verify the results and to improve the extended effect of the coating.

\section{Acknowledgments}

This study was supported by grants received from the Swedish Knowledge Foundation. 


\section{Author Contributions}

Ann Wennerberg, Per Kjellin and Ryo Jimbo conceived and designed the study; Pär Johansson and Ryo Jimbo performed the experiments; Pär Johansson, Ann Wennerberg and Ryo Jimbo analyzed the data; Per Kjellin and Fredrik Currie contributed with the implants and SEM analysis. Yusuke Kozai and Takashi Sakurai analyzed the micro-CT data. Pär Johansson wrote the majority of the paper and all authors reviewed and approved the final version.

\section{Conflicts of Interest}

The authors declare no conflict of interest.

\section{References}

1. Branemark, P.I.; Hansson, B.O.; Adell, R.; Breine, U.; Lindstrom, J.; Hallen, O.; Ohman, A. Osseointegrated implants in the treatment of the edentulous jaw. Experience from a 10-year period. Scand. J. Plast. Reconstr. Surg. Suppl. 1977, 16, 1-132.

2. Head, W.C.; Bauk, D.J.; Emerson, R.H., Jr. Titanium as the material of choice for cementless femoral components in total hip arthroplasty. Clin. Orthop. Relat. Res. 1995, 85-90.

3. Treves, C.; Martinesi, M.; Stio, M.; Gutierrez, A.; Jimenez, J.A.; Lopez, M.F. In vitro biocompatibility evaluation of surface-modified titanium alloys. J. Biomed. Mater. Res. Part A 2010, 92, 1623-1634.

4. Anandjiwala, J.; Seo, J.Y.; Ha, K.Y.; Oh, I.S.; Shin, D.C. Adjacent segment degeneration after instrumented posterolateral lumbar fusion: A prospective cohort study with a minimum five-year follow-up. Eur. Spine J. 2011, 20, 1951-1960.

5. Park, P.; Garton, H.J.; Gala, V.C.; Hoff, J.T.; McGillicuddy, J.E. Adjacent segment disease after lumbar or lumbosacral fusion: Review of the literature. Spine 2004, 29, 1938-1944.

6. Ahn, Y.H.; Chen, W.M.; Lee, K.Y.; Park, K.W.; Lee, S.J. Comparison of the load-sharing characteristics between pedicle-based dynamic and rigid rod devices. Biomed. Mater. 2008, 3, doi:10.1088/1748-6041/3/4/044101

7. Mantripragada, V.P.; Lecka-Czernik, B.; Ebraheim, N.A.; Jayasuriya, A.C. An overview of recent advances in designing orthopedic and craniofacial implants. J. Biomed. Mater. Res. Part A 2013, 101, 3349-3364.

8. Sagomonyants, K.B.; Jarman-Smith, M.L.; Devine, J.N.; Aronow, M.S.; Gronowicz, G.A. The in vitro response of human osteoblasts to polyetheretherketone (peek) substrates compared to commercially pure titanium. Biomaterials 2008, 29, 1563-1572.

9. Kurtz, S.M.; Devine, J.N. Peek biomaterials in trauma, orthopedic, and spinal implants. Biomaterials 2007, 28, 4845-4869.

10. Katzer, A.; Marquardt, H.; Westendorf, J.; Wening, J.V.; von Foerster, G. Polyetheretherketonecytotoxicity and mutagenicity in vitro. Biomaterials 2002, 23, 1749-1759.

11. Nieminen, T.; Kallela, I.; Wuolijoki, E.; Kainulainen, H.; Hiidenheimo, I.; Rantala, I. Amorphous and crystalline polyetheretherketone: Mechanical properties and tissue reactions during a 3-year follow-up. J. Biomed. Mater. Res. Part A 2008, 84, 377-383. 
12. Toth, J.M.; Wang, M.; Estes, B.T.; Scifert, J.L.; Seim, H.B., 3rd; Turner, A.S. Polyetheretherketone as a biomaterial for spinal applications. Biomaterials 2006, 27, 324-334.

13. Williams, D. Polyetheretherketone for long-term implantable devices. Med. Device Technol. 2008, 19, 10-11.

14. Rabiei, A.; Sandukas, S. Processing and evaluation of bioactive coatings on polymeric implants. J. Biomed. Mater. Res. Part A 2013, doi:10.1002/jbm.a.34557.

15. Abu Bakar, M.S.; Cheng, M.H.; Tang, S.M.; Yu, S.C.; Liao, K.; Tan, C.T.; Khor, K.A.; Cheang, P. Tensile properties, tension-tension fatigue and biological response of polyetheretherketone-hydroxyapatite composites for load-bearing orthopedic implants. Biomaterials 2003, 24, 2245-2250.

16. Converse, G.L.; Yue, W.; Roeder, R.K. Processing and tensile properties of hydroxyapatite-whisker-reinforced polyetheretherketone. Biomaterials 2007, 28, 927-935.

17. Fan, J.P.; Tsui, C.P.; Tang, C.Y.; Chow, C.L. Influence of interphase layer on the overall elasto-plastic behaviors of ha/peek biocomposite. Biomaterials 2004, 25, 5363-5373.

18. Wong, K.L.; Wong, C.T.; Liu, W.C.; Pan, H.B.; Fong, M.K.; Lam, W.M.; Cheung, W.L.; Tang, W.M.; Chiu, K.Y.; Luk, K.D.; et al. Mechanical properties and in vitro response of strontium-containing hydroxyapatite/polyetheretherketone composites. Biomaterials 2009, 30, 3810-3817.

19. Yu, S.; Hariram, K.P.; Kumar, R.; Cheang, P.; Aik, K.K. In vitro apatite formation and its growth kinetics on hydroxyapatite/polyetheretherketone biocomposites. Biomaterials 2005, 26, 2343-2352.

20. Peraire, C.; Arias, J.L.; Bernal, D.; Pou, J.; Leon, B.; Arano, A.; Roth, W. Biological stability and osteoconductivity in rabbit tibia of pulsed laser deposited hydroxylapatite coatings. J. Biomed. Mater. Res. Part A 2006, 77, 370-379.

21. Barkarmo, S.; Wennerberg, A.; Hoffman, M.; Kjellin, P.; Breding, K.; Handa, P.; Stenport, V. Nano-hydroxyapatite-coated peek implants: A pilot study in rabbit bone. J. Biomed. Mater. Res. Part A 2013, 101, 465-471.

22. Meirelles, L.; Arvidsson, A.; Andersson, M.; Kjellin, P.; Albrektsson, T.; Wennerberg, A. Nano hydroxyapatite structures influence early bone formation. J. Biomed. Mater. Res. Part A 2008, 87, 299-307.

23. Weiner, B.K.; Fraser, R.D. Spine update lumbar interbody cages. Spine 1998, 23, 634-640.

24. Abrahamsson, I.; Linder, E.; Larsson, L.; Berglundh, T. Deposition of nanometer scaled calcium-phosphate crystals to implants with a dual acid-etched surface does not improve early tissue integration. Clin. Oral Implants Res. 2013, 24, 57-62.

25. Fontana, F.; Rocchietta, I.; Addis, A.; Schupbach, P.; Zanotti, G.; Simion, M. Effects of a calcium phosphate coating on the osseointegration of endosseous implants in a rabbit model. Clin. Oral Implants Res. 2011, 22, 760-766.

26. Gobbato, L.; Arguello, E.; Martin, I.S.; Hawley, C.E.; Griffin, T.J. Early bone healing around 2 different experimental, ha grit-blasted, and dual acid-etched titanium implant surfaces. A pilot study in rabbits. Implant Dent. 2012, 21, 454-460.

27. Johansson, P. Biomechanical evaluation and surface characterization of a nano modified surface on peek implants: A study in the rabbit tibia. Int. J. Nanomed. 2014, doi:10.2147/IJN.S60387. 
28. Eom, T.G.; Jeon, G.R.; Jeong, C.M.; Kim, Y.K.; Kim, S.G.; Cho, I.H.; Cho, Y.S.; Oh, J.S. Experimental study of bone response to hydroxyapatite coating implants: Bone-implant contact and removal torque test. Oral Surg. Oral Med. Oral Pathol. Oral Radiol. 2012, 114, 411-418.

29. Jimbo, R.; Xue, Y.; Hayashi, M.; Schwartz-Filho, H.O.; Andersson, M.; Mustafa, K.; Wennerberg, A. Genetic responses to nanostructured calcium-phosphate-coated implants. J. Dent. Res. 2011, 90, $1422-1427$.

30. Reigstad, O.; Johansson, C.; Stenport, V.; Wennerberg, A.; Reigstad, A.; Rokkum, M. Different patterns of bone fixation with hydroxyapatite and resorbable cap coatings in the rabbit tibia at 6 , 12, and 52 weeks. J. Biomed. Mater. Res. Part B Appl. Biomater. 2011, 99, 14-20.

31. Lee, J.H. Quantitative comparison of novel cao-sio2-p2o5-b2o3 glass-ceramics (bgs-7) with hydroxyapatite as bone graft extender in rabbit ilium. Tissue Eng. Regen. Med. 2010, 7, 540-547.

32. Lee, J.H.; Ryu, H.S.; Lee, D.S.; Hong, K.S.; Chang, B.S.; Lee, C.K. Biomechanical and histomorphometric study on the bone-screw interface of bioactive ceramic-coated titanium screws. Biomaterials 2005, 26, 3249-3257.

33. Kim, D.S.; Kim, D.G.; Park, C.J.; Cho, L.R. Histomorphometry and stability analysis of early loaded implants with two different surface conditions in beagle dogs. J. Adv. Prosthodont. 2009, $1,10-18$.

34. Wennerberg, A.; Albrektsson, T.; Andersson, B.; Krol, J.J. A histomorphometric and removal torque study of screw-shaped titanium implants with three different surface topographies. Clin. Oral Implants Res. 1995, 6, 24-30.

35. Kim, M.C.; Chung, H.T.; Cho, J.L.; Kim, D.J.; Chung, N.S. Subsidence of polyetheretherketone cage after minimally invasive transforaminal lumbar interbody fusion. J. Spinal Disord. Tech. 2013, 26, 87-92.

36. Albrektsson, T.; Wennerberg, A. Oral implant surfaces: Part 1-Review focusing on topographic and chemical properties of different surfaces and in vivo responses to them. Int. J. Prosthodont. 2004, 17, 536-543.

37. Meirelles, L.; Currie, F.; Jacobsson, M.; Albrektsson, T.; Wennerberg, A. The effect of chemical and nanotopographical modifications on the early stages of osseointegration. Int. J. Oral Maxillofac. Implants 2008, 23, 641-647.

38. Soballe, K.; Overgaard, S.; Hansen, E.S.; Brokstedt-Rasmussen, H.; Lind, M.; Bunger, C. A review of ceramic coatings for implant fixation. J. Long-Term Effects Med. Implants 1999, 9, 131-151.

39. Stein, I.C.; Than, K.D.; Chen, K.S.; Wang, A.C.; Park, P. Failure of a polyether-ether-ketone expandable interbody cage following transforaminal lumbar interbody fusion. Eur. Spine J. 2014, $24,555-559$.

40. Kim, P.D.; Baron, E.M.; Levesque, M. Extrusion of expandable stacked interbody device for lumbar fusion: Case report of a complication. Spine 2012, 37, E1155-E1158.

41. Chou, W.K.; Chien, A.; Wang, J.L. Biomechanical analysis between peek and titanium screw-rods spinal construct subjected to fatigue loading. J. Spinal Disord. Tech. 2014, 28, E121-E125.

42. Rao, P.J.; Pelletier, M.H.; Walsh, W.R.; Mobbs, R.J. Spine interbody implants: Material selection and modification, functionalization and bioactivation of surfaces to improve osseointegration. Orthop. Surg. 2014, 6, 81-89. 
43. Nemoto, O.; Asazuma, T.; Yato, Y.; Imabayashi, H.; Yasuoka, H.; Fujikawa, A. Comparison of fusion rates following transforaminal lumbar interbody fusion using polyetheretherketone cages or titanium cages with transpedicular instrumentation. Eur. Spine J. 2014, 23, 2150-2155.

44. Kruger, T.; Alter, C.; Reichel, H.; Birke, A.; Hein, W.; Spielmann, R.P. Possibilities of follow-up imaging after implantation of a carbon fiber-reinforced hip prosthesis. Aktuelle Radiol. 1998, 8, 81-86.

45. Numata, Y.; Sakae, T.; Nakada, H.; Suwa, T.; LeGeros, R.Z.; Okazaki, Y.; Kobayashi, K. Micro-CT analysis of rabbit cancellous bone around implants. J. Hard Tissue Biol. 2007, 16, 91-93.

46. Voor, M.J.; Yang, S.; Burden, R.L.; Waddell, S.W. In vivo micro-ct scanning of a rabbit distal femur: Repeatability and reproducibility. J. Biomech. 2008, 41, 186-193.

47. Kjellin, P.; Andersson, M. Synthetic Nano-Sized Crystalline Calcium Phosphate and Method of Production. US Pantent 8206813 B2, 26 June 2012.

(C) 2015 by the authors; licensee MDPI, Basel, Switzerland. This article is an open access article distributed under the terms and conditions of the Creative Commons Attribution license (http://creativecommons.org/licenses/by/4.0/). 
III 



\section{Polyether ether ketone implants achieve increased bone fusion when coated with nano-sized hydroxyapatite: a histomorphometric study in rabbit bone}

Pär Johansson'

Ryo Jimbo'

Yoshihito Naito ${ }^{2}$

Per Kjellin ${ }^{3}$

Fredrik Currie ${ }^{3}$

Ann Wennerberg'

'Department of Prosthodontics, Faculty of Odontology, Malmö

University, Malmö, Sweden; ${ }^{2}$ Oral Implant Center, Tokushima University Hospital, Tokushima, Japan; ${ }^{3}$ Promimic $A B$, Stena Center, Göteborg, Sweden
Correspondence: Pär Johansson Department of Prosthodontics, Faculty of Odontology, Malmö University, Car Gustafs väg 34, 21421 Malmö, Sweden $\mathrm{Tel}+46406658514$

Email par.johansson@mah.se

\begin{abstract}
Polyether ether ketone (PEEK) possesses excellent mechanical properties similar to those of human bone and is considered the best alternative material other than titanium for orthopedic spine and trauma implants. However, the deficient osteogenic properties and the bioinertness of PEEK limit its fields of application. The aim of this study was to limit these drawbacks by coating the surface of PEEK with nano-scaled hydroxyapatite (HA) minerals. In the study, the biological response to PEEK, with and without HA coating, was investigated. Twenty-four screw-like and apically perforated implants in the rabbit femur were histologically evaluated at 3 weeks and 12 weeks after surgery. Twelve of the 24 implants were HA coated (test), and the remaining 12 served as uncoated PEEK controls. At 3 weeks and 12 weeks, the mean bone-implant contact was higher for test compared to control $(P<0.05)$. The bone area inside the threads was comparable in the two groups, but the perforating hole showed more bone area for the HA-coated implants at both healing points $(P<0.01)$. With these results, we conclude that nano-sized HA coating on PEEK implants significantly improved the osteogenic properties, and in a clinical situation this material composition may serve as an implant where a rapid bone fusion is essential.
\end{abstract}

Keywords: HA, PEEK, osseointegration, histology, orthopedics, in vivo

\section{Introduction}

Few new materials are introduced to the medical device industry, partly because of the good quality of the existing ones and the extremely high cost for introducing new ones. Despite this, polyether ether ketone (PEEK) was introduced where metallic implants such as $\mathrm{Ti}$ alloys and $\mathrm{CoCr}$ alloys experienced inferior outcomes due to their mechanical properties. ${ }^{1-5}$ One such potential shortcoming of conventional implant materials is the tenfold higher elastic modulus of titanium compared to bone that has been found to cause degeneration of adjacent segments via stress shielding. To overcome these problems, PEEK was introduced into the orthopedic field as an implant material due to its similar elastic modulus as that of human cortical bone. ${ }^{6}$ For the past 2 decades, PEEK has become a common alternative to metal implants in several orthopedic applications. ${ }^{7,8}$

However, PEEK has shown both in vivo and in vitro studies to have low bioactive features due to the relatively bioinert surface..$^{9-11}$ Therefore, many studies have been conducted to improve the biocompatible state of PEEK by incorporating bioactive substances into the substrate or onto the surface as a coating. ${ }^{12-14}$ The authors have 
found that incorporation of hydroxyapatite (HA) into the PEEK compound substantially decreased the mechanical properties of the material. ${ }^{14,15}$ Instead, by altering the surface topography and chemical composition, the surface becomes more bioactive and an improved bone formation can be achieved owing to an increased surface area and higher surface energy. ${ }^{3}$ Therefore, several techniques to coat the PEEK surface have been evaluated. Plasma sprayed deposition and electron beam deposition are two common methods to coat the surface with HA, which improved the bioactive potential of PEEK. However, the long-term effect of these techniques on titanium has been questioned after retrieved sections have demonstrated inflammation cells adjacent to detached coating fragments. The coating thickness and mechanical attachment to the substrate were hypothesized to be one reason to cause this incident. ${ }^{16-18}$

In this study, a liquid-based nano-sized HA solution was applied to a PEEK surface with a wet chemically based technique, and the excess was removed with pressurized gas and by spinning the implant. The final step in this coating method is a brief heat treatment, which is performed at a temperature just below the melting point of the PEEK polymer. This will create a sintering effect that adheres the crystals to the PEEK substrate. The end result is an extremely thin layer of ceramic coating where the risk of delamination becomes minimal. In other studies, this exceptional technique has been found to successfully increase the bone formation on titanium but it has never been applied on PEEK. ${ }^{9-21}$ Using HA in the form of nanoparticles increases not only the surface area but also the interaction and adhesion between the crystals and the PEEK surface. Another unique feature of this study was a perforating hole of the implant that permits evaluation of the osteoconductive effect of the surface modification. Osteoconductive ability is a decisive feature when bone fusion is requested, for instance, in several spinal applications.

The aim of this study was to evaluate the bone formation around and bone conductivity into the apical hole of the PEEK implant (Figure 1). One group was coated with nanocrystalline HA, and the comparison was made against an untreated PEEK implant. The experiment was carried out in a well-documented rabbit femur model.

\section{Materials and methods Implant surface preparation}

Forty-eight noncutting PEEK implants (Invibio Ltd, Lancashire, UK) with a diameter of $3.5 \mathrm{~mm}$ and a length of $4 \mathrm{~mm}$ were prepared for the study. The implants were machined with a hole penetrating the apical parts to provide

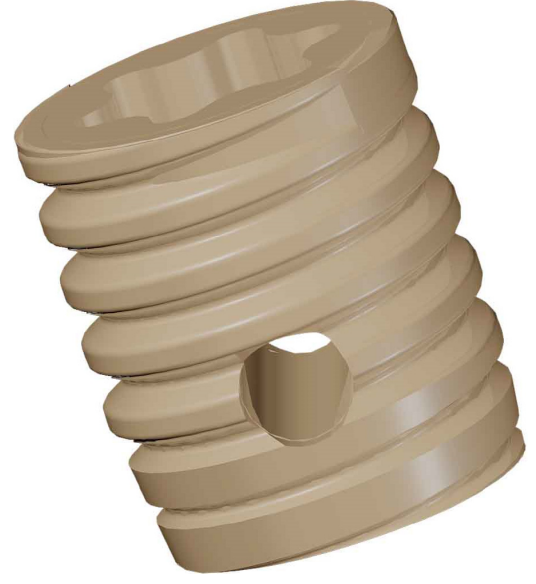

Figure I A 3D rendered image of the PEEK implant. Abbreviations: 3D, three-dimensional; PEEK, polyether ether ketone.

a fully intact coated surface, even after insertion, which allow evaluation of the bone ingrowth (Figure 1). This unique design permits evaluation of the surface osteoconductivity where the amount of newly formed bone within the hole can be measured. The surface of half the total amount of implants $(n=24)$ was coated with the HA ${ }^{\text {nano }}$ Surface provided by Promimic AB, Göteborg, Sweden (test), whereas the remaining implants $(n=24)$ were used with their native surfaces (control). For the implants to be coated, $50 \mu \mathrm{L}$ of coating solution was applied on top of each implant. The implant was then rotated at 2,700 rpm for 5 seconds, in order to create a homogenous layer of coating liquid onto the implant surface. The coating solution was a microemulsion, containing nano-sized HA crystals, 20-50 nm long and 2-10 nm wide, dispersed with water, surfactants, and organic solvent. The coating solution is more thoroughly described in a patent description. ${ }^{22}$ After the coating procedure, the PEEK implant was put into an oven having an oxygen-enriched atmosphere at $325^{\circ} \mathrm{C}$ for 5 minutes. This heat treatment was done to remove the surfactants and to adhere the crystals onto the PEEK surface. The thickness of the resulting HA layer was $20-40 \mathrm{~nm}$.

\section{Morphological characterization}

The surface morphology of the study's assigned PEEK implants was performed with a scanning electron microscopy (LEO Ultra 55 FEG; ZEISS, Oberkochen, Germany) 
at an accelerating voltage of $2-4 \mathrm{kV}$. To make the surface conductive, the implants were sputtered with gold, using a JEOL JFC-1100E (JEOL Ltd, Tokyo, Japan) operating at $10 \mathrm{kV}$ for 90 seconds. The nano and micro topographies of the implants are described in a previous report. ${ }^{23}$

\section{Surgical procedure and implantation}

The animal study was approved by the Malmö/Lund (Sweden) regional animal ethics committee and all experiments were performed following the institutional and national guidelines and regulations of the Board of Agriculture. Twenty-four Swedish lop-eared rabbits (mean weight $4.1 \mathrm{~kg}$ ) received one HA and one control implant each, which were randomly inserted into the cancellous bone of the left and right distal femur. The rabbits were divided into two groups $(n=12)$ for observation periods of either 3 weeks or 12 weeks after implant surgery. The healing times, 3 weeks and 12 weeks, refer to the early stage of bone formation and a complete bone healing, respectively. An anesthetic dose of $0.15 \mathrm{~mL} / \mathrm{kg}$ medetomidine ( $1 \mathrm{mg} / \mathrm{mL}$ Domitor; Orion Pharma, Sollentuna, Sweden) and $0.35 \mathrm{~mL} / \mathrm{kg}$ ketamine hydrochloride (50 mg/mL Ketalar; Pfizer Inc., New York, NY, USA) was administered. The implant site was disinfected ( $70 \%$ ethanol; Solveco AB, Rosersberg, Sweden, and $5 \mathrm{mg} / \mathrm{mL}$ chlorhexidine; Fresenius Kabi AB, Uppsala, Sweden), and additional local anesthesia (lidocaine hydrochloride, Xylocaine; AstraZeneca plc, Södertälje, Sweden) was injected subcutaneously before a skin incision was made to expose the bone. The bone was trephined with a series of drills up to a final diameter of $3.2 \mathrm{~mm}$. Tapping and insertion was performed manually before the implant was installed within the femoral condyle (Figure 2A and B). This implant location provides bone with trabecular structure that covers the entire implant surface. The wound was cleaned and generously irrigated with sterile saline before the fascia and skin was closed separately with bioresorbable sutures (Ethicon, Norderstedt, Germany). Postsurgical analgesic buprenorphine hydrochloride $(0.5 \mathrm{~mL}$ Temgesic; Reckitt Benckiser, Slough, UK) was administrated the next 3 days. The animals were euthanized with an overdose of sodium pentobarbital $(60 \mathrm{mg} / \mathrm{mL}$; Apoteksbolaget $\mathrm{AB}$, Stockholm, Sweden). Bone-implant blocks (Figure 2A and $B$ ) were retrieved en bloc, immersed, and fixated in $4 \%$ buffered formaldehyde for 24 hours.

\section{Histomorphometry}

The bone-implant blocks were increasingly dehydrated in graded ethanol up to $99.9 \%$ (Solveco AB). Gradually, the ethanol was cleared and the tissue was simultaneously infiltrated with resin without decalcification. Finally, the specimens were embedded in light-curing resin (Technovit 7200 VLC; Heraeus Kulzer, Wehrheim, Germany).

The samples were cut and ground using a diamond blade and grinding system (Exakt; Apparatebau, Norderstedt, Germany) into a final thickness of $\sim 20 \mu \mathrm{m}$ and thereafter stained with toluidine blue. The cut was aligned along the apical hole in order to evaluate bone area (BA), bone-implant contact (BIC), and BA inside the hole on the same histological section. The evaluation was carried out under a light microscopy (Eclipse ME600, Nikon Corporation, Tokyo, Japan). The percentage of BIC and new BA in the threads
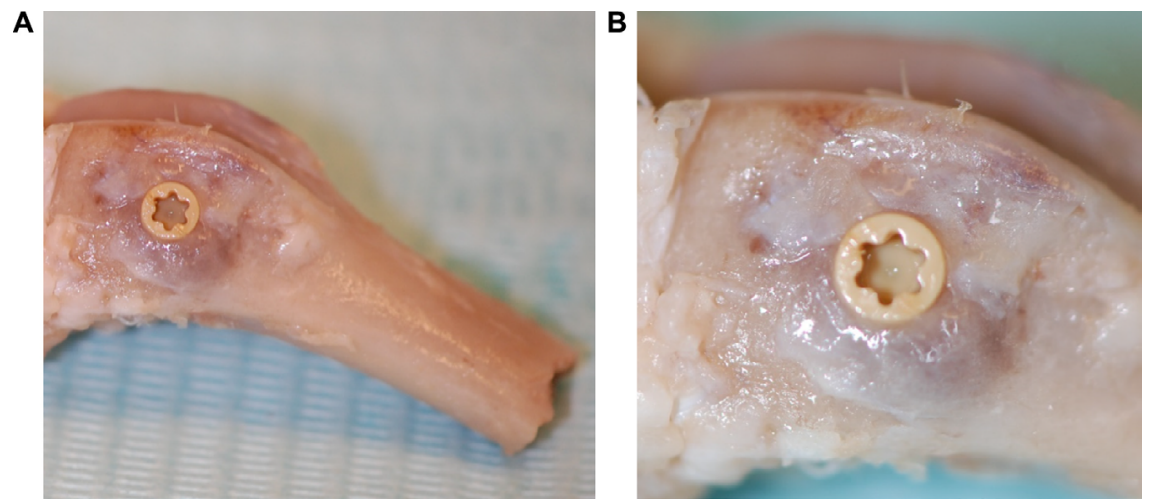

Figure 2 Clinical images of bone block (A) after retrieval demonstrating implant positioning and (B) with a magnified image of the implant in situ. 


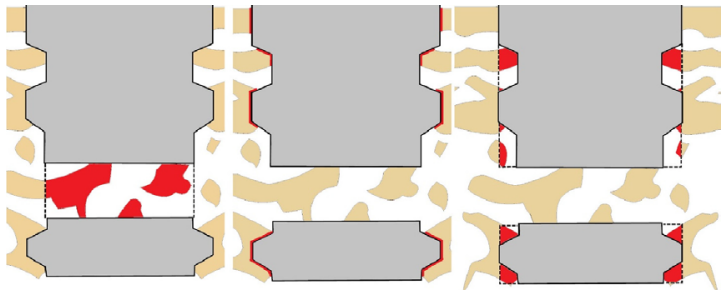

Figure 3 A schematic image of the area of interest in the quantitative histomorphometric analysis.

Notes: The measured area of interest is colored red and bone is colored tan. Bone area inside the perforating hole (left), bone-implant contact (middle), and bone area inside threads (right)

and inside the hole were calculated with image analysis software (ImageJ Version 1.43r; National Institutes of Health, Bethesda, MD, USA). Figure 3 shows a schematic illustration of the histomorphometric measurement areas.

\section{Statistics}

The histological data were analyzed with a nonparametric Mann-Whitney test with a level of significance set at 0.05 . The analysis was performed using SPSS software (Version 20; IBM Corporation, Armonk, NY, USA).

\section{Results}

\section{Morphological and topographical analyses}

Scanning electron micrograph images of the two types of implant surfaces and their typical surface features are given in Figure 4A and B, for two magnifications. At a higher magnification $(80 \mathrm{~K})$ of the coated surface, the HA minerals appear as rod-shaped particles fully covering the PEEK surface (Figure 4B). The rods are arranged in different directions with a size of $\sim 10 \mathrm{~nm}$ width and $150 \mathrm{~nm}$ length. At a lower resolution (40K), there were no remarkable topographical differences between the two surfaces, with the possible exception that the HA particles have leveled out the striations from the turning procedure (Figure 4A).

\section{Histomorphometry}

At the time of implant retrieval, there were no clinical signs of infection and all the implants were immobilized without any visible signs of marginal bone resorption. The qualitative histological evaluation revealed stained woven bone close to the implant surface and deeper stained lamellar bone in proximity to the implants (Figure 5A and D). A Haversian system can be seen within the thread of an HA-coated surface after 12 weeks of healing (Figure 5C). No immunological cells were seen in proximity to the implanted materials. In the perforated hole at 3 weeks, the bone had extended $>50 \%$ of the diameter of the hole with irregular contact to the inner wall of the implant (Figure 5A and B).

The quantitative evaluation of the BIC revealed newly formed bone in contact with the implant surface. The BIC after 3 weeks was significantly higher for the test implants with a value of $14.1 \% \pm 3.5 \%$ compared to $11.1 \% \pm 3.5 \%$ for
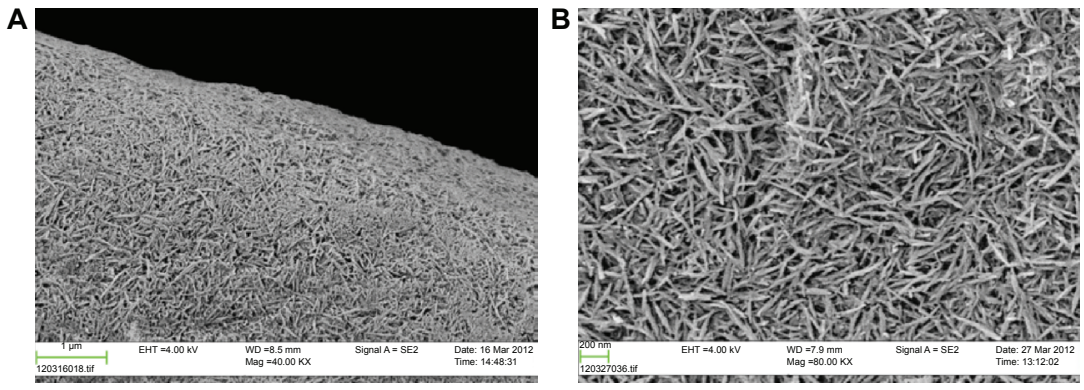

Figure 4 SEM photographs of hydroxyapatite-coated PEEK in two magnifications: 40K (A) and 80K (B). Abbreviations: SEM, scanning electron microscopy; PEEK, polyether ether ketone. 

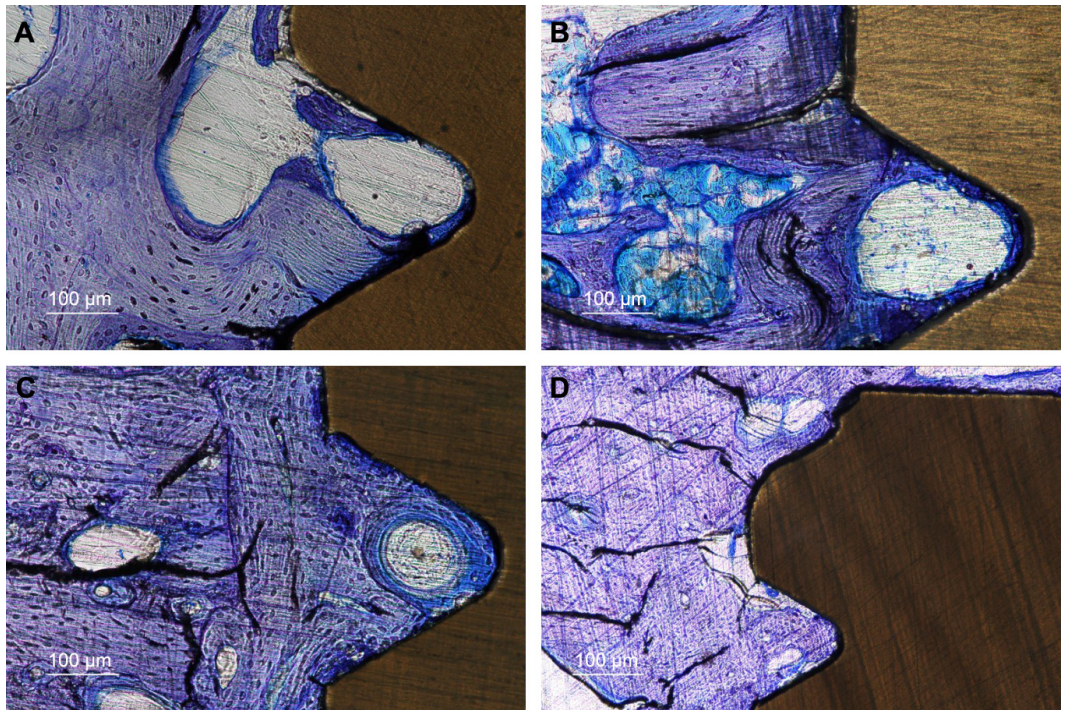

Figure 5 Ground sections $\times 10$ of HA-coated and pure PEEK implants.

Notes: Three weeks after insertion, the HA-coated PEEK (A) shows more osteocytes and lamellar bone compared to the pure PEEK (B). After 12 weeks of healing, the HA-coated PEEK (C) shows signs of vital and rapid bone formation with a Haversian system inside the thread surrounded by large amounts of osteocytes. The uncoated PEEK implant after 12 weeks also demonstrates large amounts of osteocytes but with a lower extent of bone-implant contact (D).

Abbreviations: HA, hydroxyapatite; PEEK, polyether ether ketone.

the control. After 12 weeks, the BIC was also found to be significantly higher for the test group $(P=0.038$; Table 1 and Figure 6A-C). Comparing the BA in the threads of the two groups revealed no significant difference at any healing time point (3 weeks: $P=0.478,12$ weeks: $P=0.211$ ). However, the BA in the apical hole demonstrated a significant difference for the test group ( 3 weeks: $P=0.00,12$ weeks: $P=0.01$ ). At 3 weeks healing, the mean $\mathrm{BA}$ in the hole for the test and control was $17.2 \% \pm 7.8 \%$ and $4.52 \% \pm 2.9 \%$, respectively. The BA after 12 weeks demonstrated a significant difference

Table I Summary of the histomorphometric measurements

\begin{tabular}{clll}
\hline & BIC (\%, SD) & BA-thread (\%, SD) & BA-hole (\%, SD) \\
\hline 3 weeks & & & \\
Control & $11.08(3.49)$ & $25.04(7.64)$ & $4.52(2.9)$ \\
Test & $14.07(3.54)$ & $27.68(8.31)$ & $17.21(7.75)$ \\
$P$-value & $\mathbf{0 . 0 3 4}$ & 0.478 & $\mathbf{0 . 0 0 0}$ \\
I2 weeks & & & \\
Control & $11.39(3.8 I)$ & $44.48(10.78)$ & $10.80(5.53)$ \\
Test & $16.65(6.74)$ & $49.66(13.56)$ & $21.33(6.94)$ \\
$P$-value & $\mathbf{0 . 0 3 8}$ & 0.211 & $\mathbf{0 . 0 0 1}$ \\
\hline
\end{tabular}

Note: Bold $P$-values show significant values.

Abbreviations: BIC, bone-implant contact; BA, bone area; SD, standard deviation. with mean values for test implants of $21.3 \% \pm 6.9 \%$ and for control implants of $10.8 \% \pm 5.5 \%$. Figure $7 \mathrm{~A}$ shows a coated PEEK implant after 3 weeks of healing where the bone has managed to migrate through the entire hole. In Figure 7B, the line between old and new bones is clear to distinguish and the bone extends though the hole while making contact with the implant surface at regular intervals.

Figure 7A shows a coated PEEK implant after 3 weeks of healing where the bone has managed to migrate through the entire hole. In Figure 7B, the line between old and new bones is clear to distinguish and the bone extends through the hole while making contact with the implant surface at regular intervals.

\section{Discussion}

The aim of this experimental study was to evaluate the effect of a nano-sized HA coating on bone formation of PEEK implants after 3 and 12 weeks of healing in a rabbit femur model. We found that osseointegration of HA-coated PEEK was higher compared with uncoated PEEK implants. HA coating on PEEK significantly improved the BIC at both 

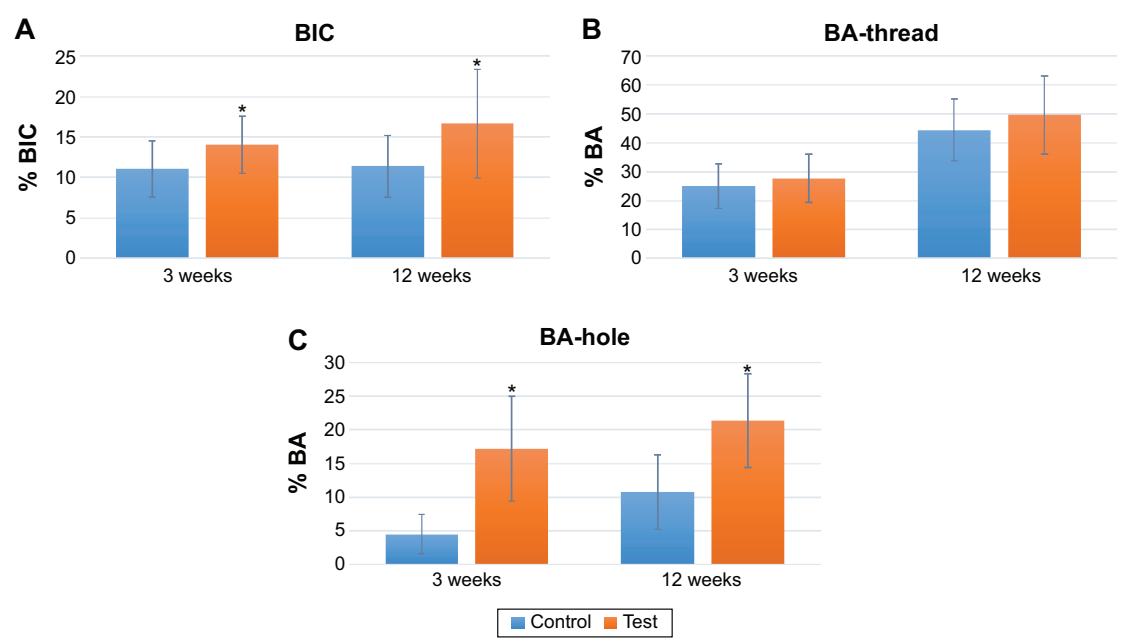

Figure 6 Graphs presenting the bone-implant contact (A), bone area (B), and bone area inside the perforating hole (C) at 3 weeks and 12 weeks of healing. Note: *Represents a statistical significance with a $P$-value $<0.05$.

Abbreviations: BIC, bone-implant contact; $\mathrm{BA}$, bone area.
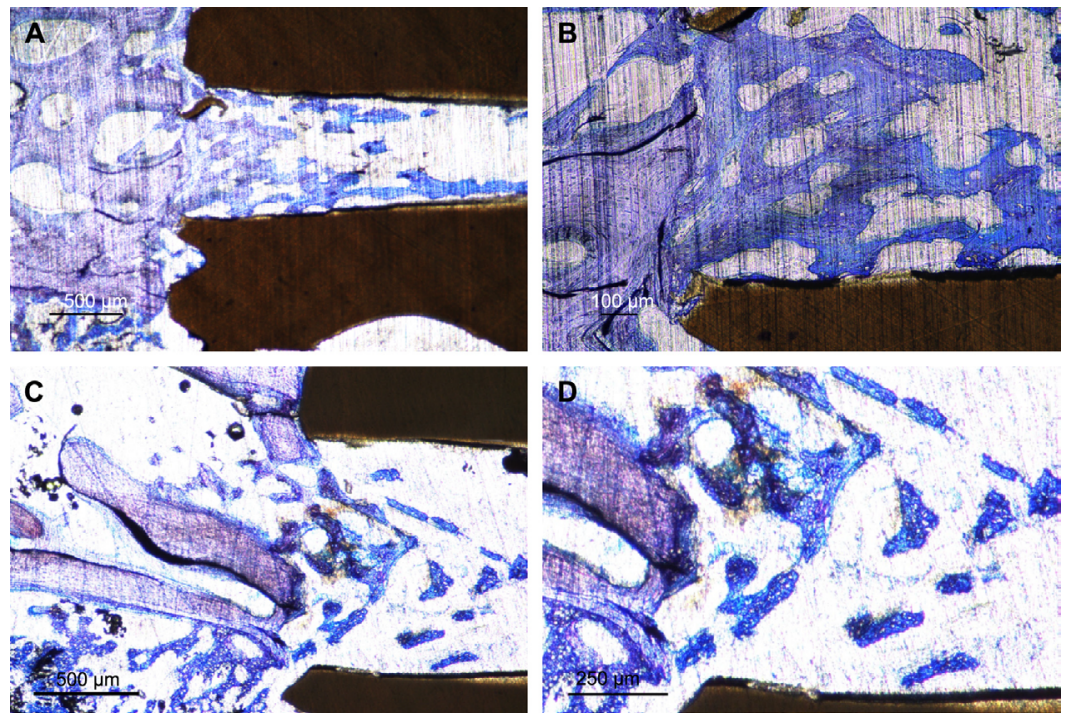

Figure 7 Histological sections in two magnifications showing the apical perforation of HA-coated (A and B) and uncoated (C and D) PEEK implants, 3 weeks after insertion.

Notes: The HA-coated implant in lower magnification (A) shows bone growth through the entire hole. In the enlarged image (B), the line between the new bone and old bone can be tracked, and inside the hole, the bone approaches the implant surface with regular intervals through the hole. The uncoated PEEK implant demonstrates significantly less bone area at the entrance and inside the perforated hole compared to that of HA-coated PEEK (C). With the higher magnification, the bone structure was found to be located in the center of the perforation without having contact to the implant surface (D).

Abbreviations: HA, hydroxyapatite; PEEK, polyether ether ketone. 
healing times. However, the BA in the threads did not show any differences between the two groups at any healing point. Moreover, the perforating hole in the apical part of the implant revealed a clear and significant increased BA for the HAcoated PEEK implants. There are several possible explanations for these findings. One major difference was the direct contact with native bone for the threads after insertion, while the hole was only filled with blood without any initial bone contact. The regenerative process around mature bone next to the threads is different from the process inside the hole, which may affect the coating abilities. Furthermore, some of the coating may have been scraped off during insertion, despite failure to observe this in a previously conducted in vitro investigation. ${ }^{24}$ These results are consistent with the findings from several other studies that evaluated HA coating on various implant materials. ${ }^{8,25}$ Sandén et al evaluated a traditional HA coating on stainless steel in a goat model and found improved bone formation compared with untreated PEEK after 6 and 12 weeks of healing. ${ }^{26}$ However, their coating was deposited with a plasma spraying technique that provides a thicker coating without nanostructures. Similar results were published by $\mathrm{Gu}$ et al who demonstrated greater bone fusion for HA-coated intervertebral cages. ${ }^{27}$ Suska et al compared HA-coated PEEK and titanium with uncoated PEEK in a rabbit model and demonstrated that HA coating significantly enhances the bone response. Moreover, in the cortical bone (tibia) model, HA-coated PEEK showed significantly higher bone values than those found with HA-coated titanium implants. ${ }^{28}$ Nakahara et al revealed improved BIC for HA-coated PEEK after 6 weeks and 12 weeks of unloaded healing. The authors indicate a correlation between BIC and shear strength, both of which were enhanced for HA-coated implants compared to noncoated implants. ${ }^{29}$ Our research team found a similar correlation as Nakahara et al with our previous study using an identical implant design. The increased removal torque for the HA-coated implants compared to uncoated controls is correlated to the higher BIC for test implants. ${ }^{23}$

Another previous study by our team evaluated the same implant design and surface modification inserted in the rabbit tibia. The results revealed a significantly higher BIC and BA after 3 weeks for HA-coated implants. ${ }^{24}$ However, after 12 weeks, the BIC in the 12-week group did not show any differences between the groups, but the mean BA values were still significantly higher on the HA-coated implants. The gathered results from the previous and the present studies indicate a pronounced effect of the HA at the early stages, even for the part of the implant inside the bone marrow where less BIC would be expected. In the femur, on the other hand, the entire implant surface was in contact with the bone resulting in a higher percent of BIC and BA compared to the tibia. The higher BIC in the femur may be due to its higher ductile properties compared to those of tibia, which minimize compression and stress around the implant. The HA-coated implants in tibia and femur show at the early stage (3 weeks) a clear advantage on the BIC, but after the longer evaluation time ( 12 weeks), the percent of contact has almost ceased in progression when compared to uncoated implants. However, the amount of BA in the thread for both tibia and femur has almost been doubled from 3 to 12 weeks and still shows significance for the HA-coated implants.

PEEK is today used in several orthopedic therapies, including spinal fusion where the implant replaces the disc and allows a fusion of bone between the vertebras. Therefore, in this study, the implant was designed with a hole, perforating the apical part of the implant that allows evaluation of bone fusion and the coated surface's ability to induce osseointegration. As far as we know, no studies have previously been published with similar implant design and experimental model. However, some studies were performed with PEEK in different spine models of animals or humans. Pelletier et al compared uncoated PEEK and titanium in a sheep spine model and found that the bone fusion was similar for both groups. The authors concluded that PEEK may be a suitable replacement for titanium in spinal fusion procedures. ${ }^{30}$ Another study by Sinclair et al evaluated uncoated PEEK and porous tantalum in a cervical interbody fusion model in goat and found higher rate of fusion for tantalum. ${ }^{31}$

\section{Conclusion}

This study demonstrated that nano-sized HA coating on PEEK implants in rabbit femur significantly improved the BIC and demonstrated strong osseoconductive properties inside the perforated hole. In a clinical situation, this material composition may improve the outcomes of different spinal procedures where a rapid bone fusion is essential.

\section{Acknowledgment}

This study was supported by grants achieved from the Swedish Knowledge Foundation and King Gustaf V's and Queen Victoria's Freemason Foundation.

\section{Disclosure}

The authors report no conflicts of interest in this work.

\section{References}

1. Park P, Garton HJ, Gala VC, Hoff JT, McGillicuddy JE. Adjacent segment disease after lumbar or lumbosacral fusion: review of the literature. Spine. 2004;29(17):1938-1944. 
2. Anandjiwala J, Seo JY, Ha KY, Oh IS, Shin DC. Adjacent segment degeneration after instrumented posterolateral lumbar fusion: a prospective cohort study with a minimum five-year follow-up. Eur Spine J. 2011;20(11):1951-1960.

3. Ha SW, Kirch M, Birchler F, et al. Surface activation of polyetheretherketone (PEEK) and formation of calcium phosphate coatings by precipitation. J Mater Sci Mater Med. 1997;8(11):683-690.

4. Kurtz SM, Devine JN. PEEK biomaterials in trauma, orthopedic, and spinal implants. Biomaterials. 2007;28(32):4845-4869.

5. Chou WK, Chien A, Wang JL. Biomechanical analysis between PEEK and titanium screw-rods spinal construct subjected to fatigue loading. J Spinal Disord Tech. 2015;28(3):E121-E125.

6. Steinberg EL, Rath E, Shlaifer A, Chechik O, Maman E, Salai M. Carbon fiber reinforced PEEK Optima - a composite material biomechanical properties and wear/debris characteristics of CF-PEEK composites for orthopedic trauma implants. J Mech Behav Biomed Mater. 2013;17:221-228.

7. Mohammadi S, Esposito M, Hall J, Emanuelsson L, Krozer A, Thomsen P. Short-term bone response to titanium implants coated with thin radiofrequent magnetron-sputtered hydroxyapatite in rabbits. Clin Implant Dent Relat Res. 2003;5(4):241-253.

8. Barkarmo S, Andersson M, Currie F, et al. Enhanced bone healing around nanohydroxyapatite-coated polyetheretherketone implants: an experimental study in rabbit bone. J Biomater Appl. 2014;29(5):737-747.

9. Katzer A, Marquardt H, Westendorf J, Wening JV, von Foerster G. Polyetheretherketone - cytotoxicity and mutagenicity in vitro. Biomaterials. 2002;23(8):1749-1759.

10. Morrison C, Macnair R, MacDonald C, Wykman A, Goldie I, Grant MH. In vitro biocompatibility testing of polymers for orthopaedic implants using cultured fibroblasts and osteoblasts. Biomaterials. 1995;16(13): 987-992.

11. Rivard $\mathrm{CH}$, Rhalmi S, Coillard C. In vivo biocompatibility testing of peek polymer for a spinal implant system: a study in rabbits. $J$ Biomed Mater Res. 2002;62(4):488-498.

12. Deligianni DD, Katsala ND, Koutsoukos PG, Missirlis YF. Effect of surface roughness of hydroxyapatite on human bone marrow cell adhesion, proliferation, differentiation and detachment strength. Biomaterials. 2001;22(1):87-96.

13. Converse GL, Yue W, Roeder RK. Processing and tensile properties of hydroxyapatite-whisker-reinforced polyetheretherketone. Biomaterials. 2007;28(6):927-935.

14. Wong KL, Wong CT, Liu WC, et al. Mechanical properties and in vitro response of strontium-containing hydroxyapatite/polyetheretherketone composites. Biomaterials. 2009;30(23-24):3810-3817.

15. Abu Bakar MS, Cheng MH, Tang SM, et al. Tensile properties, tension-tension fatigue and biological response of polyetheretherketonehydroxyapatite composites for load-bearing orthopedic implants. Biomaterials. 2003;24(13):2245-2250.

16. Cheang P, Khor KA. Addressing processing problems associated with plasma spraying of hydroxyapatite coatings. Biomaterials. 1996; 17(5):537-544.
17. Rokkum M, Reigstad A, Johansson CB. HA particles can be released from well-fixed HA-coated stems: histopathology of biopsies from 20 hips 2-8 years after implantation. Acta Orthop Scand. 2002; 73(3):298-306.

18. Albrektsson T. Hydroxyapatite-coated implants: a case against their use. J Oral Maxillofac Surg. 1998;56(11):1312-1326.

19. Meirelles L, Arvidsson A, Andersson M, Kjellin P, Albrektsson T, Wennerberg A. Nano hydroxyapatite structures influence early bone formation. J Biomed Mater Res A. 2008;87(2):299-307.

20. Svanborg LM, Hoffman M, Andersson M, Currie F, Kjellin P, Wennerberg A. The effect of hydroxyapatite nanocrystals on early bone formation surrounding dental implants. Int J Oral Maxillofac Surg. 2011;40(3):308-315.

21. Jimbo R, Xue Y, Hayashi M, et al. Genetic responses to nanostructured calcium-phosphate-coated implants. J Dent Res. 2011;90(12): 1422-1427.

22. Kjellin P, Andersson M, inventors; Promimic Ab, assignee. Synthetic nano-sized crystalline calcium phosphate and method of production. United States patent US 8206813 B2. 2012 Jun 26

23. Johansson P. Biomechanical evaluation and surface characterization of a nano modified surface on PEEK implants: a study in the rabbit tibia. Int J Nanomedicine. 2014;9:3903-3911.

24. Johansson P, Jimbo R, Kozai Y, et al. Nanosized hydroxyapatite coating on PEEK implants enhances early bone formation: a histological and three-dimensional investigation in rabbit bone. Materials. 2015;8(7):3815.

25. Pang KM, Lee JK, Seo YK, Kim SM, Kim MJ, Lee JH. Biologic properties of nano-hydroxyapatite: an in vivo study of calvarial defects, ectopic bone formation and bone implantation. Biomed Mater Eng. $2015 ; 25(1): 25-38$

26. Sandén B, Olerud C, Johansson C, Larsson S. Improved bone-screw interface with hydroxyapatite coating: an in vivo study of loaded pedicle screws in sheep. Spine. 2001;26(24):2673-2678

27. Gu Y, Zhang F, Lineaweaver WC, et al. In vivo study of hydroxyapatitecoated hat type cervical intervertebral fusion cage combined with IGF-I and TGF-beta1 in the goat model. J Spinal Disord Tech. Epub 2013 Nov 8 .

28. Suska F, Omar O, Emanuelsson L, et al. Enhancement of CRF-PEEK osseointegration by plasma-sprayed hydroxyapatite: a rabbit model. J Biomater Appl. 2014;29(2):234-242.

29. Nakahara I, Takao M, Goto T, Ohtsuki C, Hibino S, Sugano N. Interfacial shear strength of bioactive-coated carbon fiber reinforced polyetheretherketone after in vivo implantation. J Orthop Res. 2012; 30(10): 1618-1625.

30. Pelletier M, Cordaro N, Lau A, Walsh WR. PEEK versus Ti Interbody fusion devices: resultant fusion, bone apposition, initial and 26 week biomechanics. J Spinal Disord Tech. Epub 2012 Jul 13.

31. Sinclair SK, Konz GJ, Dawson JM, Epperson RT, Bloebaum RD. Host bone response to polyetheretherketone versus porous tantalum implants for cervical spinal fusion in a goat model. Spine. 2012;37(10): E571-E580.

\section{Publish your work in this journal}

The International Journal of Nanomedicine is an international, peerreviewed journal focusing on the application of nanotechnology in diagnostics, therapeutics, and drug delivery systems throughout he biomedical field. This journal is indexed on PubMed Central, MedLine, CAS, SciSearch $₫$, Current Contents $® /$ Clinical Medicine, Submit your manuscript here: http://www.dovepress.com/international-journal-of-nanomedicine-journ

\section{Dovepress}

Journal Citation Reports/Science Edition, EMBase, Scopus and the Elsevier Bibliographic databases. The manuscript management system is completely online and includes a very quick and fair peer-review system, which is all easy to use. Visit http://www.dovepress.com testimonials.php to read real quotes from published authors. 


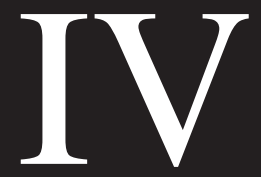



Biomechanical, histological and computed X-ray tomographic analyses of hydroxyapatite coated PEEK implants in an extended healing model in rabbit.

Pär Johansson ${ }^{1, *}$, Sargon Barkarmo ${ }^{2}$, Mohammed Hawthan ${ }^{3}$, Niccolò Peruzzi ${ }^{4}$, Per Kjellin ${ }^{5}$ and Ann Wennerberg ${ }^{2,}$

1 Department of Prosthodontics, Faculty of Odontology, Malmö University, Malmö, Sweden;

2 Department of Prosthodontics/Dental Materials Science, The Sahlgrenska Academy, Institute of Odontology, University of Gothenburg, Göteborg, Sweden;

3 Prosthodontic Department, Faculty of Dentistry, Umm AI-Qura University, Saudi Arabia;

4 Department of Clinical Sciences, Lund University, Lund, Sweden;

5 Promimic AB, AstraZeneca Bioventure Hub, Mölndal, Sweden.

* Author to whom correspondence should be addressed; E-Mail: par.johansson@mah.se; Tel.: +46-40-665 85 14; 
Abstract

A nanosized hydroxyapatite (HA) modification on polyetheretherketone (PEEK) using a novel spin coating technique was investigated in a rabbit model. Spin coating technique creates a 20-40 nm thick layer of HA particles with similar shape, size and crystallinity as human bone. Implants were designed with a perforating hole in the apical region to mimic a fusion chamber. The coating nano-structures were assessed using a scanning electron microscope. The in vivo response to HA-PEEK was compared to untreated PEEK with respect to removal torque, histomorphometry and computed microtomography. The HA-coated and pure PEEK implants were inserted in the tibia and femur bone according to simple randomization. The rabbits were sacrificed 20 weeks after implantation. Removal torque analysis showed significantly higher values for HA-PEEK. Qualitative histological evaluation revealed an intimate contact between PEEK and the bone at the threads and perforated hole. Histomorphometric assessment showed higher bone-implant and bone area values for HAPEEK but without statistical significance. The effect of the hydroxyapatite coating showed most prominent effect in the removal torque which may be correlated to an alteration in the bone quality around the HA-PEEK implants.

Keywords:

HA, polyether ether ketone, osseointegration, histology, hydroxyapatite, in vivo 
Introduction

Polyetheretherketone (PEEK) implants have been used in the orthopedic field since 1999 with the introduction of PEEK-OPTIMA ${ }^{\circledR}$ and has then gained new areas of application ${ }^{1-3}$. To mention a few applications, PEEK can today be found as spine cages for vertebral fusion, skull plates and friction components in articulating devices with successful and reliable outcomes $^{1,4,5}$.

PEEK has many positive qualities as a biomaterial compared to metal alloys. It is translucent to X-rays, which makes it possible to visualize the bone region around the implant site without artifacts. Unlike other thermoplastics, it can be sterilized using steam and gamma radiation ${ }^{1}$. Furthermore, PEEK has an elastic modulus in the region of trabecular bone and with the addition of carbon fibers it is possible to increase the strength significantly. With discontinuous fibers PEEK can even reach the mechanical properties of metal alloys ${ }^{1,6-9}$. The elastic modulus of PEEK reduces stress shielding and can in that way decrease bone resorption around the implants due to the mechanical similarity with bone $\mathrm{e}^{10,11}$.

PEEK is considered to be a relatively bioinert material and does not release ions, corrode or degrade in biological situations. However, some of these properties have been found in several studies to limit the cellular adhesion and the bone-implant contact when PEEK is compared with titanium ${ }^{12-14}$. Improving the bioactivity of PEEK is currently a major research topic and several methods and substances are under investigation. Several studies have shown an increased level of osseointegration using calcium phosphate coatings such as hydroxyapatite $(\mathrm{HA})$ on titanium ${ }^{15-17}$. The coating process for metal alloys usually involves high temperatures to produce a crystalline HA which HA incorporated in PEEK ${ }^{18,19}$, aerosol deposition $^{20}$ and cold spraying ${ }^{21,22}$. However, some of these techniques suffer from severe long term complications due to the thickness of the coating. The coating and impaired attachment to the surface have in retrieved implants shown fragments of delaminated coating. This may cause severe problems associated with inflammation and foreign body reactions ${ }^{23-25}$.

Recently published studies from our research group have been investigated the deposition of nano HA on PEEK implants using a spin-coat technique. The coating was applied using a liquid nano-sized HA solution and the excess was removed by spinning the implant under pressurized gas. After a short heat treatment $\left(325^{\circ} \mathrm{C}\right)$ the surface consists of an extremely thin (20-40 nm) HA coating with good adherence to the bulk material. The results have shown a significantly increased level of osseointegration after 3 and 12 weeks of healing ${ }^{26-28}$. However, the increased bone formation and removal torque were most prominent for HA- 
PEEK after 3 weeks compared to 12 weeks. One explanation could be that the early findings are influenced by the primary stability. It could as well be explained by a dissolution of the coating from 3 to 12 weeks.

Therefore in this study we are evaluating the level of osseointegration after a prolonged healing time of 20 weeks to investigate the delayed effect of the HA coating. The study protocol and design is similar to the previous reports to ensure a reliable comparison.

Materials and methods

Implant design and surface preparation

Polyether ether ketone implants (Invibio Ltd, Lancashire, UK) designed with a diameter of 3.5 $\mathrm{mm}$ and length of $4 \mathrm{~mm}$ were manufactured for this study (Figure 1). The study evaluated two kinds of apical designs for two different locations, one with a hole penetrating the apical part $(n=24)$ and one without $(n=48)$. The apical perforation was designed to enable an evaluation of the osteoconductive properties of the surface. Bone fusion into a volume is of clinical interest in several orthopedic applications, spinal fusion is one example. Overall, the study consisted of 72 implants where half of the implants $(n=36)$ were coated with $H A^{\text {nano }}$ Surface provided by Promimic AB, Göteborg, Sweden (test), whereas the remaining implants $(n=36)$ were left with their native surfaces (control).

The HA coating was applied on the surface as a liquid and then the implant were rotated at $2700 \mathrm{rpm}$ for 5 seconds to distribute the coating evenly. In the coating liquid, the HA crystals (20-50 nm long and 2-10 nm wide) were dispersed with water, surfactants and organic solvent. To remove the surfactants and stabilize the coating the implants were exposed to $325^{\circ} \mathrm{C}$ for 5 minutes under inert atmosphere. The final thickness of the coating was $20-40$ $\mathrm{nm}$. The coating procedure is in detail described in a patent description ${ }^{29}$.

\section{Scanning electron microscopy}

The morphology of the surface was evaluated using scanning electron microscopy (LEO Ultra 55 FEG; ZEISS, Oberkochen, Germany) at an accelerating voltage of 2-4 kV. The surface was sputtered with gold prior exposure to make it conductive with a JEOL JFC$1100 \mathrm{E}$ (JEOL Ltd, Tokyo, Japan) operating at $10 \mathrm{kV}$ for 90 seconds.

\section{Surgical procedure and removal torque}

Rabbits and surgical procedures were approved by the ethical committee for animal experiments at the Ecole Nationale Veterinaire D'Alfort, Maisons-Alfort, France and all experiments were performed following the institutional and national guidelines and 
regulations of the Board of Agriculture. A total of twelve mature male rabbits (New Zealand White rabbits) weighing between 3.3-4.2 kg were used in the study. General anesthesia was induced before the surgery using a dose of $250 \mu \mathrm{l} / \mathrm{kg}$ of medetomidine (Dormitor, Zoetis, France), $20 \mathrm{mg} / \mathrm{kg}$ of ketamine (Imalgene 1000, Merial, Sanofi, France) and $1 \mathrm{mg} / \mathrm{kg}$ of diazepam (Valium, Roche, Switzerland). The surgical area was shaved and disinfected with iodine solution. An incision was made medial of the planned implant locations through the skin, muscles and periosteum on tibia and femur. The implant site was prepared using rotating burs to a final diameter of $3.2 \mathrm{~mm}$. Tapping and implant insertion was performed by hand. Each rabbit received three coated and three uncoated implants in the hind legs; one implant in the femur with apical perforation, one implant in distal tibia and one in the proximal tibia (Figure 2). The perforated implants were selected for the femur because of two reasons; the entire length of the implants will be surrounded by bone and the trabecular bone mimics the clinical situation of a spine implant. The femur bone consists mostly of trabecular bone and the tibia has a cortical layer outside the bone marrow. These different qualities are intended to equal the two most common bone types in dental and orthopedic applications. The locations of test and control implants were determined by simple randomization ${ }^{30}$. The epithelium and fascia were closed separately with bioresorbable sutures (4-0 Vicryl, Ethicon, Auneau, France). Post-operative analgesic was administrated by injection of buprenorphine (Buprecare, Animalcare, UK) and meloxicam (Metacam; Boehringer Ingelheim Vetmedica, Inc., St Joseph, MO, USA). Additional analgesic (fentanyl, Duragesic, Janssen Pharmaceutica, Beerse, Belgium) was applied to the skin for three days and also antibiotics (enrofloxacine, Baytril, Bayer Animal Health, Germany) for five days through the drinking water $(200 \mathrm{ml} / \mathrm{l})$. The animals were euthanized 20 weeks after the surgery with intravenous overdose of sodium pentobarbital (Euthasol, Virbac, Fort Worth, TX, USA).

The tibia and femur were dissected and removed immediately after the euthanasia and soft tissue was cleared from the implant head. The implant at the distal site of tibia was coupled to a digital torque meter (Tohnichi, Tokyo, Japan) and the maximal removal torque (RTQ) was recorded in $\mathrm{Ncm}(n=24)$.

\section{Computed tomography}

Twelve sections of samples with apical perforation, six test and six control, were imaged with $x$-ray computed microtomography $(\mu \mathrm{CT})$. The measurements were performed using a ZEISS Xradia Versa $520 \mathrm{X}$-ray Microscope at the 4D Imaging lab in Lund University, with the source set at $80 \mathrm{kV}$ and $7 \mathrm{~W}$ and with an acquisition time of approximately $2 \mathrm{~h}$ (3201 projections) per sample. Each tomography had a voxel size of $5 \times 5 \times 5 \mu \mathrm{m}^{3}$, with a field of view of roughly 1 $\mathrm{cm}$ in each direction. 
The image analysis was performed with the Amira software package (FEI Visualization Sciences Group, Bordeaux, France). The high similarity between the absorption coefficients of PEEK and of the light-cured resin, in which the samples were embedded, prevented the use of semi-automatic segmentation techniques. For this reason, the volume of the apical perforations was segmented manually. The volume of the newly formed bone inside the perforations could then be segmented using threshold based methods (Figure 3). Two test samples and one control sample had to be discarded from the subsequent analysis from the $\mu C T$ scan since they appeared to have been damaged during the sectioning procedure.

The bone volume fraction ( \%) inside the apical perforation was calculated from the entrance until a depth of $400 \mu \mathrm{m}$ into the hole. In order to have an indication about the bone growth rate as a function of depth, the concept of cumulative BV/TV (Bone volume/Total volume) was also introduced. It consists in recalculating the BV/TV at each subsequent step of depth ( $5 \mu \mathrm{m}$ step-size), adding progressively to the volume of interest (VOI) the corresponding new slice of volume. At zero depth it coincides with the bone area fraction (BA, \%) at the entrance of the perforation, at every other depth it corresponds to the bone volume fraction in a VOI which goes from the entrance until that particular depth.

\section{Histomorphometric analysis}

After the RTQ measurements, the implant placed in the femur bone and the remaining one in the tibia were removed en bloc with surrounding bone $(n=48)$. The blocks were fixated in formalin and dehydrated in increasing concentrations of ethanol (Solveco $A B$ ) up to $99.9 \%$ and then embedded in light-cured resin (Technovit 7200 VLC; Heraeus Kultzer, Wehrheim, Germany). The samples were cut along the implant axis using a cutting-grinding system (Exakt; Apparatebau, Norderstedt, Germany). The sections were ground to a final thickness of $\sim 40 \mu \mathrm{m}$ and thereafter stained with toluidine blue. The bone-implant contact (BIC, \%) and the bone area (BA, \%) in all the thread were measured on both sides of the entire length of the implants. Since the implant in the tibia penetrates the bone marrow, the calculation of $\mathrm{BIC}$ and BA was also limited to the top three threads in order to obtain more consistent data.

The sections $(n=48)$ were analyzed using a light microscope (Eclipse ME600, Nikon Corporation, Tokyo, Japan), and histological images were captured by a digital camera (DSRi2, Nikon Corporation, Tokyo, Japan) coupled with the microscope. The images were imported and analyzed in an image analysis software (Image J Version 1.43r; National Institutes of Health, Bethesda, MD, USA) by a blind to the treatment protocol examiner.

\section{Statistics}

All quantitative data are presented as mean \pm standard deviations. The differences between test and control of the removal torque and histomorphometric values were analyzed with a nonparametric Wilcoxon signed rank test. For both methods a level of significance was set at 
$P<0.05$. Analysis was performed using SPSS software (Version 22, IBM, Armonk, NY, USA).

The results from the micro-CT were averaged between the samples in each of the two groups (test and control); the final results are presented as mean and standard deviation of the mean. A Welch's t-test was also used to compare the two groups among them, with a level of significance set at $P<0.05$. The data analysis was performed in MATLAB environment.

Results

SEM

The coating process was found to be effective as the minerals cover the surface and were evenly distributed on the surface. The underlying milling texture is concealed by the coating and all the samples are equally covered by the coating. Figure 4 shows an uncoated PEEK surface (left) and an HA-coated surface (right).

\section{Removal torque}

All the animals were in good health at the time of sacrifice without any signs of inflammation or infection. As demonstrated in figure 5 the HA modified PEEK implants from the distal tibia showed a significantly higher removal torque compared to native PEEK implants $(P=0.028)$. The mean values for test and control were $6.42 \mathrm{Ncm}( \pm 3.32)$ and $4.04 \mathrm{Ncm}( \pm 1.39)$, respectively.

\section{Histomorphometric analysis}

After 20 weeks of healing a complete and mature bone formation was observed immediately against the surface of the implants in both groups. In the qualitative evaluation woven bone and lamellar bone were found in contact with the implant surface inside the threads and at the external surfaces (Figure 6A-D). The thickness of the tibia differs between different rabbits and implant site, resulting in variations in coronal threads in contact with cortical bone. The bone inside the apical perforation extends from the entrance into the center of the hole with close contact to the implant surface (Figure 7A-B). In some sections where the hole was viewed from a perpendicular angulation, the trabecular bone shape was visible and bone covers most of the inner surface.

The quantitative evaluation after 20 weeks of healing in rabbit femur revealed higher BA and $\mathrm{BIC}$ values for test implants but without statistical significance $(B A: P=0.213, B I C: P=$ 0.441). In the tibia there was no significant difference of $B A$ and $B I C$ values between the groups when measuring the whole part of the implant. Bone area and BIC in the top three 
threads of tibia after 20 weeks were higher for the test group with values of $58,2 \%$ (BA) and $21.8 \%$ (BIC) compared to values of $53.1 \%(B A)$ and $17.9 \%$ for the control (Table 1).

\section{Computed tomography}

The resulting mean value of the BV/TV in a $400 \mu m$-deep VOI inside the apical perforation was $27 \% \pm 4 \%$ for the test samples and $21 \% \pm 8 \%$ for the control ones. The comparison between the two groups returns a $p$-value of $P=0.56$, indicating that the low number of samples does not allow to achieve statistically meaningful conclusions. The mean cumulative BV/TV (Figure 8) does not show significant differences in the behaviour of the two groups. The lower variance at higher depths seems to indicate that the reduction rate of the BV/TV is faster for samples with high starting values of BV/TV.

\section{Discussion}

In our previous reports we have described and investigated the effect of a nano-sized HA coating on PEEK implants after 3 and 12 weeks of healing in rabbit bone $e^{26-28}$. The results were convincing and revealed a significantly higher degree of osseointegration for coated implants compared to neat PEEK. Although, a trend of decreased difference in BIC, BA and RTQ was revealed between the groups at the later time point. These results motivated the decision of conducting a study with an extended healing time in order to reveal a comprehensive picture of the healing and bone formation.

Osseointegration, a direct contact between new bone and implant is an event that is dependent on several factors ${ }^{31}$. Surface topography is one of the dependent factors to establish osseointegration according to Wennerberg and Albrektsson ${ }^{32}$. In addition, bioactive surface modifications have been found to enhance the osseointegration during the early stages of healing ${ }^{33}$. The coating procedure used in this study altered the surface roughness at the nanometer level but also improved the bioactive appearance ${ }^{34}$.

One way to measure the level of osseointegration in experimental models is RTQ. Removal torque measures the total force required to interrupt the interface between bone and implant. In this study we found significantly higher removal torque values for the HA coated PEEK implants. In addition, HA-coated PEEK implants have in a previous study demonstrated significantly higher removal torque values at 3 and 12 weeks of healing. Therefore, the results indicate that HA-coated implants have a higher retention in tibia bone regardless of healing time. The underlying biomechanics at the disruption of bone-implant contact is a complex mechanism. Stenlund et $a l .^{35}$ established an experimental model to investigate the mechanism and factors affecting implant retention. They discovered that the surface roughness and properties of the surrounding bone affects the implant retention. The surface roughness of the implants can be standardized but the anatomical variations of the implant bed cannot be eliminated. In addition, the implant location may differ between the rabbits where some implants may be placed in thicker cortical bone than others. Palmquist et al. ${ }^{36}$ 
and Branemark et al. ${ }^{37}$ compared the RTQ fracture patterns at the interface of bone-implant. Conclusions were made that nano-structured surfaces promote long term bonding and interface strength. This study further reveals that the nature of surrounding material (bone) affects the outcome using identical implants. It can be hypothesized that HA-coating not only improves osseointegration but also increases the maturation of the surrounding bone resulting in higher RTQ values.

Compared with metallic implants, PEEK is porous and sensitive to abrasive force, had an impact in the histological sample sectioning.. At the optimal thickness of the sections, the PEEK material almost disappeared in the grinding process. Therefore the sections were made thicker but as a consequence the surrounding bone were in some samples difficult to measure. Histomorphometric analysis have in our previous studies showed significantly increased BIC, BA inside thread and BA inside hole after 3 and 12 weeks of healing. This trend was changed in this study of 20 weeks healing time where no statistical differences could be determined in any of the measuring aspects.

A study by Nakahara et al. ${ }^{36}$ compared the interfacial shear strength between HA-coated and uncoated PEEK implants after 2 different healing times. The mechanical test revealed higher retention for HA-coated PEEK after 6 and 12 weeks of healing. This resembles with the findings from the present study except that the healing times varies.

Søballe et al. ${ }^{37}$ revealed an osteoconductive effect of HA coating on titanium implants at a distance of 400 microns from the surface in a gap healing model. This positive gradient was not found in the group without HA-coating. This demonstrates an effect of HA not only at the surface but also in the surrounding tissue. The results from Søballe and co-workers may explain why BIC in this study remains similar but the RTQ are different at an extended healing time. The aforementioned study was not performed on PEEK, but the HA coating would almost certainly behave similar regardless on the bulk material.

To minimize the drawbacks of thick coating and risk of delamination the research has been conducted and directed towards thinner coating. This spin-coated implants in this study has a coating of approximately $20-40 \mathrm{~nm}$. The coating stability was evaluated in a previous study from this research group and revealed signs of coating detachment on the thread edges ${ }^{27}$. However, in the thread valleys and cracks the coating was well-preserved.

The histomorphometric results from this and our previous studies indicate that the effect of the HA-coating on bone formation was most significant at 3 weeks and 12 weeks. However, after20 weeks of healing the results showed no significant difference in terms of BIC and BA. To further address this outline the focus goes toward the thickness and dissolution of the HAcoating. Nano-thickness and plasma-sprayed HA-coated implants were compared by Quaranta and co-workers ${ }^{38}$ in a rabbit model. Both thicknesses were considered to show osteoconductive properties, but the thicker plasma-sprayed coating was favored in the early 
bone response with respect to BIC and BA. Studies have demonstrated similar histological behaviors when comparing coating thickness of 50 and $200 \mu \mathrm{m}^{39}$ and in the range $1-15 \mu \mathrm{m}^{40}$ and all positively affected the osseointegration compared to the uncoated ones. But no significant effect was found in between the groups of different thicknesses. Logically, amorphous coatings would resorb faster than crystalline which Wolke et al. ${ }^{41}$ validates when evaluating an ultrathin amorphous coating $(0.1 \mu \mathrm{m})$ which was resorbed after 1 week postimplantation. According to Darimont et $a{ }^{42}$ an early bone formation at the implant surface may prevent the coating from being dissolved.

The $\mu \mathrm{CT}$ results are consistent with the histomorphometric results, showing no significant difference between the two groups. A considerable limit that had to be faced when analysing the samples with $\mu \mathrm{CT}$ was the poor absorption contrast between PEEK and the light-cured resin in which the samples were embedded. The use of other embedding materials, with a different absorption coefficient, might improve future $\mu C T$ scans in multiple ways: it would allow to identify the implant and zoom on it when setting up the scan, further improving the voxel size, and it could enable the use of semi-automatic segmentation techniques, more reliable than manual ones. A better segmentation would allow to measure other relevant properties of the samples, such as BIC.

In this work the HA-coating is crystalline and should therefore remain longer on the surface compared to an amorphous coating, but when finally dissolved, the succeeding osseointegration may be dependent on the substrate properties. We hypothesize that the hydroxyapatite possess an osteoconductive effect at the early time points but after an extended healing period the coating was consumed or dissolved, resulting in comparable BIC-values between the groups. The higher RTQ values for HA-coated PEEK cannot be related to the histomorphometric results, but may be explained by an alteration in the quality of the surrounding bone. However, the computed tomographic results failed to reveal any significant difference in bone density. Therefore, the underlying reasons for the improved RTQ cannot be explain within the limitations of this study.In orthopedics and spinal surgery, enhanced early bone formation inside the implant perforation and the implant surface may improve the treatment outcomes and minimize the risk of infections. The long-term bone formation may not be as critical in spine surgery, on condition that the implant remain stable without disruptive micro-motions. In dental implants on the other hand, an increased retention in bone may have a more major role than bone formation with regards to withstanding continuous masticatory loading. 


\section{CONCLUSIONS}

In this study of an experimental in vivo project evaluating crystalline nanosized coating on PEEK implant we may conclude that the positive effects were expressed differently over the healing times. From an enhanced new bone formation at the implant surface at 3 and 12 weeks to matured bone forming an increased interfacial shear strength at 20 weeks of healing.

This results contributes to the field and may provide knowledge to improving the coating properties to improve the bioactivity of PEEK implants.

\section{ACKNOWLEDGEMENTS}

This study was supported by grants achieved from the Swedish Research Council and The King Gustav V and Queen Victoria foundation, the Swedish order of Freemasons.

1. Kurtz SM, Devine JN. PEEK biomaterials in trauma, orthopedic, and spinal implants. Biomaterials 2007;28(32):4845-69.

2. Poulsson AH, Eglin D, Zeiter S, Camenisch K, Sprecher C, Agarwal Y, Nehrbass D, Wilson J, Richards RG. Osseointegration of machined, injection moulded and oxygen plasma modified PEEK implants in a sheep model. Biomaterials 2014;35(12):371728.

3. Rivard $\mathrm{CH}$, Rhalmi S, Coillard C. In vivo biocompatibility testing of peek polymer for a spinal implant system: a study in rabbits. J Biomed Mater Res 2002;62(4):488-98.

4. Kulkarni AG, Hee HT, Wong HK. Solis cage (PEEK) for anterior cervical fusion: preliminary radiological results with emphasis on fusion and subsidence. Spine J 2007;7(2):205-9.

5. Li CS, Vannabouathong C, Sprague S, Bhandari M. The Use of Carbon-FiberReinforced (CFR) PEEK Material in Orthopedic Implants: A Systematic Review. Clin Med Insights Arthritis Musculoskelet Disord 2015;8:33-45.

6. Kurtz SM. PEEK biomaterials handbook: Elsevier; 2012.

7. Nieminen T, Kallela I, Wuolijoki E, Kainulainen H, Hiidenheimo I, Rantala I. Amorphous and crystalline polyetheretherketone: Mechanical properties and tissue reactions during a 3-year follow-up. J Biomed Mater Res A 2008;84(2):377-83.

8. Ponnappan RK, Serhan H, Zarda B, Patel R, Albert T, Vaccaro AR. Biomechanical evaluation and comparison of polyetheretherketone rod system to traditional titanium rod fixation. Spine J 2009;9(3):263-7.

9. Chou WK, Chien A, Wang JL. Biomechanical analysis between PEEK and titanium screw-rods spinal construct subjected to fatigue loading. J Spinal Disord Tech 2015;28(3):E121-5.

10. Steinberg EL, Rath E, Shlaifer A, Chechik O, Maman E, Salai M. Carbon fiber reinforced PEEK Optima--a composite material biomechanical properties and wear/debris characteristics of CF-PEEK composites for orthopedic trauma implants. J Mech Behav Biomed Mater 2013;17:221-8.

11. McNamara LM, Van der Linden JC, Weinans H, Prendergast PJ. Stress-concentrating effect of resorption lacunae in trabecular bone. J Biomech 2006;39(4):734-41. 
12. Sagomonyants KB, Jarman-Smith ML, Devine JN, Aronow MS, Gronowicz GA. The in vitro response of human osteoblasts to polyetheretherketone (PEEK) substrates compared to commercially pure titanium. Biomaterials 2008;29(11):1563-72.

13. Lu T, Qian S, Meng F, Ning C, Liu X. Enhanced osteogenic activity of poly ether ether ketone using calcium plasma immersion ion implantation. Colloids Surf B Biointerfaces 2016;142:192-8.

14. Toth JM, Wang M, Estes BT, Scifert JL, Seim HB, 3rd, Turner AS. Polyetheretherketone as a biomaterial for spinal applications. Biomaterials 2006;27(3):324-34.

15. Surmenev RA, Surmeneva MA, Ivanova AA. Significance of calcium phosphate coatings for the enhancement of new bone osteogenesis--a review. Acta Biomater 2014;10(2):557-79.

16. Zhang BG, Myers DE, Wallace GG, Brandt M, Choong PF. Bioactive coatings for orthopaedic implants-recent trends in development of implant coatings. Int J Mol Sci 2014;15(7):11878-921.

17. Bai X, Sandukas S, Appleford MR, Ong JL, Rabiei A. Deposition and investigation of functionally graded calcium phosphate coatings on titanium. Acta Biomater 2009;5(9):3563-72.

18. Ma R, Yu Z, Tang S, Pan Y, Wei J, Tang T. Osseointegration of nanohydroxyapatiteor nano-calcium silicate-incorporated polyetheretherketone bioactive composites in vivo. Int J Nanomedicine 2016;11:6023-6033.

19. Ma R, Li Q, Wang L, Zhang X, Fang L, Luo Z, Xue B, Ma L. Mechanical properties and in vivo study of modified-hydroxyapatite/polyetheretherketone biocomposites. Mater Sci Eng C Mater Biol Appl 2017;73:429-439.

20. Rao PJ, Pelletier MH, Walsh WR, Mobbs RJ. Spine interbody implants: material selection and modification, functionalization and bioactivation of surfaces to improve osseointegration. Orthop Surg 2014;6(2):81-9.

21. Lee JH, Jang HL, Lee KM, Baek HR, Jin K, Noh JH. Cold-spray coating of hydroxyapatite on a three-dimensional polyetheretherketone implant and its biocompatibility evaluated by in vitro and in vivo minipig model. J Biomed Mater Res B Appl Biomater 2017;105(3):647-657.

22. Gardon M, Melero H, Garcia-Giralt N, Dosta S, Cano IG, Guilemany JM. Enhancing the bioactivity of polymeric implants by means of cold gas spray coatings. J Biomed Mater Res B Appl Biomater 2014;102(7):1537-43.

23. Cheang $\mathrm{P}$, Khor KA. Addressing processing problems associated with plasma spraying of hydroxyapatite coatings. Biomaterials 1996;17(5):537-44.

24. Rokkum M, Reigstad A, Johansson CB. HA particles can be released from well-fixed HA-coated stems: histopathology of biopsies from 20 hips 2-8 years after implantation. Acta Orthop Scand 2002;73(3):298-306.

25. Albrektsson T. Hydroxyapatite-coated implants: a case against their use. J Oral Maxillofac Surg 1998;56(11):1312-26.

26. Johansson P. Biomechanical evaluation and surface characterization of a nano modified surface on PEEK implants: A study in the rabbit tibia. Int $\mathbf{J}$ Nanomedicine 2014.

27. Johansson P, Jimbo R, Kozai Y, Sakurai T, Kjellin P, Currie F, Wennerberg A. Nanosized Hydroxyapatite Coating on PEEK Implants Enhances Early Bone Formation: A Histological and Three-Dimensional Investigation in Rabbit Bone. Materials 2015;8(7):3815. 
28. Johansson P, Jimbo R, Naito Y, Kjellin P, Currie F, Wennerberg A. Polyether ether ketone implants achieve increased bone fusion when coated with nano-sized hydroxyapatite: a histomorphometric study in rabbit bone. Int $\mathrm{J}$ Nanomedicine 2016;11:1435-42.

29. Kjellin P, Andersson M. Synthetic nano-sized crystalline calcium phosphate and method of production. Google Patents; 2012.

30. Suresh K. An overview of randomization techniques: An unbiased assessment of outcome in clinical research. J Hum Reprod Sci 2011;4(1):8-11.

31. Albrektsson T, Branemark PI, Hansson HA, Lindstrom J. Osseointegrated titanium implants. Requirements for ensuring a long-lasting, direct bone-to-implant anchorage in man. Acta Orthop Scand 1981;52(2):155-70.

32. Wennerberg A, Albrektsson T. Effects of titanium surface topography on bone integration: a systematic review. Clin Oral Implants Res 2009;20 Suppl 4:172-84.

33. Meng HW, Chien EY, Chien HH. Dental implant bioactive surface modifications and their effects on osseointegration: a review. Biomark Res 2016;4:24.

34. Johansson P, Jimbo R, Kjellin P, Currie F, Chrcanovic BR, Wennerberg A. Biomechanical evaluation and surface characterization of a nano-modified surface on PEEK implants: a study in the rabbit tibia. Int J Nanomedicine 2014;9:3903-11.

35. Stenlund P, Murase K, Stalhandske C, Lausmaa J, Palmquist A. Understanding mechanisms and factors related to implant fixation; a model study of removal torque. $\mathrm{J}$ Mech Behav Biomed Mater 2014;34:83-92.

36. Nakahara I, Takao M, Goto T, Ohtsuki C, Hibino S, Sugano N. Interfacial shear strength of bioactive-coated carbon fiber reinforced polyetheretherketone after in vivo implantation. J Orthop Res 2012;30(10):1618-25.

37. Soballe K. Hydroxyapatite ceramic coating for bone implant fixation. Mechanical and histological studies in dogs. Acta Orthop Scand Suppl 1993;255:1-58.

38. Quaranta A, Iezzi G, Scarano A, Coelho PG, Vozza I, Marincola M, Piattelli A. A histomorphometric study of nanothickness and plasma-sprayed calcium-phosphorouscoated implant surfaces in rabbit bone. J Periodontol 2010;81(4):556-61.

39. Yang CY, Wang BC, Lee TM, Chang E, Chang GL. Intramedullary implant of plasma-sprayed hydroxyapatite coating: an interface study. J Biomed Mater Res 1997;36(1):39-48.

40. Lee J-H, Kim S-G, Lim S-C. Histomorphometric study of bone reactions with different hydroxyapatite coating thickness on dental implants in dogs. Thin Solid Films 2011;519(15):4618-4622.

41. Wolke JG, van der Waerden JP, Schaeken HG, Jansen JA. In vivo dissolution behavior of various RF magnetron-sputtered $\mathrm{Ca}-\mathrm{P}$ coatings on roughened titanium implants. Biomaterials 2003;24(15):2623-9.

42. Darimont GL, Cloots R, Heinen E, Seidel L, Legrand R. In vivo behaviour of hydroxyapatite coatings on titanium implants: a quantitative study in the rabbit. Biomaterials 2002;23(12):2569-75. 
FIGURE LEGENDS

Figure 1: Schematic image of PEEK implant

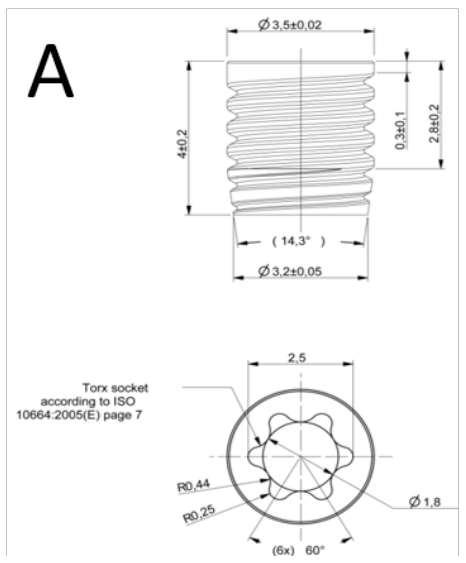

B

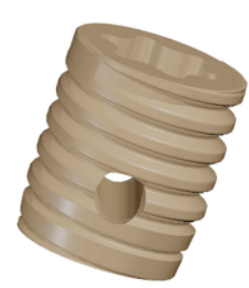




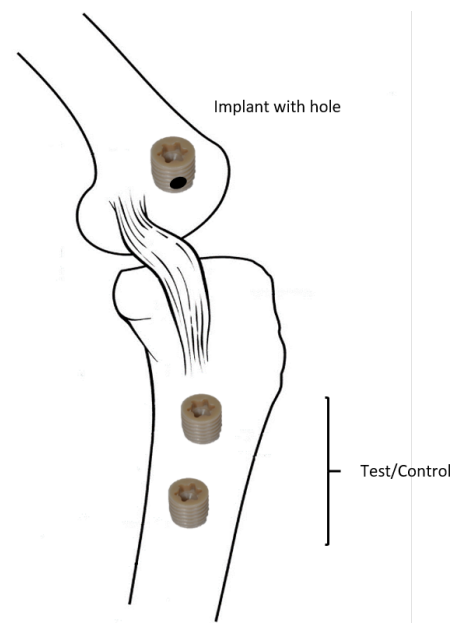

Figure 2: Graphical description of the implant positioning in femur and tibia. The implant with a perforated hole is placed in the femur bone. The location of test and control samples is randomized
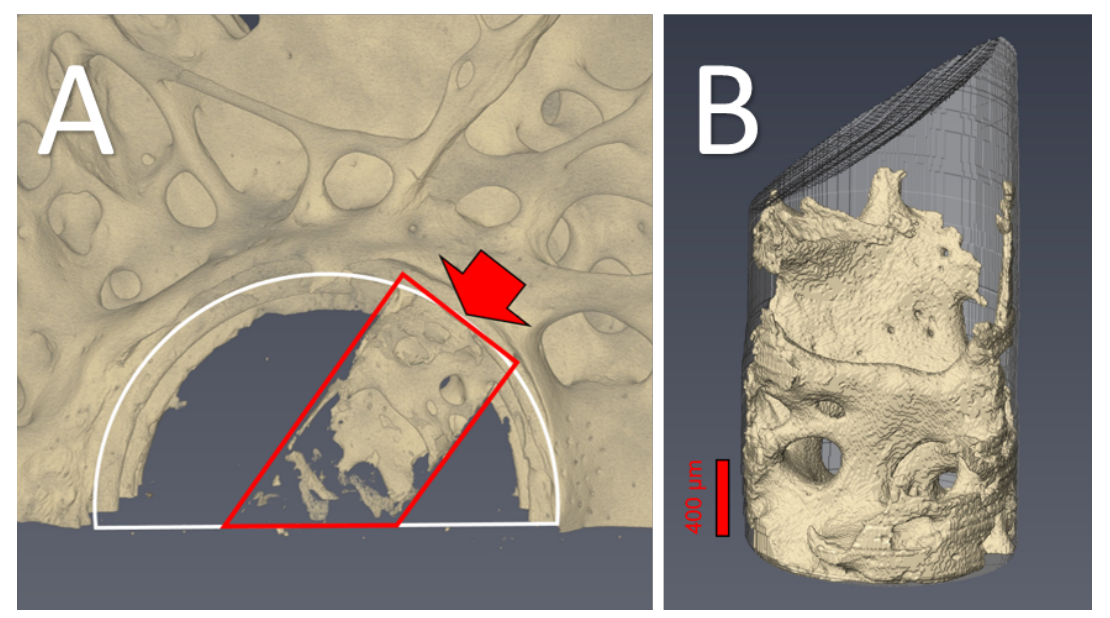

Figure 3A-B: $\mathbf{A}-3 D$ rendering of a $\mu C T$ scan, viewpoint along the rotation axis of the implant. The section of the PEEK implant cannot be distinguished from the background, and appears as a hollow semi-cylindrical area (white outline) in the bone structure; the red outline delimits the apical perforation, of which the red arrow marks the entrance. B - Segmentation of the apical perforation (in transparent light grey) and of the bone inside it. The red bar shows the scale $(400 \mu \mathrm{m})$. 


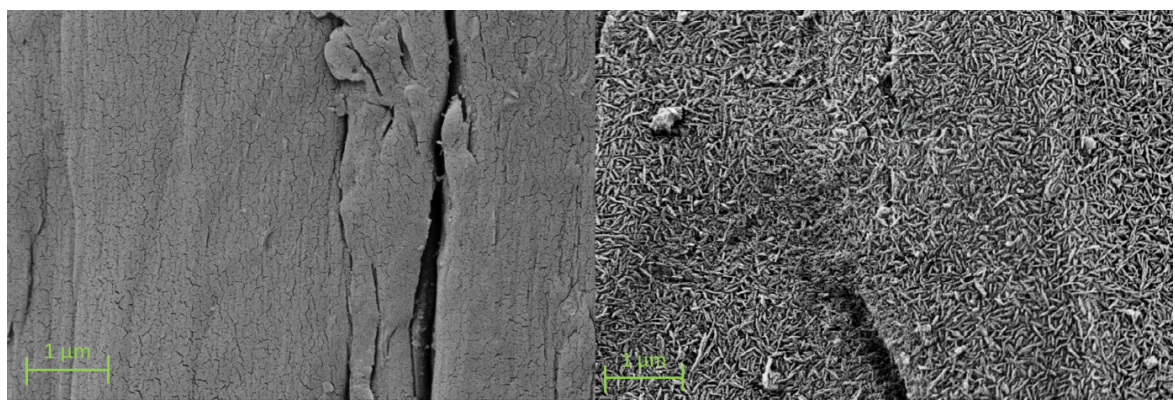

Figure 4: SEM image of the surface topography at the implant thread portion. Left: Pure PEEK. Right: Hydroxyapatite coated PEEK. Bar $1 \mu \mathrm{m}$

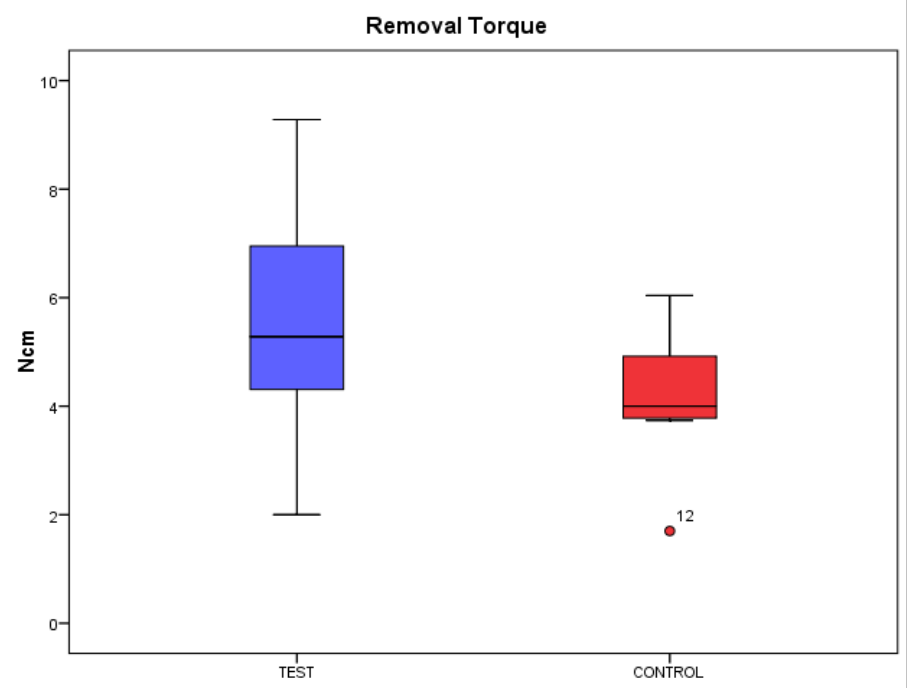

Figure 5: Box plot representing the RTQ values for test and control after 20 weeks of healing. Standard deviation displayed with error bars. ${ }^{*} P<0.05$. 


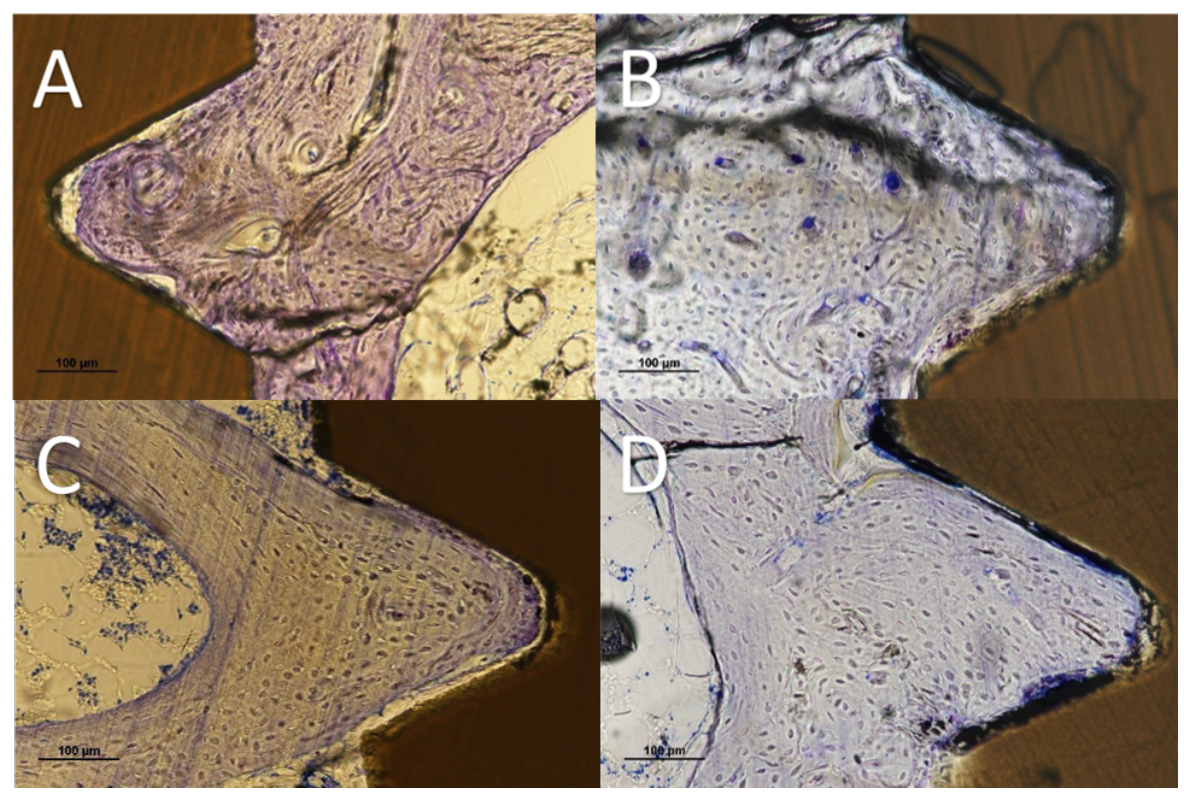

Figure 6A-D: Representative histological micrographs. A: Tibia, HA-PEEK. B: Tibia, PEEK. C: Femur, HA-PEEK. D: Femur, PEEK. Bar 100um
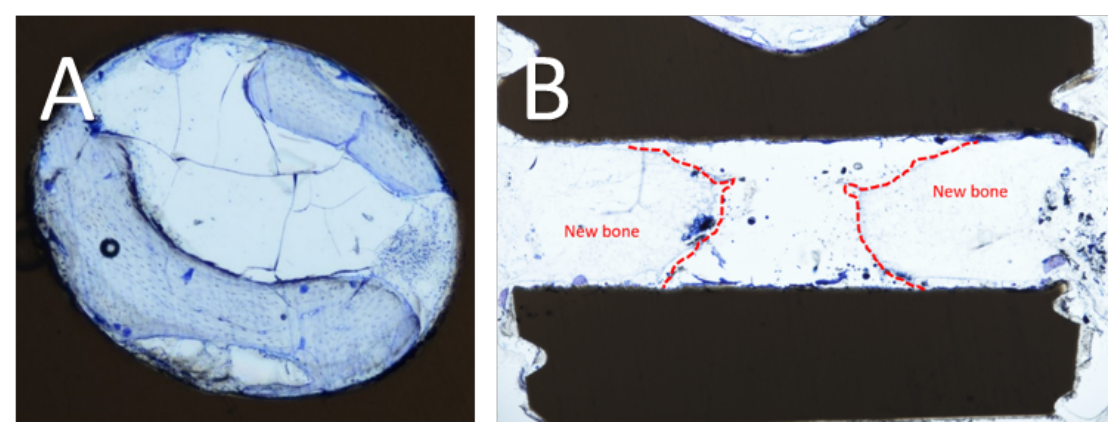

Figure 7A-B: Histological section showing the apical perforation in parallel and perpendicular view of two different HA coated implants. 


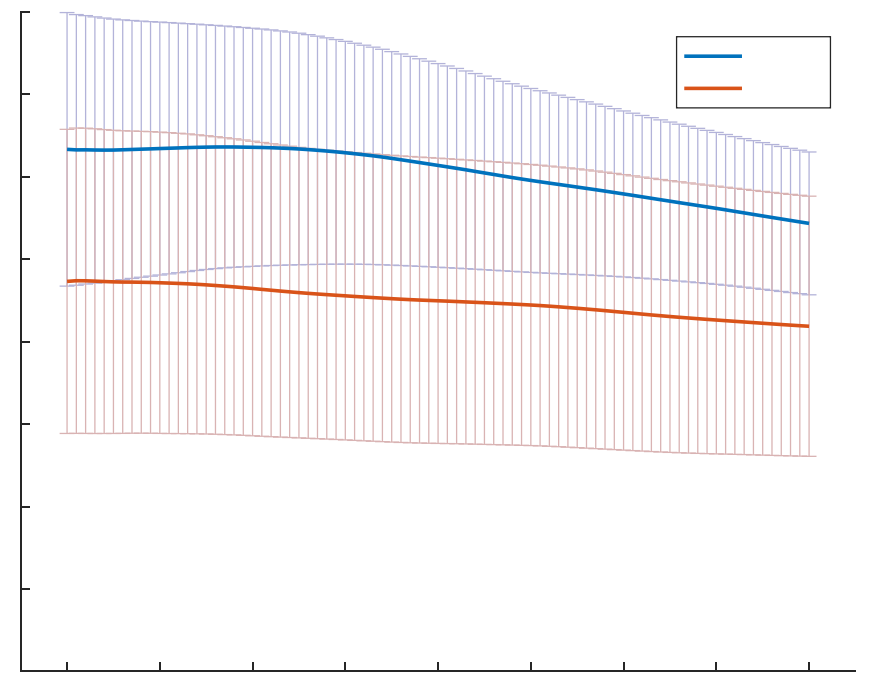

$\mu$

Figure 8: The mean cumulative BV/TV as a function of the depth inside the apical perforation, for the test and the control samples. The standard deviation of the mean is displayed as error bars.

\begin{tabular}{llcccc} 
Location & Group & BIC & Top3-BIC & BA & Top3-BA \\
Tibia & Test & .1283 & .2188 & .3821 & .5826 \\
& & & & & .5313 \\
& Control & .1546 & .1796 & .173 & .066 \\
Femur & P-value & .086 & .110 & .5732 & .5143 \\
\hline & Test & .1947 & & .213 \\
\hline
\end{tabular}

Table 1 - Histomorphometric results: Mean values of the percentage of bone implant contact (BIC), Bone area (BA) and values exclusively from the top 3 threads. Standard deviation is given within parenthesis. Results were analyzed with a nonparametric Wilcoxon signed rank test with significance level at $p \leq 0.05$. 

ISBN 978-9I-7 IO4-8OI-I (print)

ISBN 978-9I-7 IO4-8O2-8 (pdf)

MALMÖ UNIVERSITY 20506 MALMÖ, SWEDEN WWW.MAH.SE 